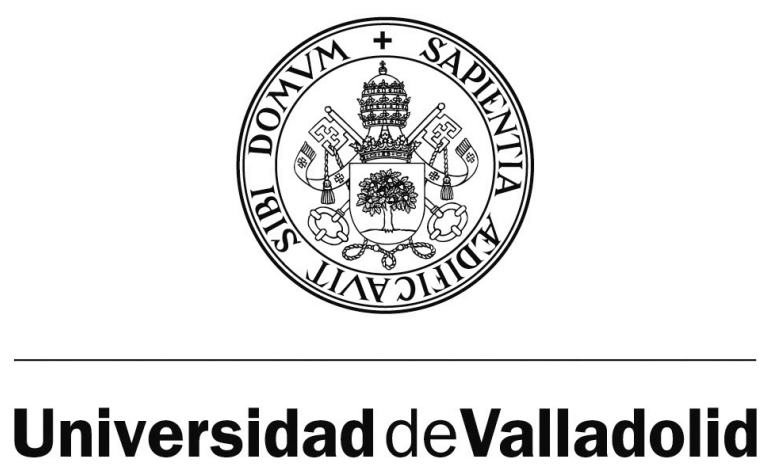

FACULTAD DE MEDICINA

DEPARTAMENTO DE PEDIATRÍA, INMUNOLOGÍA, OBSTETRICIA Y GINECOLOGÍA, NUTRICIÓN Y BROMATOLOGÍA, PSIQUIATRÍA E HISTORIA DE LA CIENCIA

TESIS DOCTORAL:

\title{
ANÁLISIS DEL TIEMPO QUIRÚRGICO COMO FACTOR DE RIESGO EN CIRUGÍA NEONATAL
}

Presentada por Agustín del Cañizo López para optar al grado de doctor por la Universidad de Valladolid

Dirigida por:

Dr. José Manuel Marugán Miguelsanz (Director)

Dr. José Herreros Rodríguez (Codirector) 



\section{DEDICATORIA:}

Al Dr. Cerdá sin cuya amistad y apoyo nunca hubiera logrado convertirme en cirujano pediátrico y sin cuya ayuda no podría haber llevado a cabo esta tesis. A Aida e Irene, por su paciencia y cariño durante estos estresantes meses . A mi padre, que siempre ha deseado que sea doctor. 


\section{AGRADECIMIENTOS:}

Deseo expresar mi más sincero agradecimiento al Dr. Agustín Mayo por su inestimable ayuda con el análisis estadístico de los datos. También a mis compañeros del Gregorio Marañón por la colaboración en la recogida de datos. Al Dr. José Herreros por sus útiles consejos y correcciones y al Dr. Marugán quien aceptó dirigir la tesis pese a su gran número de obligaciones.

A mis compañeros del Clínico de Valladolid por facilitarme el trabajo.

Finalmente al Dr. Juan Luis Gómez por sus rapidísimas gestiones en la Universidad de Salamanca y por sus consejos y paciencia. 


\section{RESUMEN:}

\section{Introducción:}

Por sus características especiales el recién nacido es especialmente sensible a la hipotermia, al sangrado y la infección.

Por otro lado el tiempo quirúrgico es un factor pronóstico en otros pacientes especialmente sensibles a la hipotermia y el sangrado e influye en el riesgo de infección postoperatoria.

\section{Objetivo:}

Determinar si el tiempo quirúrgico es un factor de riesgo en el neonato intervenido.

\section{Material y métodos:}

Realizamos un estudio retrospectivo del los neonatos intervenidos en el Hospital Gregorio Marañón entre 2008 y 2011. Analizando la relación entre tiempo quirúrgico y tiempo total de quirófano con mortalidad, complicaciones, reintervenciones, tiempo de ingreso total y el tiempo de ingreso en Unidad de Cuidados Intensivos Neonatales (UCIN).

\section{Resultados:}

Existe una relación estadísticamente significativa entre tiempo quirúrgico y tiempo total de quirófano con las complicaciones, reintervenciones y tiempo de ingreso en UCIN .

No hallamos relación estadísticamente significativa con la mortalidad y el tiempo de ingreso en la totalidad de pacientes pero sí en grupos concretos como los pacientes sometidos a laparotomías y toracotomías o los diagnosticados de Ductus Arterioso Persistente. 
Los pacientes con Enterocolitis Necrotizante y aquellos intervenidos en la UCIN tuvieron menos relaciones significativas con el tiempo quirúrgico que el resto.

\section{Conclusiones:}

Tiempo quirúrgico y tiempo total de quirófano son factores de riesgo en cirugía neonatal.

Influyen en la frecuencia de complicaciones y reintervenciones y el tiempo de ingreso en UCIN en la totalidad de los pacientes y en la mortalidad y tiempo total de ingreso de grupos concretos. 


\section{ÍNDICE:}

INTRODUCCIÓN

- 1. CONCEPTO Y JUSTIFICACIÓN DE LA CIRUGÍA PEDIÁTRICA COMO ESPECIALIDAD

- 2. EL NEONATO COMO PACIENTE ..............................................................17

- 3. FACTORES PRONÓSTICO EN CIRUGÍA NEONATAL..................................22

- 4. EL TIEMPO QUIRÚRGICO COMO FACTOR DE RIESGO ...............................33

- 5. TIEMPO QUIRÚRGICO Y CIRUGÍA NEONATAL...........................................39

- 6. OTROS FACTORES RELACIONADOS CON EL TIEMPO QUIRÚRGICO Y SU INFLUENCIA EN EL PRONÓSTICO..............................................................43

OBJETIVOS

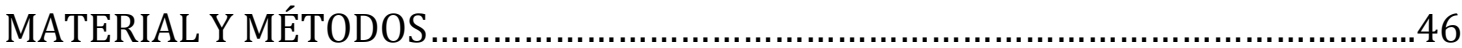

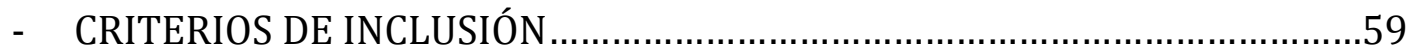

- CRITERIOS DE EXCLUSIÓN...............................................................................

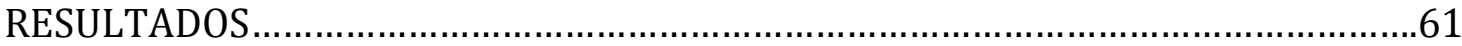

- NÚMERO DE PACIENTES............................................................................62

- EDAD

- TIEMPO QUIRÚRGICO..............................................................................63

- TIEMPO TOTAL DE QUIRÓFANO ...................................................................63

- TIEMPO DE INGRESO ..............................................................................63

- TIEMPO DE INGRESO EN UCIN ....................................................................64

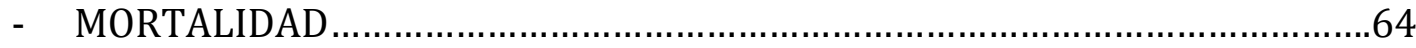

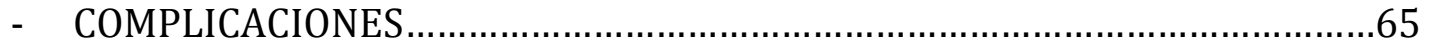

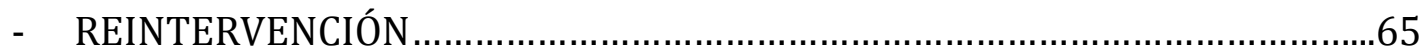

- LUGAR DE LA CIRUGÍA................................................................................66 


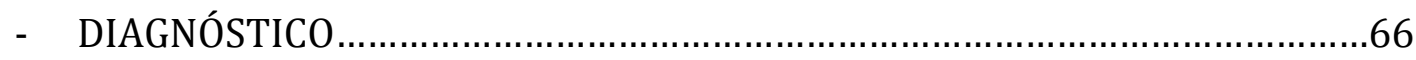

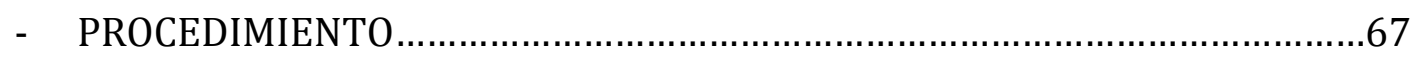

- RELACIÓN ENTRE TIEMPO QUIRÚRGICO Y MORTALIDAD ............................67

- RELACIÓN ENTRE TIEMPO TOTAL DE QUIRÓFANO Y MORTALIDAD...........68

- RELACIÓN ENTRE TIEMPO QUIRÚRGICO Y TIEMPO DE INGRESO ................69

- RELACIÓN ENTRE TIEMPO TOTAL DE QUIRÓFANO Y TIEMPO DE

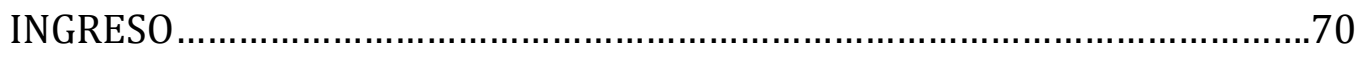

- RELACIÓN ENTRE TIEMPO QUIRÚRGICO Y TIEMPO DE INGRESO EN UCIN

- RELACIÓN ENTRE TIEMPO TOTAL DE QUIRÓFANO Y TIEMPO DE INGRESO EN UCIN

- RELACIÓN ENTRE TIEMPO QUIRÚRGICO Y COMPLICACIONES.....................73

- RELACIÓN ENTRE TIEMPO TOTAL DE QUIRÓFANO Y

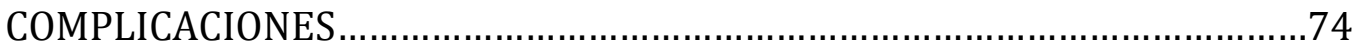

- RELACIÓN ENTRE TIEMPO QUIRÚRGICO Y REINTERVENCIÓN.....................75

- RELACIÓN ENTRE TIEMPO TOTAL DE QUIRÓFANO Y

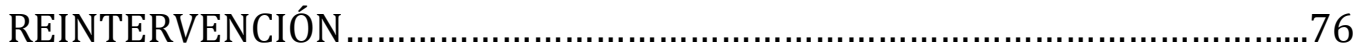

- RELACIONES ANTERIORMENTE ESTABLECIDAS EN LOS PACIENTES QUE RECIBIERON UNA LAPAROTOMÍA .77

- RELACIONES ANTERIORMENTE ESTABLECIDAS EN LOS PACIENTES QUE RECIBIERON UNA TORACOTOMÍA............................................................82

- RELACIONES ANTERIORMENTE ESTABLECIDAS EN LOS PACIENTES

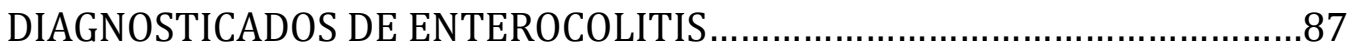

- RELACIONES ANTERIORMENTE ESTABLECIDAS EN LOS PACIENTES DIAGNOSTICADOS DE DUCTUS ARTERIOSO PERSISTENTE .92 
- RELACIONES ANTERIORMENTE ESTABLECIDAS EN LOS PACIENTES

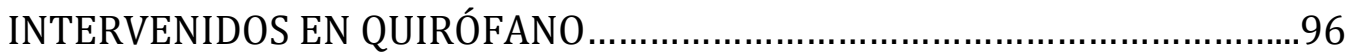

- RELACIONES ANTERIORMENTE ESTABLECIDAS EN LOS PACIENTES

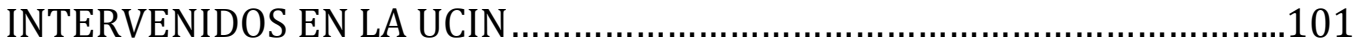

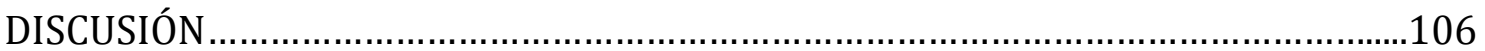

- $\quad$ RELACIÓN ENTRE TIEMPO QUIRÚRGICO Y MORTALIDAD .........................107

- $\quad$ RELACIÓN ENTRE TIEMPO TOTAL DE QUIRÓFANO Y MORTALIDAD .......108

- $\quad$ RELACIÓN ENTRE TIEMPO QUIRÚRGICO Y TIEMPO DE INGRESO.............109

- $\quad$ RELACIÓN ENTRE TIEMPO TOTAL DE QUIRÓFANO Y TIEMPO DE INGRESO

- $\quad$ RELACIÓN ENTRE TIEMPO QUIRÚRGICO Y TIEMPO DE INGRESO EN UCIN

- RELACIÓN ENTRE TIEMPO TOTAL DE QUIRÓFANO Y TIEMPO DE INGRESO EN UCIN

- RELACIÓN ENTRE TIEMPO QUIRÚRGICO Y COMPLICACIONES. 112

- RELACIÓN ENTRE TIEMPO TOTAL DE QUIRÓFANO Y COMPLICACIONES

- RELACIÓN ENTRE TIEMPO QUIRÚRGICO Y REINTERVENCIÓN. 113

- $\quad$ RELACIÓN ENTRE TIEMPO TOTAL DE QUIRÓFANO Y REINTERVENCIÓN 114

- $\quad$ ANÁLISIS DE LOS PACIENTES QUE RECIBIERON UNA LAPAROTOMÍA.....114

- $\quad$ ANÁLISIS DE LOS PACIENTES QUE RECIBIERON UNA TORACOTOMÍA....115

- $\quad$ ANÁLISIS DE LOS PACIENTES DIAGNOSTICADOS DE ENTEROCOLITIS....116

- $\quad$ ANÁLISIS DE LOS PACIENTES DIAGNOSTICADOS DE DUCTUS ARTERIOSO PERSISTENTE 
- $\quad$ ANÁLISIS DE LOS PACIENTES INTERVENIDOS EN QUIRÓFANO................118

- ANÁLISIS DE LOS PACIENTES INTERVENIDOS EN LA UCIN ........................119

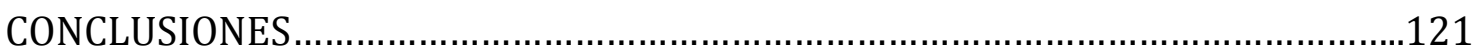

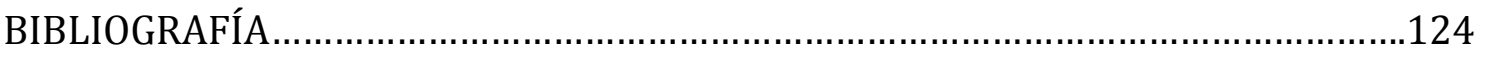

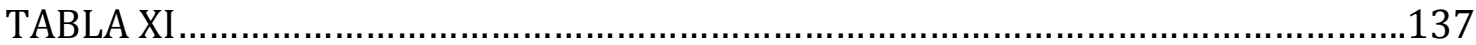




\section{INTRODUCCIÓN:}




\section{CONCEPTO Y JUSTIFICACIÓN DE LA CIRUGÍA PEDIÁTRICA COMO \\ ESPECIALIDAD}

\subsection{DEFINICIÓN:}

Es la especialidad que se ocupa de los procedimientos quirúrgicos en el niño (1), considerando a este desde su nacimiento hasta el fin de la adolescencia, cuando alcanza la completa madurez física y psíquica.

\subsection{JUSTIFICACIÓN DE LA CIRUGÍA PEDIÁTRICA COMO ESPECIALIDAD:}

En una medicina especializada, en la que las subdivisiones se han realizado por aparatos o sistemas es difícil explicar la necesidad de una especialidad como la Cirugía Pediátrica. La clave está en que el niño, como dijo Dickens, no puede considerarse un adulto en miniatura (1). Esto se debe a tres razones fundamentales:

\section{Por las enfermedades tratadas:}

\subsubsection{ESPECIFICIDAD DE LAS ENFERMEDADES QUIRÚRGICAS EN PEDIATRÍA}

Por un lado, existen enfermedades que son exclusivas de la infancia o incluso de periodos muy concretos dentro de la misma. Un ejemplo es la enterocolitis necrotizante del recién nacido pretérmino, otro la estenosis hipertrófica de píloro que afecta a recién nacidos o lactantes o la invaginación intestinal, propia de los niños entre los seis meses y los 2 años $(1,2,3,4)$.

\subsubsection{DIFERENCIAS EN EL MECANISMO PATOGÉNICO}

Por otro lado existen enfermedades que provocan las mismas manifestaciones en niños y en adultos pero cuyo mecanismo es diferente, el paradigma de estas enfermedades es la hernia inguinal, que en el adulto se produce por una debilidad de la pared abdominal y en niños por una persistencia del conducto peritoneovaginal permeable $(1,2,3,4)$. 


\subsubsection{DIFERENCIAS EN LAS MANIFESTACIONES CLÍNICAS Y EVOLUCIÓN}

Finalmente, una misma enfermedad no afecta de la misma forma a adultos o a niños, por ejemplo una apendicitis en un paciente menor de cinco años suele mostrarse de forma diferente al adulto, evolucionando de forma más rápida y mostrándose prácticamente siempre como una peritonitis.

\section{Por las diferencias anatómicas y clínicas entre niño y adulto:}

Existen más razones que justifican la existencia de la cirugía pediátrica como especialidad y todas ellas se basan en la especificidad del niño como paciente. Los niños presentan una anatomía diferente a la del adulto, y no solo por su tamaño (un ejemplo clave podría ser su vía aérea, por su importancia a la hora de intubar), pero además manifiestan de forma diferente sus síntomas y , a determinadas edades, pueden no expresarlos claramente (un dolor abdominal en el lactante se puede mostrar como irritabilidad o encogimiento de piernas $)(5,6)$.

\section{Por la influencia del factor crecimiento:}

Finalmente hay que tener en cuenta que el niño es un paciente en crecimiento y que los tratamientos aplicados deben tener en cuenta el mismo, esto puede ser clave a la hora de tratar por ejemplo una fractura.

\subsection{HISTORIA DE LA CIRUGÍA PEDIÁTRICA COMO ESPECIALIDAD:}

La cirugía pediátrica como especialidad independiente comenzó a desarrollarse durante el siglo XIX y principios del siglo XX. En Europa, al igual que la pediatría, le debe mucho a la fundación de dos grandes hospitales: El Great Ormond Street de Londres y El Hospital de Niños Enfermos de París. En un principio, en Inglaterra, prevalecía la teoría del Dr. West que sostenía que, mientras las enfermedades médicas del niño requerían un tratamiento específico, las quirúrgicas podían tratarse por cirujanos generales. Posteriormente se fue incluyendo dentro de la 
plantilla de los hospitales infantiles personal exclusivamente dedicado al tratamiento quirúrgico de la patología pediátrica.

En Estados Unidos fue el Hospital de niños de Boston el primero en especializarse y de allí son sus primeras dos grandes figuras: el Dr. Ladd y el Dr. Gross (2). En España fue el Hospital Niño Jesús el paradigma de los centros exclusivamente dedicados a la patología del niño y el Hospital La Paz en el que la Cirugía Pediátrica a alcanzado un mayor desarrollo.

\subsection{ESTADO ACTUAL DE LA ESPECIALIDAD}

En la actualidad la Cirugía Pediátrica tiene un desarrollo diferente en las distintas partes del mundo con variaciones incluso dentro de un mismo país.

Por lo general abarca las subespecialidades de Cirugía General Pediátrica, Cirugía Torácica, Urología Pediátrica y Cirugía Plástica Pediátrica.

Sin embargo se recomienda que la Cirugía Cardiaca, la Traumatología y Ortopedia, la Neurocirugía y la Otorrinolaringología sean también llevadas a cabo por especialistas dedicados específicamente a niños.

Sin embargo su campo de actuación no está claramente definido e incluso dentro de un mismo país como es España el rango de edades de los pacientes tratados por un cirujano pediátrico varía de unos hospitales a otros.

Lo mismo ocurre con las patología tratadas, que en muchos casos se superponen entre las distintas especialidades. Por ejemplo un quiste tirogloso puede ser tratado en unos centros por el cirujano pediátrico y en otros por el otorrinolaringólogo.

El campo de la Cirugía Pediátrica ha ido aumentando progresivamente. Esto es debido, en gran parte, al desarrollo de la Anestesia Pediátrica, a la aparición de las 
Unidades de Cuidados Intensivos y de Reanimación exclusivas para niños así como al desarrollo de las especialidades dentro de la pediatría.

A pesar de la subespecialización que caracteriza a los grandes hospitales, quizás el rasgo fundamental de los cirujanos pediátricos es su polivalencia. No en vano es el único cirujano cuyos pacientes pueden variar entre los 500-600g de los prematuros con enterocolitis necrotizante y los $150 \mathrm{~kg}$ de algunos adolescentes con obesidad mórbida. Además, en un mismo parte quirúrgico de un cirujano pediátrico pueden aparecer patologías tan variadas como una criptorquidia, un quiste tirogloso, un labio leporino o un pectus excavatum.

Por otra parte muchas patologías tratadas por esta especialidad requieren tratamientos diferentes a la cirugía ya sean farmacológicos , ortopédicos o incluso relacionados con la rehabilitación. Así, los hemangiomas infantiles que previamente se trataban mediante extirpación ahora se tratan con beta bloqueantes o la enuresis nocturna de los niños se trata con desmopresina. El desarrollo de la urodinámica y el biofeedback para el tratamiento de los trastornos urinarios es un ejemplo de medidas de rehabilitación y las ortesis craneales de las plagiocefalias y los corsés del pectus carinatum son ejemplos de tratamientos ortopédicos.

Pese a la variedad de la patología tratada por los cirujanos pediátricos y a la variabilidad en las edades de los pacientes quizás sean las malformaciones congénitas las enfermedades más específicas de la especialidad, por ello se dice que la Cirugía Pediátrica es una cirugía esencialmente reconstructiva, frente a otras especialidades en las que prima la cirugía oncológica que podría considerarse como una cirugía mutilante. 
Finalmente, si hablamos de un paciente que represente la esencia de la Cirugía Pediátrica ese es el neonato, cuyo manejo difiere enormemente no solo del adulto , si no también del resto de pacientes tratados por nuestra especialidad. Es por ello que vamos a dedicar un apartado en esta introducción exclusivamente al recién nacido y a la cirugía neonatal, pues son los pacientes en los que se centra nuestro estudio . 


\section{EL NEONATO COMO PACIENTE}

Se considera recién nacido al paciente con una edad comprendida entre el nacimiento y los 28 días de vida (5). Presenta unas características específicas y rápidamente cambiantes. Esto se debe a que tiene que adaptarse al cambio entre medio intra y extrauterino.

\subsection{CLASIFICACIÓN DEL RECIÉN NACIDO}

Suelen clasificarse por su edad gestacional y peso.

\section{Según la edad gestacional se dividen en: (5)}

- Pretérmino: (o prematuro)Si nace antes de las 37 semanas de gestación

- A término: Si nace entre las 37 y 42 semanas de gestación

- Postérmino: Si nace a partir de las 42 semanas

\section{Según su peso los recién nacidos se clasifican en: (5)}

- Bajo peso para su edad gestacional: Si están por debajo del percentil 10

- Adecuado peso para su edad gestacional: Si se encuentran entre el percentil 10 y el 90

- Elevado peso para su edad gestacional: Si superan el percentil 90

\subsection{GRUPOS DE ALTO RIESGO:}

De todos estos pacientes los recién nacidos prematuros y los de bajo peso para su edad gestacional son los que presentan un manejo más difícil. Algunos problemas fundamentales de un neonato prematuro son $(5,7,8)$ :

- Reflejo de succión débil

- Absorción intestinal inadecuada

- $\quad$ Riesgo de hipotermia

- Riesgo de hemorragia intraventricular

- Ductus Arterioso Permeable 
- Enfermedad de Membrana Hialina

- Apnea

- Hiperbilirrubinemia

En la siguiente tabla se muestran los riesgos anestésicos más importantes debidos a la prematuridad (7):

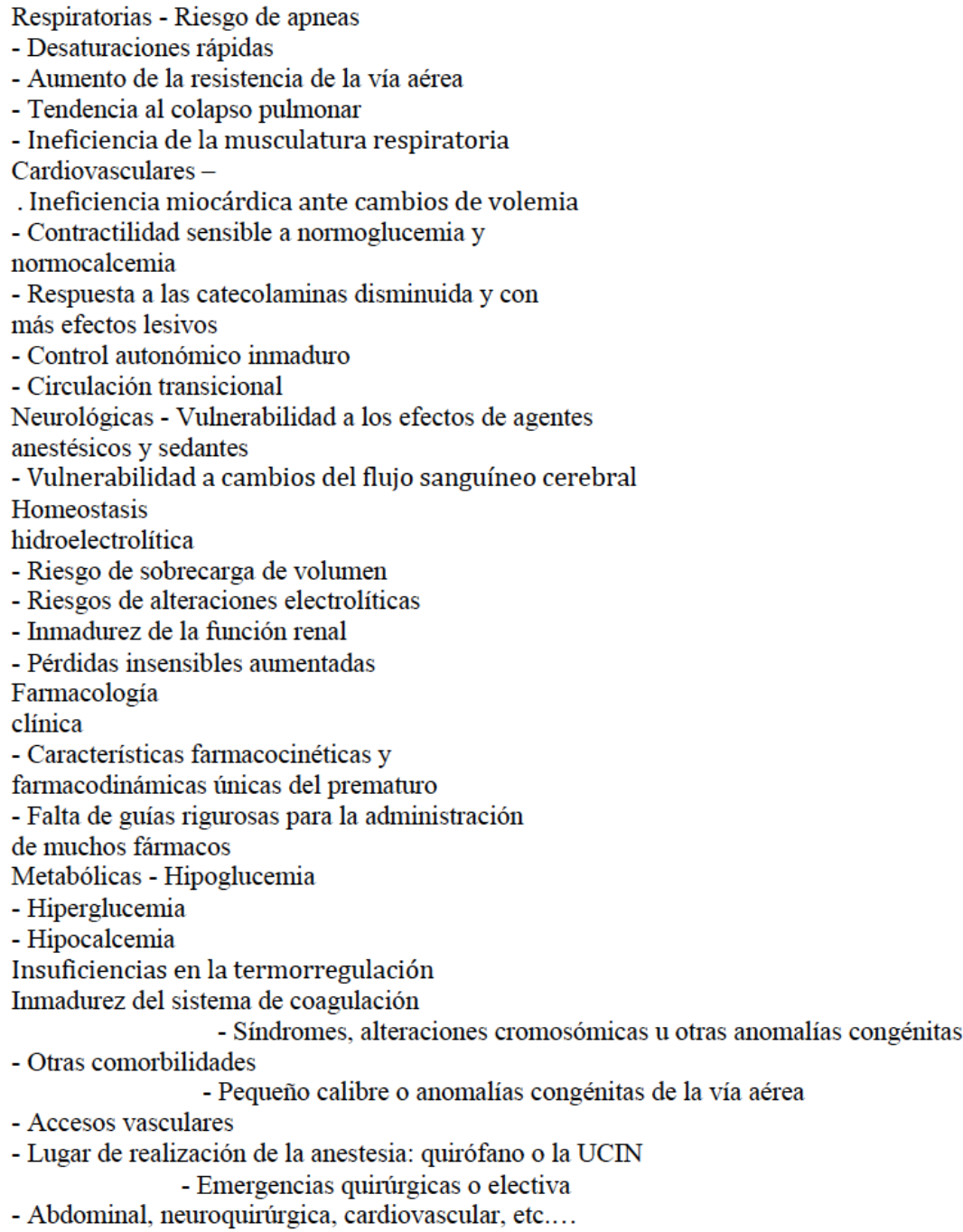

TABLA I: RIESGOS RELACIONADOS CON LA PREMATURIDAD

Los recién nacidos de bajo peso para su edad gestacional por otra parte

presentan mayor riesgo de hipotermia, hipoglucemia y policitemia $(5,8)$. 


\subsection{FACTORES DE VULNERABILIDAD DEL RECIEN NACIDO}

\subsubsection{ALTERACIONES METABÓLICAS:}

El neonato, en su adaptación al medio extrauterino, requiere cambios metabólicos que pueden afectarle sobre todo si va a ser sometido a una intervención quirúrgica $(2,4,8)$ y al periodo de ayuno prolongado que la precede y la sigue.

De hecho son frecuentes tanto los problemas de hipoglucemia como las hiperglucemias $(2,4,5,8)$ y por ello los niveles de glucosa deben monitorizarse con frecuencia y corregirse cuando se precisa.

\subsubsection{RIESGO DE HEMORRAGIA:}

Otro punto fundamental en el neonato que se somete a cirugía es el del sangrado.

El volumen sanguíneo estimado en un recién nacido pretérmino es de 85$100 \mathrm{ml} / \mathrm{kg}$, en un neonato a término es de $85 \mathrm{ml} / \mathrm{kg}$, en el lactante de un mes de $75 \mathrm{ml} / \mathrm{kg}$ y en el de 3 meses de $70 \mathrm{ml} / \mathrm{kg}$, igual que en el adulto (8).

Un recién nacido puede presentar policitemia por trasfusión placentaria, toxemia durante el embarazo, diabetes gestacional o por tener bajo peso para su edad gestacional. Por otra parte también es frecuente la anemia cuyas causas pueden ser hemolítica, por sangrado o por prematuridad entre otras $(5,8)$.

El problema fundamental de un sangrado quirúrgico en un recién nacido es el escaso margen de maniobra ya que pese a tener un volumen sanguíneo relativamente mayor al del adulto su cantidad de sangre total es escasa y a menudo hemorragias que pueden parecer "discretas" producen un significativo 
descenso de la volemia por lo que es fundamental saber valorar cuando un sangrado es importante y saber identificar los signos de shock hipovolémico en estos pacientes.

\subsubsection{RIESGO DE HIPOTERMIA:}

Otro punto importante es el de la termorregulación. El recién nacido presenta una mayor dificultad para conservar su temperatura corporal debido a tres razones fundamentales:

- Área superficial relativamente grande

- Pobre aislamiento

- $\quad$ Bajo peso (8)

La pérdida de calor puede producirse por:

- Evaporación: Si el paciente está húmedo o en contacto con una superficie húmeda.

- Conducción: Por el contacto de la piel con una superficie fría

- Convección: Corrientes de aire que fluyen hacia el sujeto

- Radiación: Pérdida de calor desde el paciente hacia una superficie fría que no está en contacto directo con él $(4,8)$.

El recién nacido compensa esta pérdida de calor mediante un aumento del metabolismo (como es el caso del temblor) o a través del uso de la grasa parda. Este último sistema puede inactivarse con ciertos fármacos como los vasopresores o algunos anestésicos o por la desnutrición.

La termoneutralidad (ambiente térmico óptimo para el recién nacido) varía según la edad gestacional, peso y edad del paciente y por ello es necesario regular la temperatura de las incubadoras según los mismos (8). 
La incapacidad de mantener la neutralidad térmica tiene consecuencias metabólicas y fisiológicas importantes y por ello es necesario mantener la temperatura adecuada del paciente en quirófano.

\subsubsection{DESHIDRATACIÓN:}

El manejo de los líquidos y electrolitos también es diferente en el recién nacido debido a los estrechos márgenes de maniobra. Para determinar los requerimientos de líquidos es necesario conocer:

- El déficit o exceso de líquidos existente

- La demanda metabólica

- Las pérdidas

Debido a que estos factores cambian constantemente en el neonato enfermo es necesario un ajuste constante de los aportes (8).

\subsubsection{RIESGO DE INFECCIÓN:}

El neonato presenta una mayor vulnerabilidad a las infecciones debido a la inmadurez de su sistema inmune que compensa en parte adquiriendo anticuerpos a través de la lactancia materna. Si estos aportes se ven interrumpidos el riesgo de infección se multiplica (5). 


\section{FACTORES PRONÓSTICO EN CIRUGIA NEONATAL}

\subsection{FACTORES DE RIESGO EN EL RECIEN NACIDO EN GENERAL}

Desde hace tiempo se están analizando los factores de riesgo que repercuten en la morbilidad y mortalidad de los recién nacidos, especialmente los pacientes prematuros(9,10,11,12,13,14). Así, se han desarrollado distintas tablas que ayudan a valorar el riesgo de mortalidad en estos pacientes $(14,15,16,17,18)$. Las más importantes son las siguientes:

- Clínical Risk Index for Babies (CRIB) : que valora el peso, la edad gestacional, la presencia de malformaciones congénitas, exceso de bases máximo en las primeras 12 horas y FiO2 máxima y mínima apropiadas en las primeras 12 horas (14). En la siguiente tabla se muestra este índice de riesgo:

\begin{tabular}{|c|c|}
\hline Variable & Score \\
\hline \multicolumn{2}{|l|}{ Birthweight (g) } \\
\hline$>1,350$ & 0 \\
\hline $851-1,350$ & 1 \\
\hline $701-850$ & 4 \\
\hline$<700$ & 7 \\
\hline \multicolumn{2}{|l|}{ Gestational age (weeks) } \\
\hline$>24$ & 0 \\
\hline$<24$ & 1 \\
\hline \multicolumn{2}{|l|}{ Congenital malformation } \\
\hline None & 0 \\
\hline No imminent life threatening & 1 \\
\hline With imminent life threatening & 3 \\
\hline \multicolumn{2}{|c|}{ Maximum BE during first $12 \mathrm{~h}$ of life $(\mathrm{mmol} / \mathrm{l})$} \\
\hline$>-7.0$ & 0 \\
\hline-7.0 to -9.9 & 1 \\
\hline-10.0 to -14.9 & 2 \\
\hline$<-15.0$ & 3 \\
\hline \multicolumn{2}{|c|}{ Minimal appropriate $\mathrm{FiO} 2$ during first $12 \mathrm{~h}$ of life } \\
\hline$<0.40$ & 0 \\
\hline $0.41-0.60$ & 2 \\
\hline $0.61-0.90$ & 3 \\
\hline $0.91-1.00$ & 4 \\
\hline \multicolumn{2}{|c|}{ Maximum appropriate $\mathrm{FiO} 2$ during first $12 \mathrm{~h}$ of life } \\
\hline$<0.40$ & $\mathbf{0}$ \\
\hline $0.41-0.80$ & 1 \\
\hline $0.81-0.90$ & 3 \\
\hline $0.91-1.00$ & 5 \\
\hline
\end{tabular}

TABLA II: ÍNDICE DE RIESGO CRIB 
- Clínical Risk Index for Babies II (CRIB II): Variante del anterior que incluye una diferenciación por sexos e introduce la temperatura como factor pronóstico (15).

- $\quad$ Score of Neonatal Acute Pathology (SNAP): que analiza parámetros cardiacos, respiratorios así como múltiples valores de laboratorio (16).

- Score of Neonatal Acute Pathology- Perinatal Extension (SNAPPE)

- SNAP II . Actualización del SNAP (18) cuyos valores aparecen en la siguiente tabla:

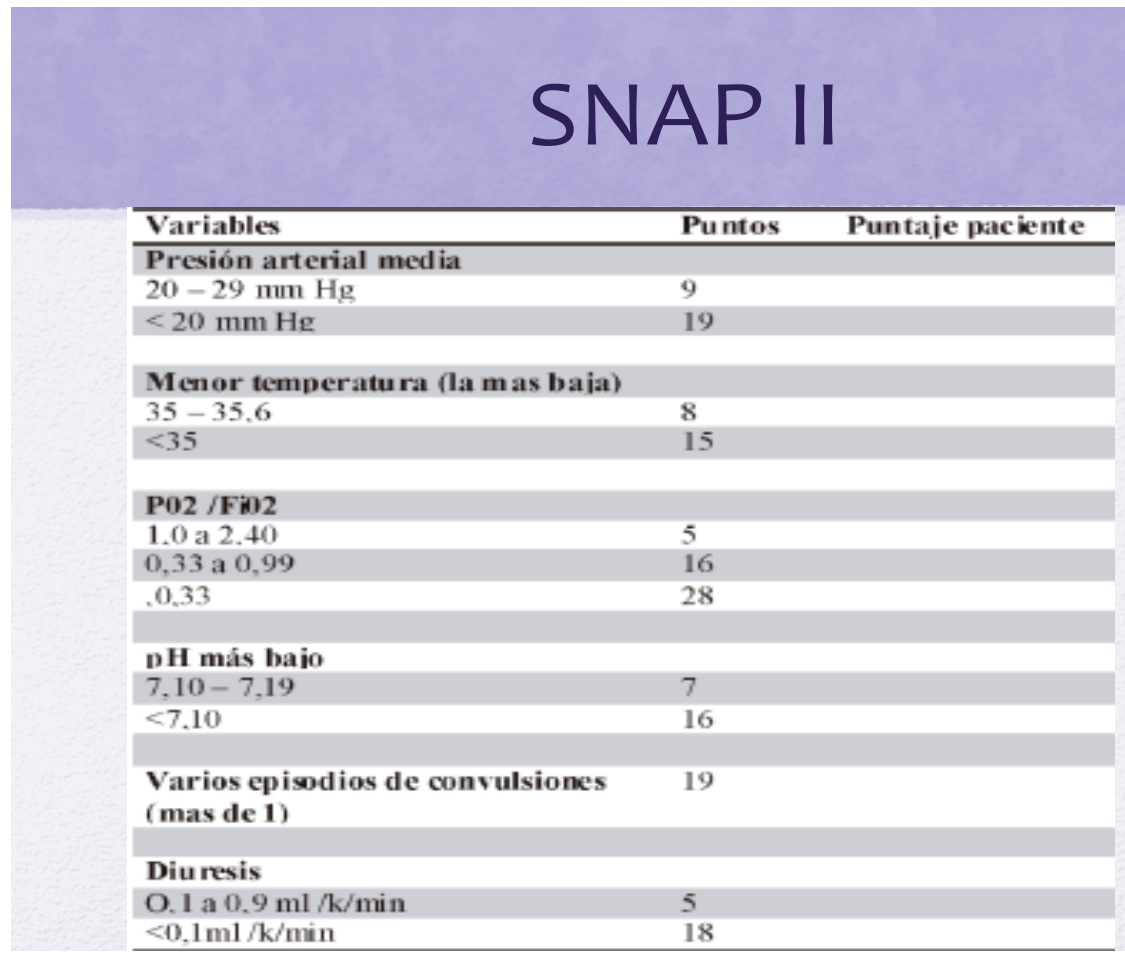

TABLAIII: ÍNDICE DE RIESGO SNAP II 
- $\quad$ SNAPPE II . Al igual que el SNAPPE añade al SNAP el SNAPPE II añade al SNAP II las siguientes variables de estudio que se muestran en la tabla (18):

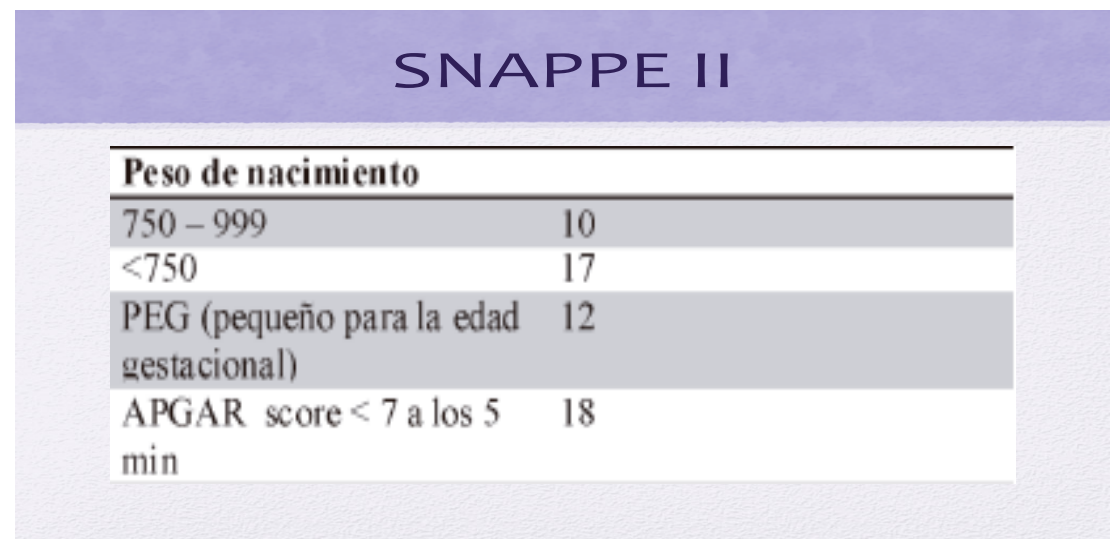

TABLA IV: VARIABLES AÑADIDAS AL SNAP II PARA DETERMINAR EL SNAPPE II

- National Index of Child Health and Human Development ( NICHHD)

- Berlin Score

- Neonatal Mortality Prognosis Index (NMPI)

Los factores analizados en los anteriores índices de riesgo pueden verse resumidos en la siguiente tabla $(19,20,21,22,23,24,25)$ : 


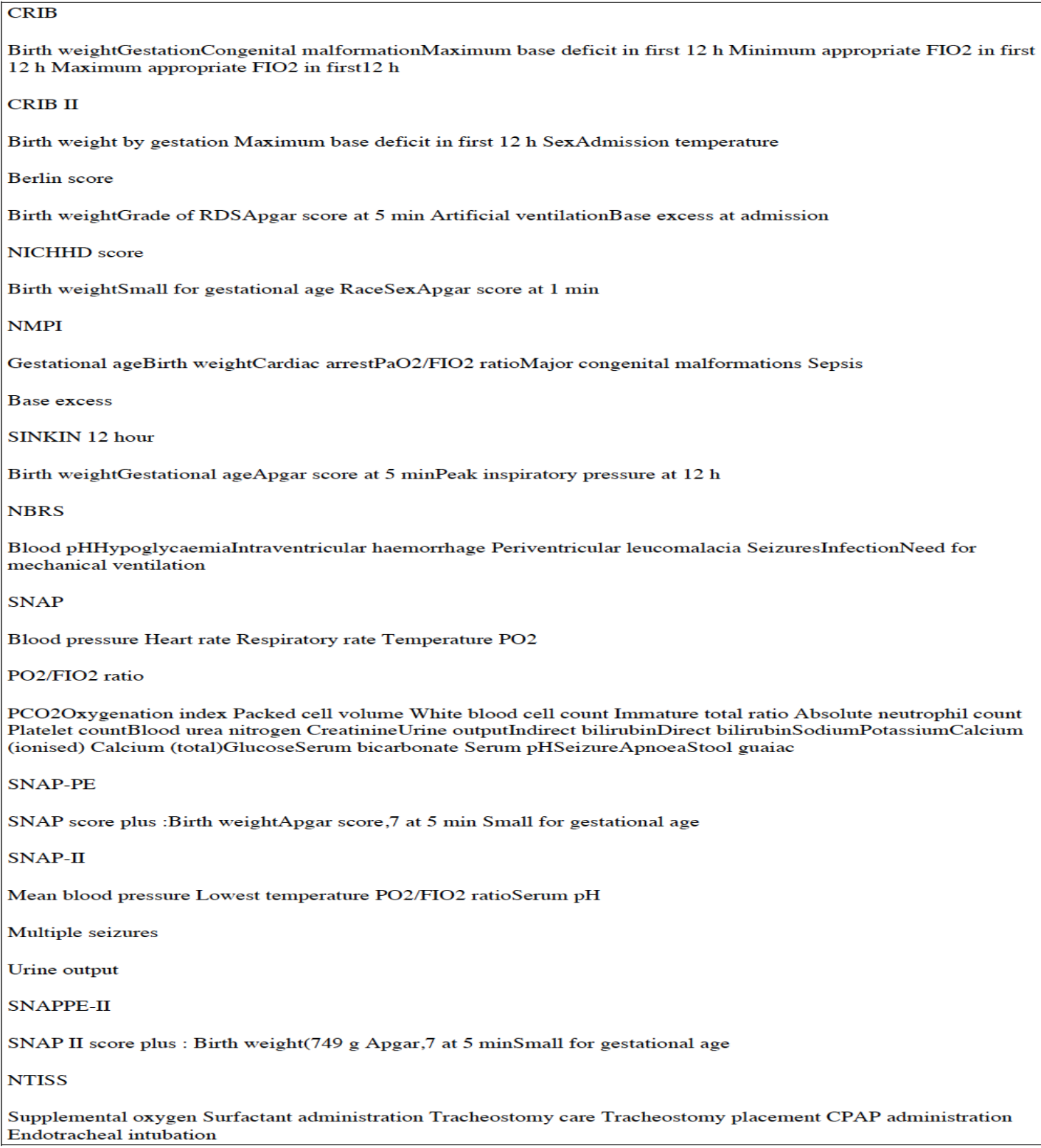

Mechanical ventilationMechanical ventilation with paralysis High frequency ventilation Extracorporeal membrane oxygenation Indomethacin administrationVolume expansionVasopressor administrationPacemaker on standbyPacemaker usedCardiopulmonary resuscitation AntibioticsDiuretics (enteral)Steroids (postnatal)AnticonvulsantAminophyllineOther unscheduled medication Diuretics (parenteral)Treatment of metabolic acidosis Potassium binding resinFrequent vital signsCardiorespiratory monitoring PhlebotomyThermoregulated environment Non-invasive oxygen monitoring Arterial pressure monitoringCVP monitoringUrinary catheterQuantitative intake and outputGavage feedingIntravenous fat emulsionIntravenous amino acid solution PhototherapyInsulin administrationPotassium infusionTransfusionIntravenous c globulinRed blood cell transfusionPartial volume exchange transfusion Platelet transfusionWhite blood cell transfusionDouble blood cell transfusionTransport of patientChest tubeMinor operationThoracentesisMajor operationPericardiocentesisPericardial tubeDialysisVascular accessPeripheral intravenous lineArterial lineCentral venous line

CRIB, clinical risk index for babies; FIO2, fractional inspired concentration of oxygen; NBRS, neurobiological risk score; NMPI, neonatal mortality prognosis index; NTISS, neonatal therapeutic intervention scoring system; $\mathrm{PO}$, partial pressure of oxygen; RDS, respiratory distress syndrome; SNAP, score for neonatal acute physiology; SNAP$\mathrm{PE}$, score for neonatal acute physiology-perinatal extension.

TABLA V: FACTORES ANALIZADOS EN LOS ÍNDICES DE RIESGO NEONATAL 


\subsection{FACTORES DE RIESGO DEL RECIÉN NACIDO QUIRÚRGICO:}

Los factores pronóstico que afectan a los pacientes recién nacidos que se someten a una intervención quirúrgica no están tan bien estudiados.

El primer estudio que valoró factores de riesgo que afectaban a la mortalidad en neonatos que iban a someterse a cirugía lo realizó Waterson sobre la atresia de esófago. Los factores que analizó fueron el peso del paciente y la presencia de malformaciones congénitas asociadas o neumonía $(2,3,4,26)$. Dichos criterios aparecen en la siguiente tabla:

\section{CRITERIOS DE} WATERSON

\begin{tabular}{lll} 
& Waterston Supervivencia (\%) \\
\hline PRN $>2.500$ g, sanos & A & 100 \\
\hline $\begin{array}{l}\text { PRN 2.000-2.500 g, neumonía } \\
\text { leve-moderada 0 anomalías } \\
\text { cardiacas moderadas }\end{array}$ & B & 85 \\
\hline $\begin{array}{l}\text { PRN }<2.000 \text { g, neumonía } \\
\text { severa } 0 \text { malformación } \\
\text { cardiaca grave }\end{array}$ & $\mathrm{C}$ & 65 \\
\hline
\end{tabular}

TABLA VI: CRITERIOS DE WATERSON

Posteriormente estos criterios han sido modificados por la clasificación de Spitz $(2,3,4,27)$ y Montreal $(2,3,4,28,29,30)$ que aparecen en las tablas que se muestran a continuación: 


\section{CRITERIOS DE SPITZ}

\begin{tabular}{lcc} 
& Spitz & Supervivencia (\%) \\
\hline PRN $>1.500 \mathrm{~g}$, sanos & $\mathrm{I}$ & 97 \\
\hline $\begin{array}{l}\text { PRN }<1.500 \mathrm{~g}, 0 \text { malformación } \\
\text { cardiaca mayor }\end{array}$ & $\mathrm{II}$ & 59 \\
\hline $\begin{array}{l}\text { PRN }<1.500 \text { g y malformación } \\
\text { cardiaca mayor asociada }\end{array}$ & III & 22 \\
\hline $\begin{array}{l}\text { PRN: peso del recién nacido. } \\
\end{array}$ & & \\
\hline
\end{tabular}

TABLA VII: CITERIOS DE SPITZ

\section{CLASIFICACIÓN DE MONTREAL}

\author{
BUEN PRONÓSTICO \\ - No dependencia del \\ ventilador \\ No malformaciones \\ asociadas asociadas \\ SUPERVIVENCIA: \\ $90-95 \%$
}

\author{
MAL PRONÓSTICO \\ sí dependencia del \\ respirador \\ - Sí malformaciones \\ asociadas \\ SUPERVIVENCIA: \\ $20-37 \%$
}

TABLA VIII: CRITERIOS DE MONTREAL

También se han estudiado factores pronóstico para la hernia diafragmática o para la atresia intestinal.

Finalmente se han estudiado de forma aislada distintas características pre y postnatales que pueden influir en el pronóstico de cada patología por separado , sobre todo en enterocolitis necrotizante $(31,32,33,34,35)$, hernia diafragmática $(36,37)$ atresia intestinal $(38,39)$ y gastrosquisis $(40)$. Muy poco 
hay publicado, sin embargo sobre factores pronóstico en cirugía neonatal en general $(41,42)$ y prácticamente nada que analice de forma específica el tiempo quirúrgico.

Dentro de estos artículos debemos destacar cuatro:

\section{- MANCHADA Y COLABORADORES (43):}

Analizan durante un período de 15 meses parámetros clínicos y bioquímicos y su influencia en el pronóstico de 165 neonatos intervenidos en su unidad. Con ello pretenden elaborar un índice de riesgo para el paciente recién nacido que precise cirugía. Manchada concluye que una edad gestacional corta, y la presencia de signos de distress respiratorio y shock en el momento del ingreso empeoran el pronóstico de los pacientes:

- La edad gestacional :es un factor pronóstico importante en neonatología en general. Aparece de forma repetida en casi todos los índices de riesgo neonatal y como es obvio también debe influir en la evolución de los pacientes quirúrgicos.

- Las alteraciones del ritmo cardiaco, propias de la sepsis y el shock también mostraron significación en el análisis multivariante

- El distress respiratorio fue la tercera variable clínica que afectó de forma importante al pronóstico y que también es una constante en los índices de riesgo neonatales.

- El test de APGAR no pudo analizarse pues gran parte de los partos fueron domiciliarios. 
- Tampoco pudo analizarse la influencia de las malformaciones asociadas, sobre todo cardiacas que podrían afectar al pronóstico como ocurre en los criterios de Waterson.

- En cuanto a los parámetros bioquímicos o hematológicos, aquellos relacionados con la sepsis, el distress respiratorio y el shock fueron los que mostraron asociación con el pronóstico. No así los parámetros de función hepática o renal, esta última seguramente porque en las primeras $48 \mathrm{~h}$ correspondería más bien a la función renal materna que a la del neonato.

Quizás una de las mayores limitaciones de este estudio es que gran parte de los partos fueron domiciliarios y que no existe un seguimiento prenatal de los pacientes , lo que ayudaría a una atención precoz en caso de malformación congénita severa o parto prematuro. Esto hace que la población objeto de estudio pueda no ser comparable a la existente en nuestro país. Por otra parte no alcanza su objetivo de desarrollar un índice pronóstico para los recién nacidos que precisen cirugía.

\section{- UGWU Y COLABORADORES (44):}

Concluyen en su artículo que la mortalidad es mayor en pacientes pretérmino y en aquellos que nacieron fuera del hospital. Estas conclusiones coinciden en gran parte con el estudio de Manchada y al igual que el anterior, el estudio se lleva a cabo sobre una población con escaso seguimiento prenatal y muchos partos domiciliarios.

\section{- D. CATRE Y COLABORADORES(45):}

En su artículo sobre factores predictivos de morbilidad severa en neonatos intervenidos concluyen que las reintervenciones, la cirugía por hernia 
diafragmática congénita, la cirugía abdominal y la edad gestacional inferior a 32 semanas están relacionados con una mayor morbilidad. En este primer artículo que comentamos de dicho autor habla sobre complicaciones graves a corto plazo y no sobre mortalidad.

- Edad gestacional : parece claro que es un factor de riesgo en neonatos que van a someterse a cirugía pues aparece en todos los estudios, al igual que Manchada el bajo peso es factor de riesgo en los test univariante pero no en los multivariante cuando se separa de la edad gestacional.

- La cirugía por hernia diafragmática o la cirugía abdominal en general: también son factores de riesgo y en menor medida los defectos de pared abdominal, la enterocolitis necrotizante o las malformaciones anorrectales.

- El tiempo quirúrgico lo divide en 2 grupos: cirugías de más de 2 horas y cirugías de menos de 2 horas y no encuentra relación estadísticamente significativa con las complicaciones.

Quizá la mayor diferencia entre la población de su estudio y del nuestro es que no incluye pacientes cardiópatas lo que le resta complejidad a su población. Además este tipo de pacientes, podrían ser más sensibles a una prolongación de la intervención.

\section{- D. CATRE Y COLABORADORES (46):}

Finalmente este mismo autor analiza en otro artículo los factores de riesgo perioperatorios que influyen en la mortalidad en los 30 siguientes días a la cirugía en recién nacidos y concluye que una puntuación mayor de 3 en la American Society of Anesthesiologist (ASA) score y la cirugía por 
enterocolitis necrotizante o perforación intestinal están relacionados con una mayor mortalidad. En la siguiente tabla se muestra la clasificación ASA

\section{ASA CRITERIA}

\begin{tabular}{|c|c|c|c|}
\hline Clase & $\begin{array}{c}\text { Descripción de la Clase Funcional } \\
\text { nyha }\end{array}$ & Condición Clínica & $\begin{array}{l}\text { Mortalidad } \\
\text { Operatoria }\end{array}$ \\
\hline (1) & $\begin{array}{l}\text { Pacientes con cardiopatía pero sin } \\
\text { limitaciones resultantes de la actividad } \\
\text { fisica. La actividad fisica ordinaria no } \\
\text { causa sintomas cardiacos. Dolor, } \\
\text { disnea, palpitaciones, sincope }\end{array}$ & Paciente Sano & $4.3 \%$ \\
\hline $\begin{array}{l}\text { ASA } \\
\text { (2) }\end{array}$ & $\begin{array}{l}\text { Pacientes con enfermedades cardiacas } \\
\text { que producen cierta Limitación en la } \\
\text { actividad física. Están cómodos en } \\
\text { reposo pero de ordinario la actividad } \\
\text { produce sintomas cardiacos. } \\
\text { Alteraciones sistémicas leves a } \\
\text { Moderadas. }\end{array}$ & $\begin{array}{l}\text { HTA y Diabetes controlada. Anemia, } \\
\text { Tabaquismo. Asma. Embarazo. Obesidad. } \\
\text { Menores de } 1 \text { año. Mayores de } 70 \text { años. }\end{array}$ & $10.6 \%$ \\
\hline (3) & $\begin{array}{l}\text { Pacientes con cardiopatías que } \\
\text { producen limitación muy manifiesta en } \\
\text { la actividad fisica. Están cómodos en } \\
\text { reposo pero una actividad menor que la } \\
\text { ordinaria produce sintomas. }\end{array}$ & $\begin{array}{c}\text { Angor. HTA y Diabetes no controlada. } \\
\text { Asma. EPOC. Obesidad Mórbida. Historia } \\
\text { de IM. }\end{array}$ & $25.0 \%$ \\
\hline (4) & $\begin{array}{l}\text { Pacientes cardiópatas con incapacidad } \\
\text { para realizar cualquier actividad física } \\
\text { sin incomodidad. Sintomáticos en } \\
\text { reposo }\end{array}$ & $\begin{array}{c}\text { Angor Inestable. Insuficiencia } \\
\text { Respiratoria o Cardiaca. } \\
\text { Hepatopatía.Insuficiencia Renal. }\end{array}$ & $67.0 \%$ \\
\hline
\end{tabular}

TABLA IX: CRITERIOS ASA

Dicha clasificación es difícil de adaptar a las características del recién nacido (41) por lo que los anestesistas de los distintos centros realizan interpretaciones o adaptaciones individualizadas.

Sólo en este ultimo artículo se menciona el tiempo quirúrgico como factor de riesgo de mortalidad y al igual que en el anterior se limita a discernir entre cirugías de más de dos horas y cirugías más cortas.

Al igual que en el caso anterior existen diferencias entre su población a estudio y la nuestra ya que el número de enterocolitis y el número de ductus arteriosos 
intervenidos es significativamente menor lo que sugiere una mayor edad gestacional. Por otra parte su estudio carece de pacientes cardiópatas, muy frecuentes en nuestro centro por ser referencia para ese tipo de patología. 


\section{TIEMPO QUIRÚRGICO COMO FACTOR DE RIESGO}

\subsection{ETAPAS DE LA CIRUGÍA:}

\section{- Etapa inicial:}

Durante los primeros años del desarrollo de la cirugía la duración del procedimiento quirúrgico era fundamental para el pronóstico final. Esto era en gran parte debido al dolor que produce la intervención, al sangrado, a la hipotermia y a la posibilidad de infección postoperatoria. El problema se ponía de manifiesto fundamentalmente en el caso de la cirugía de guerra.

\section{-Segunda etapa:}

Conforme fue desarrollándose la Anestesia, que permitió un buen control del dolor durante la intervención, con los avances de la asepsia y antisepsia así como las medidas para el control de sangrado y las trasfusiones el tiempo quirúrgico ha ido perdiendo importancia como factor de riesgo.

\section{-En la actualidad:}

Pese a estos grandes avances se han descubierto campos dentro de la cirugía donde la prolongación del acto operatorio puede resultar letal para el paciente.

\subsection{CIRUGÍAS ESPECIALMENTE DEPENDIENTES DEL TIEMPO QUIRÚRGICO: HIPOTERMIA Y SANGRADO}

- Cirugía del paciente politraumatizado: El mejor ejemplo para ilustrar la importancia de una cirugía rápida que solucione los problemas más importantes del paciente es la Cirugía de Control de Daños en el paciente politraumatizado. 
Este concepto surgió en los años 70 en los barcos de guerra del ejército americano. Previamente a este momento el cirujano de politrauma era un cirujano especialmente agresivo que intentaba en un único acto quirúrgico solucionar todos los problemas del paciente. El resultado era una cirugía de tiempos muy prolongados en un paciente clínicamente inestable que generalmente sobrevivía a duras penas al acto quirúrgico y que fallecía en las horas siguientes en la Unidad de Cuidados Intesnsivos.

Pero ¿cuál era la causa de la muerte del paciente? Y lo más importante ¿Existía alguna manera de disminuir esta alta tasa de mortalidad?.

Analizando las causas de la elevada mortalidad se descubrió que ésta era en gran parte debida a la triada Hipotermia-Coagulopatía-Acidosis secundaria al propio traumatismo, al sangrado intenso y a la prolongada duración de la intervención quirúrgica .

Surge así un nuevo concepto del manejo del paciente politraumatizado en 3 fases:

- Cirugía inicial

- Fase de estabilización

- Cirugía definitiva

* Cirugía inicial: El primer paso consiste en una cirugía de corta duración cuya misión es controlar la hemorragia y la contaminación, realizar un packing y un cierre temporal de la herida quirúrgica.

La clave de este primer paso es elegir a los pacientes susceptibles de ser sometidos a este tratamiento. Generalmente la decisión se toma ante un sangrado masivo, si el enfermo está hemodinámicamente inestable , si hay coagulopatía, hipotermia o si no se puede cerrar la herida quirúrgica por 
edema. La misión de este paso es controlar la hemorragia y la contaminación mediante medidas temporales y rápidas como pueden ser el packing para el sangrado o el cierre de intestino mediante grapas o sutura.

Finalmente la herida quirúrgica se cierra temporalmente con pinzas de campo o una bolsa de Bogotá.

* Fase de estabilización: El segundo paso es el período de estabilización, cuyos objetivos son alcanzar la homeostasis térmica, corregir la coagulopatía, lograr la estabilidad hemodinámica, realizar un apoyo ventilatorio del paciente e identificar las lesiones para su tratamiento definitivo. Esta segunda fase se lleva a cabo en la Unidad de Cuidados Intensivos y requiere una reevaluación continua del paciente.

* Cirugía definitiva: El tercer paso, una vez estabilizado el paciente e identificadas las lesiones, consiste en el tratamiento quirúrgico definitivo. El paciente debe estar normotérmico, hemodinámicamente estable y en ausencia de coagulopatía. Se lleva a cabo entre las 18 y las 48 h después de la primera cirugía y durante este procedimiento se retira el packing, se identifican y se tratan las lesiones causadas por el traumatismo.

Con este nuevo planteamiento los equipos especializados en atención al paciente politraumatizado han conseguido disminuir la mortalidad en pacientes inestables tras un traumatismo severo. El éxito de estas medidas se basa en gran parte en la rapidez de la primera cirugía que incluso llega a cronometrarse para no superar la hora de duración $(48,49,50,51,52,53,54,55$, $56,57,58,59,60$ ) 
- Cirugía Cardiaca con circulación extracorpórea. En ella la importancia radica fundamentalmente en el tiempo durante el cual el paciente se encuentra en bomba y el tiempo de recuperación de la temperatura normal ya que esto influye en las alteraciones de la coagulación y por tanto en la posibilidad de hemorragia $(61,62,63,64)$.

\subsection{TIEMPO QUIRÚRGICO E INFECCIÓN}

Una vez demostrada la influencia del tiempo quirúrgico en la posibilidad de desarrollar hipotermia en el paciente intervenido (y sus consecuencias negativas sobre el equilibrio metabólico y la coagulación) y aceptado el hecho de que una cirugía más prolongada aumenta el riesgo de sangrado en pacientes especialmente sensibles a ello pasamos a hablar del tercer punto clave relacionado con la prolongación del acto operatorio: la infección. Existen múltiples estudios que abordan los factores de riesgo de desarrollar una infección de herida quirúrgica o de infección en el lecho operatorio. Está demostrado que cuanto más tiempo está expuesto el material quirúrgico en el quirófano más gérmenes colonizan el mismo. Sin embargo esto no es suficiente para concluir que a más duración de la cirugía más infección. Por ello es necesario estudiar si las infecciones propiamente dichas están relacionadas con un tiempo operatorio prolongado. Hay varios artículos que abordan los factores de riesgo de infección postoperatoria en el sitio quirúrgico y la mayor parte de ellos concluyen que hay una relación estadísticamente significativa entre el tiempo quirúrgico prolongado y el riesgo de infección $(65,66,67,68,69,70,71,72)$. 
De hecho el National Nosocomial Infections Surveillance System (NNISS) utiliza un sistema de riesgo ajustado para pronosticar el riesgo de infección quirúgica que incluye tres factores de riesgo:

- $\quad$ ASA score

- Grado de contaminación de la herida quirúrgica (66) , mostrada en la siguiente tabla:

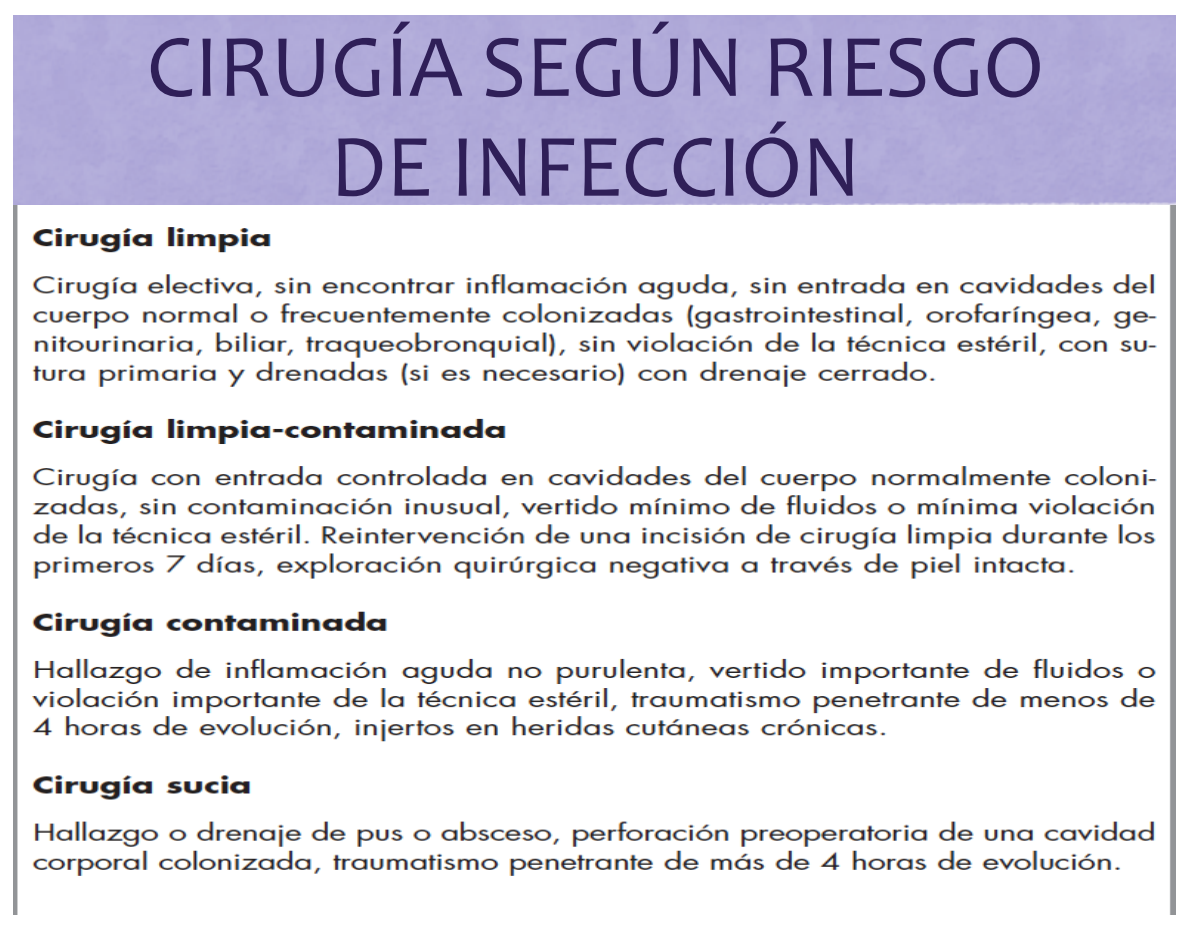

TABLA X: CLASIFICACIÓN DE LA CIRUGÍA POR RIESGO DE INFECCIÓN

- Duración del tiempo quirúrgico de la intervención.

Por tanto, por un lado parece que el tiempo quirúrgico es un factor de riesgo que podría influir en el pronóstico y por tanto en la morbimortalidad de pacientes concretos como son los politraumatizados o pacientes cardiovasculares debido a su mayor sensibilidad al sangrado y a la hipotermia y por otro está demostrado que la duración prolongada de la cirugía está relacionada con un mayor riesgo de infección y por tanto con una mayor 
morbilidad postoperatoria en la cirugía en general. Por todo ello parece necesario analizar si este factor influye en la evolución de un paciente tan complejo como el recién nacido que se ve sometido a una intervención quirúrgica. 


\section{TIEMPO QUIRÚRGICO Y CIRUGÍA NEONATAL}

Por un lado tenemos un paciente con unas características especiales, el neonato.

Los recién nacidos, como ya hemos explicado anteriormente, son especialmente sensibles a la hipotermia y sus consecuencias tanto metabólicas como sobre la coagulación. Esto se debe a su superficie corporal relativamente más grande que en niños mayores o adultos, a su bajo peso y a su déficit de aislamiento (8), características que se ven potenciadas en los niños prematuros o de bajo peso para su edad gestacional.

Además los neonatos son especialmente sensibles a la hemorragia, algunos , como los prematuros tienen una anemia previa al procedimiento (5) y en cualquier caso, pérdidas hemáticas que pueden parecer escasas producen una gran anemización en estos pacientes por su escaso volumen sanguíneo total. Finalmente debido a la inmadurez de sus defensas son especialmente sensibles a las infecciones, este riesgo se ve aumentado si no pueden alimentarse correctamente por lactancia materna (5) como ocurre en gran parte de los pacientes quirúrgicos o si precisan un acceso venoso central prolongado o nutrición parenteral.

Por otro lado sabemos que un tiempo quirúrgico prolongado puede ser causa de mayor sangrado (en pacientes especialmente sensibles a ello) , así como de la triada hipotermia- alteraciones metabólicas- coagulopatía y que existe una relación importante entre la duración del acto quirúrgico y la posibilidad de sufrir una infección en el postoperatorio.

Todo esto justificaría el estudio del tiempo quirúrgico como factor de riesgo en cirugía neonatal. 
Existen muy pocos artículos que mencionen el tiempo quirúrgico como factor pronóstico en Cirugía Neonatal y a parte de la valoración del riesgo de infección en el postoperatorio (72).

Por otra parte, los riesgos antes mencionados de hipotermia, sangrado e infección se han logrado combatir con éxito a través de una serie de medidas que expondremos a continuación por lo que dichos riesgos se han minimizado desde los comienzos de la Cirugía Pediátrica como especialidad hasta la actualidad.

La hipotermia del recién nacido que va a ser intervenido puede prevenirse en el momento actual mediante distintos sistemas:

- La regulación térmica del propio quirófano es mucho más efectiva en la actualidad que hace unos años.

- Existen distintos mecanismos dirigidos a evitar la pérdida de calor a través de la cabeza y extremidades.

- Se ha generalizado el uso de mantas térmicas en cirugía pediátrica

- Los sueros utilizados durante la cirugía se calientan para usarse a la temperatura adecuada.

- Se usan paños impermeabilizados y apósitos plásticos para evitar que el paciente se moje.

- Cada vez son más frecuentes los procedimientos mínimamente invasivos que no exponen la cavidad abdominal evitando pérdidas de temperatura innecesarias.

- Los pacientes clínicamente inestables pueden intervenirse directamente en la Unidad de Cuidados Intensivos Neonatales (UCIN) directamente en la incubadora o en una cuna térmica lo que disminuye el riesgo de hipotermia 
durante la cirugía y también la que pudiera generarse durante el traslado desde dicha Unidad al quirófano $(73,74,75,76,77,78,79)$.

Las alteraciones metabólicas secundarias a la hipotermia se pueden tratar gracias a una detección precoz de las mismas mediante gasometrías que ofrecen un resultado prácticamente instantáneo.

Los problemas de coagulación se han minimizado gracias a las siguientes medidas:

- Medidas para corregir la hipotermia.

- Trasfusión de sangre $(80,81,82,83,84$,$) y plaquetas.$

- Trasfusión de plasma fresco.

- Factores de la coagulación recombinantes $(85,86,87,88,89,90,91,92,93,94)$.

La pérdida de sangre por hemorragia se ha disminuido gracias a los mecanismos que se exponen a continuación:

- Bisturí eléctrico monopolar y bipolar.

- Bisturí armónico.

- Selladores vasculares .

- Clips vasculares de pequeño tamaño.

- Geles hemostáticos.

- Sustratos que favorecen la cascada de la coagulación .

Finalmente los riesgos de infección se han reducido con el desarrollo de los diferentes antibióticos y antifúngicos, medidas de asepsia y antisepsia así como los distintos estudios microbiológicos que tipifican los gérmenes más frecuentes en cada unidad del hospital y establecen las profilaxis antibióticas necesarias. 
Todo lo anteriormente mencionados nos plantea la siguiente pregunta: ¿Sigue siendo el tiempo quirúrgico un factor determinante el la morbilidad y mortalidad postoperatorias en cirugía neonatal?. 


\section{OTROS FACTORES RELACIONADOS CON EL TIEMPO QUIRÚRGICO Y SU INFLUENCIA EN EL PRONÓSTICO:}

Existen también otras variables que sería necesario analizar por su relación con el tiempo operatorio y la morbi-mortalidad postoperatoria en neonatos: - La influencia sobre la evolución del paciente del tiempo total que éste pasa en quirófano: Desde hace años el tiempo que dura la intervención ocupa cada vez una menor proporción del tiempo total del paciente en quirófano ya que los procesos de monitorización , técnicas de anestesia regional y la preparación del neonato para la cirugía ocupan cada vez más tiempo y alargan el período de estancia del mismo en quirófano y, por tanto, el riesgo de hipotermia.

- El tipo de procedimiento: las posibilidades de sangrado o el riego de pérdida de temperatura no es exactamente el mismo en una laparotomía o toracotomía que en una anorrectoplastia por ejemplo.

- El diagnóstico del paciente: Ya que el pronóstico difiere entre una laparotomía por una atresia duodenal que el de una laparotomía por una hernia diafragmática.

- Lugar de intervención: los pacientes intervenidos en la Unidad de Cuidados Intensivos Neonatales sufren menos hipotermia que los operados en quirófano (73) y esto podría suponer un sesgo para el estudio.

Toda esta introducción pone de manifiesto la necesidad de estudiar el tiempo quirúrgico como factor de riesgo en Cirugía Neonatal de forma específica así como de los factores que pueden modificar dicha influencia. 


\section{OBJETIVOS:}


El objetivo principal de este estudio es determinar si el tiempo quirúrgico es un factor de riesgo que influya en la evolución del recién nacido sometido a una cirugía mayor.

Para ello pretendemos:

- Determinar su influencia sobre la mortalidad global de los pacientes así como en grupos concretos:

. Pacientes que recibieron laparotomía.

. Pacientes que recibieron toracotomía.

. Pacientes diagnosticados de Enterocolitis Necrotizante.

. Pacientes diagnosticados de Ductus Arterioso Persistente.

- Averiguar si condiciona la frecuencia de complicaciones o reintervención en el conjunto de los recién nacidos y en los grupos antes mencionados.

- Evidenciar si existe relación entre el mismo y el tiempo de ingreso total o el tiempo de ingreso en la Unidad de Cuidados Intensivos Neonatales.

Así mismo pretendemos objetivar si el tiempo total que los pacientes pasan en quirófano puede considerarse también como factor de riesgo de morbimortalidad en los neonatos.

Finalmente nos proponemos diferenciar entre la influencia del tiempo quirúrgico y del tiempo total de quirófano en los pacientes intervenidos en quirófano frente a los operados en la Unidad de Cuidados Intensivos Neonatales (UCIN) para valorar la importancia de la hipotermia como factor de riesgo. 


\section{MATERIAL Y MÉTODOS:}


Hemos realizado un estudio retrospectivo descriptivo de los pacientes recién nacidos intervenidos en el Hospital Materno-Infantil Gregorio Marañón durante el período comprendido entre el 1 de Enero de 2008 y el 31 de Diciembre de 2011 Para ello se realizó, en un primer paso, una base de datos Acces que recogía todas las intervenciones realizadas en dicho hospital desde su inauguración en el año 2003. Se obtuvieron los datos del programa SISCAM del Hospital Gregorio Marañón y se corrigieron aquellos que resultaban erróneos por no coincidir con los existentes en las hojas de intervención quirúrgica, la de Anestesia y la de Enfermería cumplimentadas en el acto quirúrgico y presentes en la historia clínica. En esta base de datos se identifica cada intervención con un número y se registran la fecha y hora de entrada en quirófano, la de inicio de la intervención, la del fin de la misma y la de salida del paciente. También se recogen el nombre de los cirujanos, anestesistas e instrumentistas, el diagnóstico, procedimiento realizado, lugar de la intervención, unidad de procedencia del paciente dentro del hospital y unidad de destino, especialidad del cirujano que practica la intervención y tipo de cirugía según el riesgo de infección así como si el paciente fallece durante la misma.

Dentro de esta base de datos se seleccionan los pacientes intervenidos durante el periodo de tiempo de nuestro estudio.

Finalmente escogemos los diagnósticos propios del período neonatal. Excluimos de este estudio las cirugías neonatales que no precisen habitualmente el ingreso en la Unidad de Cuidados Intensivos Neonatales como las hernias inguinales, la estenosis hipertrófica de píloro, el escroto agudo, endoscopias o cistoscopias. 
Así mismo se excluyen los pacientes tratados por servicios diferentes al de Cirugía Pediátrica. En cuanto al Ductus Arterioso Persistente la cirugía puede llevarse a cabo tanto por cirujanos pediátricos como por cirujanos cardiacos y por ello lo incluimos.

Una vez seleccionados los pacientes de nuestro estudio se reconvierte la base de datos Acces en otra Excel para un mejor manejo de los datos.

Mediante la revisión de historias clínicas e informes de alta se obtiene el resto de información necesaria.

Finalmente se diseña una tabla Excel con las siguientes variables a analizar:

- Numero de paciente: Sirve para identificar a cada paciente del estudio

- Fecha de entrada en quirófano: fecha en la cual el paciente entra en la sala operatoria y comienzan los preparativos de la intervención así como procedimientos anestésicos y de monitorización

- Hora de entrada en quirófano: hora en la cual el paciente entra en la sala operatoria y comienzan los preparativos de la intervención así como procedimientos anestésicos y de monitorización.

- Hora de inicio de la cirugía: hora en la cual se realiza la incisión o comienza el procedimiento quirúrgico propiamente dicho.

- Hora de fin de la cirugía: hora en la cual se da por finalizado el procedimiento quirúrgico que suele coincidir con el fin de la sutura de piel .

- Hora de salida de quirófano: hora en la cual el paciente termina los procedimientos de acondicionamiento y estabilización que permiten su traslado a la unidad de destino, en este caso la Unidad de Cuidados Intensivos Neonatales. 
- Fecha de salida de quirófano: fecha en la cual el paciente termina los procedimientos de acondicionamiento y estabilización que permiten su traslado a la unidad de destino.

- Fecha de nacimiento

- Fecha de ingreso: fecha en la cual el paciente es internado en el Servicio de Neonatología. Puede coincidir con la fecha de nacimiento o no dependiendo de si nace en el hospital o bien ingresa desde Urgencias o a través de un traslado de otro centro.

- Fecha de alta: Momento en el cual el paciente sale del Servicio de Neonatología bien con destino a su domicilio, a otro centro hospitalario o fallece.

- Tiempo de ingreso en UCIN: Número de días que el paciente permanece en la Unidad de Cuidados Intensivos Neonatales.

- Mortalidad: Variable que define si un paciente muere durante el ingreso en el cual se realiza el procedimiento quirúrgico. Se codifica como SI si el paciente fallece y NO si el paciente sobrevive a este primer ingreso

- Complicaciones: Variable que define la existencia de una complicación quirúrgica tras la intervención. En este caso se consideran complicaciones relacionadas exclusivamente con el procedimiento quirúrgico propiamente dicho excluyendo complicaciones médicas generales y las relacionadas con el acto anestésico. Las complicaciones definidas son las siguientes:

- ABSCESO: Acúmulo de pus en lecho quirúrgico hallado por el cirujano en una reintervención por deterioro clínico del paciente. No tiene relación con perforaciones intestinales, 
necrosis intestinal, infección de herida o dehiscencia de anastomosis.

- COARTACIÓN DUCTUS: Disminución del calibre aórtico objetivada por ecocardiografía realizada por cardiólogo pediátrico en el postoperatorio tras ligadura del Ductus Arterioso Persistente y registrado en la historia clínica o informe de alta.

- COMPLICACIONES DE LA HERIDA: Dehiscencia, dehiscencia y supuración, o evisceración- La primera es una separación entre los bordes de la herida quirúrgica que afecta a piel y subcutáneo sin salida de material purulento puesta de manifiesto por el cirujano en la historia clínica o informe de alta del paciente. La segunda es igual a la anterior pero con salida de material purulento. La evisceración consiste en una dehiscencia de todas las capas desde la piel al peritoneo en una laparotomía lo que supone una exposición de las asas intestinales puesta de manifiesto en la historia clínica del paciente por parte del cirujano.

- DEHISCENCIA DE ANASTOMOSIS: discontinuidad en la sutura intestinal o esofágica que produce una fuga de material intestinal o fecaloideo si es en el intestino o saliva si es en el esófago. La primera (intestinal) se documenta como hallazgo quirúrgico puesto de manifiesto por un cirujano en la hoja operatoria de una reintervención. La segunda (esofágica) se documenta por una fuga de saliva a través del drenaje puesta 
de manifiesto por el cirujano en la historia clínica o por la salida de contraste al tórax en el esofagograma de control postoperatorio documentada por el radiólogo en su informe.

- ESTENOSIS: Estrechamiento de la luz intestinal o esofágica que produce una obstrucción al paso del alimento. En este caso nos referiremos exclusivamente a las esofágicas pues las intestinales se codifican como obstrucción intestinal. Se documenta a través del informe escrito por el radiólogo en el esofagograma de control.

- HERNIA INCISIONAL CON OBSTRUCCIÓN INTESTINAL:

Defecto de pared abdominal en la zona de la incisión que atrapa un asa intestinal produciendo una interrupción en el paso del alimento o heces. Se documenta a través del informe quirúrgico de una reintervención escrito por el cirujano.

- NECROSIS INTESTINAL: Pérdida de viabilidad de un segmento intestinal más o menos largo debido a isquemia, inflamación, trauma o infección documentado por la hoja operatoria escrita por el cirujano tras una reintervención o por una evolución letal en pacientes con enterocolitis y afectación del intestino en su totalidad que no responden a una derivación proximal.

- NEUMOTORAX: Acúmulo de aire entre la pared torácica y el pulmón que dificulta la ventilación. Se documenta por informe radiológico de una radiografía de tórax.

- NO: Ausencia de complicaciones 
- OBSTRUCCIÓN INTESTINAL: Interrupción del paso del alimento o heces secundario a una estenosis de la sutura intestinal, infección o bridas postquiúrgicas documentado por un cirujano en la hoja operatoria tras una reintervención.

- PERFORACIÓN INTESTINAL: Alteración en la continuidad de la pared intestinal que produce salida de material intestinal o fecaloideo a la cavidad abdominal y no relacionado con una sutura previa. Documentado por un cirujano en la hoja operatoria de una reintervención.

- REFISTULIZACIÓN: Reaparición de una fístula traqueoesofágica (comunicación entre luz de la tráquea y luz del esófago) previamente tratada durante la cirugía. Documentado por informe radiológico de un esofagograma o por informe quirúrgico escrito por un cirujano tras una broncoscopia diagnóstica.

- SANGRADO: Hemorragia postoperatoria que produce inestabilidad hemodinámica documentada por el intensivista en la historia clínica o es causa de reintervención.

- TRAQUEOMALACIA: Obstrucción dinámica de la luz traqueal tras una cirugía por atresia de esófago que dificulta la extubación del paciente tras el procedimiento y se documenta por el informe quirúrgico de un cirujano tras una broncoscopia diagnóstica.

- Reintervención: Variable que define la necesidad de un paciente de ser operado por segunda vez tras el primer procedimiento. No necesariamente 
la reintervención es secundaria a una complicación. (por ejemplo reconstrucciones del tránsito precoces para mejorar la ganancia ponderal). Se codifica como SI si se precisaron una o más cirugías tras el primer procedimiento y NO si no se produjeron.

- Anestesia: Variable que indica quien anestesió al paciente. Se codifica como ANI si fue un anestesista infantil y como UCIN si fue un intensivista neonatal. En este estudio nos sirve para diferenciar los pacientes que se intervinieron en quirófano (ANI) de los que se operaron en la unidad de cuidados intensivos neonatales (UCIN) y por ello nos referiremos a ella en ocasiones como lugar de intervención.

- Procedimiento: Variable que define el tipo de abordaje que se realizó durante la cirugía para tratar al paciente. Se codifica como:

- ANORRECTOPLASTIA: Abordaje sagital posterior a través del periné para tratar atresias anorrectales de buen pronóstico.

- DRENAJE: Pequeña incisión abdominal para la implantación de un drenaje tipo Penrose en pacientes con enterocolitis demasiado inestables para soportar una laparotomía.

- ECMO: Canalización de arteria carótida y vena yugular interna derechas mediante un abordaje cervical para realizar Oxigenación por Membrana Extracorpórea.

- LAPAROSCOPIA: procedimiento mínimamente invasivo para tratar patologías abdominales que consiste en introducir una óptica en el abdomen a través de una pequeña incisión. Se distiende el abdomen con dióxido de carbono y se introducen pinzas de pequeño tamaño por otras heridas lo que nos 
permite incisiones más pequeñas y una recuperación precoz del paciente.

- MEDICIÓN DE CABOS: Abordaje que combina una endoscopia digestiva alta y un acceso a través de una gastrostomía previa que, bajo control radiológico nos permite definir la distancia entre el esófago proximal y distal en una atresia de esófago.

- TORACOTOMÍA: Abordaje quirúrgico de patología torácica a través de una incisión en el espacio intercostal.

- TORACOTOMÍA Y LAPAROTOMÍA: Combinación de los abordajes antes mencionados en un mismo tiempo quirúrgico.

- Diagnóstico: Variable que indica la enfermedad que fue causa del procedimiento quirúrgico. Los distintos diagnósticos codificados son los siguientes

- Aspiración meconial: Enfermedad que se produce por el paso de una mezcla de líquido amniótico y meconio (heces del recién nacido) hacia la vía aérea y pulmones del neonato provocando un distress respiratorio. No suele requerir tratamiento quirúrgico pero los pacientes más graves pueden beneficiarse de la oxigenación a través de membrana extracorpórea que precisa la canalización de arteria carótida y vena yugular interna con unas cánulas de gran tamaño para permitir la entrada y salida de sangre.

- Atresia anorrectal: Malformación congénita consistente en la ausencia de un ano normoposicionado y de calibre normal en el recién nacido. Es, en realidad un espectro de 
malformaciones que abarcan desde la fístula perineal consistente en la presencia de un ano anterior a su posición habitual y de menor calibre y que tiene un muy buen pronóstico y la cloaca compleja (en niñas) que consiste en la desembocadura del recto, vagina y uretra por un mismo orificio en el periné o la fístula recto-prostática (en varones) con mucho peor pronóstico y que precisa soluciones más complejas. El tratamiento quirúrgico en el recién nacido es una laparotomía y colostomía (en los casos más complejos que precisarán cirugías reconstructivas más adelante) o un abordaje sagital posterior (para la corrección definitiva en los casos más sencillos).

- Atresia anorrectal. Atresia duodenal. Atresia de esófago: Suma de estas 3 malformaciones congénitas.

- Atresia de esófago: Malformación congénita consistente en la interrupción del esófago a nivel del tórax con o sin presencia de una fístula traqueo-esofágica (comunicación patológica entre tráquea y esófago) en el cabo proximal o distal. La más frecuente es la tipo III (con fístula traqueo-esofágica distal) y después la tipo I (sin fístula) y son ambos tipos las que aparecen en este estudio. Se tratan en período neonatal mediante toracotomía, cierre de la fístula y anastomosis termino-terminal del esófago. Si hay mucha distancia entre el cabo proximal y el distal o es una tipo I se realiza una 
laparotomía con gastrostomía y una reconstrucción a largo plazo.

- Atresia de esófago. Atresia anorrectal: Combinación de ambas malformaciones.

- Atresia Duodenal: Interrupción del intestino del recién nacido a nivel del duodeno impidiendo la correcta alimentación. Puede asociarse a páncreas anular. Requiere cirugía en período neonatal consistente en una laparotomía y anastomosis en diamante o laterolateral del duodeno.

- Atresia duodenal. Atresia de vías biliares: Combinación de la malformación anterior con una interrupción de la vía biliar extrahepática. La corrección de esta última requiere una laparotomía y portoenteroanastomosis o intervención de Kasai.

- Ductus Arterioso Persistente: Patología propia del recién nacido prematuro. El ductus arterioso es un remanente de la circulación fetal que comunica la aorta y la arteria pulmonar. Debe cerrarse tras el nacimiento pero no siempre lo hace, sobre todo en prematuros donde puede producir alteraciones hemodinámicas importantes. Puede tratarse medicamente con indometacina o ibuprofeno pero en ocasiones requiere un tratamiento quirúrgico que consiste en la realización de una toracotomía y ligadura del mismo con clips vasculares.

- Defectos de pared abdominal: Se trata en realidad de dos malformaciones diferentes: el onfalocele y la gastrosquisis. En 
el primero existe una falta de cierre de la pared abdominal a nivel del ombligo y el intestino no está expuesto al exterior al estar cubierto por una membrana. Aun así tiene peor pronóstico que la gastrosquisis al asociarse con frecuencia a malformaciones cardiacas. La gastrosquisis es un defecto de la pared abdominal localizado a la derecha del ombligo. Las asas están expuestas al exterior y dañadas por el meconio por lo que se cubren por una capa inflamatoria llamada "peel". Puede asociarse a atresias intestinales y es causa de intestino corto en muchos pacientes. El tratamiento de ambas malformaciones puede realizarse en un tiempo(cierre directo) o en dos (Silo). Ambas son cirugías abdominales y podrían considerarse como laparotomías.

- Enfisema Lobar Congénito: Malformación congénita que produce una hiperinsuflación del lóbulo pulmonar afecto dando lugar a una burbuja de aire que desplaza el tejido pulmonar sano. Su tratamiento puede ser una urgencia quirúrgica y consiste en una toracotomía y lobectomía pulmonar del lóbulo pulmonar comprometido.

- Enterocolitis necrotizante: Enfermedad inflamatoria-infecciosa del intestino que puede producir necrosis y perforación del mismo. Es propia del recién nacido pretérmino. Su tratamiento inicial es conservador pero puede precisar cirugía ante una afectación extensa del intestino o perforación. Suele tratarse mediante laparotomía, resección intestinal y enterostomía. En 
caso de inestabilidad hemodinámica que impida al paciente sobrevivir a una intervención tan cruenta puede tratarse mediante drenaje abdominal y laparotomía diferida cuando mejore clínicamente.

- Hernia diafragmática congénita: Malformación congénita consistente en una solución de continuidad en el diafragma. Existen varios tipos, el más frecuente en neonatos es la hernia diafragmática posterolateral o de Bochdalek. Su pronóstico depende del grado de hipoplasia pulmonar que asocie. La cirugía consiste en una laparotomía y cierre del defecto con sutura simple o con parches .

- Obstrucción intestinal neonatal: En esta opción se han codificado todas aquellas causas que produzcan una dificultad del paso del alimento o las heces a través de la luz intestinal más allá del duodeno y antes del ano. Puede ser causada por atresias intestinales (interrupción de la luz intestinal secundaria a episodios de isquemia durante el embarazo), malrotación intestinal ( ausencia de rotación o rotación incompleta del intestino durante su desarrollo embrionario), ileo meconial (obstrucción intraluminal provocada por un meconio demasiado espeso), enfermedad de Hirschsprung (Ausencia de neuronas en los plexos de la pared intestinal del colon que produce una obstrucción funcional). Todas estas enfermedades se tratan mediante una laparotomía pudiendo requerir resección intestinal o no. 


\section{La tabla definitiva obtenida se adjunta en las últimas páginas de esta tesis}

(TABLA XI)

\section{CRITERIOS DE INCLUSIÓN}

- Paciente ingresado en la Unidad de Neonatología

- Intervenido en el período de tiempo desde el 1 de Enero de 2008 al 31 de diciembre de 2011 en el Hospital Infantil Gregorio Marañón

- Sometido a un procedimiento quirúrgico que precise ingreso en la Unidad de Cuidados Intensivos Neonatales.

- El procedimiento debe ser llevado a cabo habitualmente por un cirujano pediátrico

\section{CRITERIOS DE EXCLUSIÓN}

- Recién nacidos que no ingresen en la unidad de neonatología (Ejemplo: Estenosis Hipertrófica de Píloro)

- Neonatos intervenidos que no ingresen en la Unidad de Cuidados Intensivos Neonatales (Ejemplo: Hernias inguinales)

- Pacientes intervenidos fuera del periodo de tiempo antes mencionado

- Neonatos cuya intervención se lleve a cabo habitualmente por profesionales diferentes al cirujano pediátrico. (Cirugías de Oftalmología, Otorrinolaringología, Neurocirugía, Traumatología y Ortopedia, Cirugía Maxilofacial o Cirugía Cardiaca, a excepción del Ductus Arterioso Persistente que puede llevarse a cabo tanto por cirujanos cardiacos como cirujanos pediátricos)

- Pacientes en los que no se haya podido completar la recogida de todos las variables de la tabla antes mencionada. 
El procedimiento quirúrgico sobre el cual se analizarán las distintas variables será el primero al cual se sometió el paciente en el caso de que este precise varias intervenciones.

\section{MÉTODO ESTADÍSTICO}

Hemos resumido las variables numéricas con medias, desviaciones típicas y percentiles y las variables cualitativas y las variables resultantes de categorizar las variables numéricas con porcentajes.

Hemos resumido en tablas de contingencia las relaciones entre parejas de variables.

Para contrastar si la distribución de las variables que representan tiempo (edad, días de estancia, tiempo de cirugía y de quirófano, tiempo UCI) era la misma en las tipologías determinadas por variables SI/NO utilizamos el test de Wilcoxon-MannWhitney y para contrastar la noexistencia relación entre las variables numéricas utilizamos el test derivado del coeficiente de correlación de Spearman. Consideramos como estadísticamente significativos valores de p inferiores a 0.05 . Los datos se analizaron utilizando el paquete estadístico SAS versión 9.2. (SAS Institute Inc., Cary, NC, USA. URL http://www.sas.com) y el paquete estadístico R. versión 3.2.1 ( R Core Team (2013). R: A language and environment for statistical computing. R Foundation for Statistical Computing, Vienna, Austria. URL http://www.R-project.org) Una vez obtenida la tabla realizamos los estudios estadísticos. Para ello se usaron los programas SAS v9.2. SAS Institute Inc., Cary, NC, USA.

Paquete estadístico R. versión 3.2.1. 
RESULTADOS 


\section{NÚMERO DE PACIENTES:}

El número total de intervenciones realizadas en el Hospital Infantil Gregorio Marañón entre el 1 de Enero de 2008 y el 31 de Diciembre de 2011 fue de 15366.

De dichos pacientes 202 cumplieron los criterios de inclusión iniciales de este estudio, sin embargo en 8 pacientes no se pudieron codificar todos los datos de la tabla por no estar presentes en la historia clínica por lo que el número final de pacientes incluidos en el estudio fue de 194.

\section{EDAD:}

La media de edad en el momento de la cirugía fue de 16.28 días. En la siguiente tabla se muestra la media, desviación típica y percentiles de la edad:

$\begin{array}{rrccccccccc}\text { Media } & \text { Dev tip } & \text { Mínimo } & \text { Pc5 } & \text { Pc10 } & \text { Pr25 } & \text { Pc50 } & \text { Pr75 } & \text { Pc90 } & \text { Pc95 } & \text { Máximo } \\ 16.28 & 27.85 & 0.00 & 0.00 & 0.00 & 1.00 & 10.00 & 22.00 & 41.00 & 52.00 & 328.00\end{array}$

TABLA XII: MEDIA Y PERCENTILES DE LA EDAD

\section{TIEMPO QUIRÚRGICO:}

El tiempo quirúrgico medio fue de 1.36 horas . En la siguiente tabla se muestra la distribución del tiempo quirúrgico por intervalos medido en horas:

\begin{tabular}{|lcc|}
\hline cir_horas_ & Frecuencia & Porcentaje \\
de00 a 0.5 & 31 & 15.98 \\
de00.5a01 & 45 & 23.20 \\
de01 a 02 & 83 & 42.78 \\
de02 a 03 & 23 & 11.86 \\
de03a99 & 12 & 6.19 \\
\hline
\end{tabular}

TABLA XIII: DISTRIBUCIÓN DEL TIEMPO QUIRÚRGICO EN INTERVALOS 


\section{TIEMPO TOTAL DE QUIRÓFANO:}

El tiempo total de quirófano medio fue de 2.63 horas. En la siguiente tabla se muestra la distribución del tiempo total de quirófano por intervalos medido en horas:

\begin{tabular}{|lcc|}
\hline $\begin{array}{l}\text { quir_ } \\
\text { horas_ }\end{array}$ & Frecuencia & Porcentaje \\
\hline de00a01 & 14 & 7.22 \\
de01a02 & 57 & 29.38 \\
de02a03 & 51 & 26.29 \\
de03a04 & 42 & 21.65 \\
de04a99 & 30 & 15.46 \\
\hline
\end{tabular}

TABLA XIV: DISTRIBUCIÓN DEL TIEMPO TOTAL DE QUIRÓFANO POR INTERVALOS

\section{TIEMPO DE INGRESO:}

El tiempo de ingreso medio fue de 71.16 días. En la siguiente tabla se muestra la distribución del tiempo de ingreso por intervalos medido en días:

\begin{tabular}{|lrc|}
\hline estancia_ & Frecuencia & Porcentaje \\
\hline de000a007 & 8 & 4.12 \\
de008a014 & 23 & 11.86 \\
de015a030 & 41 & 21.13 \\
de031a060 & 33 & 17.01 \\
de061a120 & 54 & 27.84 \\
de121a999 & 35 & 18.04 \\
\hline
\end{tabular}

TABLA XV: DISTRIBUCIÓN DEL TIEMPO DE INGRESO EN INTERVALOS 


\section{TIEMPO DE INGRESO EN UCIN:}

El tiempo medio de ingreso en UCIN fue de 31.24 días. En la siguiente tabla se muestra la distribución del tiempo de ingreso en UCIN por intervalos medido en días:

\begin{tabular}{|lcc|}
\hline UCIN__ & Frecuencia & Porcentaje \\
\hline de00a01 & 21 & 10.82 \\
de02a03 & 18 & 9.28 \\
de04a07 & 22 & 11.34 \\
de07a14 & 34 & 17.53 \\
de15a30 & 36 & 18.56 \\
de31a99 & 63 & 32.47 \\
\hline
\end{tabular}

TABLA XVI: DISTRIBUCIÓN DEL TIEMPO DE INGRESO EN UCIN POR INTERVALOS

Todas estas variables numéricas con sus medias, desviaciones típicas y percentiles se muestran en la siguiente tabla:

\begin{tabular}{|l|l|r|r|r|r|r|r|r|r|r|r|r|r|}
\hline Variable & Etiqueta & $\mathrm{N}$ & \multicolumn{1}{|l|}{ Media } & Devtip & Mínimo & P5 & \multicolumn{1}{l|}{ p10 } & p25 & p50 & p75 & \multicolumn{1}{l|}{ p90 } & p95 & maximo \\
\hline quir_horas & quir_horas & 194 & 2,63 & 1,28 & 0,42 & 0,92 & 1,08 & 1,58 & 2,48 & 3,33 & 4,25 & 5,25 & 6,42 \\
\hline cir_horas & cir_horas & 194 & 1,36 & 0,87 & 0,17 & 0,33 & 0,42 & 0,67 & 1,17 & 1,75 & 2,25 & 3,08 & 4,92 \\
\hline estancia & estancia & 194 & 71,16 & 70,3 & 1 & 8 & 12 & 19 & 47,5 & 107 & 156 & 189 & 660 \\
\hline T._UCIN - & T. UCIN & 194 & 31,24 & 55,75 & 0 & 1 & 1 & 5 & 15 & 41 & 64 & 101 & 630 \\
\hline
\end{tabular}

TABLA XVII: VARIABLES NUMÉRICAS ANALIZADAS

\section{MORTALIDAD:}

La mortalidad fue del $19.07 \%$ como se muestra en la tabla siguiente:

\begin{tabular}{|rrr|}
\hline \multicolumn{3}{c}{ MORTALIDAD } \\
MORTALIDAD & Frecuencia & Porcentaje \\
\hline 0 & 157 & 80.93 \\
1 & 37 & 19.07 \\
\hline
\end{tabular}

TABLA XVIII: MORTALIDAD 


\section{COMPLICACIONES:}

Un 30.41\% de los pacientes presentaron alguna complicación quirúrgica.

En las siguientes tablas aparecen el numero de complicaciones y su frecuencia:

\begin{tabular}{|lrc|}
\hline complicaciones_ & Frecuencia & Porcentaje \\
\hline NO & 135 & 69.59 \\
SI & 59 & 30.41 \\
\hline
\end{tabular}

TABLA XIXa: COMPLICACIONES TOTALES

\begin{tabular}{lrc|}
\hline complicaciones_ & Frecuencia & Porcentaje \\
\hline ABSCESO & 1 & 0.52 \\
COARTACION DUCTUS & 1 & 0.52 \\
COMPL HERIDA & 11 & 5.67 \\
DEHISCENCIA ANASTOMOSIS & 5 & 2.58 \\
ESTENOSIS & 3 & 1.55 \\
HERNIA INCISIONAL Y OBSTRUCCION INTE & 1 & 0.52 \\
NECROSIS INTESTINAL & 18 & 9.28 \\
NEUMOTORAX & 1 & 0.52 \\
NO & 136 & 70.10 \\
OBSTRUCCION INTESTINAL & 9 & 4.64 \\
PERFORACION INTESTINAL & 3 & 1.55 \\
REFISULIZACION & 1 & 0.52 \\
SANGRADO & 3 & 1.55 \\
TRAQUEOMALACIA & 1 & 0.52 \\
\hline \hline
\end{tabular}

TABLA XIXb: COMPLICACIONES ESPECÍFICAS

\section{REINTERVENCIÓN:}

El 32.47\% de los pacientes intervenidos precisó al menos una reintervención durante su ingreso en Neonatología como se muestra en la siguiente tabla:

\begin{tabular}{|crc|}
\hline \multicolumn{3}{|c|}{ REINTERVENCION } \\
REINTERVENCION & Frecuencia & Porcentaje \\
NO & 131 & 67.53 \\
SI & 63 & 32.47 \\
\hline
\end{tabular}

TABLA XX: REINTERVENCIONES 


\section{LUGAR DE LA CIRUGÍA (ANESTESIA):}

El 24.74\% de los pacientes fue intervenido en la Unidad de Cuidados Intensivos Neonatales (UCIN), el 75.26 \% restante en quirófano (ANI) como se muestra en la tabla:

\begin{tabular}{|lrc|}
\hline \multicolumn{3}{c|}{ ANESTESIA } \\
ANESTESIA & Frecuencia & Porcentaje \\
\hline ANI & 146 & 75.26 \\
UCIN & 48 & 24.74 \\
\hline
\end{tabular}

TABLA XXI: LUGAR DE LA INTERVENCIÓN (ANESTESIA)

\section{DIAGNÓSTICO:}

En la siguiente tabla se muestra la frecuencia de diagnósticos quirúrgicos. Los más frecuentes son la Enterocolitis Necrotizante (22.16\%) y el Ductus Arterioso Persistente (20.62\%) .

\begin{tabular}{lrr} 
diagnostico_ & Frecuencia & Porcentaje \\
\hline ASPIRACION MECONIAL & 5 & 2.58 \\
ATRESIA ANORRECTAL & 10 & 5.15 \\
ATRESIA ANORRECTAL, DUODENAL Y ESOFAGO & 1 & 0.52 \\
ATRESIA DE ESOFAGO & 18 & 9.28 \\
ATRESIA DE ESOFAGO. ATRESIA ANO-RECTAL & 1 & 0.52 \\
ATRESIA DUODENAL & 14 & 7.22 \\
ATRESIA DUODENAL Y ATRESIA DE VIAS BILIAR & 1 & 0.52 \\
CIERRE DUCTUS & 40 & 20.62 \\
DEFECTOS DE PARED ABDOM & 14 & 7.22 \\
ENFISEMA LOBAR CONGENITO & 1 & 0.52 \\
ENTEROCOLITIS & 43 & 22.16 \\
HERNIA DIAFRAGMATICA & 20 & 10.31 \\
OBSTR INTESTINAL & 26 & 13.40
\end{tabular}

TABLA XXII: DIAGNÓSTICOS 


\section{PROCEDIMIENTO:}

En la siguiente tabla se muestran las frecuencias de los procedimientos quirúrgicos, siendo los más frecuentes la laparotomía (62.89\%) y la toracotomía (26.8\%):

\begin{tabular}{|lrc|}
\hline procedimiento_ & Frecuencia & Porcentaje \\
\hline ANORRECTOPLASTIA & 4 & 2.06 \\
ECMO & 5 & 2.58 \\
LAPAROSCOPIA & 7 & 3.61 \\
LAPAROTOMIA & 122 & 62.89 \\
MEDICIÓN DE CABOS & 2 & 1.03 \\
TORACOTOMIA & 52 & 26.80 \\
TORACOTOMIA Y LAPAROTOMIA & 2 & 1.03 \\
\hline
\end{tabular}

TABLA XXIII: PROCEDIMIENTOS

\section{RELACIÓN ENTRE TIEMPO QUIRÚRGICO Y MORTALIDAD:}

En la siguiente tabla se muestra la relación entre tiempo quirúrgico y mortalidad:

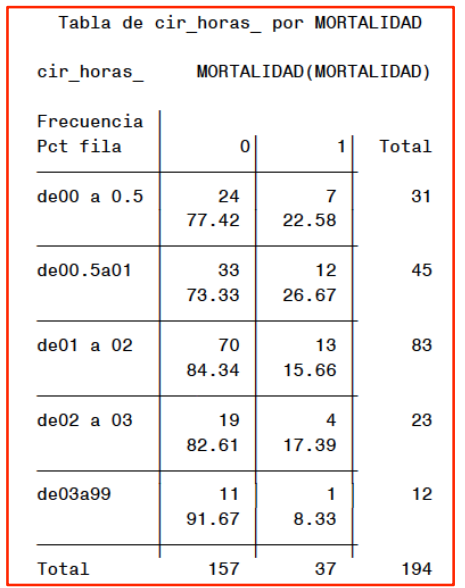

TABLA XXIV: TIEMPO QUIRÚRGICO/MORTALIDAD

No existe relación estadísticamente significativa entre ambas. $P=0.199$ 


\section{RELACIÓN ENTRE TIEMPO TOTAL DE QUIRÓFANO Y MORTALIDAD:}

En la siguiente tabla y gráfico se muestra la relación entre tiempo total de quirófano y mortalidad:

\begin{tabular}{|c|c|c|c|}
\hline \multicolumn{4}{|c|}{$\begin{array}{l}\text { Tabla de quir_horas_por MORTALIDAD } \\
\text { quir_horas__} \\
{ }_{\text {MORTALIDAD (MORTALIDAD) }}\end{array}$} \\
\hline $\begin{array}{l}\text { Frecuencia } \\
\text { Pct fila }\end{array}$ & 0 & 1 & Total \\
\hline de00a01 & $\begin{array}{r}9 \\
64.29\end{array}$ & $\begin{array}{r}5 \\
35.71\end{array}$ & 14 \\
\hline de01a02 & $\begin{array}{r}46 \\
80.70\end{array}$ & $\begin{array}{r}11 \\
19.30\end{array}$ & 57 \\
\hline de02a03 & $\begin{array}{r}42 \\
82.35\end{array}$ & $\begin{array}{r}9 \\
17.65\end{array}$ & 51 \\
\hline de03a04 & $\begin{array}{r}32 \\
76.19\end{array}$ & $\begin{array}{r}10 \\
23.81\end{array}$ & 42 \\
\hline de04a99 & $\begin{array}{r}28 \\
93.33\end{array}$ & $\begin{array}{r}2 \\
6.67\end{array}$ & 30 \\
\hline Total & 157 & 37 & 194 \\
\hline
\end{tabular}

TABLA XXV: TIEMPO TOTAL DE QUIRÓFANO/ MORTALIDAD

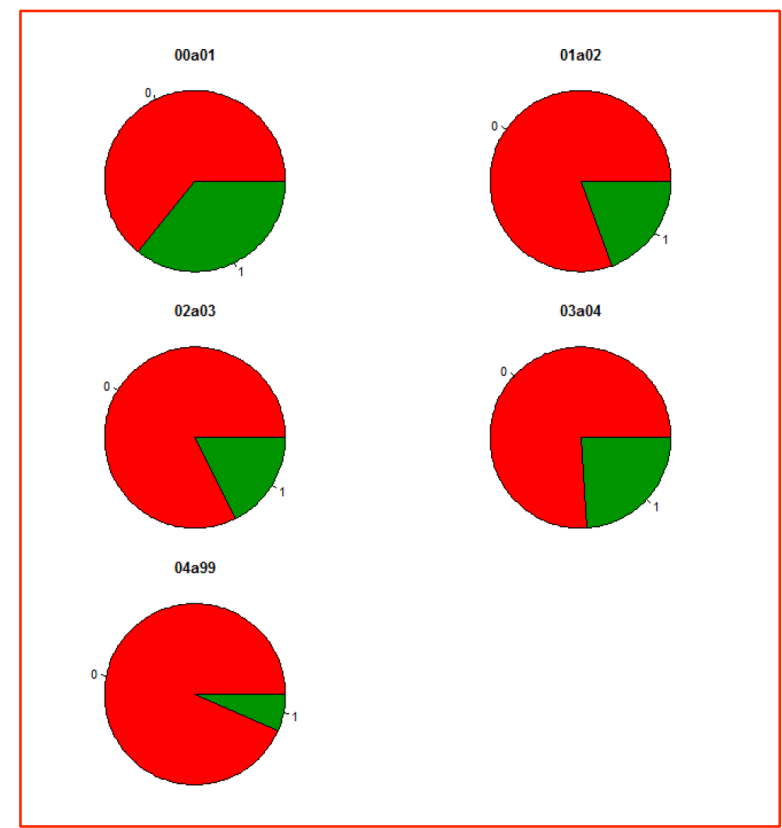

GRÁFICO I: RELACIÓN ENTRE TIEMPO TOTAL DE QUIRÓFANO Y MORTALIDAD

No existe relación estadísticamente significativa entre ambas $P=0.0733$ 


\section{RELACIÓN ENTRE TIEMPO QUIRÚRGICO Y TIEMPO DE INGRESO:}

En la siguiente tabla y gráfico se muestra la relación entre tiempo quirúrgico y tiempo de ingreso:

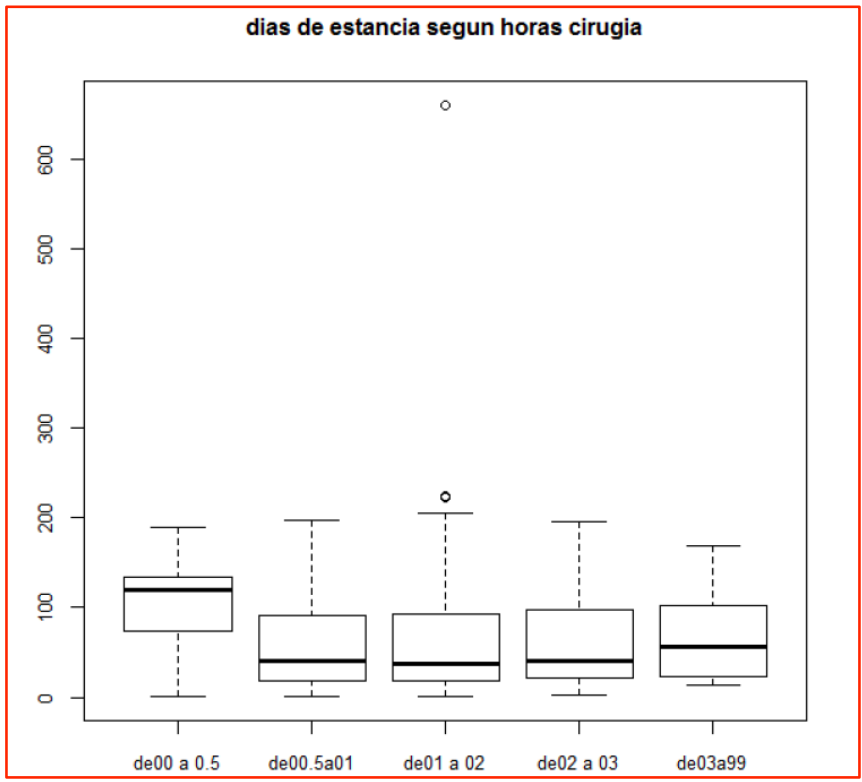

GRÁFICO II: TIEMPO QUIRÚRGICO/TIEMPO DE INGRESO

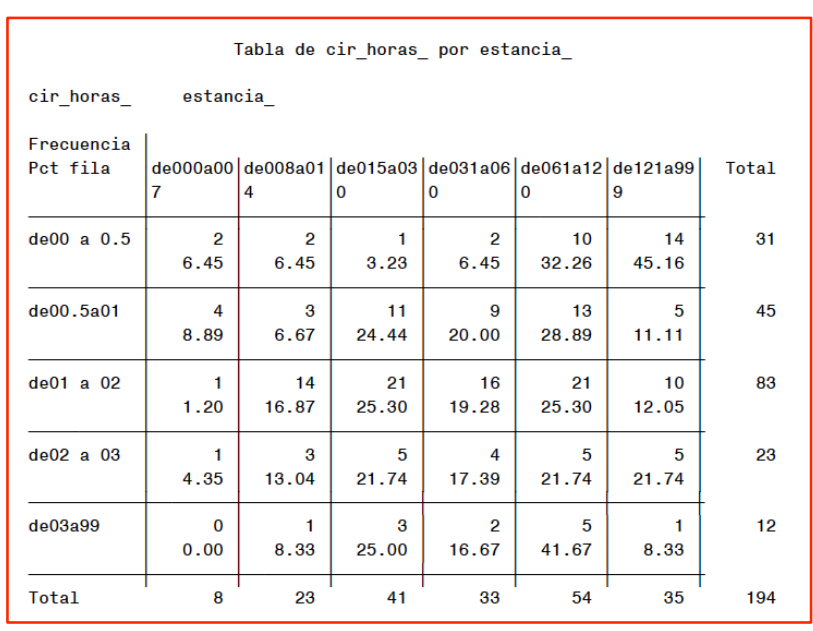

TABLA XXVI: TIEMPO QUIRÚRGICO/ TIEMPO DE INGRESO

No existe relación estadísticamente significativa entre ambas $P=0.1258$ 
RELACIÓN ENTRE TIEMPO TOTAL DE QUIRÓFANO Y TIEMPO DE INGRESO:

En la siguiente tabla y gráfico se muestra la relación entre tiempo total de quirófano y tiempo de ingreso:

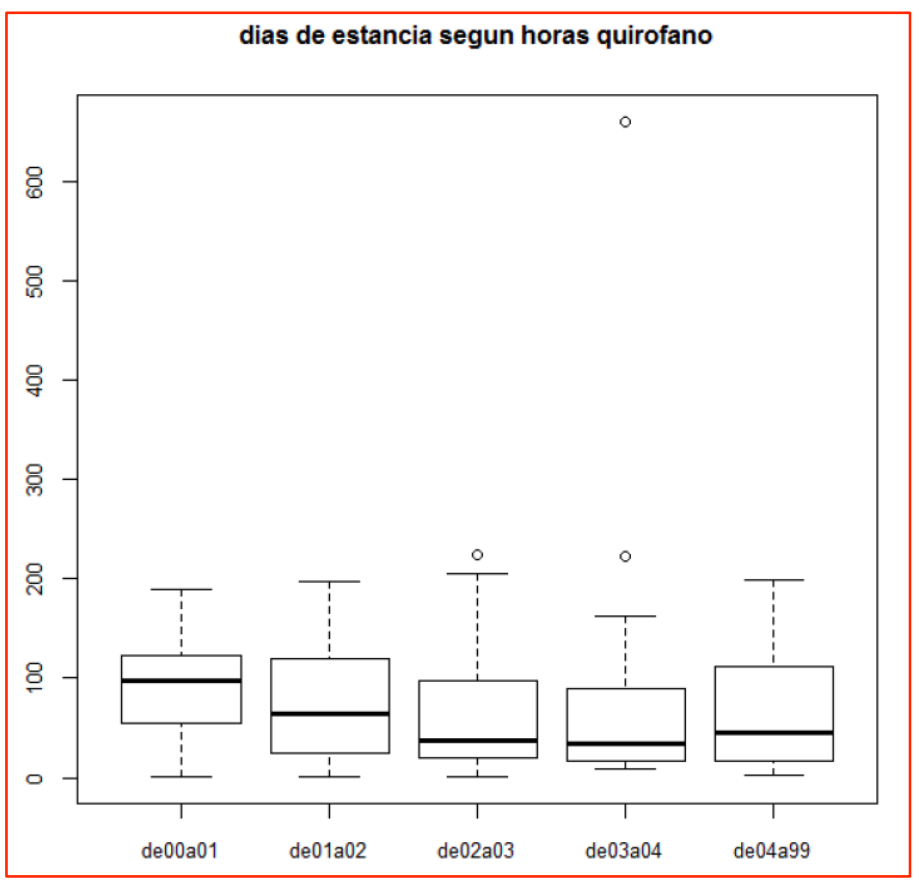

GRÁFICO III: TIEMPO TOTAL DE QUIRÓFANO/ TIEMPO DE INGRESO

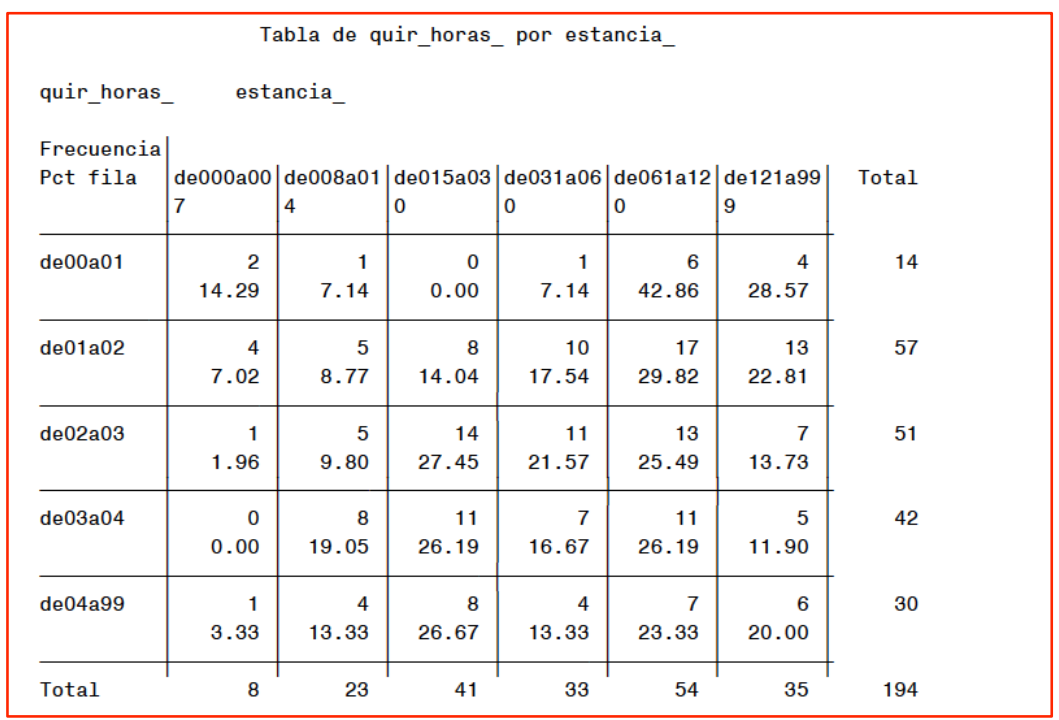

TABLA XXVII: TIEMPO TUTAL DE QUIRÓFANO/ TIEMPO DE INGRESO

No existe relación estadísticamente significativa entre ambas $\mathrm{P}=0.0605$ 
RELACIÓN ENTRE TIEMPO QUIRÚRGICO Y TIEMPO DE INGRESO EN UCIN:

En la siguiente tabla se muestra la relación entre tiempo quirúrgico y tiempo de ingreso en UCIN:

\begin{tabular}{|c|c|c|c|c|c|c|c|}
\hline \multicolumn{8}{|c|}{ Tabla de cir_horas_por TIEMPO_UCIN_ } \\
\hline $\begin{array}{l}\text { Frecuencia } \\
\text { Pct fila }\end{array}$ & de00a01 & de02a03 & de04a07 & de07a14 & de15a30 & de31a99 & Total \\
\hline de00 a 0.5 & $\begin{array}{r}2 \\
6.45\end{array}$ & $\begin{array}{r}0 \\
0.00\end{array}$ & $\begin{array}{r}3 \\
9.68\end{array}$ & $\begin{array}{r}2 \\
6.45\end{array}$ & $\begin{array}{r}4 \\
12.90\end{array}$ & $\begin{array}{r}20 \\
64.52\end{array}$ & 31 \\
\hline de00.5a01 & $\begin{array}{r}6 \\
13.33\end{array}$ & $\begin{array}{r}5 \\
11.11\end{array}$ & $\begin{array}{r}3 \\
6.67\end{array}$ & $\begin{array}{r}6 \\
13.33\end{array}$ & $\begin{array}{r}9 \\
20.00\end{array}$ & $\begin{array}{r}16 \\
35.56\end{array}$ & 45 \\
\hline de01 a 02 & $\begin{array}{r}9 \\
10.84\end{array}$ & $\begin{array}{r}11 \\
13.25\end{array}$ & $\begin{array}{r}12 \\
14.46\end{array}$ & $\begin{array}{r}17 \\
20.48\end{array}$ & $\begin{array}{r}16 \\
19.28\end{array}$ & $\begin{array}{r}18 \\
21.69\end{array}$ & 83 \\
\hline de02 a 03 & $\begin{array}{r}2 \\
8.70\end{array}$ & $\begin{array}{r}2 \\
8.70\end{array}$ & $\begin{array}{r}2 \\
8.70\end{array}$ & $\begin{array}{r}6 \\
26.09\end{array}$ & $\begin{array}{r}5 \\
21.74\end{array}$ & $\begin{array}{r}6 \\
26.09\end{array}$ & 23 \\
\hline de03a99 & $\begin{array}{r}2 \\
16.67\end{array}$ & $\begin{array}{r}0 \\
0.00\end{array}$ & $\begin{array}{r}2 \\
16.67\end{array}$ & $\begin{array}{r}3 \\
25.00\end{array}$ & $\begin{array}{r}2 \\
16.67\end{array}$ & $\begin{array}{r}3 \\
25.00\end{array}$ & 12 \\
\hline Total & 21 & 18 & 22 & 34 & 36 & 63 & 194 \\
\hline
\end{tabular}

TABLA XXVIII: TIEMPO QUIRÚRGICO/TIEMPO DE INGRESO EN UCIN

Existe relación estadísticamente significativa entre ambos $\mathrm{P}=\mathbf{0 . 0 1 1 1}$ $(\mathrm{P}<\mathbf{0 . 0 5})$ 
RELACIÓN ENTRE TIEMPO TOTAL DE QUIRÓFANO Y TIEMPO DE INGRESO

\section{EN UCIN:}

En la siguiente tabla se muestra la relación entre tiempo total de quirófano y tiempo de ingreso en UCIN:

\begin{tabular}{|c|c|c|c|c|c|c|c|}
\hline \multicolumn{8}{|c|}{ Tabla de quir_horas_por TIEMPO_UCIN_ } \\
\hline $\begin{array}{l}\text { Frecuencia } \\
\text { Pct fila }\end{array}$ & de00a01 & de02a03 & de04a07 & de07a14 & de15a30 & de31a99 & Total \\
\hline de00a01 & $\begin{array}{r}1 \\
7.14\end{array}$ & $\begin{array}{r}0 \\
0.00\end{array}$ & $\begin{array}{r}0 \\
0.00\end{array}$ & $\begin{array}{r}2 \\
14.29\end{array}$ & $\begin{array}{r}2 \\
14.29\end{array}$ & $\begin{array}{r}9 \\
64.29\end{array}$ & 14 \\
\hline de01a02 & $\begin{array}{r}5 \\
8.77\end{array}$ & $\begin{array}{r}4 \\
7.02\end{array}$ & $\begin{array}{r}4 \\
7.02\end{array}$ & $\begin{array}{r}8 \\
14.04\end{array}$ & $\begin{array}{r}12 \\
21.05\end{array}$ & $\begin{array}{r}24 \\
42.11\end{array}$ & 57 \\
\hline de02a03 & $\begin{array}{r}8 \\
15.69\end{array}$ & $\begin{array}{r}6 \\
11.76\end{array}$ & $\begin{array}{r}6 \\
11.76\end{array}$ & $\begin{array}{r}8 \\
15.69\end{array}$ & $\begin{array}{r}7 \\
13.73\end{array}$ & $\begin{array}{r}16 \\
31.37\end{array}$ & 51 \\
\hline de03a04 & $\begin{array}{r}3 \\
7.14\end{array}$ & $\begin{array}{r}5 \\
11.90\end{array}$ & $\begin{array}{r}8 \\
19.05\end{array}$ & $\begin{array}{r}8 \\
19.05\end{array}$ & $\begin{array}{r}11 \\
26.19\end{array}$ & $\begin{array}{r}7 \\
16.67\end{array}$ & 42 \\
\hline de04a99 & $\begin{array}{r}4 \\
13.33\end{array}$ & $\begin{array}{r}3 \\
10.00\end{array}$ & $\begin{array}{r}4 \\
13.33\end{array}$ & $\begin{array}{r}8 \\
26.67\end{array}$ & $\begin{array}{r}4 \\
13.33\end{array}$ & $\begin{array}{r}7 \\
23.33\end{array}$ & 30 \\
\hline Total & 21 & 18 & 22 & 34 & 36 & 63 & 194 \\
\hline
\end{tabular}

TABLA XXIX: TIEMPO TOTAL DE QUIRÓFANO/ TIEMPO DE INGRESO EN UCIN

Existe relación estadísticamente significativa entre ambos $\mathbf{P}=0.0002$

$(\mathrm{P}<0.05)$ 


\section{RELACIÓN ENTRE TIEMPO QUIRÚRGICO Y COMPLICACIONES:}

En la siguiente tabla se muestra la relación entre tiempo quirúrgico y las complicaciones postoperatorias:

\begin{tabular}{|c|c|c|c|}
\hline \multicolumn{4}{|c|}{$\begin{array}{l}\text { Tabla de cir_horas_por complica } \\
\text { cir_horas_ complicaciones }\end{array}$} \\
\hline $\begin{array}{l}\text { Frecuencia } \\
\text { Pct fila }\end{array}$ & NO & SI & Total \\
\hline de00 a 0.5 & $\begin{array}{r}27 \\
87.10\end{array}$ & $\begin{array}{r}4 \\
12.90\end{array}$ & 31 \\
\hline de00.5a01 & $\begin{array}{r}32 \\
71.11\end{array}$ & $\begin{array}{r}13 \\
28.89\end{array}$ & 45 \\
\hline de01 a 02 & $\begin{array}{r}55 \\
66.27\end{array}$ & $\begin{array}{r}28 \\
33.73\end{array}$ & 83 \\
\hline de02 a 03 & $\begin{array}{r}16 \\
69.57\end{array}$ & $\begin{array}{r}7 \\
30.43\end{array}$ & 23 \\
\hline de03a99 & $\begin{array}{r}5 \\
41.67\end{array}$ & $\begin{array}{r}7 \\
58.33\end{array}$ & 12 \\
\hline Total & 135 & 59 & 194 \\
\hline
\end{tabular}

TABLA XXX TIEMPO QUIRÚRGICO/COMPLICACIONES

Existe una relación estadísticamente significativa entre ambos $P=0.0121$ $(\mathrm{P}<0.05)$ 
RELACIÓN ENTRE TIEMPO TOTAL DE QUIRÓFANO Y COMPLICACIONES:

En la siguiente tabla y gráfico se muestra la relación entre tiempo total de quirófano y las complicaciones postoperatorias:

\begin{tabular}{|c|c|c|c|}
\hline \multicolumn{4}{|c|}{$\begin{array}{c}\text { abla de quir_horas_ por complicacio } \\
\text { quir_horas__ complicaciones__ }\end{array}$} \\
\hline \multicolumn{4}{|c|}{ Frecuencia| } \\
\hline Pct fila & NO & SI & Total \\
\hline de00a01 & 9 & 5 & 14 \\
\hline & & & \\
\hline de01a02 & 45 & 12 & 57 \\
\hline & 78.95 & 21.05 & \\
\hline de02a03 & 37 & 14 & 51 \\
\hline & 72.55 & 27.45 & \\
\hline de03a04 & 28 & 14 & 42 \\
\hline & 66.67 & 33.33 & \\
\hline de04a99 & 16 & & 30 \\
\hline & 53.33 & 46.67 & \\
\hline Total & 135 & 59 & 194 \\
\hline
\end{tabular}

TABLA XXXI TIEMPO TOTAL DE QUIRÓFANO/ COMPLICACIONES

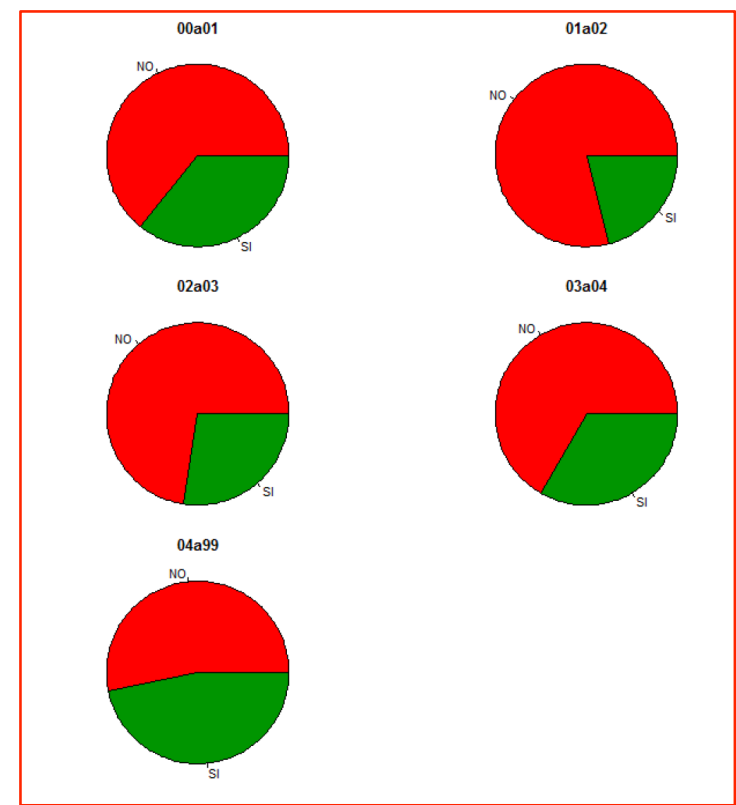

GRAFICO IV: RELACIÓN ENTRE TIEMPO TOTAL DE QUIRÓFANO Y COMPLICACIONES

Existe relación estadísticamente significativa entre ambos $P=0,0343$ $(\mathrm{P}<0.05)$ 


\section{RELACIÓN ENTRE TIEMPO QUIRÚRGICO Y REINTERVENCIÓN:}

En la siguiente tabla se muestra la relación entre tiempo quirúrgico y reintervención:

\begin{tabular}{|c|c|c|c|}
\hline \multicolumn{4}{|c|}{$\begin{array}{l}\text { Tabla de cir_horas_por REINTERVENCION } \\
\text { cir_horas__ }\end{array}$} \\
\hline $\begin{array}{l}\text { Frecuencia } \\
\text { Pct fila }\end{array}$ & No & |SI & Total \\
\hline de00 a 0.5 & $\begin{array}{r}29 \\
93.55\end{array}$ & $\begin{array}{r}2 \\
6.45\end{array}$ & 31 \\
\hline de $00.5 \mathrm{a} 01$ & $\begin{array}{r}28 \\
62.22\end{array}$ & $\begin{array}{r}17 \\
37.78\end{array}$ & 45 \\
\hline de01 a 02 & $\begin{array}{r}53 \\
63.86\end{array}$ & $\begin{array}{r}30 \\
36.14\end{array}$ & 83 \\
\hline de02 a 03 & $\begin{array}{r}15 \\
65.22\end{array}$ & $\begin{array}{r}8 \\
34.78\end{array}$ & 23 \\
\hline de03a99 & $\begin{array}{r}6 \\
50.00\end{array}$ & $\begin{array}{r}6 \\
50.00\end{array}$ & 12 \\
\hline Total & 131 & 63 & 194 \\
\hline
\end{tabular}

TABLA XXXII: TIEMPO QUIRÚRGICO/REINTERVENCIÓN

Existe relación estadísticamente significativa entre ambos $\mathrm{P}=\mathbf{0 . 0 0 3 5}$ $(\mathrm{P}<0.05)$ 


\section{RELACIÓN ENTRE TIEMPO TOTAL DE QUIRÓFANO Y REINTERVENCIÓN:}

En la siguiente tabla se muestra la relación entre tiempo total de quirófano y reintervención:

\begin{tabular}{|c|c|c|c|}
\hline \multicolumn{4}{|c|}{$\begin{array}{l}\text { Tabla de quir_horas_por REINTERVENCION } \\
\text { quir_horas_ } \\
\text { REINTERVENCION (REINTERVENCION) }\end{array}$} \\
\hline $\begin{array}{l}\text { Frecuencia } \\
\text { Pct fila }\end{array}$ & No & |SI & Total \\
\hline de00a01 & $\begin{array}{r}12 \\
85.71\end{array}$ & $\begin{array}{r}2 \\
14.29\end{array}$ & 14 \\
\hline de01a02 & $\begin{array}{r}43 \\
75.44\end{array}$ & $\begin{array}{r}14 \\
24.56\end{array}$ & 57 \\
\hline de02a03 & $\begin{array}{r}31 \\
60.78\end{array}$ & $\begin{array}{r}20 \\
39.22\end{array}$ & 51 \\
\hline de03a04 & $\begin{array}{r}27 \\
64.29\end{array}$ & $\begin{array}{r}15 \\
35.71\end{array}$ & 42 \\
\hline de04a99 & $\begin{array}{r}18 \\
60.00\end{array}$ & $\begin{array}{r}12 \\
40.00\end{array}$ & 30 \\
\hline Total & 131 & 63 & 194 \\
\hline
\end{tabular}

TABLA XXXIII: RELACIÓN ENTRE TIEMPO TOTAL DE QUIRÓFANO Y REINTERVENCIÓN

Existe relación estadísticamente significativa entre ambos $\mathrm{P}=\mathbf{0 . 0 2 6 9}$ $(\mathrm{P}<0.05)$ 
RELACIONES ANTERIORMENTE ESTABLECIDAS EN LOS PACIENTES QUE RECIBIERON UNA LAPAROTOMÍA:

Las siguientes tablas muestran las relaciones descritas con anterioridad pero sólo en los pacientes que recibieron una laparotomía:

\begin{tabular}{|c|c|c|c|}
\hline \multicolumn{4}{|c|}{$\begin{array}{l}\text { Tabla de quir_horas_por MORTALIDAD } \\
\text { quir_horas_ } \\
\text { MORTALIDAD (MORTALIDAD) }\end{array}$} \\
\hline $\begin{array}{l}\text { Frecuencia } \\
\text { Pct fila }\end{array}$ & o| & 1 & Total \\
\hline de00a01 & $\begin{array}{r}0 \\
0.00\end{array}$ & $\begin{array}{r}4 \\
100.00\end{array}$ & 4 \\
\hline de01a02 & $\begin{array}{r}17 \\
73.91\end{array}$ & $\begin{array}{r}6 \\
26.09\end{array}$ & 23 \\
\hline de02a03 & $\begin{array}{r}36 \\
83.72\end{array}$ & $\begin{array}{r}7 \\
16.28\end{array}$ & 43 \\
\hline de03a04 & $\begin{array}{r}24 \\
80.00\end{array}$ & $\begin{array}{r}6 \\
20.00\end{array}$ & 30 \\
\hline de04a99 & $\begin{array}{r}21 \\
95.45\end{array}$ & $\begin{array}{r}1 \\
4.55\end{array}$ & 22 \\
\hline Total & 98 & 24 & 122 \\
\hline
\end{tabular}

TABLA XXXIV: TIEMPO TOTAL DE QUIRÓFANO / MORTALIDAD EN LAPAROTOMIZADOS

\begin{tabular}{|c|c|c|c|}
\hline \multicolumn{4}{|c|}{$\begin{array}{c}\text { Tabla de quir_horas_por compli } \\
\text { quir_horas_ complicacion }\end{array}$} \\
\hline $\begin{array}{l}\text { Frecuencia } \\
\text { Pct fila }\end{array}$ & NO & SI & Total \\
\hline de00a01 & $\begin{array}{r}0 \\
0.00\end{array}$ & $\begin{array}{r}4 \\
100.00\end{array}$ & 4 \\
\hline de01a02 & $\begin{array}{r}15 \\
65.22\end{array}$ & $\begin{array}{r}8 \\
34.78\end{array}$ & 23 \\
\hline de02a03 & $\begin{array}{r}31 \\
72.09\end{array}$ & $\begin{array}{r}12 \\
27.91\end{array}$ & 43 \\
\hline de03a04 & $\begin{array}{r}18 \\
60.00\end{array}$ & $\begin{array}{r}12 \\
40.00\end{array}$ & 30 \\
\hline de04a99 & $\begin{array}{r}12 \\
54.55\end{array}$ & $\begin{array}{r}10 \\
45.45\end{array}$ & 22 \\
\hline Total & 76 & 46 & 122 \\
\hline
\end{tabular}

TABLA XXXV: TIEMPO TOTAL DE QUIRÓFANO / COMPLICACIONES EN LAPAROTOMIZADOS 


\begin{tabular}{|c|c|c|c|}
\hline \multicolumn{4}{|c|}{$\begin{array}{l}\text { abla de quir_horas_ por REINTERVENCION } \\
\text { quir_horas_ }\end{array}$} \\
\hline Frecuencia & & & \\
\hline Pct fila & NO & |SI & Total \\
\hline de00a01 & $\begin{array}{r}3 \\
75.00\end{array}$ & $\begin{array}{r}1 \\
25.00\end{array}$ & 4 \\
\hline de01a02 & $\begin{array}{r}17 \\
73.91\end{array}$ & $\begin{array}{r}6 \\
26.09\end{array}$ & 23 \\
\hline de02a03 & $\begin{array}{r}25 \\
58.14\end{array}$ & $\begin{array}{r}18 \\
41.86\end{array}$ & 43 \\
\hline de03a04 & $\begin{array}{r}18 \\
60.00\end{array}$ & $\begin{array}{r}12 \\
40.00\end{array}$ & 30 \\
\hline de04a99 & $\begin{array}{r}14 \\
63.64\end{array}$ & $\begin{array}{r}8 \\
36.36\end{array}$ & 22 \\
\hline Total & 77 & 45 & 122 \\
\hline
\end{tabular}

TABLA XXXVI: TIEMPO TOTAL DE QUIRÓFANO/REINTERVENCIÓN EN LAPAROTOMIZADOS

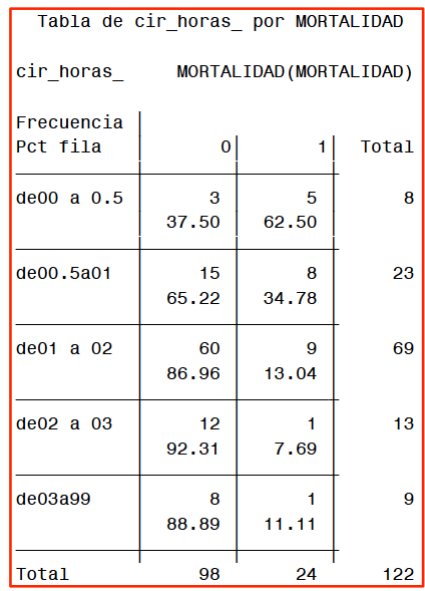

TABLA XXXVII: TIEMPO QUIRÚRGICO / MORTALIDAD EN LAPAROTOMIZADOS 


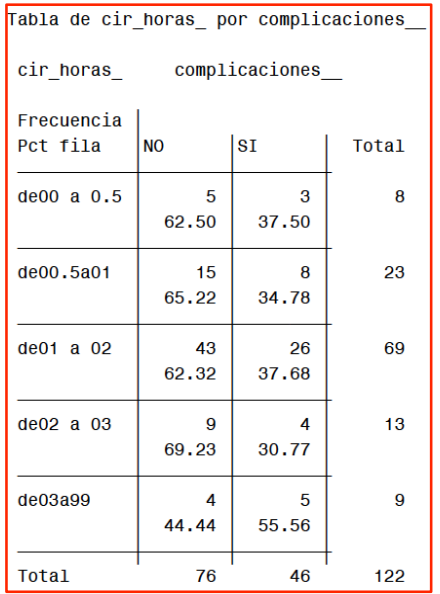

TABLA XXXVIII: TIEMPO QUIRÚRGICO/ COMPLICACIONES EN LAPAROTOMIZADOS

\begin{tabular}{|c|c|c|c|}
\hline \multirow{2}{*}{$\begin{array}{l}\text { Tabla de ci } \\
\text { cir_noras_ } \\
\text { Frecuencia } \\
\text { Pct fila }\end{array}$} & \multicolumn{3}{|c|}{ REINTERVENCION (RE INTERVENCION) } \\
\hline & NO & SI & Total \\
\hline de 00 a 0.5 & $\begin{array}{r}7 \\
87.50\end{array}$ & $\begin{array}{r}1 \\
12.50\end{array}$ & 8 \\
\hline de $00.5 \mathrm{a} 01$ & $\begin{array}{r}14 \\
60.87\end{array}$ & $\begin{array}{r}9 \\
39.13\end{array}$ & 23 \\
\hline de01 a 02 & $\begin{array}{r}43 \\
62.32\end{array}$ & $\begin{array}{r}26 \\
37.68\end{array}$ & 69 \\
\hline de02 a 03 & $\begin{array}{r}8 \\
61.54\end{array}$ & $\begin{array}{r}5 \\
38.46\end{array}$ & 13 \\
\hline de03a99 & $\begin{array}{r}5 \\
55.56\end{array}$ & $\begin{array}{r}4 \\
44.44\end{array}$ & 9 \\
\hline Total & 77 & 45 & 122 \\
\hline
\end{tabular}

TABLA XXXIX: TIEMPO QUIRÚRGICO / REINTERVENCIÓN EN LAPAROTOMIZADOS 


\begin{tabular}{|c|c|c|c|c|c|c|c|}
\hline $\begin{array}{l}\text { quir_horas } \\
\text { Frecuencia } \\
\text { Pct fila }\end{array}$ & $\begin{array}{r}\text { T } \\
-\quad \text { esta } \\
\text { de000a00 } \\
7\end{array}$ & $\begin{array}{l}\text { Tabla de } \\
\text { ancia_ }\end{array}$ & quir_horas & s_por es & $\left|\begin{array}{l}\text { de061a12 } \\
0\end{array}\right|$ & $\left|\begin{array}{l}\text { de121 a99 } \\
9\end{array}\right|$ & Total \\
\hline de00a01 & $\begin{array}{r}2 \\
50.00\end{array}$ & $\begin{array}{r}1 \\
25.00\end{array}$ & $\begin{array}{r}0 \\
0.00\end{array}$ & $\begin{array}{r}1 \\
25.00\end{array}$ & $\begin{array}{r}0 \\
0.00\end{array}$ & $\begin{array}{r}0 \\
0.00\end{array}$ & 4 \\
\hline de01a02 & $\begin{array}{r}2 \\
8.70\end{array}$ & $\begin{array}{r}4 \\
17.39\end{array}$ & $\begin{array}{r}6 \\
26.09\end{array}$ & $\begin{array}{r}5 \\
21.74\end{array}$ & $\begin{array}{r}4 \\
17.39\end{array}$ & $\begin{array}{r}2 \\
8.70\end{array}$ & 23 \\
\hline de02a03 & $\begin{array}{r}1 \\
2.33\end{array}$ & $\begin{array}{r}4 \\
9.30\end{array}$ & $\begin{array}{r}14 \\
32.56\end{array}$ & $\begin{array}{r}8 \\
18.60\end{array}$ & $\begin{array}{r}10 \\
23.26\end{array}$ & $\begin{array}{r}6 \\
13.95\end{array}$ & 43 \\
\hline de03a04 & $\begin{array}{r}0 \\
0.00\end{array}$ & $\begin{array}{r}5 \\
16.67\end{array}$ & $\begin{array}{r}9 \\
30.00\end{array}$ & $\begin{array}{r}5 \\
16.67\end{array}$ & $\begin{array}{r}9 \\
30.00\end{array}$ & $\begin{array}{r}2 \\
6.67\end{array}$ & 30 \\
\hline de04a99 & $\begin{array}{r}1 \\
4.55\end{array}$ & $\begin{array}{r}4 \\
18.18\end{array}$ & $\begin{array}{r}4 \\
18.18\end{array}$ & $\begin{array}{r}3 \\
13.64\end{array}$ & $\begin{array}{r}5 \\
22.73\end{array}$ & $\begin{array}{r}5 \\
22.73\end{array}$ & 22 \\
\hline Total & 6 & 18 & 33 & 22 & 28 & 15 & 122 \\
\hline
\end{tabular}

TABLA XL: TIEMPO TOTAL DE QUIRÓFANO / TIEMPO DE INGRESO EN LAPAROTOMIZADOS

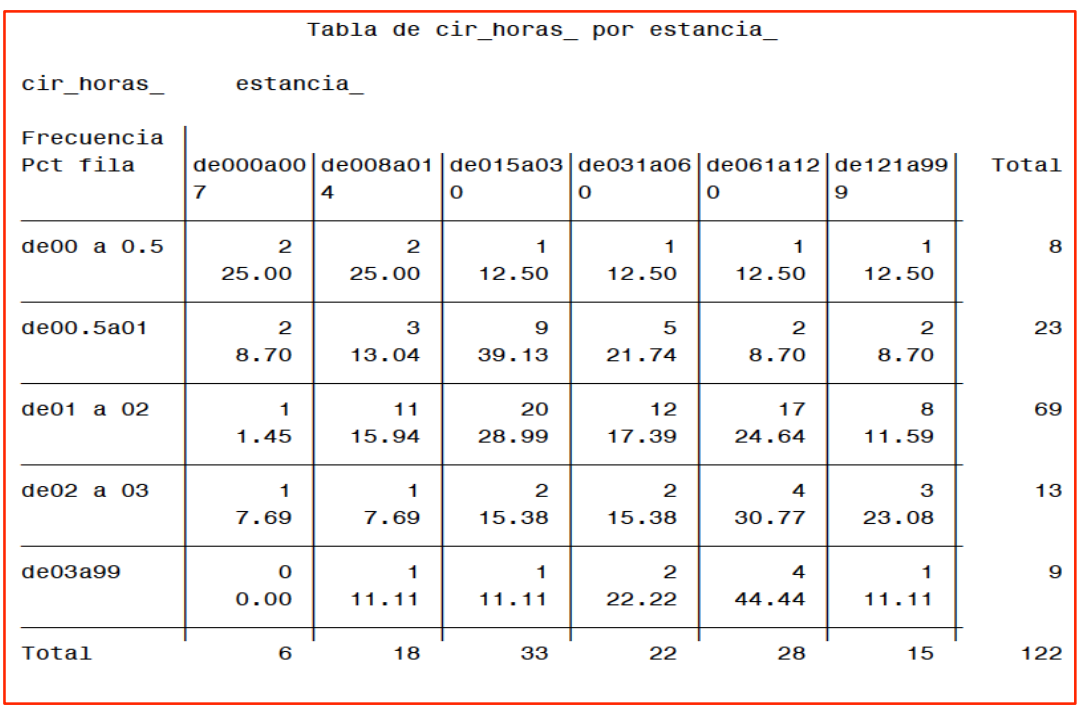

TABLA XLI: TIEMPO QUIRÚRGICO/ TIEMPO DE INGRESO EN LAPAROTOMIZADOS 


\begin{tabular}{|c|c|c|c|c|c|c|c|}
\hline \multicolumn{8}{|c|}{$\begin{array}{l}\text { Tabla de quir_horas_por TIEMPO_UCIN_ } \\
\text { TIEMPO_UCIN_ }\end{array}$} \\
\hline $\begin{array}{l}\text { Frecuencia } \\
\text { Pct fila }\end{array}$ & de00a01 & de02a03 & de04a07 & de07a14 & de15a30 & |de31a99 & Total \\
\hline de00a01 & $\begin{array}{r}1 \\
25.00\end{array}$ & $\begin{array}{r}0 \\
0.00\end{array}$ & $\begin{array}{r}0 \\
0.00\end{array}$ & $\begin{array}{r}2 \\
50.00\end{array}$ & $\begin{array}{r}0 \\
0.00\end{array}$ & $\begin{array}{r}1 \\
25.00\end{array}$ & 4 \\
\hline de01a02 & $\begin{array}{r}4 \\
17.39\end{array}$ & $\begin{array}{r}2 \\
8.70\end{array}$ & $\begin{array}{r}3 \\
13.04\end{array}$ & $\begin{array}{r}5 \\
21.74\end{array}$ & $\begin{array}{r}6 \\
26.09\end{array}$ & $\begin{array}{r}3 \\
13.04\end{array}$ & 23 \\
\hline de02a03 & $\begin{array}{r}7 \\
16.28\end{array}$ & $\begin{array}{r}6 \\
13.95\end{array}$ & $\begin{array}{r}6 \\
13.95\end{array}$ & $\begin{array}{r}7 \\
16.28\end{array}$ & $\begin{array}{r}6 \\
13.95\end{array}$ & $\begin{array}{r}11 \\
25.58\end{array}$ & 43 \\
\hline de03a04 & $\begin{array}{r}1 \\
3.33\end{array}$ & $\begin{array}{r}3 \\
10.00\end{array}$ & $\begin{array}{r}7 \\
23.33\end{array}$ & $\begin{array}{r}6 \\
20.00\end{array}$ & $\begin{array}{r}9 \\
30.00\end{array}$ & $\begin{array}{r}4 \\
13.33\end{array}$ & 30 \\
\hline de04a99 & $\begin{array}{r}4 \\
18.18\end{array}$ & $\begin{array}{r}3 \\
13.64\end{array}$ & $\begin{array}{r}3 \\
13.64\end{array}$ & $\begin{array}{r}3 \\
13.64\end{array}$ & $\begin{array}{r}3 \\
13.64\end{array}$ & $\begin{array}{r}6 \\
27.27\end{array}$ & 22 \\
\hline Total & 17 & 14 & 19 & 23 & 24 & 25 & 122 \\
\hline
\end{tabular}

TABLA XLII: TIEMPO TOTAL DE QUIRÓFANO / TIEMPO DE INGRESO EN UCIN EN LAPAROTOMIZADOS

\begin{tabular}{|c|c|c|c|c|c|c|c|}
\hline $\begin{array}{l}\text { cir_horas_ } \\
\text { Frecuencia } \\
\text { Pct fila }\end{array}$ & de00a01 & $\begin{array}{l}\text { abla de c } \\
\text { O_ucIN_ }\end{array}$ & ir_horas & por TIEN & IPO_UCIN_ & de31a99 & Total \\
\hline de00 a 0.5 & $\begin{array}{r}2 \\
25.00\end{array}$ & $\begin{array}{r}0 \\
0.00\end{array}$ & $\begin{array}{r}2 \\
25.00\end{array}$ & $\begin{array}{r}2 \\
25.00\end{array}$ & $\begin{array}{r}1 \\
12.50\end{array}$ & $\begin{array}{r}1 \\
12.50\end{array}$ & 8 \\
\hline de00.5a01 & $\begin{array}{r}5 \\
21.74\end{array}$ & $\begin{array}{r}3 \\
13.04\end{array}$ & $\begin{array}{r}3 \\
13.04\end{array}$ & $\begin{array}{r}4 \\
17.39\end{array}$ & $\begin{array}{r}4 \\
17.39\end{array}$ & $\begin{array}{r}4 \\
17.39\end{array}$ & 23 \\
\hline de01 a 02 & $\begin{array}{r}7 \\
10.14\end{array}$ & $\begin{array}{r}9 \\
13.04\end{array}$ & $\begin{array}{r}12 \\
17.39\end{array}$ & $\begin{array}{r}13 \\
18.84\end{array}$ & $\begin{array}{r}15 \\
21.74\end{array}$ & $\begin{array}{r}13 \\
18.84\end{array}$ & 69 \\
\hline de02 a 03 & $\begin{array}{r}1 \\
7.69\end{array}$ & $\begin{array}{r}2 \\
15.38\end{array}$ & $\begin{array}{r}0 \\
0.00\end{array}$ & $\begin{array}{r}4 \\
30.77\end{array}$ & $\begin{array}{r}2 \\
15.38\end{array}$ & $\begin{array}{r}4 \\
30.77\end{array}$ & 13 \\
\hline de03a99 & $\begin{array}{r}2 \\
22.22\end{array}$ & $\begin{array}{r}0 \\
0.00\end{array}$ & $\begin{array}{r}2 \\
22.22\end{array}$ & $\begin{array}{r}0 \\
0.00\end{array}$ & $\begin{array}{r}2 \\
22.22\end{array}$ & $\begin{array}{r}3 \\
33.33\end{array}$ & 9 \\
\hline Total & 17 & 14 & 19 & 23 & 24 & 25 & 122 \\
\hline
\end{tabular}

TABLA XLIII: TIEMPO QUIRÚRGICO/TIEMPO DE INGRESO EN UCIN EN LAPAROTOMIZADOS

Existe una relación estadísticamente significativa entre tiempo quirúrgico y mortalidad $\mathrm{P}=0.0029(\mathrm{P}<0.05)$ y tiempo de ingreso $\mathrm{P}=$ $0.0052(P<0.05)$ y entre tiempo total de quirófano y mortalidad $P=0.0014$ $(\mathrm{P}<0.05)$ 
RELACIONES ANTERIORMENTE ESTABLECIDAS EN LOS PACIENTES QUE RECIBIERON UNA TORACOTOMÍA:

Las siguientes tablas muestran las relaciones descritas con anterioridad pero sólo en los pacientes que recibieron una toracotomía:

\begin{tabular}{|c|c|c|c|}
\hline \multirow{2}{*}{$\begin{array}{l}\text { Tabla de qu } \\
\text { quir_horas } \\
\text {-recuencia } \\
\text { Pct fila }\end{array}$} & \multicolumn{3}{|c|}{ MORTAL IDAD (MORTAL IDAD) } \\
\hline & 0 & 1 & Total \\
\hline de00a01 & $\begin{array}{r}8 \\
88.89\end{array}$ & $\begin{array}{r}1 \\
11.11\end{array}$ & 9 \\
\hline de01a02 & $\begin{array}{r}23 \\
88.46\end{array}$ & $\begin{array}{r}3 \\
11.54\end{array}$ & 26 \\
\hline 1е02а0з & $\begin{array}{r}3 \\
75.00\end{array}$ & $\begin{array}{r}1 \\
25.00\end{array}$ & 4 \\
\hline деоза04 & $\begin{array}{r}4 \\
66.67\end{array}$ & $\begin{array}{r}2 \\
33.33\end{array}$ & 6 \\
\hline de04a99 & $\begin{array}{r}6 \\
85.71\end{array}$ & $\begin{array}{r}1 \\
14.29\end{array}$ & 7 \\
\hline Total & 44 & 8 & 52 \\
\hline
\end{tabular}

TABLA XLIV: TIEMPO TOTAL DE QUIRÓFANO / MORTALIDAD EN TORACOTOMIZADOS

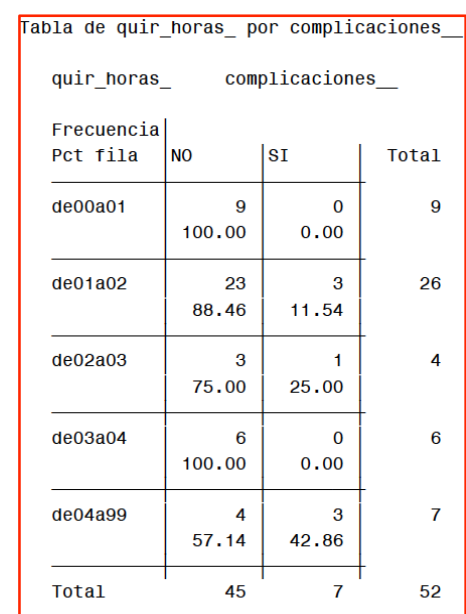

TABLA XLV: TIEMPO TOTAL DE QUIRÓFANO / COMPLICACIONES EN TORACOTOMIZADOS 


\begin{tabular}{|c|c|c|c|}
\hline $\begin{array}{l}\text { Tabla de quir } \\
\text { quir_horas }\end{array}$ & \multicolumn{3}{|c|}{ REINTERVENCION (REINTERVENCION) } \\
\hline $\begin{array}{l}\text { Frecuencia } \\
\text { Pct fila }\end{array}$ & No & SI & Total \\
\hline de00a01 & $\begin{array}{r}9 \\
100.00\end{array}$ & $\begin{array}{r}0 \\
0.00\end{array}$ & 9 \\
\hline de01a02 & $\begin{array}{r}25 \\
96.15\end{array}$ & $\begin{array}{r}1 \\
3.85\end{array}$ & 26 \\
\hline de02a03 & $\begin{array}{r}3 \\
75.00\end{array}$ & $\begin{array}{r}1 \\
25.00\end{array}$ & 4 \\
\hline de03a04 & $\begin{array}{r}5 \\
83.33\end{array}$ & $\begin{array}{r}1 \\
16.67\end{array}$ & 6 \\
\hline de04a99 & $\begin{array}{r}4 \\
57.14\end{array}$ & $\begin{array}{r}3 \\
42.86\end{array}$ & 7 \\
\hline Total & 46 & 6 & 52 \\
\hline
\end{tabular}

TABLA XLVI: TIEMPO TOTAL DE QUIRÓFANO / REINTERVENCIÓN EN TORACOTOMIZADOS

\begin{tabular}{|c|c|c|c|}
\hline \multirow{2}{*}{$\begin{array}{l}\text { cir_horas_ } \\
\text { Frecuencia } \\
\text { Pct fila }\end{array}$} & \multicolumn{3}{|c|}{ MORTALIDAD (MORTALIDAD) } \\
\hline & 0 & $1 \mid$ & Total \\
\hline de 00 a 0.5 & $\begin{array}{r}20 \\
90.91\end{array}$ & $\begin{array}{r}2 \\
9.09\end{array}$ & 22 \\
\hline de00.5a01 & $\begin{array}{r}13 \\
86.67\end{array}$ & $\begin{array}{r}2 \\
13.33\end{array}$ & 15 \\
\hline de01 a 02 & $\begin{array}{r}4 \\
57.14\end{array}$ & $\begin{array}{r}3 \\
42.86\end{array}$ & 7 \\
\hline de02 a 03 & $\begin{array}{r}5 \\
83.33\end{array}$ & $\begin{array}{r}1 \\
16.67\end{array}$ & 6 \\
\hline деоза99 & $\begin{array}{r}2 \\
100.00\end{array}$ & $\begin{array}{r}0 \\
0.00\end{array}$ & 2 \\
\hline Total & 44 & 8 & 52 \\
\hline
\end{tabular}

TABLA XLVII: TIEMPO QUIRÚRGICO / MORTALIDAD EN TORACOTOMIZADOS 


\begin{tabular}{|c|c|c|c|}
\hline \multicolumn{4}{|c|}{$\begin{array}{l}\text { Tabla de cir_horas_por complicaciones } \\
\text { cir_horas_ complicaciones }\end{array}$} \\
\hline $\begin{array}{l}\text { Frecuencia } \\
\text { Pct fila }\end{array}$ & NO & |SI & Total \\
\hline de00 a 0.5 & $\begin{array}{r}21 \\
95.45\end{array}$ & $\begin{array}{r}1 \\
4.55\end{array}$ & 22 \\
\hline de00.5a01 & $\begin{array}{r}12 \\
80.00\end{array}$ & $\begin{array}{r}3 \\
20.00\end{array}$ & 15 \\
\hline de 01 a 02 & $\begin{array}{r}6 \\
85.71\end{array}$ & $\begin{array}{r}1 \\
14.29\end{array}$ & 7 \\
\hline de02 a 03 & $\begin{array}{r}5 \\
83.33\end{array}$ & $\begin{array}{r}1 \\
16.67\end{array}$ & 6 \\
\hline de03a99 & $\begin{array}{r}1 \\
50.00\end{array}$ & $\begin{array}{r}1 \\
50.00\end{array}$ & 2 \\
\hline Total & 45 & 7 & 52 \\
\hline
\end{tabular}

TABLA XLVIII: TIEMPO QUIRÚRGICO / COMPLICACIONES EN TORACOTOMIZADOS

\begin{tabular}{|c|c|c|c|}
\hline \multirow{2}{*}{$\begin{array}{l}\text { Tabla de ci } \\
\text { cir_horas_ } \\
\text { Frecuencia } \\
\text { Pct fila }\end{array}$} & \multicolumn{3}{|c|}{ REINTERVENCION (REINTERVENCION) } \\
\hline & No & |SI & Total \\
\hline de00 a 0.5 & $\begin{array}{r}22 \\
100.00\end{array}$ & $\begin{array}{r}0 \\
0.00\end{array}$ & 22 \\
\hline de $00.5 a 01$ & $\begin{array}{r}13 \\
86.67\end{array}$ & $\begin{array}{r}2 \\
13.33\end{array}$ & 15 \\
\hline de01 a 02 & $\begin{array}{r}5 \\
71.43\end{array}$ & $\begin{array}{r}2 \\
28.57\end{array}$ & 7 \\
\hline de02 a 03 & $\begin{array}{r}5 \\
83.33\end{array}$ & $\begin{array}{r}1 \\
16.67\end{array}$ & 6 \\
\hline deоза99 & $\begin{array}{r}1 \\
50.00\end{array}$ & $\begin{array}{r}1 \\
50.00\end{array}$ & 2 \\
\hline Total & 46 & 6 & 52 \\
\hline
\end{tabular}

TABLA IL: TIEMPO QUIRÚRGICO /REINTERVENCIÓN EN TORACOTOMIZADOS 


\begin{tabular}{|c|c|c|c|c|c|c|c|}
\hline $\begin{array}{l}\text { quir_horas } \\
\text { Frecuencia } \\
\text { Pct fila }\end{array}$ & $\begin{array}{l}\text { de000a00 } \\
7\end{array}$ & $\begin{array}{l}\text { Tabla de } \\
\text { ancia_ } \\
\mid \begin{array}{l}\text { de008a01 } \\
4\end{array}\end{array}$ & quir_horas & $\left|\begin{array}{l}\text { de031a06 } \\
0\end{array}\right|$ & $\left|\begin{array}{l}\text { de061a12 } \\
0\end{array}\right|$ & $\left|\begin{array}{l}\text { de121a99 } \\
9\end{array}\right|$ & Total \\
\hline de00a01 & $\begin{array}{r}0 \\
0.00\end{array}$ & $\begin{array}{r}0 \\
0.00\end{array}$ & $\begin{array}{r}0 \\
0.00\end{array}$ & $\begin{array}{r}0 \\
0.00\end{array}$ & $\begin{array}{r}5 \\
55.56\end{array}$ & $\begin{array}{r}4 \\
44.44\end{array}$ & 9 \\
\hline de01a02 & $\begin{array}{r}1 \\
3.85\end{array}$ & $\begin{array}{r}1 \\
3.85\end{array}$ & $\begin{array}{r}0 \\
0.00\end{array}$ & $\begin{array}{r}3 \\
11.54\end{array}$ & $\begin{array}{r}12 \\
46.15\end{array}$ & $\begin{array}{r}9 \\
34.62\end{array}$ & 26 \\
\hline de02a03 & $\begin{array}{r}0 \\
0.00\end{array}$ & $\begin{array}{r}0 \\
0.00\end{array}$ & $\begin{array}{r}0 \\
0.00\end{array}$ & $\begin{array}{r}1 \\
25.00\end{array}$ & $\begin{array}{r}2 \\
50.00\end{array}$ & $\begin{array}{r}1 \\
25.00\end{array}$ & 4 \\
\hline de03a04 & $\begin{array}{r}0 \\
0.00\end{array}$ & $\begin{array}{r}2 \\
33.33\end{array}$ & $\begin{array}{r}1 \\
16.67\end{array}$ & $\begin{array}{r}0 \\
0.00\end{array}$ & $\begin{array}{r}1 \\
16.67\end{array}$ & $\begin{array}{r}2 \\
33.33\end{array}$ & 6 \\
\hline de04a99 & $\begin{array}{r}0 \\
0.00\end{array}$ & $\begin{array}{r}0 \\
0.00\end{array}$ & $\begin{array}{r}4 \\
57.14\end{array}$ & $\begin{array}{r}1 \\
14.29\end{array}$ & $\begin{array}{r}1 \\
14.29\end{array}$ & $\begin{array}{r}1 \\
14.29\end{array}$ & 7 \\
\hline Total & 1 & 3 & 5 & 5 & 21 & 17 & 52 \\
\hline
\end{tabular}

TABLA L: TIEMPO TOTAL DE QUIRÓFANO / TIEMPO DE INGRESO EN TORACOTOMIZADOS

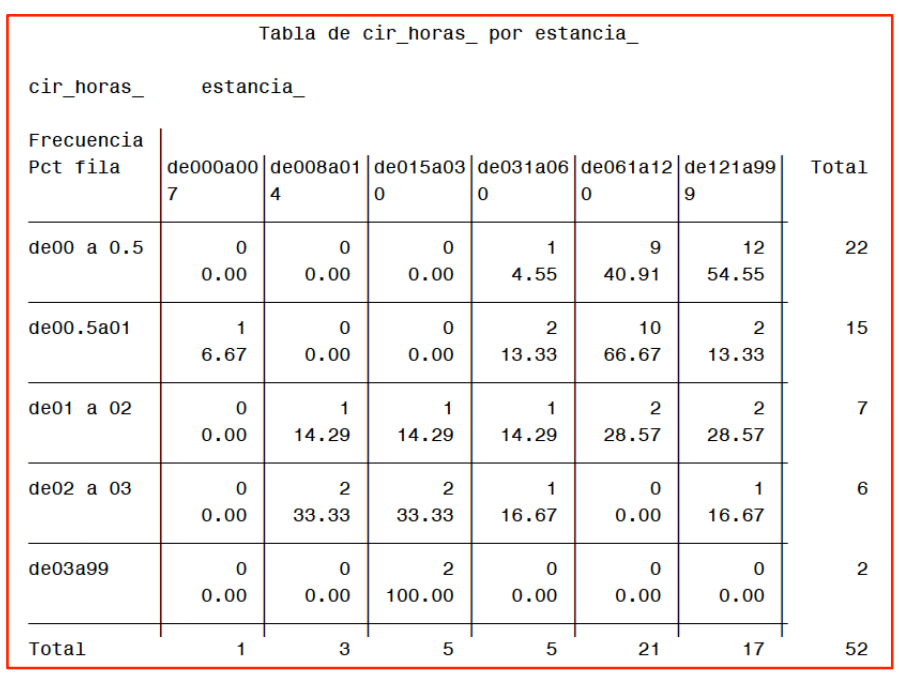

TABLA LI: TIEMPO QUIRÚRGICO / TIEMPO DE INGRESO EN TORACOTOMIZADOS 


\begin{tabular}{|c|c|c|c|c|c|c|c|}
\hline \multicolumn{8}{|c|}{$\begin{array}{l}\text { Tabla de quir_horas_por TIEMPO_UCIN_ } \\
\text { TIEMPO_UCIN }\end{array}$} \\
\hline $\begin{array}{l}\text { Frecuencia } \\
\text { Pct fila }\end{array}$ & de00a01 & de02a03 & de $04 \mathrm{a} 07$ & de $07 a_{14}$ & de15a30 & de31a99 & Total \\
\hline de00a01 & $\begin{array}{r}0 \\
0.00\end{array}$ & $\begin{array}{r}0 \\
0.00\end{array}$ & $\begin{array}{r}0 \\
0.00\end{array}$ & $\begin{array}{r}0 \\
0.00\end{array}$ & $\begin{array}{r}1 \\
11.11\end{array}$ & $\begin{array}{r}8 \\
88.89\end{array}$ & 9 \\
\hline de01a02 & $\begin{array}{r}1 \\
3.85\end{array}$ & $\begin{array}{r}1 \\
3.85\end{array}$ & $\begin{array}{r}1 \\
3.85\end{array}$ & $\begin{array}{r}1 \\
3.85\end{array}$ & $\begin{array}{r}4 \\
15.38\end{array}$ & $\begin{array}{r}18 \\
69.23\end{array}$ & 26 \\
\hline de02a03 & $\begin{array}{r}0 \\
0.00\end{array}$ & $\begin{array}{r}0 \\
0.00\end{array}$ & $\begin{array}{r}0 \\
0.00\end{array}$ & $\begin{array}{r}0 \\
0.00\end{array}$ & $\begin{array}{r}0 \\
0.00\end{array}$ & $\begin{array}{r}4 \\
100.00\end{array}$ & 4 \\
\hline de03a04 & $\begin{array}{r}0 \\
0.00\end{array}$ & $\begin{array}{r}1 \\
16.67\end{array}$ & $\begin{array}{r}1 \\
16.67\end{array}$ & $\begin{array}{r}2 \\
33.33\end{array}$ & $\begin{array}{r}0 \\
0.00\end{array}$ & $\begin{array}{r}2 \\
33.33\end{array}$ & 6 \\
\hline de04a99 & $\begin{array}{r}0 \\
0.00\end{array}$ & $\begin{array}{r}0 \\
0.00\end{array}$ & $\begin{array}{r}1 \\
14.29\end{array}$ & $\begin{array}{r}4 \\
57.14\end{array}$ & $\begin{array}{r}1 \\
14.29\end{array}$ & $\begin{array}{r}1 \\
14.29\end{array}$ & 7 \\
\hline Total & 1 & 2 & 3 & 7 & 6 & 33 & 52 \\
\hline
\end{tabular}

TABLA LII: TIEMPO TOTAL DE QUIRÓFANO/ TIEMPO DE INGRESO EN UCIN EN TORACOTOMIZADOS

\begin{tabular}{|l|r|r|r|r|r|r|r|}
\hline \multicolumn{7}{|c|}{ Tabla de cir_horas_por TIEMPO_UCIN_ } \\
cir_horas_ \\
$\begin{array}{l}\text { Frecuencia } \\
\text { Pct fila }\end{array}$ & de00a01 & de02a03 & de04a07 & de07a14 & de15a30 & de31a99 & Total \\
\hline de00 a 0.5 & 0 & 0 & 1 & 0 & 3 & 18 & 22 \\
& 0.00 & 0.00 & 4.55 & 0.00 & 13.64 & 81.82 & \\
\hline de00.5a01 & 1 & 1 & 0 & 0 & 2 & 11 & 15 \\
\hline de01 a 02 & 6.67 & 6.67 & 0.00 & 0.00 & 13.33 & 73.33 & \\
\hline de02 a 03 & 0.00 & 14.29 & 0.00 & 42.86 & 0.00 & 42.86 & 7 \\
\hline de03a99 & 0.00 & 0.00 & 33.33 & 33.33 & 16.67 & 16.67 & 6 \\
\hline Total & 0.00 & 0.00 & 0.00 & 100.00 & 0.00 & 0.00 & 2 \\
\hline
\end{tabular}

TABLA LIII: TIEMPO QUIRÚRGICO/ TIEMPO DE INGRESO EN UCIN EN TORACOTOMIZADOS

Existe una relación estadísticamente significativa entre tiempo quirúrgico y reintervención $\mathrm{P}=0.041$, tiempo en UCIN $\mathrm{p}=0.0068$ y tiempo de ingreso $\mathrm{P}=0.001$ y del tiempo total de quirófano con las complicaciones, $\mathrm{P}=\mathbf{0 . 0 0 9 4}$ reintervención $\mathrm{P}=\mathbf{0 . 0 0 2 1}$, tiempo de ingreso en UCIN $P=0.0068$ y tiempo de ingreso total. $P=0.0016$ 
RELACIONES ANTERIORMENTE ESTABLECIDAS EN LOS PACIENTES

DIAGNOSTICADOS DE ENTEROCOLITIS NECROTIZANTE:

Las siguientes tablas muestran las relaciones descritas con anterioridad pero sólo en los pacientes diagnosticados de Enterocolitis Necrotizante:

\begin{tabular}{|c|c|c|c|}
\hline \multicolumn{4}{|c|}{$\begin{array}{l}\text { Tabla de quir_horas_por MORTALIDAD } \\
\text { quir_horas__ } \\
{ }_{\text {MORTALIDAD (MORTALIDAD) }}\end{array}$} \\
\hline $\begin{array}{l}\text { Frecuencia } \\
\text { Pct rila }\end{array}$ & 0 & 1 & Total \\
\hline de00a01 & $\begin{array}{r}0 \\
0.00\end{array}$ & $\begin{array}{r}4 \\
100.00\end{array}$ & 4 \\
\hline de01a02 & $\begin{array}{r}6 \\
46.15\end{array}$ & $\begin{array}{r}7 \\
53.85\end{array}$ & 13 \\
\hline de02a03 & $\begin{array}{r}9 \\
60.00\end{array}$ & $\begin{array}{r}6 \\
40.00\end{array}$ & 15 \\
\hline de03a04 & $\begin{array}{r}4 \\
44.44\end{array}$ & $\begin{array}{r}5 \\
55.56\end{array}$ & 9 \\
\hline de04a99 & $\begin{array}{r}2 \\
100.00\end{array}$ & $\begin{array}{r}0 \\
0.00\end{array}$ & 2 \\
\hline Total & 21 & 22 & 43 \\
\hline
\end{tabular}

TABLA LIV: TIEMPO TOTAL DE QUIRÓFANO / MORTALIDAD EN ENTEROCOLITIS

\begin{tabular}{|c|c|c|c|}
\hline \multicolumn{4}{|c|}{ licaciones } \\
\hline quir_horas & con & plicacion & \\
\hline $\begin{array}{l}\text { Frecuencia } \\
\text { Pct fila }\end{array}$ & No & SI & Total \\
\hline de00a01 & $\begin{array}{r}0 \\
0.00\end{array}$ & $\begin{array}{r}4 \\
100.00\end{array}$ & 4 \\
\hline de01a02 & $\begin{array}{r}6 \\
46.15\end{array}$ & $\begin{array}{r}7 \\
53.85\end{array}$ & 13 \\
\hline de02a03 & $\begin{array}{r}6 \\
40.00\end{array}$ & $\begin{array}{r}9 \\
60.00\end{array}$ & 15 \\
\hline de03a04 & $\begin{array}{r}5 \\
55.56\end{array}$ & $\begin{array}{r}4 \\
44.44\end{array}$ & 9 \\
\hline de04a99 & $\begin{array}{r}0 \\
0.00\end{array}$ & $\begin{array}{r}2 \\
100.00\end{array}$ & 2 \\
\hline Total & 17 & 26 & 43 \\
\hline
\end{tabular}

TABLA LV: TIEMPO TOTAL DE QUIRÓFANO / COMPLICACIONES EN ENTEROCOLITIS 


\begin{tabular}{|c|c|c|c|}
\hline $\begin{array}{c}\text { rabla de quir } \\
\text { quir_horas }\end{array}$ & $\begin{array}{l}r_{\text {r_horas }} \\
\text { REINTERV }\end{array}$ & $\begin{array}{l}\text { por REINT } \\
\text { ENCION / RE }\end{array}$ & $\begin{array}{l}\text { VENCION } \\
\text { IERVENGION) }\end{array}$ \\
\hline $\begin{array}{l}\text { Frecuencia } \\
\text { Pct fila }\end{array}$ & No & |SI & Total \\
\hline de00a01 & $\begin{array}{r}3 \\
75.00\end{array}$ & $\begin{array}{r}1 \\
25.00\end{array}$ & 4 \\
\hline de01a02 & $\begin{array}{r}7 \\
53.85\end{array}$ & $\begin{array}{r}6 \\
46.15\end{array}$ & 13 \\
\hline de02a03 & $\begin{array}{r}4 \\
26.67\end{array}$ & $\begin{array}{r}11 \\
73.33\end{array}$ & 15 \\
\hline de03a04 & $\begin{array}{r}5 \\
55.56\end{array}$ & $\begin{array}{r}4 \\
44.44\end{array}$ & 9 \\
\hline de04a99 & $\begin{array}{r}0 \\
0.00\end{array}$ & $\begin{array}{r}2 \\
100.00\end{array}$ & 2 \\
\hline Total & 19 & 24 & 43 \\
\hline
\end{tabular}

TABLA LVI: TIEMPO TOTAL DE QUIRÓFANO / REINTERVENCIÓN EN ENTEROCOLITIS

\begin{tabular}{|c|c|c|c|}
\hline \multicolumn{4}{|c|}{ Tabla de cir_horas_por MORTALIDAD } \\
\hline cir_horas_ & \multicolumn{3}{|c|}{ MORTALIDAD (MORTALIDAD) } \\
\hline Pct fila & 이 & 1 & Total \\
\hline de00 a 0.5 & $\begin{array}{r}0 \\
0.00\end{array}$ & $\begin{array}{r}5 \\
100.00\end{array}$ & 5 \\
\hline de00.5a01 & $\begin{array}{r}2 \\
20.00\end{array}$ & $\begin{array}{r}8 \\
80.00\end{array}$ & 10 \\
\hline de01 a 02 & $\begin{array}{r}16 \\
69.57\end{array}$ & $\begin{array}{r}7 \\
30.43\end{array}$ & 23 \\
\hline de02 a 03 & $\begin{array}{r}2 \\
50.00\end{array}$ & $\begin{array}{r}2 \\
50.00\end{array}$ & 4 \\
\hline de03a99 & $\begin{array}{r}1 \\
100.00\end{array}$ & $\begin{array}{r}0 \\
0.00\end{array}$ & 1 \\
\hline Total & 21 & 22 & 43 \\
\hline
\end{tabular}

TABLA LVII: TIEMPO QUIRÚRGICO / MORTALIDAD EN ENTEROCOLITIS

\begin{tabular}{|c|c|c|c|}
\hline \multicolumn{4}{|c|}{ Tabla de cir_horas_por complicaciones } \\
\hline cir_horas_ & comp1 & icaciones & \\
\hline $\begin{array}{l}\text { Frecuencia } \\
\text { Pct fila }\end{array}$ & No & |SI & Total \\
\hline de00 a 0.5 & $\begin{array}{r}2 \\
40.00\end{array}$ & $\begin{array}{r}3 \\
60.00\end{array}$ & 5 \\
\hline de00.5a01 & $\begin{array}{r}3 \\
30.00\end{array}$ & $\begin{array}{r}7 \\
70.00\end{array}$ & 10 \\
\hline de01 a 02 & $\begin{array}{r}9 \\
39.13\end{array}$ & $\begin{array}{r}14 \\
60.87\end{array}$ & 23 \\
\hline de02 a 03 & $\begin{array}{r}3 \\
75.00\end{array}$ & $\begin{array}{r}1 \\
25.00\end{array}$ & 4 \\
\hline de03а99 & $\begin{array}{r}0 \\
0.00\end{array}$ & $\begin{array}{r}1 \\
100.00\end{array}$ & 1 \\
\hline Total & 17 & 26 & 43 \\
\hline
\end{tabular}

TABLA LVIII: TIEMPO QUIRÚRGICO /COMPLICACIONES EN ENTEROCOLITIS 


\begin{tabular}{|c|c|c|c|}
\hline \\
\hline \multicolumn{4}{|c|}{$\begin{array}{l}\text { Tabla de cir_horas_por REINTERVENCION } \\
\text { cir_horas_ } \\
\end{array}$} \\
\hline $\begin{array}{l}\text { Frecuencia } \\
\text { Pct fila }\end{array}$ & NO & SI & Total \\
\hline de 00 a 0.5 & $\begin{array}{r}5 \\
100.00\end{array}$ & $\begin{array}{r}0 \\
0.00\end{array}$ & 5 \\
\hline de00.5a01 & $\begin{array}{r}3 \\
30.00\end{array}$ & $\begin{array}{r}7 \\
70.00\end{array}$ & 10 \\
\hline de01 a 02 & $\begin{array}{r}9 \\
39.13\end{array}$ & $\begin{array}{r}14 \\
60.87\end{array}$ & 23 \\
\hline de02 a 03 & $\begin{array}{r}2 \\
50.00\end{array}$ & $\begin{array}{r}2 \\
50.00\end{array}$ & 4 \\
\hline de03a99 & $\begin{array}{r}0 \\
0.00\end{array}$ & $\begin{array}{r}1 \\
100.00\end{array}$ & 1 \\
\hline Total & 19 & 24 & 43 \\
\hline
\end{tabular}

TABLA LIX: TIEMPO QUIRÚRGICO/ REINTERVENCIÓN EN ENTEROCOLITIS

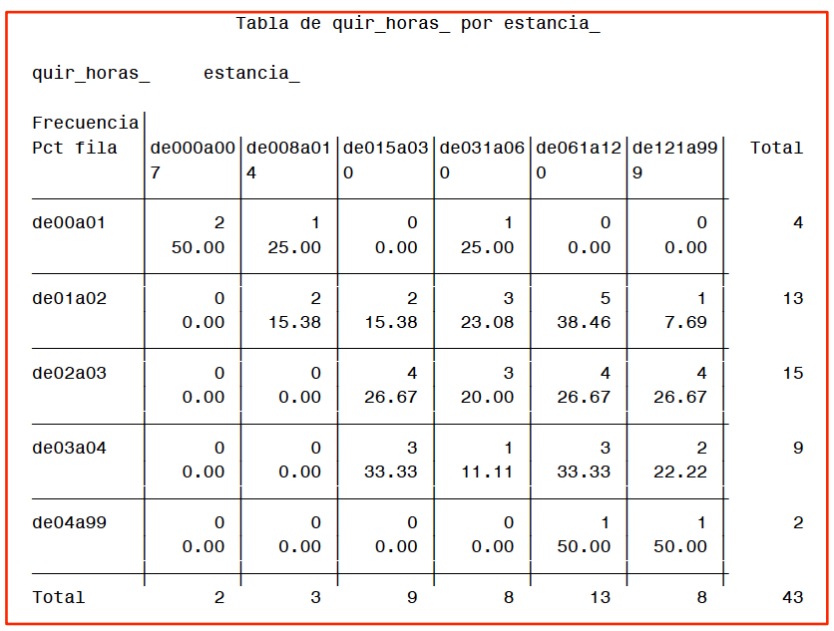

TABLA LX: TIEMPO TOTAL DE QUIRÓFANO / TIEMPO DE INGRESO EN ENTEROCOLITIS 


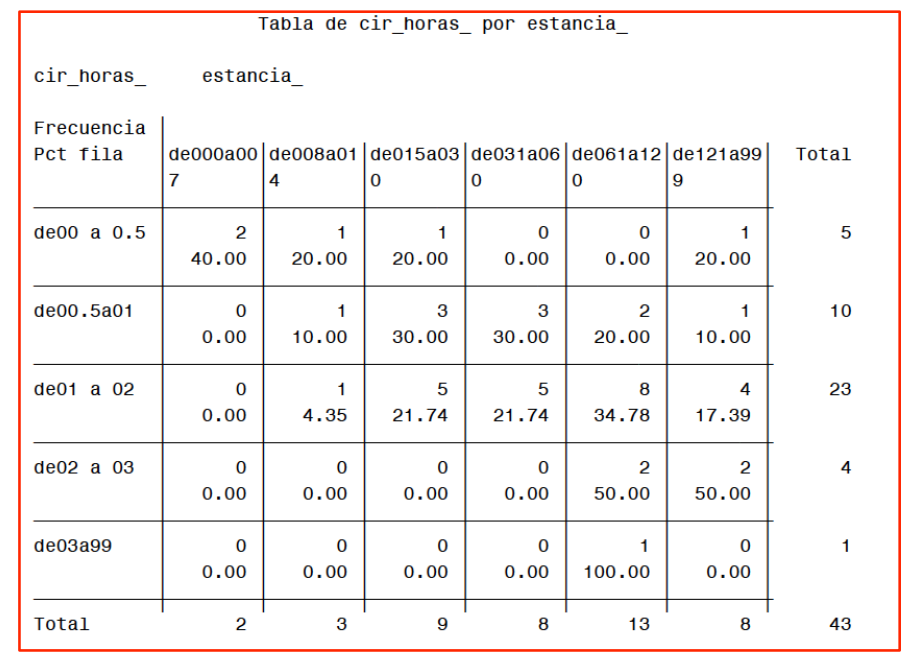

TABLA LXI: TIEMPO QUIRÚRGICO / TIEMPO DE INGRESO EN ENTEROCOLITIS

\begin{tabular}{|l|r|r|r|r|r|r|r|}
\hline \multicolumn{7}{|c|}{ Tabla de quir_horas_por TIEMPO_UCIN_ } \\
quir_horas_ \\
$\begin{array}{l}\text { Frecuencia } \\
\text { Pct fila }\end{array}$ & de00a01 de02a03 & de04a07 & de07a14 & de15a30 & de31a99 & Total \\
\hline de00a01 & 1 & 0 & 0 & 2 & 0 & 1 & 4 \\
& 25.00 & 0.00 & 0.00 & 50.00 & 0.00 & 25.00 & \\
\hline de01a02 & 1 & 0 & 0 & 4 & 3 & 5 & 13 \\
& 7.69 & 0.00 & 0.00 & 30.77 & 23.08 & 38.46 & \\
\hline de02a03 & 0 & 1 & 1 & 2 & 4 & 7 & 15 \\
\hline de03a04 & 0.00 & 6.67 & 6.67 & 13.33 & 26.67 & 46.67 & \\
\hline de04a99 & 0.00 & 11.11 & 0.00 & 22.22 & 33.33 & 33.33 & 9 \\
\hline Total & 1 & 0 & 0 & 0 & 1 & 0 & 2 \\
& 30.00 & 0.00 & 0.00 & 0.00 & 50.00 & 0.00 & \\
\hline
\end{tabular}

TABLA LXII: TIEMPO TOTAL DE QUIRÓFANO / TIEMPO DE INGRESO EN UCIN EN ENTEROCOLITIS 


\begin{tabular}{|c|c|c|c|c|c|c|c|}
\hline \multicolumn{8}{|c|}{ Tabla de cir_horas_por TIEMPO_UCIN_ } \\
\hline $\begin{array}{l}\text { Frecuencia } \\
\text { Pct fila }\end{array}$ & de00a01 & de02a03 & de04a07 & de07a14 & de15a30 & |de31a99 & Total \\
\hline de00 a 0.5 & $\begin{array}{r}1 \\
20.00\end{array}$ & $\begin{array}{r}0 \\
0.00\end{array}$ & $\begin{array}{r}0 \\
0.00\end{array}$ & $\begin{array}{r}2 \\
40.00\end{array}$ & $\begin{array}{r}1 \\
20.00\end{array}$ & $\begin{array}{r}1 \\
20.00\end{array}$ & 5 \\
\hline de00.5a01 & $\begin{array}{r}0 \\
0.00\end{array}$ & $\begin{array}{r}1 \\
10.00\end{array}$ & $\begin{array}{r}1 \\
10.00\end{array}$ & $\begin{array}{r}2 \\
20.00\end{array}$ & $\begin{array}{r}2 \\
20.00\end{array}$ & $\begin{array}{r}4 \\
40.00\end{array}$ & 10 \\
\hline de01 a 02 & $\begin{array}{r}1 \\
4.35\end{array}$ & $\begin{array}{r}1 \\
4.35\end{array}$ & $\begin{array}{r}0 \\
0.00\end{array}$ & $\begin{array}{r}6 \\
26.09\end{array}$ & $\begin{array}{r}6 \\
26.09\end{array}$ & $\begin{array}{r}9 \\
39.13\end{array}$ & 23 \\
\hline de02 a 03 & $\begin{array}{r}0 \\
0.00\end{array}$ & $\begin{array}{r}0 \\
0.00\end{array}$ & $\begin{array}{r}0 \\
0.00\end{array}$ & $\begin{array}{r}0 \\
0.00\end{array}$ & $\begin{array}{r}2 \\
50.00\end{array}$ & $\begin{array}{r}2 \\
50.00\end{array}$ & 4 \\
\hline de03a99 & $\begin{array}{r}1 \\
100.00\end{array}$ & $\begin{array}{r}0 \\
0.00\end{array}$ & $\begin{array}{r}0 \\
0.00\end{array}$ & $\begin{array}{r}0 \\
0.00\end{array}$ & $\begin{array}{r}0 \\
0.00\end{array}$ & $\begin{array}{r}0 \\
0.00\end{array}$ & 1 \\
\hline Total & 3 & 2 & 1 & 10 & 11 & 16 & 43 \\
\hline
\end{tabular}

TABLA LXIII: TIEMPO QUIRÚRGICO / TIEMPO DE INGRESO EN ENTEROCOLITIS

No existe ninguna relación estadísticamente significativa entre tiempo quirúrgico o tiempo total de quirófano con ninguna de las variables analizadas. 
RELACIONES ANTERIORMENTE ESTABLECIDAS EN LOS PACIENTES

DIAGNOSTICADOS DE DUCTUS ARTERIOSO PERSISTENTE:

Las siguientes tablas muestran las relaciones descritas con anterioridad pero sólo en los pacientes diagnosticados de Ductus Arterioso Persistente (DAP):

\begin{tabular}{|c|c|c|c|}
\hline \multicolumn{4}{|c|}{$\begin{array}{l}\text { Tabla de quir_horas_por MORTALIDAD } \\
\text { quir_horas_ } \\
{ }_{\text {MORTALIDAD (MORTALIDAD) }}\end{array}$} \\
\hline $\begin{array}{l}\text { Frecuencia } \\
\text { Pct fila }\end{array}$ & 0 & 1 & Total \\
\hline de00a01 & $\begin{array}{r}8 \\
88.89\end{array}$ & $\begin{array}{r}1 \\
11.11\end{array}$ & 9 \\
\hline de01a02 & $\begin{array}{r}23 \\
88.46\end{array}$ & $\begin{array}{r}3 \\
11.54\end{array}$ & 26 \\
\hline de02a03 & $\begin{array}{r}2 \\
66.67\end{array}$ & $\begin{array}{r}1 \\
33.33\end{array}$ & 3 \\
\hline de03a04 & $\begin{array}{r}2 \\
100.00\end{array}$ & $\begin{array}{r}0 \\
0.00\end{array}$ & 2 \\
\hline Total & 35 & 5 & 40 \\
\hline
\end{tabular}

TABLA LXIV: TIEMPO TOTAL DE QUIRÓFANO / MORTALIDAD EN DAP

\begin{tabular}{|c|c|c|c|}
\hline \multicolumn{4}{|c|}{$\begin{array}{c}\text { Tabla de quir_ho } \\
\text { quir_horas }\end{array}$} \\
\hline $\begin{array}{l}\text { Frecuencia } \\
\text { Pct fila }\end{array}$ & NO & SI & Total \\
\hline de00a01 & $\begin{array}{r}9 \\
100.00\end{array}$ & $\begin{array}{r}0 \\
0.00\end{array}$ & 9 \\
\hline de01a02 & $\begin{array}{r}23 \\
88.46\end{array}$ & $\begin{array}{r}3 \\
11.54\end{array}$ & 26 \\
\hline de02a03 & $\begin{array}{r}3 \\
100.00\end{array}$ & $\begin{array}{r}0 \\
0.00\end{array}$ & 3 \\
\hline de03a04 & $\begin{array}{r}2 \\
100.00\end{array}$ & $\begin{array}{r}0 \\
0.00\end{array}$ & 2 \\
\hline Total & 37 & 3 & 40 \\
\hline
\end{tabular}

TABLA LXV: TIEMPO TOTAL DE QUIRÓFANO / COMPLICACIONES EN DAP 


\begin{tabular}{|c|c|c|c|}
\hline \multicolumn{4}{|c|}{$\begin{array}{l}\text { Tabla de quir_horas_por REINTERVENCION } \\
\text { quir_horas_ } \\
{ }_{\text {REINTERVENCION (REINTERVENCION) }}\end{array}$} \\
\hline $\begin{array}{l}\text { Frecuencia } \\
\text { Pct fila }\end{array}$ & NO & SI & Total \\
\hline de00a01 & $\begin{array}{r}9 \\
100.00\end{array}$ & $\begin{array}{r}0 \\
0.00\end{array}$ & 9 \\
\hline de01a02 & $\begin{array}{r}25 \\
96.15\end{array}$ & $\begin{array}{r}1 \\
3.85\end{array}$ & 26 \\
\hline de02a03 & $\begin{array}{r}3 \\
100.00\end{array}$ & $\begin{array}{r}0 \\
0.00\end{array}$ & 3 \\
\hline de03a04 & $\begin{array}{r}2 \\
100.00\end{array}$ & $\begin{array}{r}0 \\
0.00\end{array}$ & 2 \\
\hline Total & 39 & 1 & 40 \\
\hline
\end{tabular}

TABLA LXVI: TIEMPO TOTAL DE QUIRÓFANO / REINTERVENCIÓN EN DAP

\begin{tabular}{|l|r|r|r|}
\hline \multicolumn{2}{|c|}{ Tabla de cir_horas_por MORTALIDAD } \\
cir_horas_ & MORTALIDAD (MORTALIDAD) \\
Frecuencia & 0 & 1 & Total \\
Pct fila & 0 & 2 & 22 \\
\hline de00 a 0.5 & 20 & 2 \\
& 90.91 & 9.09 & \\
\hline de00.5a01 & 12 & 2 & 14 \\
\hline de01 a 02 & 35.71 & 14.29 & \\
\hline Total & 35.00 & 25.00 & 4 \\
\hline
\end{tabular}

TABLA LXVII: TIEMPO QUIRÚRGICO / MORTALIDAD EN DAP

\begin{tabular}{|l|r|r|r|}
\hline \multicolumn{3}{|l|}{ Tabla de cir_horas_por complicaciones } \\
cir_horas_ \\
$\begin{array}{l}\text { Frecuencia } \\
\text { Pct fila }\end{array}$ & No & SI & Total \\
\hline de00 a 0.5 & 21 & 1 & 22 \\
& 95.45 & 4.55 & \\
\hline de00.5a01 & 12 & 2 & 14 \\
\hline de01 a 02 & 85.71 & 14.29 & \\
\hline Total & 100.00 & 0.00 & 4 \\
\end{tabular}

TABLA LXVIII: TIEMPO QUIRÚRGICO / COMPLICACIONES EN DAP 


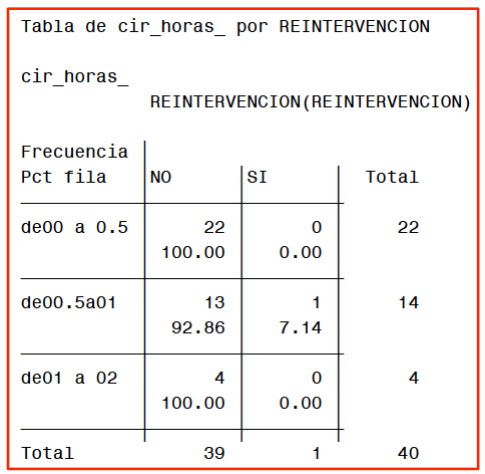

TABLA LXIX: TIEMPO QUIRÚRGICO / REINTERVENCIÓN EN DAP

\begin{tabular}{|c|c|c|c|c|c|c|c|}
\hline quir_horas_ & $\begin{array}{r}\mathrm{T} \\
\text { esta }\end{array}$ & $\begin{array}{l}\text { Tabla de } \\
\text { ancia_ }\end{array}$ & quir_horas & $s_{-}$por est & tancia_ & & \\
\hline $\begin{array}{l}\text { Frecuencia } \\
\text { Pct fila }\end{array}$ & $\left|\begin{array}{l}\text { de000a00 } \\
7\end{array}\right|$ & $\left|\begin{array}{l}\text { de008a01 } \\
4\end{array}\right|$ & $\left|\begin{array}{l}\text { de015a03 } \\
0\end{array}\right|$ & $\left|\begin{array}{l}\text { de031a06 } \\
0\end{array}\right|$ & $\mid \begin{array}{l}\text { de061a12 } \\
0\end{array}$ & $\left|\begin{array}{l}\text { de121a99 } \\
9\end{array}\right|$ & Total \\
\hline de00a01 & $\begin{array}{r}0 \\
0.00\end{array}$ & $\begin{array}{r}0 \\
0.00\end{array}$ & $\begin{array}{r}0 \\
0.00\end{array}$ & $\begin{array}{r}0 \\
0.00\end{array}$ & $\begin{array}{r}5 \\
55.56\end{array}$ & $\begin{array}{r}4 \\
44.44\end{array}$ & 9 \\
\hline de01a02 & $\begin{array}{r}1 \\
3.85\end{array}$ & $\begin{array}{r}1 \\
3.85\end{array}$ & $\begin{array}{r}0 \\
0.00\end{array}$ & $\begin{array}{r}3 \\
11.54\end{array}$ & $\begin{array}{r}12 \\
46.15\end{array}$ & $\begin{array}{r}9 \\
34.62\end{array}$ & 26 \\
\hline de02a03 & $\begin{array}{r}0 \\
0.00\end{array}$ & $\begin{array}{r}0 \\
0.00\end{array}$ & $\begin{array}{r}0 \\
0.00\end{array}$ & $\begin{array}{r}1 \\
33.33\end{array}$ & $\begin{array}{r}1 \\
33.33\end{array}$ & $\begin{array}{r}1 \\
33.33\end{array}$ & 3 \\
\hline de03a04 & $\begin{array}{r}0 \\
0.00\end{array}$ & $\begin{array}{r}0 \\
0.00\end{array}$ & $\begin{array}{r}1 \\
50.00\end{array}$ & $\begin{array}{r}0 \\
0.00\end{array}$ & $\begin{array}{r}1 \\
50.00\end{array}$ & $\begin{array}{r}0 \\
0.00\end{array}$ & 2 \\
\hline Total & 1 & 1 & 1 & 4 & 19 & 14 & 40 \\
\hline
\end{tabular}

TABLA LXX: TIEMPO TOTAL DE QUIRÓFANO/ TIEMPO DE INGRESO EN DAP

\begin{tabular}{|c|c|c|c|c|c|c|c|}
\hline $\begin{array}{l}\text { cir_noras_ } \\
\text { Frecuencia } \\
\text { Pct fila }\end{array}$ & $\begin{array}{l}\text { estanc } \\
\text { de000a00 } \\
7\end{array}$ & $\begin{array}{l}\text { Tabla de } \\
\text { cia }\end{array}$ & cir_horas & $\mid \begin{array}{l}\text { de } 031 \mathrm{a} 06 \\
0\end{array}$ & $\left|\begin{array}{l}\text { de061a12 } \\
0\end{array}\right|$ & $\mid \begin{array}{l}\text { de121a99 } \\
9\end{array}$ & Total \\
\hline de00 a 0.5 & $\begin{array}{r}0 \\
0.00\end{array}$ & $\begin{array}{r}0 \\
0.00\end{array}$ & $\begin{array}{r}0 \\
0.00\end{array}$ & $\begin{array}{r}1 \\
4.55\end{array}$ & $\begin{array}{r}9 \\
40.91\end{array}$ & $\begin{array}{r}12 \\
54.55\end{array}$ & 22 \\
\hline de00.5a01 & $\begin{array}{r}1 \\
7.14\end{array}$ & $\begin{array}{r}0 \\
0.00\end{array}$ & $\begin{array}{r}0 \\
0.00\end{array}$ & $\begin{array}{r}2 \\
14.29\end{array}$ & $\begin{array}{r}9 \\
64.29\end{array}$ & $\begin{array}{r}2 \\
14.29\end{array}$ & 14 \\
\hline de01 a 02 & $\begin{array}{r}0 \\
0.00\end{array}$ & $\begin{array}{r}1 \\
25.00\end{array}$ & $\begin{array}{r}1 \\
25.00\end{array}$ & $\begin{array}{r}1 \\
25.00\end{array}$ & $\begin{array}{r}1 \\
25.00\end{array}$ & $\begin{array}{r}0 \\
0.00\end{array}$ & 4 \\
\hline Total & 1 & 1 & 1 & 4 & 19 & 14 & 40 \\
\hline
\end{tabular}

TABLA LXXI: TIEMPO QUIRÚRGICO / TIEMPO DE INGRESO EN DAP 


\begin{tabular}{|c|c|c|c|c|c|c|c|}
\hline \multicolumn{8}{|c|}{ Tabla de quir_horas_por TIEMPO_UCIN_ } \\
\hline $\begin{array}{l}\text { Frecuencia } \\
\text { Pct fila }\end{array}$ & de00a01 & de02a03 & de04a07 & de07a14 & de15a30 & de31a99 & Total \\
\hline de00a01 & $\begin{array}{r}0 \\
0.00\end{array}$ & $\begin{array}{r}0 \\
0.00\end{array}$ & $\begin{array}{r}0 \\
0.00\end{array}$ & $\begin{array}{r}0 \\
0.00\end{array}$ & $\begin{array}{r}1 \\
11.11\end{array}$ & $\begin{array}{r}8 \\
88.89\end{array}$ & 9 \\
\hline de01a02 & $\begin{array}{r}1 \\
3.85\end{array}$ & $\begin{array}{r}1 \\
3.85\end{array}$ & $\begin{array}{r}1 \\
3.85\end{array}$ & $\begin{array}{r}1 \\
3.85\end{array}$ & $\begin{array}{r}4 \\
15.38\end{array}$ & $\begin{array}{r}18 \\
69.23\end{array}$ & 26 \\
\hline de02a03 & $\begin{array}{r}0 \\
0.00\end{array}$ & $\begin{array}{r}0 \\
0.00\end{array}$ & $\begin{array}{r}0 \\
0.00\end{array}$ & $\begin{array}{r}0 \\
0.00\end{array}$ & $\begin{array}{r}0 \\
0.00\end{array}$ & $\begin{array}{r}3 \\
100.00\end{array}$ & 3 \\
\hline de03a04 & $\begin{array}{r}0 \\
0.00\end{array}$ & $\begin{array}{r}1 \\
50.00\end{array}$ & $\begin{array}{r}0 \\
0.00\end{array}$ & $\begin{array}{r}1 \\
50.00\end{array}$ & $\begin{array}{r}0 \\
0.00\end{array}$ & $\begin{array}{r}0 \\
0.00\end{array}$ & 2 \\
\hline Total & 1 & 2 & 1 & 2 & 5 & 29 & 40 \\
\hline
\end{tabular}

TABLA LXXII: TIEMPO TOTAL DE QUIRÓFANO / TIEMPO DE INGRESO EN UCIN EN DAP

\begin{tabular}{|l|r|r|r|r|r|r|r|}
\hline \multicolumn{7}{|c|}{ Tabla de cir_horas_por TIEMPO_UCIN_ } \\
cir_horas_ \\
$\begin{array}{l}\text { Frecuencia } \\
\text { Pct fila }\end{array}$ & de00a01 & de02a03 & de04a07 & de07a14 & de15a30 & de31a99 & Total \\
\hline de00 a 0.5 & 0 & 0 & 1 & 0 & 3 & 18 & 22 \\
& 0.00 & 0.00 & 4.55 & 0.00 & 13.64 & 81.82 & \\
\hline de00.5a01 & 1 & 1 & 0 & 0 & 2 & 10 & 14 \\
\hline de01 a 02 & 0.14 & 7.14 & 0.00 & 0.00 & 14.29 & 71.43 & \\
\hline Total & 0.00 & 25.00 & 0.00 & 50.00 & 0.00 & 25.00 & \\
& 1 & 2 & 1 & 2 & 5 & 29 & 4 \\
\hline
\end{tabular}

TABLA LXXIII: TIEMPO QUIRÚRGICO / TIEMPO DE INGRESO EN UCIN EN DAP

Existe una relación estadísticamente significativa entre tiempo quirúrgico y tiempo de ingreso $\mathrm{P}=0.0099$ y tiempo de ingreso en UCIN 0.0473 y entre tiempo total de quirófano y tiempo de ingreso 0.0064 y tiempo de ingreso en UCIN 0.0325 
RELACIONES ANTERIORMENTE ESTABLECIDAS EN LOS PACIENTES

\section{INTERVENIDOS EN QUIRÓFANO (ANI):}

Las siguientes tablas muestran las relaciones descritas con anterioridad pero sólo en los pacientes intervenidos en quirófano (ANI):

\begin{tabular}{|c|c|c|c|}
\hline \multicolumn{4}{|c|}{$\begin{array}{l}\text { Tabla de quir_horas_por MORTALID/ } \\
\text { quir_horas__ } \\
{ }_{\text {MORTALIDAD(MORTALIDAD) }}\end{array}$} \\
\hline $\begin{array}{l}\text { Frecuencia } \\
\text { Pct Tila }\end{array}$ & 0 & 1 & Total \\
\hline de00a01 & $\begin{array}{r}4 \\
80.00\end{array}$ & $\begin{array}{r}1 \\
20.00\end{array}$ & 5 \\
\hline de01a02 & $\begin{array}{r}28 \\
90.32\end{array}$ & $\begin{array}{r}3 \\
9.68\end{array}$ & 31 \\
\hline de02a03 & $\begin{array}{r}35 \\
85.37\end{array}$ & $\begin{array}{r}6 \\
14.63\end{array}$ & 41 \\
\hline de03a04 & $\begin{array}{r}31 \\
77.50\end{array}$ & $\begin{array}{r}9 \\
22.50\end{array}$ & 40 \\
\hline de04a99 & $\begin{array}{r}28 \\
96.55\end{array}$ & $\begin{array}{r}1 \\
3.45\end{array}$ & 29 \\
\hline Total & 126 & 20 & 146 \\
\hline
\end{tabular}

TABLA LXXIV: TIEMPO TOTAL DE QUIRÓFANO / MORTALIDAD EN ANI

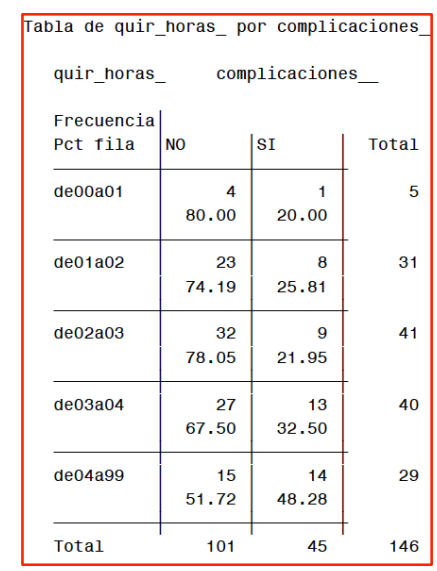

TABLA LXXV: TIEMPO TOTAL DE QUIRÓFANO / COMPLICACIONES EN ANI 


\begin{tabular}{|c|c|c|c|}
\hline \multicolumn{4}{|c|}{$\begin{array}{l}\text { Tabla de quir_horas_por REINTERVENCION } \\
\text { quir_horas__ } \\
\text { REINTERVENCION(REINTERVENCION) }\end{array}$} \\
\hline $\begin{array}{l}\text { Frecuencia } \\
\text { Pct fila }\end{array}$ & No & SI & Total \\
\hline de00a01 & $\begin{array}{r}4 \\
80.00\end{array}$ & $\begin{array}{r}1 \\
20.00\end{array}$ & 5 \\
\hline de $01 \mathrm{a} 02$ & $\begin{array}{r}26 \\
83.87\end{array}$ & $\begin{array}{r}5 \\
16.13\end{array}$ & 31 \\
\hline de02a03 & $\begin{array}{r}26 \\
63.41\end{array}$ & $\begin{array}{r}15 \\
36.59\end{array}$ & 41 \\
\hline de03a04 & $\begin{array}{r}26 \\
65.00\end{array}$ & $\begin{array}{r}14 \\
35.00\end{array}$ & 40 \\
\hline de04a99 & $\begin{array}{r}17 \\
58.62\end{array}$ & $\begin{array}{r}12 \\
41.38\end{array}$ & 29 \\
\hline Total & 99 & 47 & 146 \\
\hline
\end{tabular}

TABLA LXXVI: TIEMPO TOTAL DE QUIRÓFANO / REINTERVENCIÓN EN ANI

\begin{tabular}{|c|c|c|c|}
\hline $\begin{array}{l}\text { Tabla de } \\
\text { cir_horas_ }\end{array}$ & $\begin{array}{r}\text { ir_horas } \\
\text { MORTAL }\end{array}$ & $\begin{array}{l}\text { por MORT } \\
\text { DAD (MORT }\end{array}$ & $\begin{array}{l}\text { LIDAD } \\
\text { LIDAD) }\end{array}$ \\
\hline $\begin{array}{l}\text { Frecuencia } \\
\text { Pct fila }\end{array}$ & 이 & 1 & Total \\
\hline de00 a 0.5 & $\begin{array}{r}15 \\
83.33\end{array}$ & $\begin{array}{r}3 \\
16.67\end{array}$ & 18 \\
\hline de00.5a01 & $\begin{array}{r}20 \\
76.92\end{array}$ & $\begin{array}{r}6 \\
23.08\end{array}$ & 26 \\
\hline de01 a 02 & $\begin{array}{r}63 \\
88.73\end{array}$ & $\begin{array}{r}8 \\
11.27\end{array}$ & 71 \\
\hline de02 a 03 & $\begin{array}{r}17 \\
85.00\end{array}$ & $\begin{array}{r}3 \\
15.00\end{array}$ & 20 \\
\hline de03a99 & $\begin{array}{r}11 \\
100.00\end{array}$ & $\begin{array}{r}0 \\
0.00\end{array}$ & 11 \\
\hline Total & 126 & 20 & 146 \\
\hline
\end{tabular}

TABLA LXXVII: TIEMPO QUIRÚRGICO / MORTALIDAD EN ANI

\begin{tabular}{|c|c|c|c|}
\hline \multirow{2}{*}{$\begin{array}{l}\text { Tabla de cir } \\
\text { cir_horas_ } \\
\text { Frecuencia } \\
\text { Pct fila }\end{array}$} & \multicolumn{3}{|c|}{ complicaciones } \\
\hline & No & |SI & Total \\
\hline de00 a 0.5 & $\begin{array}{r}17 \\
94.44\end{array}$ & $\begin{array}{r}1 \\
5.56\end{array}$ & 18 \\
\hline de00.5a01 & $\begin{array}{r}18 \\
69.23\end{array}$ & $\begin{array}{r}8 \\
30.77\end{array}$ & 26 \\
\hline de01 a 02 & $\begin{array}{r}47 \\
66.20\end{array}$ & $\begin{array}{r}24 \\
33.80\end{array}$ & 71 \\
\hline de02 a 03 & $\begin{array}{r}15 \\
75.00\end{array}$ & $\begin{array}{r}5 \\
25.00\end{array}$ & 20 \\
\hline de03a99 & $\begin{array}{r}4 \\
36.36\end{array}$ & $\begin{array}{r}7 \\
63.64\end{array}$ & 11 \\
\hline Total & 101 & 45 & 146 \\
\hline
\end{tabular}

TABLA LXXVIII: TIEMPO QUIRÚRGICO / COMPLICACIONES EN ANI 


\begin{tabular}{|c|c|c|c|}
\hline \multirow{2}{*}{$\begin{array}{l}\text { Tabla de ci } \\
\text { cir_horas_ } \\
\text { Frecuencia } \\
\text { Pct fila }\end{array}$} & \multicolumn{3}{|c|}{ REINTERVENCION (REINTERVENCION) } \\
\hline & NO & SI & Total \\
\hline de00 a 0.5 & $\begin{array}{r}16 \\
88.89\end{array}$ & $\begin{array}{r}2 \\
11.11\end{array}$ & 18 \\
\hline de00.5a01 & $\begin{array}{r}18 \\
69.23\end{array}$ & $\begin{array}{r}8 \\
30.77\end{array}$ & 26 \\
\hline de01 a 02 & $\begin{array}{r}47 \\
66.20\end{array}$ & $\begin{array}{r}24 \\
33.80\end{array}$ & 71 \\
\hline de02 a 03 & $\begin{array}{r}13 \\
65.00\end{array}$ & $\begin{array}{r}7 \\
35.00\end{array}$ & 20 \\
\hline de03a99 & $\begin{array}{r}5 \\
45.45\end{array}$ & $\begin{array}{r}6 \\
54.55\end{array}$ & 11 \\
\hline Total & 99 & 47 & 146 \\
\hline
\end{tabular}

TABLA LXXIX: TIEMPO QUIRÚRGICO / REINTERVENCIÓN EN ANI

\begin{tabular}{|c|c|c|c|c|c|c|c|}
\hline quir_horas_ & $\begin{array}{r}\mathrm{T} \\
\text { esta }\end{array}$ & $\begin{array}{l}\text { Tabla de } \\
\text { ancia_ }\end{array}$ & quir_horas & $s_{-}$por est & tancia_ & & \\
\hline $\begin{array}{l}\text { Frecuencia } \\
\text { Pct fila }\end{array}$ & $\mid \begin{array}{l}\text { de000a00 } \\
7\end{array}$ & $\mid \begin{array}{l}\text { de008a01 } \\
4\end{array}$ & $\left|\begin{array}{l}\text { de015a03 } \\
0\end{array}\right|$ & $\left|\begin{array}{l}\text { de031a06 } \\
0\end{array}\right|$ & $\mid \begin{array}{l}\text { de061a12 } \\
0\end{array}$ & $\mid \begin{array}{l}\text { de121a99 } \\
9\end{array}$ & Total \\
\hline de00a01 & $\begin{array}{r}0 \\
0.00\end{array}$ & $\begin{array}{r}0 \\
0.00\end{array}$ & $\begin{array}{r}0 \\
0.00\end{array}$ & $\begin{array}{r}0 \\
0.00\end{array}$ & $\begin{array}{r}2 \\
40.00\end{array}$ & $\begin{array}{r}3 \\
60.00\end{array}$ & 5 \\
\hline de01a02 & $\begin{array}{r}2 \\
6.45\end{array}$ & $\begin{array}{r}3 \\
9.68\end{array}$ & $\begin{array}{r}4 \\
12.90\end{array}$ & $\begin{array}{r}3 \\
9.68\end{array}$ & $\begin{array}{r}11 \\
35.48\end{array}$ & $\begin{array}{r}8 \\
25.81\end{array}$ & 31 \\
\hline de02a03 & $\begin{array}{r}1 \\
2.44\end{array}$ & $\begin{array}{r}5 \\
12.20\end{array}$ & $\begin{array}{r}11 \\
26.83\end{array}$ & $\begin{array}{r}11 \\
26.83\end{array}$ & $\begin{array}{r}10 \\
24.39\end{array}$ & $\begin{array}{r}3 \\
7.32\end{array}$ & 41 \\
\hline de03a04 & $\begin{array}{r}0 \\
0.00\end{array}$ & $\begin{array}{r}8 \\
20.00\end{array}$ & $\begin{array}{r}11 \\
27.50\end{array}$ & $\begin{array}{r}7 \\
17.50\end{array}$ & $\begin{array}{r}9 \\
22.50\end{array}$ & $\begin{array}{r}5 \\
12.50\end{array}$ & 40 \\
\hline de04a99 & $\begin{array}{r}1 \\
3.45\end{array}$ & $\begin{array}{r}4 \\
13.79\end{array}$ & $\begin{array}{r}8 \\
27.59\end{array}$ & $\begin{array}{r}3 \\
10.34\end{array}$ & $\begin{array}{r}7 \\
24.14\end{array}$ & $\begin{array}{r}6 \\
20.69\end{array}$ & 29 \\
\hline Total & 4 & 20 & 34 & 24 & 39 & 25 & 146 \\
\hline
\end{tabular}

TABLA LXXX: TIEMPO TOTAL DE QUIRÓFANO / TIEMPO DE INGRESO EN ANI 


\begin{tabular}{|c|c|c|c|c|c|c|c|}
\hline $\begin{array}{l}\text { cir_noras_ } \\
\text { Frecuencia } \\
\text { Pct fila }\end{array}$ & $\begin{array}{l}\text { estanc } \\
\text { de000a00 } \\
7\end{array}$ & $\begin{array}{l}\text { Tabla de } \\
\text { cia_ } \\
\text { de008a01 } \\
4\end{array}$ & cir_horas & $\begin{array}{l}\text { de031a06 } \\
0\end{array}$ & $\mid \begin{array}{l}\text { de061a12 } \\
0\end{array}$ & $\left|\begin{array}{l}\text { de121a99 } \\
9\end{array}\right|$ & Total \\
\hline de00 a 0.5 & $\begin{array}{r}0 \\
0.00\end{array}$ & $\begin{array}{r}1 \\
5.56\end{array}$ & $\begin{array}{r}0 \\
0.00\end{array}$ & $\begin{array}{r}1 \\
5.56\end{array}$ & $\begin{array}{r}5 \\
27.78\end{array}$ & $\begin{array}{r}11 \\
61.11\end{array}$ & 18 \\
\hline de00.5a01 & $\begin{array}{r}2 \\
7.69\end{array}$ & $\begin{array}{r}3 \\
11.54\end{array}$ & $\begin{array}{r}7 \\
26.92\end{array}$ & $\begin{array}{r}4 \\
15.38\end{array}$ & $\begin{array}{r}9 \\
34.62\end{array}$ & $\begin{array}{r}1 \\
3.85\end{array}$ & 26 \\
\hline de01 a 02 & $\begin{array}{r}1 \\
1.41\end{array}$ & $\begin{array}{r}12 \\
16.90\end{array}$ & $\begin{array}{r}20 \\
28.17\end{array}$ & $\begin{array}{r}14 \\
19.72\end{array}$ & $\begin{array}{r}16 \\
22.54\end{array}$ & $\begin{array}{r}8 \\
11.27\end{array}$ & 71 \\
\hline de02 a 03 & $\begin{array}{r}1 \\
5.00\end{array}$ & $\begin{array}{r}3 \\
15.00\end{array}$ & $\begin{array}{r}4 \\
20.00\end{array}$ & $\begin{array}{r}4 \\
20.00\end{array}$ & $\begin{array}{r}4 \\
20.00\end{array}$ & $\begin{array}{r}4 \\
20.00\end{array}$ & 20 \\
\hline de03a99 & $\begin{array}{r}0 \\
0.00\end{array}$ & $\begin{array}{r}1 \\
9.09\end{array}$ & $\begin{array}{r}3 \\
27.27\end{array}$ & $\begin{array}{r}1 \\
9.09\end{array}$ & $\begin{array}{r}5 \\
45.45\end{array}$ & $\begin{array}{r}1 \\
9.09\end{array}$ & 11 \\
\hline Total & 4 & 20 & 34 & 24 & 39 & 25 & 146 \\
\hline
\end{tabular}

TABLA LXXXI: TIEMPO QUIRÚRGICO / TIEMPO DE INGRESO EN ANI

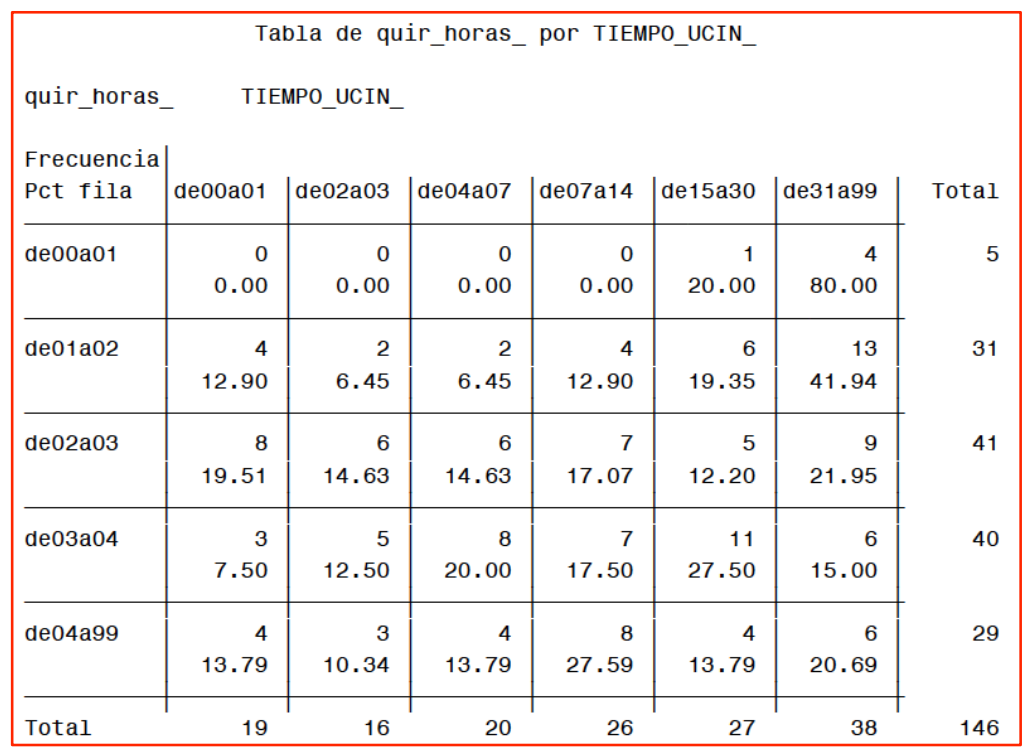

TABLA LXXXII: TIEMPO TOTAL DE QUIRÓFANO / TIEMPO DE INGRESO EN UCIN EN ANI 


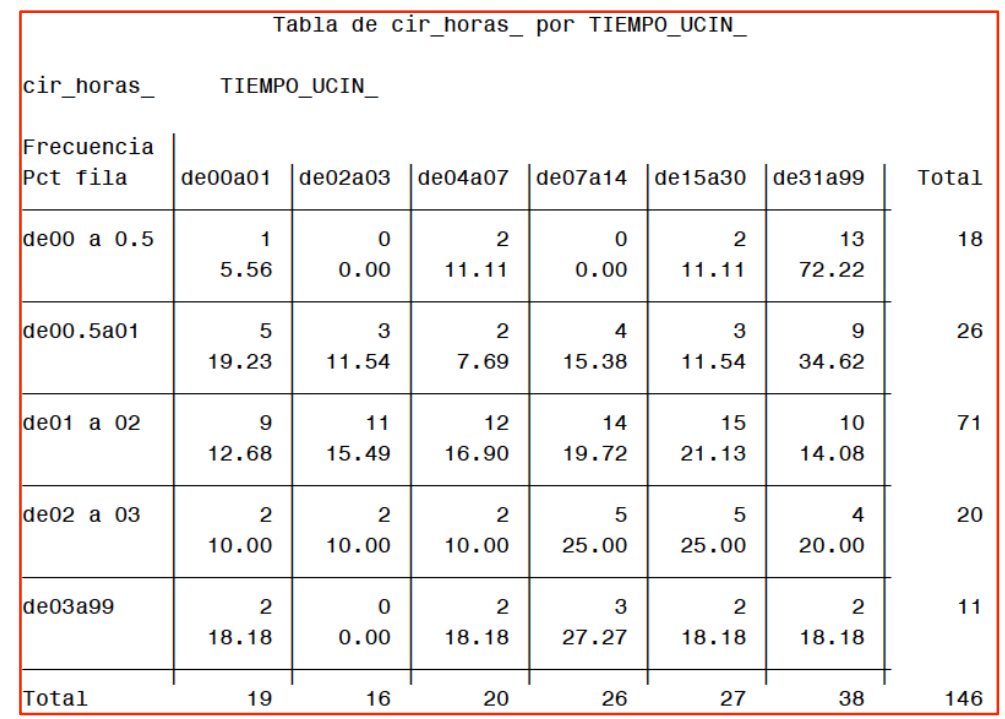

TABLA LXXXIII: TIEMPO QUIRÚRGICO / TIEMPO DE INGRESO EN UCIN EN ANI

Existe una relación estadísticamente significativa entre tiempo quirúrgico y complicaciones $\mathrm{P}=0.0247$, reintervención $\mathrm{P}=0.0224$ y tiempo de ingreso e UCIN $\mathrm{P}=0.0326$ y entre tiempo total de quirófano y complicaciones $\mathrm{P}=\mathbf{0 . 0 1 7 7}$, reintervención $\mathrm{P}=\mathbf{0 . 0 3 7 3}$ y tiempo de ingreso en UCIN 0.0125 
RELACIONES ANTERIORMENTE ESTABLECIDAS EN LOS PACIENTES

INTERVENIDOS EN LA UCIN:

Las siguientes tablas muestran las relaciones descritas con anterioridad pero sólo en los pacientes intervenidos en la Unidad de Cuidados Intensivos

Neonatales (UCIN):

\begin{tabular}{|c|c|c|c|}
\hline \multicolumn{4}{|c|}{$\begin{array}{l}\text { Tabla de quir_horas_por MORTALIDAD } \\
\text { quir_horas__} \\
{ }_{\text {MORTALIDAD (MORTALIDAD) }}\end{array}$} \\
\hline $\begin{array}{l}\text { Frecuencia } \\
\text { Pct Tila }\end{array}$ & 0 & 1 & Total \\
\hline de00a01 & $\begin{array}{r}5 \\
55.56\end{array}$ & $\begin{array}{r}4 \\
44.44\end{array}$ & 9 \\
\hline de $01 a 02$ & $\begin{array}{r}18 \\
69.23\end{array}$ & $\begin{array}{r}8 \\
30.77\end{array}$ & 26 \\
\hline de02a03 & $\begin{array}{r}7 \\
70.00\end{array}$ & $\begin{array}{r}3 \\
30.00\end{array}$ & 10 \\
\hline deоза04 & $\begin{array}{r}1 \\
50.00\end{array}$ & $\begin{array}{r}1 \\
50.00\end{array}$ & 2 \\
\hline de04a99 & $\begin{array}{r}0 \\
0.00\end{array}$ & $\begin{array}{r}1 \\
100.00\end{array}$ & 1 \\
\hline Total & 31 & 17 & 48 \\
\hline
\end{tabular}

TABLA LXXXIV: TIEMPO TOTAL DE QUIRÓFANO / MORTALIDAD DE UCIN

\begin{tabular}{|c|c|c|c|}
\hline \multicolumn{4}{|c|}{$\begin{array}{l}\text { Tabla de quir_horas_por complicaciones } \\
\text { quir_horas__ complicaciones_ }\end{array}$} \\
\hline $\begin{array}{l}\text { Frecuencia } \\
\text { Pct fila }\end{array}$ & NO & SI & Total \\
\hline de00a01 & $\begin{array}{r}5 \\
55.56\end{array}$ & $\begin{array}{r}4 \\
44.44\end{array}$ & 9 \\
\hline de01a02 & $\begin{array}{r}22 \\
84.62\end{array}$ & $\begin{array}{r}4 \\
15.38\end{array}$ & 26 \\
\hline de02a03 & $\begin{array}{r}5 \\
50.00\end{array}$ & $\begin{array}{r}5 \\
50.00\end{array}$ & 10 \\
\hline de03a04 & $\begin{array}{r}1 \\
50.00\end{array}$ & $\begin{array}{r}1 \\
50.00\end{array}$ & 2 \\
\hline de04a99 & $\begin{array}{r}1 \\
100.00\end{array}$ & $\begin{array}{r}0 \\
0.00\end{array}$ & 1 \\
\hline Total & 34 & 14 & 48 \\
\hline
\end{tabular}

TABLA LXXXV: TIEMPO TOTAL DE QUIRÓFANO / COMPLICACIONES DE UCIN 


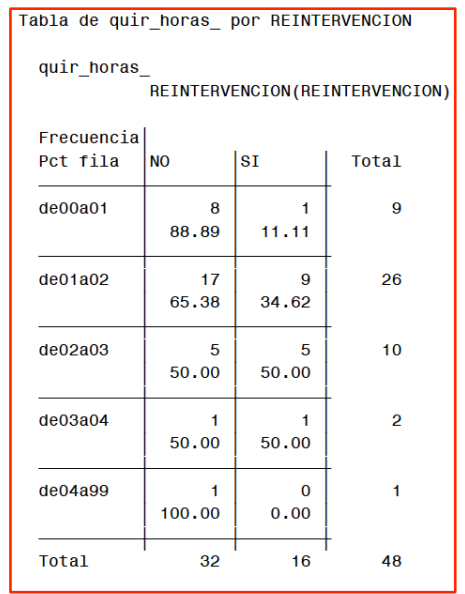

TABLA LXXXVI: TIEMPO TOTAL DE QUIRÓFANO / REINTERVENCIÓN DE UCIN

\begin{tabular}{|c|c|c|c|}
\hline \multirow{2}{*}{$\begin{array}{l}\quad \text { Tabla de } \\
\text { cir_horas_ } \\
\text { Frecuencia } \\
\text { Pct fila }\end{array}$} & $\begin{array}{r}\text { r_horas } \\
\text { MORTA }\end{array}$ & \multicolumn{2}{|c|}{ MORTAL IDAD (MORTALIDAD) } \\
\hline & 0 & 1 & Total \\
\hline de00 a 0.5 & $\begin{array}{r}9 \\
69.23\end{array}$ & $\begin{array}{r}4 \\
30.77\end{array}$ & 13 \\
\hline de00.5a01 & $\begin{array}{r}13 \\
68.42\end{array}$ & $\begin{array}{r}6 \\
31.58\end{array}$ & 19 \\
\hline de 01 a 02 & $\begin{array}{r}7 \\
58.33\end{array}$ & $\begin{array}{r}5 \\
41.67\end{array}$ & 12 \\
\hline de02 a 03 & $\begin{array}{r}2 \\
66.67\end{array}$ & $\begin{array}{r}1 \\
33.33\end{array}$ & 3 \\
\hline de03a99 & $\begin{array}{r}0 \\
0.00\end{array}$ & $\begin{array}{r}1 \\
100.00\end{array}$ & 1 \\
\hline & 31 & 17 & 48 \\
\hline
\end{tabular}

TABLA LXXXVII: TIEMPO QUIRÚRGICO / MORTALIDAD DE UCIN

\begin{tabular}{|c|c|c|c|}
\hline \multicolumn{4}{|c|}{$\begin{array}{l}\text { Tabla de cir_horas_por complicac } \\
\text { cir_horas__ complicaciones__ }\end{array}$} \\
\hline $\begin{array}{l}\text { Frecuencia } \\
\text { Pct fila }\end{array}$ & NO & SI & Total \\
\hline de 00 a 0.5 & $\begin{array}{r}10 \\
76.92\end{array}$ & $\begin{array}{r}3 \\
23.08\end{array}$ & 13 \\
\hline de $00.5 a 01$ & $\begin{array}{r}14 \\
73.68\end{array}$ & $\begin{array}{r}5 \\
26.32\end{array}$ & 19 \\
\hline de 01 a 02 & $\begin{array}{r}8 \\
66.67\end{array}$ & $\begin{array}{r}4 \\
33.33\end{array}$ & 12 \\
\hline de02 a 03 & $\begin{array}{r}1 \\
33.33\end{array}$ & $\begin{array}{r}2 \\
66.67\end{array}$ & 3 \\
\hline de03а99 & $\begin{array}{r}1 \\
100.00\end{array}$ & $\begin{array}{r}0 \\
0.00\end{array}$ & 1 \\
\hline Total & 34 & 14 & 48 \\
\hline
\end{tabular}

TABLA LXXXVIII: TIEMPO QUIRÚRGICO / COMPLICACIONES DE UCIN 


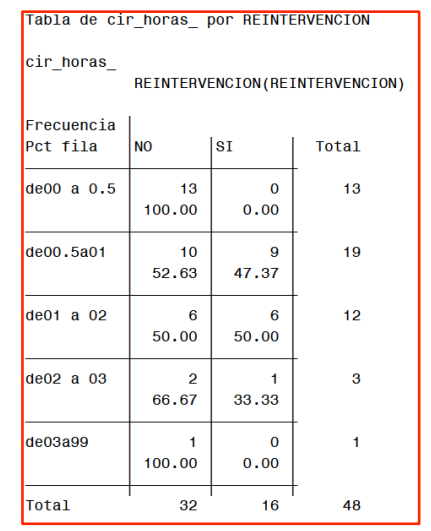

TABLA IXC: TIEMPO QUIRÚRGICO / REINTERVENCIÓN DE UCIN

\begin{tabular}{|c|c|c|c|c|c|c|c|}
\hline quir_horas & esta & $\begin{array}{l}\text { Tabla de } \\
\text { ancia_ }\end{array}$ & quir_horas & Is_ por est & tancia_ & & \\
\hline $\begin{array}{l}\text { Frecuencia } \\
\text { Pct fila }\end{array}$ & $\mid \begin{array}{l}\text { de000a00 } \\
7\end{array}$ & $\mid \begin{array}{l}\text { de008a01 } \\
4\end{array}$ & $\mid \begin{array}{l}\text { de015a03 } \\
0\end{array}$ & $\left|\begin{array}{l}\text { de031a06 } \\
0\end{array}\right|$ & de061a12 & $\mid \begin{array}{l}\text { de121a99 } \\
9\end{array}$ & Total \\
\hline de00a01 & $\begin{array}{r}2 \\
22.22\end{array}$ & $\begin{array}{r}1 \\
11.11\end{array}$ & $\begin{array}{r}0 \\
0.00\end{array}$ & $\begin{array}{r}1 \\
11.11\end{array}$ & $\begin{array}{r}4 \\
44.44\end{array}$ & $\begin{array}{r}1 \\
11.11\end{array}$ & 9 \\
\hline de01a02 & $\begin{array}{r}2 \\
7.69\end{array}$ & $\begin{array}{r}2 \\
7.69\end{array}$ & $\begin{array}{r}4 \\
15.38\end{array}$ & $\begin{array}{r}7 \\
26.92\end{array}$ & $\begin{array}{r}6 \\
23.08\end{array}$ & $\begin{array}{r}5 \\
19.23\end{array}$ & 26 \\
\hline de02a03 & $\begin{array}{r}0 \\
0.00\end{array}$ & $\begin{array}{r}0 \\
0.00\end{array}$ & $\begin{array}{r}3 \\
30.00\end{array}$ & $\begin{array}{r}0 \\
0.00\end{array}$ & $\begin{array}{r}3 \\
30.00\end{array}$ & $\begin{array}{r}4 \\
40.00\end{array}$ & 10 \\
\hline de03a04 & $\begin{array}{r}0 \\
0.00\end{array}$ & $\begin{array}{r}0 \\
0.00\end{array}$ & $\begin{array}{r}0 \\
0.00\end{array}$ & $\begin{array}{r}0 \\
0.00\end{array}$ & $\begin{array}{r}2 \\
100.00\end{array}$ & $\begin{array}{r}0 \\
0.00\end{array}$ & 2 \\
\hline de04a99 & $\begin{array}{r}0 \\
0.00\end{array}$ & $\begin{array}{r}0 \\
0.00\end{array}$ & $\begin{array}{r}0 \\
0.00\end{array}$ & $\begin{array}{r}1 \\
100.00\end{array}$ & $\begin{array}{r}0 \\
0.00\end{array}$ & $\begin{array}{r}0 \\
0.00\end{array}$ & 1 \\
\hline Total & 4 & 3 & 7 & 9 & 15 & 10 & 48 \\
\hline
\end{tabular}

TABLA XC: TIEMPO TOTAL DE QUIRÓFANO / TIEMPO DE INGRESO DE UCIN 


\begin{tabular}{|c|c|c|c|c|c|c|c|}
\hline $\begin{array}{l}\text { cir_horas_ } \\
\text { Frecuencia } \\
\text { Pct fila }\end{array}$ & $\begin{array}{l}\text { estanc } \\
\text { de000a00 } \\
7\end{array}$ & $\begin{array}{l}\text { Tabla de } \\
\text { cia_ } \\
\left.\right|_{4} ^{\text {de008a0 } 01}\end{array}$ & cir_horas & - por esta & $\left|\begin{array}{l}\text { de061a12 } \\
0\end{array}\right|$ & $\left|\begin{array}{l}\text { de121a99 } \\
9\end{array}\right|$ & Total \\
\hline de00 a 0.5 & $\begin{array}{r}2 \\
15.38\end{array}$ & $\begin{array}{r}1 \\
7.69\end{array}$ & $\begin{array}{r}1 \\
7.69\end{array}$ & $\begin{array}{r}1 \\
7.69\end{array}$ & $\begin{array}{r}5 \\
38.46\end{array}$ & $\begin{array}{r}3 \\
23.08\end{array}$ & 13 \\
\hline de00.5a01 & $\begin{array}{r}2 \\
10.53\end{array}$ & $\begin{array}{r}0 \\
0.00\end{array}$ & $\begin{array}{r}4 \\
21.05\end{array}$ & $\begin{array}{r}5 \\
26.32\end{array}$ & $\begin{array}{r}4 \\
21.05\end{array}$ & $\begin{array}{r}4 \\
21.05\end{array}$ & 19 \\
\hline de01 a 02 & $\begin{array}{r}0 \\
0.00\end{array}$ & $\begin{array}{r}2 \\
16.67\end{array}$ & $\begin{array}{r}1 \\
8.33\end{array}$ & $\begin{array}{r}2 \\
16.67\end{array}$ & $\begin{array}{r}5 \\
41.67\end{array}$ & $\begin{array}{r}2 \\
16.67\end{array}$ & 12 \\
\hline de02 a 03 & $\begin{array}{r}0 \\
0.00\end{array}$ & $\begin{array}{r}0 \\
0.00\end{array}$ & $\begin{array}{r}1 \\
33.33\end{array}$ & $\begin{array}{r}0 \\
0.00\end{array}$ & $\begin{array}{r}1 \\
33.33\end{array}$ & $\begin{array}{r}1 \\
33.33\end{array}$ & 3 \\
\hline de03a99 & $\begin{array}{r}0 \\
0.00\end{array}$ & $\begin{array}{r}0 \\
0.00\end{array}$ & $\begin{array}{r}0 \\
0.00\end{array}$ & $\begin{array}{r}1 \\
100.00\end{array}$ & $\begin{array}{r}0 \\
0.00\end{array}$ & $\begin{array}{r}0 \\
0.00\end{array}$ & 1 \\
\hline Total & 4 & 3 & 7 & 9 & 15 & 10 & 48 \\
\hline
\end{tabular}

TABLA XCI: TIEMPO QUIRÚRGICO TIEMPO DE INGRESO DE UCIN

\begin{tabular}{|c|c|c|c|c|c|c|c|}
\hline \multicolumn{8}{|c|}{ Tabla de quir_horas_por TIEMPO_UCIN_ } \\
\hline $\begin{array}{l}\text { Frecuencia } \\
\text { Pct fila }\end{array}$ & de00a01 & de02a03 & de04a07 & de07a14 & de15a30 & |de31a99 & Total \\
\hline de00a01 & $\begin{array}{r}1 \\
11.11\end{array}$ & $\begin{array}{r}0 \\
0.00\end{array}$ & $\begin{array}{r}0 \\
0.00\end{array}$ & $\begin{array}{r}2 \\
22.22\end{array}$ & $\begin{array}{r}1 \\
11.11\end{array}$ & $\begin{array}{r}5 \\
55.56\end{array}$ & 9 \\
\hline de01a02 & $\begin{array}{r}1 \\
3.85\end{array}$ & $\begin{array}{r}2 \\
7.69\end{array}$ & $\begin{array}{r}2 \\
7.69\end{array}$ & $\begin{array}{r}4 \\
15.38\end{array}$ & $\begin{array}{r}6 \\
23.08\end{array}$ & $\begin{array}{r}11 \\
42.31\end{array}$ & 26 \\
\hline de02a03 & $\begin{array}{r}0 \\
0.00\end{array}$ & $\begin{array}{r}0 \\
0.00\end{array}$ & $\begin{array}{r}0 \\
0.00\end{array}$ & $\begin{array}{r}1 \\
10.00\end{array}$ & $\begin{array}{r}2 \\
20.00\end{array}$ & $\begin{array}{r}7 \\
70.00\end{array}$ & 10 \\
\hline de03a04 & $\begin{array}{r}0 \\
0.00\end{array}$ & $\begin{array}{r}0 \\
0.00\end{array}$ & $\begin{array}{r}0 \\
0.00\end{array}$ & $\begin{array}{r}1 \\
50.00\end{array}$ & $\begin{array}{r}0 \\
0.00\end{array}$ & $\begin{array}{r}1 \\
50.00\end{array}$ & 2 \\
\hline de04a99 & $\begin{array}{r}0 \\
0.00\end{array}$ & $\begin{array}{r}0 \\
0.00\end{array}$ & $\begin{array}{r}0 \\
0.00\end{array}$ & $\begin{array}{r}0 \\
0.00\end{array}$ & $\begin{array}{r}0 \\
0.00\end{array}$ & $\begin{array}{r}1 \\
100.00\end{array}$ & 1 \\
\hline Total & 2 & 2 & 2 & 8 & 9 & 25 & 48 \\
\hline
\end{tabular}

TABLA XCII: TIEMPO TOTAL DE QUIRÓFANO / TIEMPO DE INGRESO EN UCIN DE UCIN 


\begin{tabular}{|c|c|c|c|c|c|c|c|}
\hline \multicolumn{8}{|c|}{ Tabla de cir_horas_por TIEMPO_UCIN_ } \\
\hline $\begin{array}{l}\text { Frecuencia } \\
\text { Pct fila }\end{array}$ & de00a01 & de02a03 & de04a07 & de07a14 & de15a30 & de31a99 & Total \\
\hline de00 a 0.5 & $\begin{array}{r}1 \\
7.69\end{array}$ & $\begin{array}{r}0 \\
0.00\end{array}$ & $\begin{array}{r}1 \\
7.69\end{array}$ & $\begin{array}{r}2 \\
15.38\end{array}$ & $\begin{array}{r}2 \\
15.38\end{array}$ & $\begin{array}{r}7 \\
53.85\end{array}$ & 13 \\
\hline de00.5a01 & $\begin{array}{r}1 \\
5.26\end{array}$ & $\begin{array}{r}2 \\
10.53\end{array}$ & $\begin{array}{r}1 \\
5.26\end{array}$ & $\begin{array}{r}2 \\
10.53\end{array}$ & $\begin{array}{r}6 \\
31.58\end{array}$ & $\begin{array}{r}7 \\
36.84\end{array}$ & 19 \\
\hline de01 a 02 & $\begin{array}{r}0 \\
0.00\end{array}$ & $\begin{array}{r}0 \\
0.00\end{array}$ & $\begin{array}{r}0 \\
0.00\end{array}$ & $\begin{array}{r}3 \\
25.00\end{array}$ & $\begin{array}{r}1 \\
8.33\end{array}$ & $\begin{array}{r}8 \\
66.67\end{array}$ & 12 \\
\hline de02 a 03 & $\begin{array}{r}0 \\
0.00\end{array}$ & $\begin{array}{r}0 \\
0.00\end{array}$ & $\begin{array}{r}0 \\
0.00\end{array}$ & $\begin{array}{r}1 \\
33.33\end{array}$ & $\begin{array}{r}0 \\
0.00\end{array}$ & $\begin{array}{r}2 \\
66.67\end{array}$ & 3 \\
\hline de03a99 & $\begin{array}{r}0 \\
0.00\end{array}$ & $\begin{array}{r}0 \\
0.00\end{array}$ & $\begin{array}{r}0 \\
0.00\end{array}$ & $\begin{array}{r}0 \\
0.00\end{array}$ & $\begin{array}{r}0 \\
0.00\end{array}$ & $\begin{array}{r}1 \\
100.00\end{array}$ & 1 \\
\hline Total & 2 & 2 & 2 & 8 & 9 & 25 & 48 \\
\hline
\end{tabular}

TABLA XCIII: TIEMPO QUIRÚRGICO / TIEMPO DE INGRESO EN UCIN DE UCIN

Existe una relación estadísticamente significativa entre tiempo

quirúrgico y reintervención $\mathrm{P}=0.0016$ y entre tiempo total de quirófano y reintervención $\mathrm{P}=\mathbf{0 . 0 4 4 1}$ 
DISCUSIÓN: 


\section{RELACIÓN ENTRE TIEMPO QUIRÚRGICO Y MORTALIDAD:}

De los resultados obtenidos en nuestro estudio no se puede deducir que exista una relación entre la duración del procedimiento quirúrgico y la mortalidad durante el ingreso en los recién nacidos que precisaron cirugía.

Estos resultados coinciden con los establecidos por Catré y sus colaboradores en su artículo sobre factores de riesgo de mortalidad precoz en neonatos intervenidos (46). En dicho estudio la población analizada tenía ciertas diferencias con la nuestra ya que el porcentaje de pacientes prematuros era menor y carecía de pacientes cardiópatas, ambos tipos de pacientes, por su características especiales podrían ser más sensibles a la hipotermia y las alteraciones metabólicas que conlleva. Por otra parte Catré distingue solo dos grupos en cuanto a la duración del procedimiento (duración mayor o menor a dos horas) mientras en nuestro estudio los intervalos temporales estudiados son muchos más.

A pesar de todo, los resultados son los mismos en ambas investigaciones. Parece por tanto que, en lo que a mortalidad se refiere, los mecanismos para el control de la temperatura y para revertir sus consecuencias metabólicas en caso de producirse son suficientes como para garantizar que una mayor duración del procedimiento no implique una mayor posibilidad de muerte. Lo mismo ocurre con los mecanismos para evitar la hemorragia o corregir sus consecuencias así como los dedicados a prevenir o tratar la infección que pueda estar relacionada con una prolongación de la cirugía.

Si analizamos en profundidad nuestros resultados veremos que existen pacientes cuya intervención dura poco tiempo y que sin embargo están asociados a una elevada mortalidad. Entre estos pacientes podemos destacar 
los prematuros con Enterocolitis Necrotizante que por su inestabilidad hemodinámica no pudieron recibir una laparotomía y recibieron solo un drenaje abdominal o aquellos pacientes en los que en una laparotomía exploradora se observó necrosis intestinal masiva sobre la que no se realizó tratamiento alguno. Este grupo de pacientes podría ser importante a la hora de explicar la falta de asociación entre tiempo quirúrgico y mortalidad.

\section{RELACIÓN ENTRE TIEMPO TOTAL DE QUIRÓFANO Y MORTALIDAD:}

En el artículo publicado por Parente y Colaboradores (73) en pacientes de este mismo Hospital que compara los pacientes intervenidos en La Unidad de Cuidados Intensivos Neonatales y los intervenidos en quirófano se observa que hay una mayor pérdida de temperatura en estos últimos lo cual no es sólo debido a la intervención en sí , también contribuye el traslado y el tiempo que el paciente pasa en quirófano antes y después de la cirugía. Por ello decidimos en este estudio incluir la variable tiempo total de quirófano como posible factor de riesgo de mortalidad postquirúrgica en recién nacidos.

Sin embargo los resultados obtenidos tampoco permiten establecer una relación entre ambos.

El razonamiento es muy similar al del punto anterior, los mecanismos para el control de temperatura y para corregir las alteraciones metabólicas que provoca la hipotermia han logrado que la mortalidad no aumente a pesar de un tiempo total de quirófano prolongado.

La razón por la cual la relación entre tiempo total de quirófano y mortalidad esté mas próxima a la significación estadística que la del tiempo quirúrgico es posiblemente que los pacientes más graves suelen precisar mayores medidas 
preoperatorias de monitorización y un período de estabilización tras la cirugía más prolongado lo cual alarga el tiempo total de quirófano sin alargar el tiempo quirúrgico.

No hemos encontrado ningún estudio que mencione el tiempo total de quirófano como posible factor de riesgo de mortalidad en cirugía neonatal que corrobore o rebata nuestros resultados.

\section{RELACIÓN ENTRE TIEMPO QUIRÚRGICO Y TIEMPO DE INGRESO:}

Los resultados obtenidos no permiten establecer una asociación entre tiempo quirúrgico y tiempo de ingreso en los recién nacidos intervenidos.

Para explicar estos resultados es importante tener en cuenta que el tiempo medio de ingreso de estos pacientes es muy largo. Esto hace que el tiempo necesario para corregir la hipotermia y sus alteraciones metabólicas, compensar la anemia secundaria a un posible sangrado o tratar una infección del lecho quirúrgico o de la herida no repercuta en la estancia hospitalaria total.

A todo ello puede contribuir el hecho de que una gran proporción de los pacientes de nuestro estudio son grandes prematuros intervenidos por Enterocolitis Necrotizante o Ductus Arterioso Persistente. Este tipo de pacientes presentan otras patologías asociadas como la Enfermedad por Membrana Hialina o hemorragias cerebrales que prolongan mucho su tiempo de ingreso y que nada tienen que ver con la cirugía.

Por otra parte hay procedimientos cuya duración es corta y que asocian tiempos de ingreso prolongados como por ejemplo las mediciones de cabos de las atresias de esófago “ long gap” ya que el paciente permanece ingresado por 
lo general hasta realizarse el tratamiento definitivo lo que puede llevar varios meses.

\section{RELACIÓN ENTRE TIEMPO TOTAL DE QUIRÓFANO Y TIEMPO DE}

\section{INGRESO:}

Al igual que en el caso anterior tampoco se puede concluir que haya una asociación entre tiempo total de quirófano y estancia hospitalaria.

El razonamiento para explicar este resultado es exactamente el mismo: por un lado la estancia media prolongada y por otro la existencia de procedimientos cortos que requieren estancias muy prolongadas.

Por otra parte al igual que ocurre con la mortalidad, la relación del tiempo total de quirófano y el tiempo de ingreso está mas próxima a la significación estadística que la del tiempo quirúrgico estricto y esto puede deberse como ya comentamos antes a que los pacientes más graves suelen requerir una monitorización más laboriosa y una estabilización más prolongada tras la cirugía.

\section{RELACIÓN ENTRE TIEMPO QUIRÚRGICO Y TIEMPO DE INGRESO EN}

\section{UCIN:}

Según los resultados obtenidos en nuestro estudio existe una relación entre una mayor duración de la cirugía y una estancia más prolongada en la Unidad de Cuidados Intensivos Neonatales. Este hallazgo es importante ya que demuestra que la recuperación del paciente tras un procedimiento quirúrgico largo es más prolongada seguramente por la combinación de varios factores como es el alcanzar el equilibrio térmico y metabólico tras la cirugía y 
recuperarse de la pérdida hemática por un lado o reponerse de una posible complicación o reintervención por otro.

Además existe una implicación importante de este punto en el gasto sanitario.

De hecho las unidades de cuidados intensivos neonatales figuran entre las secciones más caras de un hospital. Reducir el tiempo de cirugía podría ayudar a recortar gran parte del presupuesto que consume este tipo de pacientes $(95,96,97,98)$.

Por otra parte un tiempo más largo de ingreso en esta unidad supone un mayor riesgo de infecciones por patógenos resistentes a antibióticos así como una menor interrelación del paciente con su madre lo cual es de vital importancia para el bienestar del niño.

\section{RELACIÓN ENTRE TIEMPO TOTAL DE QUIRÓFANO Y TIEMPO DE}

\section{INGRESO EN UCIN:}

Al igual que en el caso anterior un mayor tiempo total de quirófano está relacionado con un mayor tiempo de ingreso en UCN. Se podría pensar que esto es debido a que una gran proporción del tiempo total de quirófano se corresponde con el tiempo quirúrgico sin embargo el hecho de que la asociación entre tiempo total de quirófano y tiempo de ingreso en UCIN sea más fuerte que la existente entre este último y el tiempo quirúrgico nos hace pensar que el tiempo extra que el paciente pasa en quirófano antes y después de la cirugía también resulta de vital importancia tanto por su influencia sobre la hipotermia como por el hecho de que los pacientes en los que el tiempo pre y postoperatorio que pasan en quirófano es más largo son los pacientes más inestables. Y por tanto más graves. 


\section{RELACIÓN ENTRE TIEMPO QUIRÚRGICO Y COMPLICACIONES:}

Los resultados demuestran una asociación entre un tiempo quirúrgico más largo y una mayor frecuencia de complicaciones.

Existe una discrepancia entre nuestro estudio y el de Catre et cols (45). sobre factores de riesgo relacionados con complicaciones mayores en cirugía neonatal. En su estudio Catre divide los pacientes en 2 grupos según la cirugía dure más o menos de 2 horas. Por otro lado podemos deducir por los diagnósticos de los pacientes intervenidos que la proporción de pacientes prematuros es menor, además carece de pacientes cardiópatas, todo ello hace que su población sea menos compleja que la nuestra como se observa también si comparamos la mortalidad. Teniendo en cuenta que los pacientes más complejos son más susceptibles a estas complicaciones esto podría explicar las discrepancias.

La relación entre un mayor tiempo quirúrgico y una mayor morbilidad puede deberse a varias causas. Por un lado las alteraciones metabólicas secundarias a la hipotermia y la pérdida hemática vuelven al paciente más sensible a las complicaciones. Por otro lado una mayor duración de la cirugía supone un mayor tiempo de exposición del área quirúrgica a los gérmenes patógenos y está relacionada con un mayor riesgo de infección. Finalmente en los pacientes con meconio o heces en la cavidad abdominal un mayor tiempo de exposición a los mismos podría facilitar la creación de bridas y las obstrucciones intestinales.

Por lo tanto reduciendo el tiempo quirúrgico de una intervención podríamos reducir el porcentaje de complicaciones que por otra parte ,como ya hemos 
visto, incrementarían el tiempo de estancia en UCIN y el gasto sanitario así como el sufrimiento del recién nacido.

\section{RELACIÓN ENTRE TIEMPO TOTAL DE QUIRÓFANO Y COMPLICACIONES:}

Los resultados demuestran que un mayor tiempo total de quirófano está relacionado con una mayor frecuencia de complicaciones. Sin embargo en este caso la asociación entre tiempo total de quirófano y complicaciones es menos fuerte que la establecida entre estas y el tiempo quirúrgico lo cual nos lleva a pensar que en este caso es el componente del tiempo intraoperatorio el que más contribuye a generar morbilidad por el riesgo más elevado de infección fundamentalmente.

\section{RELACIÓN ENTRE TIEMPO QUIRÚRGICO Y REINTERVENCIÓN:}

Los resultados obtenidos muestran que existe una relación entre tiempo quirúrgico y posibilidad de reintervención. Cuanto más dura la cirugía más posibilidades hay de que el paciente requiera un segundo procedimiento. No hemos encontrado ninguna publicación que estudie esta relación que a nosotros nos parece importante para determinar la importancia del tiempo quirúrgico como factor de riesgo.

Dicha relación podría explicarse por varios factores: En primer lugar si una mayor duración de la cirugía está relacionada con una mayor tasa de complicaciones y muchas de estas requieren tratamiento quirúrgico podemos deducir que a mayor tiempo quirúrgico mayor riesgo de reintervención. Por otra parte existen reintervenciones no relacionadas con las complicaciones como por ejemplo una reconstrucción precoz del tránsito para mejorar la 
ganancia ponderal y este tipo de reintervenciones suelen estar relacionadas con un síndrome de intestino corto que a su vez se relaciona con resecciones intestinales extensas y por tanto con cirugías largas.

Las reintervenciones suponen un hándicap para el paciente tanto por el dolor como por el tiempo de ayuno así como por el hecho de que volver a precisar ventilación mecánica y oxígeno retrasa la recuperación del recién nacido desde el punto de vista respiratorio. Por otra parte aumentan el tiempo de ingreso en UCIN con los inconvenientes ya mencionados que esto acarrea. Finalmente Catre en su artículo sobre complicaciones postoperatorias en cirugía neonatal relaciona las reintervenciones con una mayor morbilidad (45).

Por todo ello reducir el tiempo quirúrgico podría suponer un gran beneficio para el paciente al evitar un mayor riesgo de reintervención.

\section{RELACIÓN ENTRE TIEMPO TOTAL DE QUIRÓFANO Y REINTERVENCIÓN}

Al igual que ocurre con las complicaciones existe una relación entre un mayor tiempo total de quirófano y un mayor riesgo de reintervención. Sin embargo por las mismas razones que en el caso de las complicaciones creemos que esta relación depende más de la duración de la cirugía que del tiempo preoperatorio o postoperatorio que el paciente pasa en quirófano.

\section{ANÁLISIS DE LOS PACIENTES SOMETIDOS A LAPAROTOMÍA}

Si repetimos los análisis anteriores sólo en los pacientes sometidos a laparotomía tenemos un grupo más pequeño de pacientes y por tanto más dificultades para alcanzar significación estadística. A pesar de todo ello observamos que en este grupo de pacientes existe una relación entre la 
duración de la cirugía y la mortalidad. Por lo tanto el riesgo de una cirugía más larga repercute mucho más en este grupo de pacientes. Fuera del riesgo de hipotermia lo que más caracteriza a los pacientes laparotomizados con respecto a los demás grupos es probablemente el riesgo de infección. Muchos de estos niños presentan perforaciones intestinales previas a la cirugía o exposición del campo quirúrgico a las heces del paciente durante el procedimiento, si esto se prolonga en el tiempo en un paciente ya de por sí más sensible a la infección puede provocar un shock séptico de difícil manejo. Por otra parte en estos niños también se observa una relación entre el tiempo quirúrgico y el tiempo total de ingreso lo que tiene una gran importancia en el gasto sanitario.

Estas relaciones probablemente se vean beneficiadas por el hecho de que al analizar sólo los pacientes laparotomizados evitamos pacientes con alta mortalidad y tiempos quirúrgicos muy cortos, los drenajes abdominales y otros con tiempos cortos y largos períodos de ingreso como son las mediciones de cabos.

En cuanto al tiempo total de quirófano su influencia en la mortalidad también es importante, no sólo por la duración de la cirugía. Probablemente un paciente inestable tras un procedimiento largo precise un mayor tiempo de estabilización lo cual explique también que un tiempo total de quirófano prolongado se relacione con una mayor mortalidad.

\section{ANÁLISIS DE LOS PACIENTES SOMETIDOS A TORACOTOMÍAS:}

Al igual que en el caso anterior al reducir el número de pacientes encontramos más dificultades para encontrar significación estadística. Si embargo hallamos 
una relación entre tiempo quirúrgico y reintervención y tiempo de ingreso en UCIN y casi en las complicaciones $(\mathrm{P}=0.052)$ lo cual es muy similar al análisis en la totalidad de pacientes. La diferencia está en que a pesar de ser un grupo menor sí encontramos asociación entre tiempo quirúrgico y tiempo total de ingreso. En esto último puede influir el hecho de que gran parte de las toracotomías son para tratar Ductus Arterioso Persistente y siendo el Hospital Gregorio Marañón centro de referencia para cirugía cardiaca muchos de estos pacientes sólo permanecen en el hospital hasta que se recuperan de la cirugía y se comprueba la efectividad de la misma siendo posteriormente trasladados a su centro de origen. Al contrario que en las laparotomías no se halla relación con la mortalidad posiblemente porque las toracotomías son cirugías más limpias y con menor riesgo de infección generalizada y sepsis.

En cuanto al tiempo total de quirófano las relaciones que se establecen son las mismas más la relación con las complicaciones que en este caso si que es significativa lo cual nos ratifica en que no sólo influye en el pronóstico la duración de la cirugía en sí y que el tiempo que el paciente pasa en quirófano hasta que empieza el procedimiento o una vez que acaba el mismo también es de gran importancia para el pronóstico, bien por la hipotermia , bien por el hecho de que los pacientes más críticos precisan más procedimientos por parte del anestesista.

\section{ANÁLISIS DE LOS PACIENTES CON ENTEROCOLITIS NECROTIZANTE:}

En el caso de las enterocolitis necrotizantes no existe asociación entre tiempo quirúrgico o tiempo total de quirófano con ninguna de las variables analizadas. 
¿Cómo es posible esto si los pacientes prematuros deberían ser los más sensibles a la hipotermia, la infección o el sangrado?. En primer lugar al reducir el tamaño de la muestra es más difícil encontrar significación. Sin embargo hay otros factores que influyen de gran manera en el resultado.

Es importante tener en cuenta los pacientes que han sido sometidos a un drenaje abdominal, se trata de pacientes respiratoriamente y hemodinámicamente inestables que no soportan una intervención tan agresiva como la laparotomía y son pacientes con una muy elevada mortalidad. Por otra parte el tiempo quirúrgico de esta intervención es muy corto por lo que a la hora de establecer la relación entre tiempo quirúrgico y mortalidad penalizan mucho el resultado final. Lo mismo ocurre con los pacientes que se sometieron a una laparotomía y en los que se halló una necrosis intestinal masiva. En estos pacientes no suele realizarse resección intestinal y el tiempo quirúrgico se acorta y sin embargo casi todos fallecen. Además tanto los primeros como los segundos suelen ser sometidos a una laparotomía "second look" si sobreviven por lo que también penalizan a la hora de valorar una reintervención. Finalmente en los pacientes pretérmino existen otras muchas causas de mortalidad a parte de la enterocolitis necrotizante como son los problemas respiratorios, hemodinámicos o el riesgo de hemorragia cerebral. En cuanto al tiempo de ingreso total y el tiempo de ingreso en UCIN los pacientes prematuros tienen ingresos muy prolongados por lo que en ellos la recuperación postoperatoria más o menos prolongada influye menos que en otros pacientes con ingresos más cortos. Por otro lado la comorbilidad del recién nacido prematuro es tan elevada que existen muchas causas que pueden 
influir más en el tiempo de ingreso total y en la UCIN que la duración de la cirugía.

\section{ANÁLISIS DE LOS PACIENTES CON DUCTUS ARTERIOSO PERSISTENTE:}

En este grupo de pacientes, también prematuros si hallamos una relación entre el tiempo quirúrgico y el tiempo total de quirófano con el tiempo de ingreso total y el tiempo de ingreso en UCIN.

Sin embargo no se halla relación con las complicaciones ni con la reintervención. Esto seguramente es debido a la baja tasa de complicaciones y reintervenciones en este grupo de pacientes seguramente por el hecho de tratarse de una cirugía limpia y, por tanto, un riesgo infeccioso muy bajo. Sorprende el hecho de que el tiempo quirúrgico influya en este grupo sobre la estancia hospitalaria total. Uno de los factores que pueden influir sobre este hecho es que , al ser centro de referencia de patología cardiovascular pediátrica el Gregorio Marañón recibe pacientes prematuros de otros centros para intervenirse del Ductus Arterioso Persistente y que estos pacientes son trasladados de nuevo a su centro de origen una vez que se recuperan de la intervención y se comprueba que esta ha sido efectiva. Por otra parte los pacientes más complejos son posiblemente los que se intervinieron de una enterocolitis y posteriormente del Ductus y estos pacientes en nuestro estudio figuran dentro de las enterocolitis por ser aquella la primera intervención.

\section{ANÁLISIS DE LOS PACIENTES INTERVENIDOS EN QUIRÓFANO:}

Al igual que en la totalidad de los pacientes intervenidos, si estudiamos sólo a los pacientes intervenidos en quirófano (ANI) comprobamos que existe una 
asociación entre tiempo quirúrgico y tiempo total de quirófano prolongados y una mayor frecuencia de complicaciones, reintervenciones y tiempo de ingreso en UCIN siendo las razones superponibles a las ya mencionadas en los puntos $5,6,7,8,9$ y 10 . Sin embargo nos interesa ver si existe alguna diferencia entre este grupo de pacientes y los intervenidos en la UCIN por varias razones: En primer lugar como se expone en el estudio realizado por Parente y cols.(73) en una población superponible a la de este estudio pues se realizó en el mismo hospital y en intervalos temporales próximos, existe una diferencia de temperatura entre los pacientes intervenidos en quirófano y los intervenidos en la Unidad de Cuidados Intensivos sufriendo una mayor hipotermia los primeros. En segundo lugar los pacientes intervenidos en la UCIN son pacientes más graves, inestables hemodinámicamente y respiratoriamente y que no pueden ser trasladados por ello al quirófano.

\section{ANÁLISIS DE LOS PACIENTES INTERVENIDOS EN LA UCIN:}

A pesar de ser pacientes más graves, en los neonatos intervenidos en la UCIN el tiempo quirúrgico y el tiempo total de quirófano influyen menos en las complicaciones y el tiempo de ingreso en UCIN que en los pacientes intervenidos en quirófano. Sin embargo relacionar estos resultados sólo con el hecho de que sufren menos hipotermia es un poco prematuro. Hay que tener en cuenta que el grupo de pacientes intervenidos en la UCIN es sensiblemente menor por lo que es más difícil encontrar diferencias estadísticamentes significativas. Por otra parte dentro de este grupo se encuentran los pacientes más graves y con mayor comorbilidad así como los pacientes que se sometieron a cirugías cortas pero con peor evolución como son los drenajes 
abdominales. Por ello no podemos asegurar que sea preferible intervenir al recién nacido en la Unidad de Cuidados Intensivos Neonatales pero al tener menos riesgo de hipotermia es posible que el tiempo quirúrgico influya menos en el tiempo que estos pacientes pasan en la UCIN o en la posibilidad de complicarse. Sin embargo sí existe una relación entre tiempo quirúrgico y reintervención. En ello puede influir el factor de las resecciones intestinales largas del que hablamos previamente en el punto 9 o también si una gastrosquisis intervenida en la UCIN precisó silo, cirugía mas larga que el cierre primario y que requiere siempre una reintervención para la retirada del mismo. 


\section{CONCLUSIONES:}


A tenor de los resultados obtenidos en este estudio podemos concluir que:

- El tiempo quirúrgico es un factor de riesgo importante que influye en la evolución de los recién nacidos que van a ser sometidos a una cirugía mayor:

- Aunque no influye en la mortalidad global sí lo hace en grupos concretos como los pacientes que se someten a laparotomías.

- Un tiempo quirúrgico prolongado está relacionado con una mayor frecuencia de complicaciones y reintervenciones.

- En general no determina un mayor tiempo de ingreso pero sí lo hace en grupos concretos como son los pacientes diagnosticados de Ductus Arterioso Persistente y los que se someten a una laparotomía o toracotomía.

- Influye en gran manera sobre el tiempo de ingreso en la Unidad de Cuidados Intensivos Neonatales lo que puede tener una gran repercusión en el gasto sanitario

\section{- El tiempo total de quirófano también puede considerarse factor de} riesgo en estos pacientes:

- Tampoco influye en la mortalidad global pero sí en los pacientes laparotomizados

- Se relaciona con una mayor posibilidad de complicaciones y reintervenciones aunque parece ser que en menor medida que el anterior

- Tampoco determina un mayor tiempo de ingreso en general pero sí en grupos concretos como los pacientes diagnosticados 
de Ductus Arterioso Persistente y los que recibieron toracotomías

- Su influencia sobre el tiempo de ingreso en la Unidad de Cuidados Intensivos Neonatales es incluso mayor que la del tiempo quirúrgico.

- En los pacientes con Enterocolitis Necrotizante el tiempo quirúrgico y el tiempo total de quirófano no pueden considerarse como factor de riesgo: posiblemente debido a la alta mortalidad de los pacientes que recibieron drenaje abdominal y a la elevada comorbilidad y largo tiempo de ingreso.

- Las conclusiones generales son asimilables al grupo de pacientes intervenidos en quirófano

- En los pacientes intervenidos en la Unidad de Cuidados Intensivos Neonatales la influencia del tiempo quirúrgico sobre las complicaciones y el tiempo de ingreso en UCIN es menor. Si bien serían necesarios más estudios para confirmar la influencia de la hipotermia en ello. 


\section{BIBLIOGRAFÍA:}


- 1. Zachariou Z. Pediatric Surgery Digest. Springer-Verlag Berlin Heidelberg 2009.

- 2. Ziegler MM, Azizkhan RG, Weber TR. Operative Pediatric Surgery. McGraw-Hill. 2003.

- 3. Puri P. Pediatric Surgery. Springer-Verlag Berlin Heidelberg New York 2006.

- 4. O"Neill JA, Rowe MI, Grosfeld JL, Fonkalsrud EW, Coran AG. Pediatric Surgery 5th edition. Mosby 1998

- 5. Cruz M, Brines J, Carrascosa A, Crespo M, Jímenez R, Molina JA. Manual de Pediatría tercera edición. Majadahonda (Madrid) 2013.

- 6. Navascues JA, Vazquez J. Manual de asistencia al trauma pediátrico. Segunda edición. Madrid Septiembre2001.

- 7. Problemas quirúrgicos del prematuro. Texto del Master en Neonatología de la Sociedad Española de Neonatología. Bloque 1. Asistencia al recién nacido prematuro. 4-25. Pavon Delgado A, Suarez Delgado JM Editorial Panamericana. 2015.

- 8. Aschraft Murphy, Sharp, Sigalet, Snyder.Cirugía Pediátrica. Mcgraw Hill. $3^{\text {a }}$ Edición.

- 9. Sarquis AF, Miyaki M, Monica NL. CRIB score for predicting neonatal mortality risk. J Pediatr (Rio J) 2002; 78 (3): 225-9

- 10.Boat AC, Sadhasivam S, Loepke AW, Kurth CD. Outcome for the extremely premature neonate: how far do we push the edge? Pediatr Anesth 2011; 21(7): 765-70. 
- 11.Boyle EM, Poulsen G, Field DJ, Kurinczuk JJ, Wolke D, Alfirevic Z, et al. Effects of gestational age at birth on health outcomes at 3 and 5 years of age: population based cohort study. BMJ 2012; 344: e896.

- $\quad$ 12.Muldoon KA, Galway LP, Nakajima M, Kanters S, Hogg RS, Bendavid E, et al. Health system determinants of infant, child and maternal mortality: A cross-sectional study of UN member countries. Global Health 2011; 7: 42.

- 13.Lee HC, Subeh M, Gould JB. Low Apgar score and mortality in extremely preterm neonates born in the United States. Acta Paediatr 2010; 99(12): 1785-9.

- $\quad$ 14.International Neonatal Network. The CRIB (Clinical Risk Index for Babies) Score: a tool for assessing initial neonatal risk and comparing performance of neonatal intensive-care units. Lancet 1993;342:193-8.

- $\quad$ 15.Parry G, Tucker J, Tarnow-Mordi W, UK Neonatal Staffing Study Collaborative Group. CRIB II: an update of the Clinical Risk Index for Babies score. Lancet 2003;361: 1789-91.

- 16.Richardson DK, Gray JE, McKormick MC, Workman K, Goldmann DA. Score for Neonatal Acute Physiology: a physiological severity index for neonatal intensive care. Pediatrics 1993;91:617-23.

- 17.Richardson DK, Phibbs CS, Gray JE, McKormick MC, Workman-Daniels K, Goldmann DA. Birth weight and illness severity: independent predictors of neonatal mortality. Pediatrics 1993;91:969-75.

- $\quad$ 18.Richardson DK, Corcoran JD, Escobar GJ, Lee SK. SNAP-II and SNAPPE-II: simplified newborn illness severity and mortality risks scores. J Pediatr 2001;138:92-100. 
- 19.Gray JE, Richardson DK, McCormick MC, Workman-Daniels K, Goldmann DA. Neonatal Therapeutic Intervention Scoring System: a therapy-based severity-of-illness index. Pediatrics 1992;90:561-7.

- 20.Horbar JD, Onstad L, Wright E, The National Institute of Child Health and Human Development Neonatal Research Network. Predicting mortality risks for infants weighing 501 to 1500 grams at birth: a National Institute of Health Neonatal Research Network report. Crit Care Med 1993; 21:12-8.

- 21.Maier RF, Rey M, Metze BC, Obladen M. Comparison of mortality risk: a score for very low birth weight infants. Arch Dis Child Fetol Neonatol Ed 1997;76:F146-50.

- 22.Garcia H, Villegas-Silva R, Villanueva-Garcia D, et al. Validation of a prognostic index in the critically newborn. Rev Invest Clin 2000;52:406-14

- 23. Ramirez Huerta AC, Glober Paez F, Higareda Almaraz MA. Índice de riesgo clínico para bebés II (CRIB II) y peso para predecir mortalidad en recién nacidos pretérmino (RNP) menores de 32 semanas tratados con surfactante. Gac Med Mex. 2015;151:192-6.

- 24. Mesquita M; Alvarez E, Godoy L, Avalos S.Scores de gravedad SNAP II y SNAP-PE II en la determinación de riesgo de mortalidad neonatal en una unidad de cuidados intensivos polivalente. Pediatr. (Asunción) [online]. 2011, vol.38, n.2, pp. 93-100. ISSN 1683-9803

- 25. Dorling JS, Field DJ, Manktelow B. Neonatal disease severity scoring systems. Arch Dis Child Fetal Neonatal Ed 2005;90:F11-F16. 
- 26.Niramis R, Tangkhabuanbut P, Anuntkosol M, Buranakitjaroen V, Tongsin A, Mahatharadol V. Clinical outcomes of esophageal atresia: comparison between the Waterston and the Spitz classifications. Ann Acad Med Singapore. 2013 Jun;42(6):297-300.

- 27. L. Spitz, E.M. Kiely, J.A. Morecroft, D.P. Drake. .Oesophageal atresia: Atrisk groups for the 1990s. J Pediatr. Surg. , 29, (6), 723-725

- 28. Poenaru D, Laberge JM, Neilson IR, Guttman FM. A new prognostic classification for esophageal atresia. Surgery. 1993 Apr;113(4):426-32.

- 29. Vara Callau M, Royo Pérez D, González Esgueda AJ, Gracia Torralba L, Rodríguez Sanz ML , Clavero Montañés N, el al. Atresia de esófago: estudio descriptivo de una serie de 34 pacientes. Acta Pediatr Esp. 2014; 72(4): 76-80

- 30. Verma RK, Nath SS, Sinha GK. Retrospective study of esophageal atresia with tracheo-esophageal fistula. Three years experience. Indian J. Anaesth. 2003;47 (2):111-115.

- 31. Clark RH, Gordon P, Walker WM, Laughon M, Smith PB, Spitzer AR. Characteristics of patients who die of necrotizing enterocolitis. J Perinatol 2012; 32(3): 199-204.

- 32. Lee JH. An update on necrotizing enterocolitis: pathogenesis and preventive strategies. Korean J Pediatr 2011; 54(9): 368-72.

- $\quad$ 33. Thyoka M, de Coppi P, Eaton S, Khoo K, Hall NJ, Curry J, et al. Advanced necrotizing enterocolitis part 1: mortality. Eur J Pediatr Surg 2012; 22(1): 8-12. 
- 34. Chiu B, Pillai SB, Almond PS, Beth Madonna M, Reynolds M, Luck SR, et al. To drain or not to drain: a single institution experience with neonatal intestinal perforation. J Perinat Med 2006; 34(4): 338-41.

- 35.Lai HS, Lin WH, Chen HA, Fan SZ, Chou HC. Successful laparotomy in a 432g extremelylow-birth-weight infant with focal intestinal perforations. Int Surg. 2010;95(1):57-9.

- 36.Breaux CW Jr, Rouse TM, Cain WS, Georgeson KE. Congenital diaphragmatic hernia in an era of delayed repair after medical and/or extracorporeal membrane oxygenation stabilization: a prognostic and management classification. J Pediatr Surg 1992;27:1192-6.

- 37.Tsao K, Allison ND, Harting MT, Lally PA, Lally KP. Congenital diaphragmatic hernia in the preterm infant. Surgery. 2010;148(2):404-10

- 38.Nixon HH, Tawes R. Etiology and treatment of small intestinal atresia: analysis of a series of 127 jejunoileal atresias and comparison with 62 duodenal atresias. Surgery 1971;69:41-51.

- 39.Burjonrappa S, Crete E, Bouchard S. Comparative outcomes inintestinal atresia: a clinical outcome and pathophysiology analysis. Pediatr Surg Int. 2011;27(4):437-42. 22

- 40.Christison-Lagay ER, Kelleher CM, Langer JC. Neonatal abdominal wall defects. Semin Fetal Neonatal Med. 2011;16(3):164-72. 21.

- $\quad$ 41.Malviya S, Voepel-Lewis T, Chiravuri SD, Gibbons K, Chimbira WT, Nafiu 00, et al. Does an objective system- based approach improve assessment of perioperative risk in children? A preliminary evaluation of the 'NARCO'. Br J Anaesth 2011; 106(3): 352-8.

- $\quad$ 42. Sheikh A. Prognosis of surgical neonates. J Neonat Surg 2012;1:1 
- 43 Manchanda V, Sarin YK, Ramji S. Prognostic factors determining mortality in surgical neonates. J Neonat Surg 2012; 1: 3

- 44. Ugwu RO, Okoro PE. Pattern, outcome and challenges of neonatal surgical cases in a tertiary teaching hospital. Afr J Paediatr Surg. 2013 Jul-Sep;10(3):226-30

- 45. Catré D, Lopes MF, Madrigal A, Oliveiros B, Cabrita AS, Viana JS et al. Predictors of major postoperative complications in neonatal surgery. Rev. Col. Bras. Cir. 2013; 40(5): 363-369

- 46. Catré D, Lopes MF, Madrigal A, Oliveiros B, Viana JS , Cabrita AS. Early mortality after neonatal surgery: analysis of risk factors in an optimized health care system for the surgical newborn. Rev Bras Epidemiol. 2013 Dec;16(4):943-52.

- 47. Contreras M. Evaluación médica preoperatoria como predictor de riesgo quirúrgico y no quirúrgico. Epidemiología en acción. 2010,Vol. 8, No 1, Enero-Julio 83-96.

- 48. Giannoudis PV. Surgical priorities in damage control in polytrauma. Bone Joint Surg [Br] 2003;85-B:478-83.

- 49.Sauaia A, Moore FA, Moore EE, Moser KS, Brennan R, Read RA, Pons PT: Epidemiology of trauma deaths: A reassessment. J Trauma 1995; 38: 185-93

- 50.Mattox K: Introduction, background, and future proyections of damage control surgery. Surg Clin North Am 1997; 77: 753-9

- 51.Moore E: Staged laparotomy for the hypothermia, acidosis, and coagulopathy syndrome. Am J Surg 1996; 172: 405-10 
- 52.Cue JI, Cryer HG, Miller FB, Richardson JD, Polk HC Jr: Packing and planned reexploration for hepatic and retroperitoneal hemorrhage: Critical refinements of a useful technique. J Trauma 1990; 30: 1007-13

- 53. Hirshberg A, Mattox K: Planned reoperation for severe trauma. Ann Surg 1995; 222: 3-8

- 54.Carrillo C, Fogler R, Shaftan G: Delayed gastrointestinal reconstruction following massive abdominal trauma. J Trauma 1993; 34 : $233-5$

- $\quad$ 55.Burch J, Denton J, Noble R: Physiologic rationale for abbreviated laparotomy. Surg ClinNorth Am 1997; 77: 779-82 10.

- 56. Hirshberg A, Walden R: Damage control forabdominal trauma. Surg Clin North Am 1997; 77:813-20 11.

- 57.Morris JA Jr, Eddy VA, Blinman TA,Rutherford EJ, Sharp KW: The staged celiotomy for trauma, issues in unpacking reconstruction. Ann Surg 1993; 217: 576-86

- 58. Tremblay LN, Feliciano DV, Schmidt J, Cava RA, Tchorz KM, et al: Skin only or silo closure in the critically ill patient with an open abdomen. Am J Surg 2001; 182: 670-5

- 59.Broos PL, Janzing HM, Vandermeeren LA, Klockaerts KS. Life saving surgery in polytrauma patients. Przegl Lek. 2000;57 Suppl 5:118-9.

- 60. Perez Navarro AF, Pérez Santos F, Hernández González A, Alonso Marrero C. Valoración diagnóstica y consideraciones terapéuticas del sangrado excesivo en el posoperatorio inmediato de cirugía cardiovascular. Revisión Bibliográfica. Hospital Clínico Quirúrgico "Hermanos Ameijeiras". La Habana, Cuba. 
- 61.Leguisamon, Norman Beatriz. Sangrado como complicación en la cirugía cardiaca. Revista Latinoamericana de la CEC. 2006;XIII(1)

- 62. Roque Pifarré. Management of bleeding in cardiovascular surgery. Filadelfia: Hanley \& Belfus, inc. 2000. p. 307-322.

- 63. Chuquiure-Valenzuela E . Evaluación del riesgo hemorrágico en cirugía cardíaca. Anestesia Cardiovascular. 2008;31 Supl.1:S121-S126.

- $\quad$ 64. Carvajal R, Londoño A. Factores de riesgo e infección del sitio quirúrgico en procedimientos de cirugía ortopédica con prótesis. Rev Chilena Infectol 2012; 29 (4): 395-400.

- 65. Despaigne Alba I, Rodríguez Fernández Z, Romero García LI, Pascual Bestard M, Ricardo Ramírez JM. Morbilidad y mortalidad por infecciones posoperatorias. Rev Cubana Cir [online]. 2013, vol.52, n.1, pp. 13-24. ISSN 0034-7493.

- 66. E. Nve Obiang, J.M. Badia. Infección de sitio quirúrgico: definición, clasificación y factores de riesgo. Guía Clínica de Infecciones Quirúrgicas. Capítulo 5. 95-116.

- 67.Barone JE, Tucker JB, Cecere J, Ion MY, Reinhard E, Blabey RG Jr, et al. Hypothermia does not result in more complications after colon surgery. Am Surg 1999; 65: 356-9.

- 68.Cainzos M. Surgical infection control. Surgical Infection SocietyEurope Presidential Address. Surg Infec 2005; 6: 7-17.

- 69.CDC NNIS System. National Nosocomial Infections Surveillance (NNIS) System report, data summary from January 1992 through June 2004, issued October 2004. Am J Infect Control 2004; 32: 470-85. 
- 70.Culver DH, Horan TC, Gaynes RP, Martone WJ, Jarvis WR, Emori TG, et al, and the National Nosocomial Infections Surveillance System. Surgical wound infection rates by wound class, operative procedure, and patient risk index. Am J Med 1991; 91: 152S-7S.

- 71.Garibaldi RA, Cushing D, Lerer T. Risk factors for postoperative infection. Am J Med 1991; 91 (Supl. 3B): $158 S$.

- 72. Bruno M. Hospital CG Durand. Recomendaciones para la prevención de infecciones en el sitio quirúrgico. Fundación Neonatológica Miguel Larguía. Argentina

- 73. Parente A, Cañizo A, Huerga A, Laín A, Fanjul M, . Carrera N et al. ¿Es adecuado utilizar las Unidades de Cuidados Intensivos Neonatales como sala de operaciones?.CirPediatr2009;22:61-64.

- 74.Zinder CL. Outcome análisis for gastroschisis. J Pediatr Surg 1999; 34: $1253-1256$.

- 75. Lee SK, McMillan DD, Ohlsson A, et al. Variations in practise and outcomes in the Canadian NICU Network: 1996-1997. Pediatrics 2000; 106:1070-1079.

- 76.Skarsgard ED, Blair GK, Lee SK. Toward evidence-based practi- ces in neonatal surgical care-I: The Canadian NICU network. J Pediatr Surg 2003; 38: 672-677.

- 77.Gould DS, Montenegro LM, Gaynor JW, Lacy SP, Ittenbach R, et al. A comparison of on-site and off-site patent ductus arteriosus ligation in premature infants. Pediatrics 2003; 112:1298-1301. 
- 78.Frawley G, Bayley G, Chondros P. Laparotomy for necrotizing enterocolitis: intensive care nursery compared with operating theatre. $J$ Paediatr Child Health 1999; 35: 291-295

- 79. Finner NN, Woo BC, Hayashi A, Hayes B. Neonatal surgery: intensive care unit versus operating room. J Pediatr Surg 1993; 28:645- 649

- 80.Strauss RG. Transfusion therapy in neonates. Am J Dis Child. 1991;145:904-11. 2. • Roseff SD, Luban NLC, Manno CS. Guidelines for assessing appropriate-ness of pediatric transfusion. Transfusion. 2002;42:1398-413.

- 81. Wong ECC, Luban NLC. Intrauterine, neonatal and pediatric transfusion. En: Mintz PD, editor. Transfusion Therapy. Clinical Principles and Practice. 2nd ed. Bethesda: AABB Press; 2005. p. 15992.

- 82.British Committee for Standards in Haematology. Transfusion guidelines for neonates and older children. $\mathrm{Br} \mathrm{J}$ Haematol. 2004;124:433-53.

- 83. Amendments and corrections to the Transfusion guidelines for neonates and older children (BCSH, 2004a) and to the Guidelines for the use of fresh frozen plasma, cryoprecipitate and cryosupernatant $(\mathrm{BSCH}$, 2004b). Br J Haematol. 2006;136:514-6.

- 84. Barbolla L, Contreras E. Indicación de la transfusión en situaciones especiales: trasplantes, cuidados intensivos, cardiopatías, neonatología y pediatría. En: Barbo- Ila L, Contreras E, Pujol MM, editores. Manual práctico de medicina transfusio- nal. FEHH. Madrid: 2002. p. 105-23 
- 85.Mannuccio Mannucci P. Hemostatic drugs. N Engl J Med 1998,: 339 : 245-253.

- 86.Metz S, Horrow JC. Update on aprotinin and drugs to promote coagulation. Advances in Anesthesia 1996; 13: 355-392.

- 87.Stack G and Snyder EL. Alternatives to perioperative blood transfusion. Advances in Anesthesia 1991; 8: 209-239.

- 88.Fremes SE, Wong BI, Lee E, Mai R, Christakis G, McLean RF et al. Metaanalysis of prophylactic drug treatment in the prevention of postoperative bleeding. Ann Thorac Surg 1994; 58: 1580- 1588.

- 89.Mertes N, Booke M and Aken V. Strategies to reduce the need for perioperative blood transfusion. European Academy of Anaesthesiology 1997; 14: 24-34.

- 90.Bulligham A, Priestley M. Anesthetic manegement of patients being treated with anticoagulants. Current opinion in anaesthesiology 1997; 10:234-239.

- 91.Porte Rj, Leebeek FWK. Pharmacological strategies to decrease transfuion requirements in patients undergoing surgery. Drugs 2002; 62: 2193-2211.

- 92.Koh MBC, Hunt BJ. The management of perioperative bleeding. Blood reviewers 2003; 17:179- 185.

- 93. Franchini M, Zaffanello M and Veneri D. Recombinant Factor VIla an update on its clinical use.

- 94.Thromb Haemost 2005; 93: 1027-35. 20. Roberts HR, Monroe D and Escobar MA. Current concepts of hemostasis implications for therapy. Anesthesiology 2004; 100: 722-30 
- 95.Zupancic J, Richardson D, O'Brien B, Schmidt B, MC W. Daily cost prediction model in neonatal intensive care. Int J Tech Ass Health Care. 2003;19:330-8.

- 96.Neonatal intensive care for low birth weight infants: Costs and effectiveness (Health Technology Case Study 38). Washington D.C: Office of Technology Assessment1987.

- 97.Narang A, Kiran PS, P K. Cost of Neonatal Intensive Care in a Tertiary Care Cente. Indian Pediatrics. 2005;42: 989-97.

- 98. Shanmugasundaram R, Padmapriya E, Shyamala J. Cost of neonatal intensive care. Indian J Pediatr. 1998;65: 249-55. 
TABLA XI 


\begin{tabular}{|c|c|c|c|c|c|c|c|c|c|}
\hline $\begin{array}{l}\dot{0} \\
\end{array}$ & 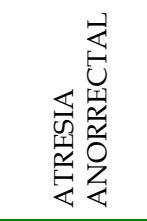 & 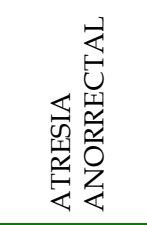 & 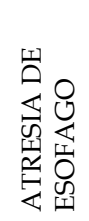 & 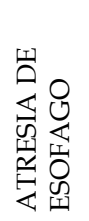 & 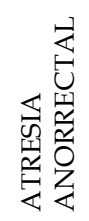 & 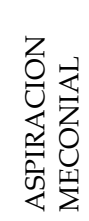 & 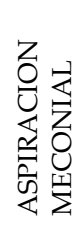 & 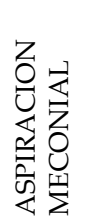 & 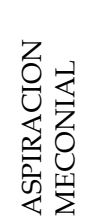 \\
\hline $\begin{array}{l}0 \\
0 \\
\frac{\alpha}{\alpha}\end{array}$ & 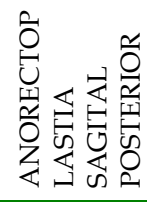 & 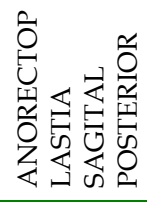 & 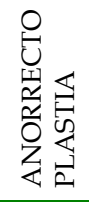 & 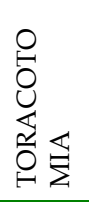 & 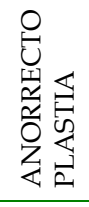 & $\sum_{i=1}^{0}$ & $\sum_{i}^{0}$ & $\sum_{i=1}^{0}$ & $\sum_{i=1}^{0}$ \\
\hline z & పี & 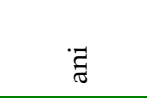 & 妾 & 妾 & 文 & z & ż & ż & $\begin{array}{l}z \\
\mathrm{~J} \\
\mathrm{D}\end{array}$ \\
\hline$\frac{\text { 玄 }}{\underline{\underline{u}}}$ & ‡ & के & $\vec{\omega}$ & $\bar{\omega}$ & そ & そ) & ๘ & $\bar{\omega}$ & क \\
\hline$\sum_{0}^{\circ}$ & ‡ & 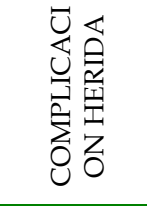 & 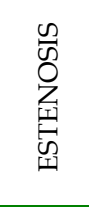 & 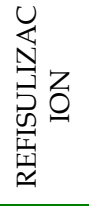 & $\stackrel{̊}{Z}$ & $\stackrel{O}{Z}$ & $\stackrel{O}{Z}$ & そ) & そ) \\
\hline 몽 & 怘 & 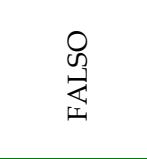 & 另 & 䧄 & 怘 & 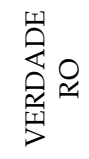 & 怘 & 咽 & 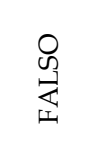 \\
\hline ִִ & $\underset{-}{\stackrel{8}{-}}$ & $\underset{-}{\stackrel{8}{-}}$ & $\therefore \stackrel{0}{0}$ & 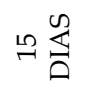 & $\underset{\sqsupset}{\leftrightarrows}$ & 芒 & $=\underset{0}{\mathbb{2}}$ & $\stackrel{4}{\sim}$ & तु \\
\hline$\underset{4}{4}$ & $\frac{\overbrace{0}^{\infty} 8}{0}$ & $\begin{array}{l}\frac{0}{2} 8 \\
\frac{8}{2} \\
\frac{1}{0}\end{array}$ & 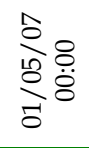 & 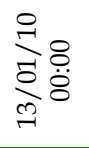 & 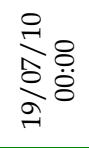 & 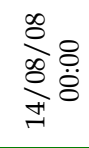 & $\underset{\substack{\infty \\
\Rightarrow}}{\infty} \stackrel{0}{0}$ & 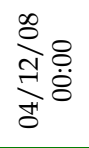 & 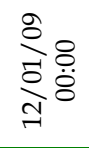 \\
\hline$\dot{\vec{u}}$ & 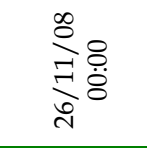 & 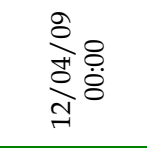 & 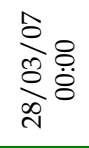 & 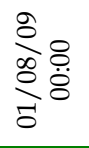 & 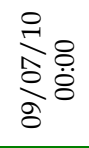 & 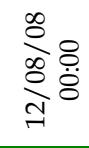 & $\begin{array}{l}\stackrel{\infty}{0}_{0}^{\infty} \\
\stackrel{0}{0} \\
0\end{array}$ & 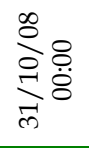 & 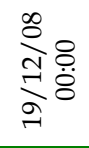 \\
\hline$z_{L}$ & 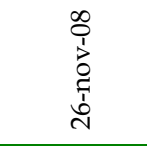 & 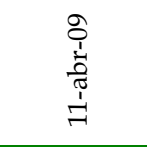 & 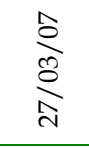 & 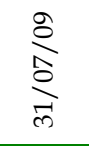 & $\underset{\infty}{\stackrel{9}{\infty}}$ & $\underset{\substack{\infty \\
\stackrel{\infty}{\infty}}}{\infty}$ & $\underset{\frac{1}{8}}{\stackrel{\infty}{8}}$ & $\underset{\infty}{\stackrel{\infty}{\rho}}$ & 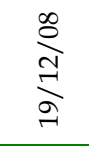 \\
\hline $\begin{array}{l}\mathscr{C} \\
\text { I }\end{array}$ & 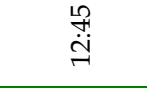 & त्ञ & 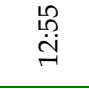 & 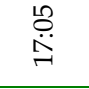 & 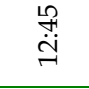 & 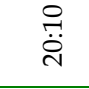 & $\begin{array}{l}\text { @̊ } \\
\stackrel{0}{0}\end{array}$ & $\begin{array}{l}8 \\
\stackrel{8}{\text { 巾े }}\end{array}$ & 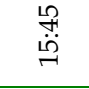 \\
\hline 높 & 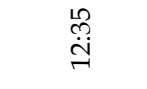 & 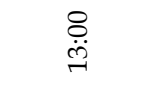 & 矛 & 员 & 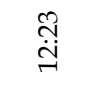 & $\begin{array}{l}\text { ஜ̊ } \\
\stackrel{\leftrightarrow}{\circ}\end{array}$ & $\stackrel{\stackrel{\leftrightarrow}{0}}{\stackrel{\leftrightarrow}{*}}$ & 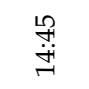 & 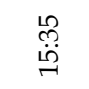 \\
\hline $\bar{x}$ & $\begin{array}{l}\stackrel{\text { Pf }}{\stackrel{9}{0}}\end{array}$ & 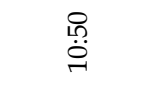 & $\begin{array}{l}\stackrel{9}{0} \\
\stackrel{0}{0}\end{array}$ & 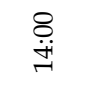 & 号 & $\begin{array}{l}\stackrel{\infty}{0} \\
\stackrel{\sigma}{\sigma}\end{array}$ & 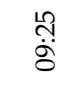 & $\begin{array}{l}\stackrel{0}{0} \\
\stackrel{\oplus}{\oplus}\end{array}$ & 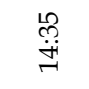 \\
\hline ب & 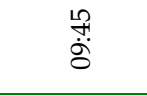 & $\begin{array}{l}\text { ஷ̊. } \\
\text { g̊ }\end{array}$ & $\stackrel{\stackrel{n}{\circ}}{\ddot{\sigma}}$ & $\stackrel{\stackrel{\leftrightarrow}{\stackrel{n}{ت}}}{=}$ & $\begin{array}{l}\stackrel{R}{\leftrightarrow} \\
\dot{\sigma}\end{array}$ & 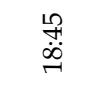 & $\begin{array}{l}0 \\
\ddot{0} \\
\ddot{0}\end{array}$ & $\begin{array}{l}\stackrel{0}{\ddot{m}} \\
\stackrel{\vec{n}}{ }\end{array}$ & 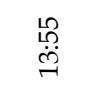 \\
\hline 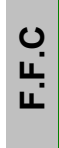 & $\begin{array}{c}\infty \\
0 \\
1 \\
0 \\
\vdots \\
1 \\
\text { 彳े }\end{array}$ & 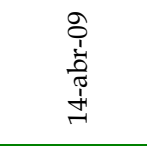 & 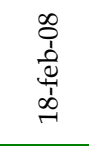 & 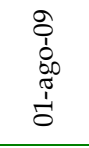 & $\begin{array}{l}0 \\
\text { 1 } \\
\frac{1}{3} \\
\frac{1}{2} \\
\overrightarrow{7}\end{array}$ & 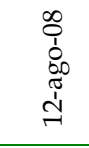 & 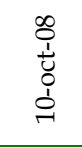 & $\begin{array}{l}\infty \\
0 \\
1 \\
0 \\
\vdots \\
0 \\
0\end{array}$ & 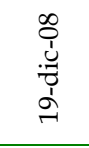 \\
\hline$\frac{0}{4}$ & 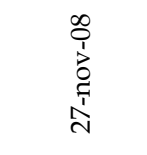 & 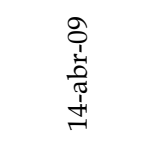 & 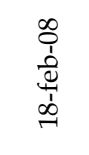 & 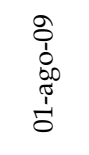 & $\begin{array}{l}0 \\
\frac{1}{3} \\
\frac{7}{1} \\
\frac{1}{1}\end{array}$ & 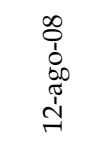 & 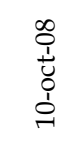 & $\begin{array}{l}\infty \\
0 \\
\vdots \\
0 \\
\vdots \\
\vdots \\
0\end{array}$ & 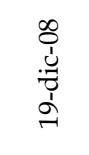 \\
\hline$\stackrel{\pi}{\mathbf{z}}$ & 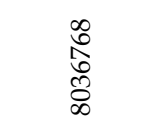 & 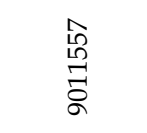 & 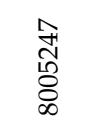 & 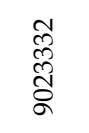 & 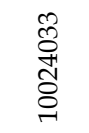 & $\begin{array}{l}\text { त̃ } \\
\text { నิ } \\
\text { రీ }\end{array}$ & $\begin{array}{l}0 \\
\text { 今े } \\
\text { ర్ } \\
\infty\end{array}$ & 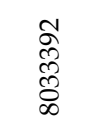 & $\begin{array}{l}0 \\
\infty \\
\infty \\
\infty \\
0 \\
\infty \\
\infty\end{array}$ \\
\hline
\end{tabular}




\begin{tabular}{|c|c|c|c|c|c|c|c|c|}
\hline 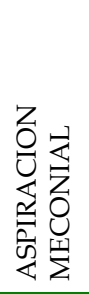 & 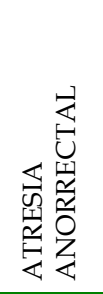 & 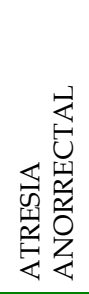 & 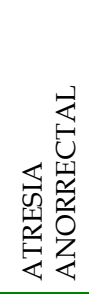 & 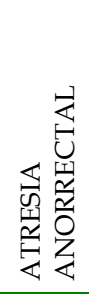 & 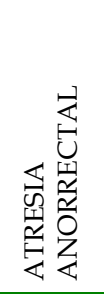 & 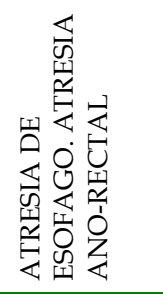 & 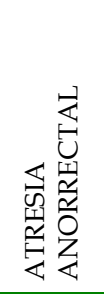 & 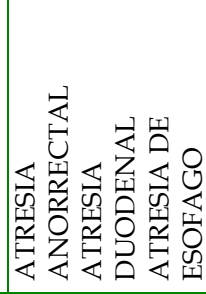 \\
\hline$\sum_{i}^{0}$ & $\begin{array}{l}0 \\
0 \\
0 \\
\frac{\pi}{4} \\
\vdots \\
\vdots\end{array}$ & 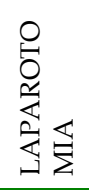 & 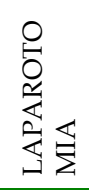 & 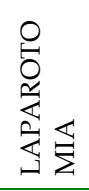 & 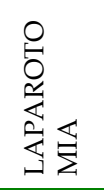 & 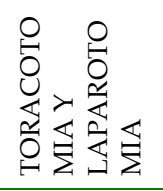 & 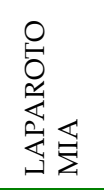 & 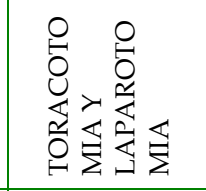 \\
\hline $\begin{array}{l}z \\
y \\
0\end{array}$ & 文 & 文 & 芩 & $\bar{z}$ & 文 & $\bar{z}$ & 云 & 云 \\
\hline $\bar{\omega}$ & 尺 & 尺 & क & 尺 & 尺 & ๘ & $\bar{\omega}$ & 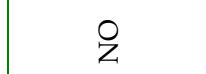 \\
\hline ○ & ○ & $\stackrel{\circ}{Z}$ & 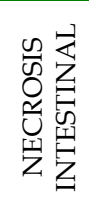 & ○ & 尺े & 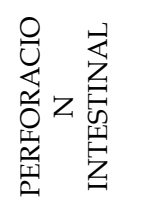 & 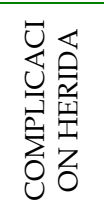 & $\stackrel{\circ}{Z}$ \\
\hline 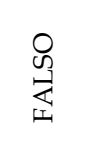 & 怘 & 怘 & 忌 & 㣽 & 㣽 & 只 & 邑 & 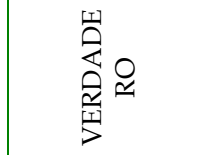 \\
\hline$\stackrel{0}{\sim}$ & $\underset{\sim}{\overleftrightarrow{\Delta}}$ & $\underset{\text { 足 }}{\stackrel{N}{\Delta}}$ & 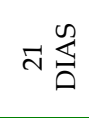 & 足 & $\underset{\neg}{\overleftrightarrow{\Xi}}$ & 임 & 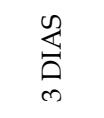 & ণ্ড \\
\hline 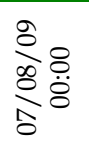 & 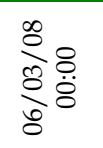 & 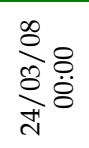 & 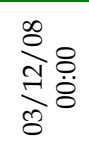 & $\begin{array}{l}\frac{a}{2} \\
\text { ते } \\
\frac{\delta}{0}\end{array}$ & 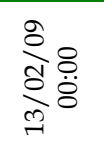 & 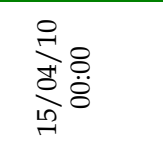 & 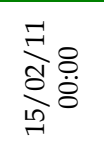 & 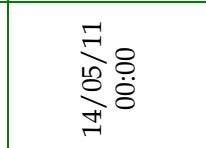 \\
\hline 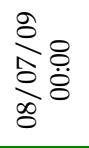 & $\frac{\stackrel{\infty}{0}_{0}^{\infty} 8}{0}$ & 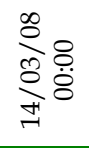 & 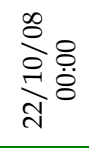 & 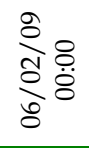 & 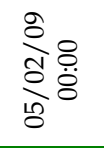 & 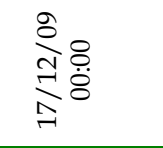 & 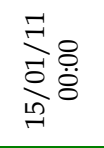 & 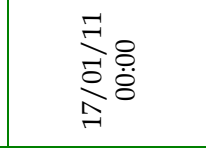 \\
\hline 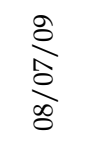 & $\frac{\stackrel{\infty}{\infty}}{\frac{\infty}{\delta}}$ & $\frac{\infty}{\stackrel{\infty}{\infty}}$ & 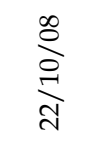 & $\frac{\stackrel{\rho}{\partial}}{\frac{\rho}{\infty}}$ & 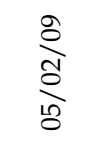 & 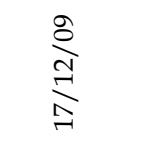 & 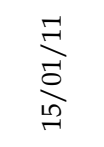 & $\underset{ }{\stackrel{ }{ }}$ \\
\hline $\begin{array}{l}\stackrel{\circ}{\leftrightarrow} \\
\stackrel{\leftrightarrow}{\sigma}\end{array}$ & 号 & 㤐 & $\begin{array}{c}\stackrel{\leftrightarrow}{\dddot{U}} \\
\stackrel{u}{U}\end{array}$ & 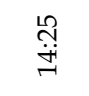 & $\underset{\stackrel{\leftrightarrow}{0}}{\stackrel{\leftrightarrow}{\leftrightarrow}}$ & 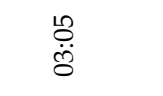 & 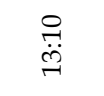 & 号 \\
\hline 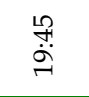 & 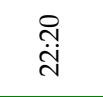 & $\begin{array}{l}\stackrel{\leftrightarrow}{\infty} \\
\stackrel{\leftrightarrow}{+}\end{array}$ & 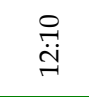 & $\begin{array}{l}\stackrel{8}{+} \\
\stackrel{+}{+}\end{array}$ & $\begin{array}{l}\stackrel{9}{4} \\
\text { ì }\end{array}$ & $\begin{array}{l}\stackrel{\leftrightarrow}{\mathscr{U}} \\
\stackrel{\delta}{\delta}\end{array}$ & 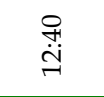 & $\underset{\stackrel{P}{+}}{\stackrel{f}{\sim}}$ \\
\hline 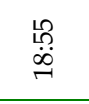 & 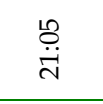 & $\begin{array}{l}\stackrel{\oplus}{\pi} \\
\ddot{\sim}\end{array}$ & $\begin{array}{l}\stackrel{\leftrightarrow}{0} \\
\stackrel{\leftrightarrow}{\oplus}\end{array}$ & $\stackrel{\stackrel{\leftrightarrow}{ت}}{ت}$ & 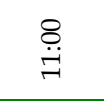 & $\begin{array}{l}8 \\
\ddot{\sim} \\
\text { ते }\end{array}$ & $\begin{array}{l}\stackrel{9}{H} \\
\ddot{ت}\end{array}$ & 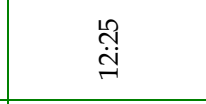 \\
\hline $\begin{array}{l}\stackrel{\mathscr{L}}{\ddot{\infty}} \\
\stackrel{\dot{\theta}}{\sim}\end{array}$ & ஜ்̊̀ & 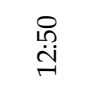 & $\begin{array}{l}\stackrel{\leftrightarrow}{\Lambda} \\
\ddot{g}\end{array}$ & 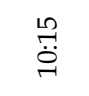 & $\stackrel{8}{\stackrel{0}{\oplus}}$ & $\begin{array}{l}\stackrel{\leftrightarrow}{m} \\
\stackrel{\vec{n}}{n}\end{array}$ & 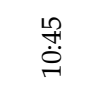 & $\stackrel{\stackrel{\text { Pf }}{ت}}{ت}$ \\
\hline $\begin{array}{l}0 \\
0 \\
\frac{1}{3} \\
\frac{7}{1} \\
0 \\
0\end{array}$ & 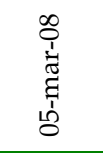 & 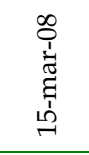 & 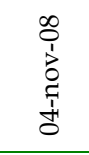 & 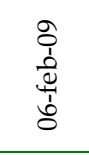 & $\begin{array}{l}\text { oे } \\
\text { क्षे } \\
\text { bे }\end{array}$ & 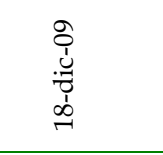 & $\begin{array}{l}\vec{D} \\
\dot{d} \\
\dot{0} \\
\stackrel{0}{0}\end{array}$ & 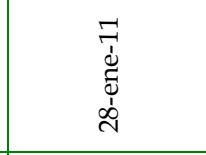 \\
\hline $\begin{array}{l}0 \\
0 \\
\frac{1}{.7} \\
\frac{7}{1} \\
0 \\
0\end{array}$ & 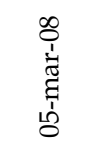 & 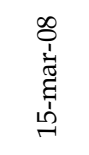 & 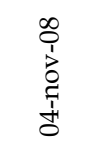 & $\begin{array}{l}8 \\
0 \\
1 \\
\frac{1}{2} \\
\text { है }\end{array}$ & 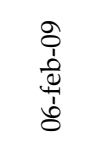 & 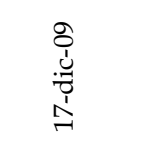 & $\begin{array}{l}\overrightarrow{1} \\
\dot{d} \\
\dot{0} \\
\stackrel{1}{0}\end{array}$ & $\begin{array}{l}\vec{d} \\
\stackrel{d}{0} \\
0 \\
\text { d }\end{array}$ \\
\hline 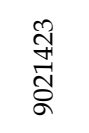 & 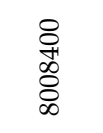 & 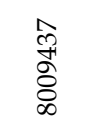 & $\begin{array}{l}\text { o } \\
\infty \\
\infty \\
\infty \\
\infty\end{array}$ & 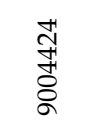 & 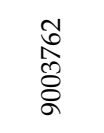 & 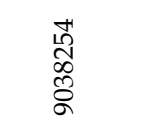 & 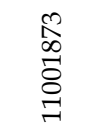 & 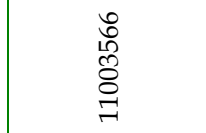 \\
\hline
\end{tabular}




\begin{tabular}{|c|c|c|c|c|c|c|c|c|c|}
\hline 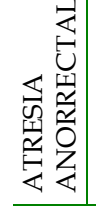 & 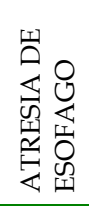 & 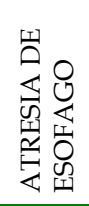 & 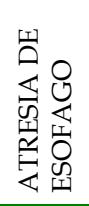 & 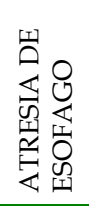 & 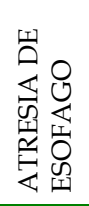 & 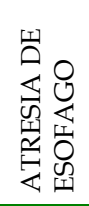 & 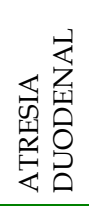 & 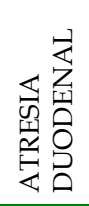 & 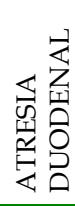 \\
\hline 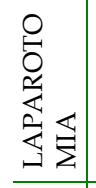 & 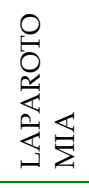 & 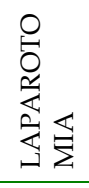 & 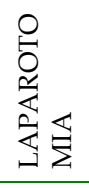 & 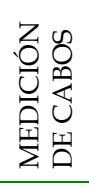 & 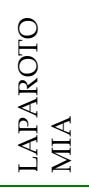 & 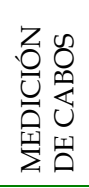 & 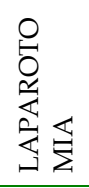 & 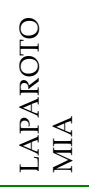 & 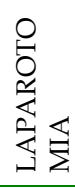 \\
\hline 文 & 文 & 妾 & 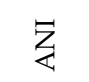 & 文 & 文 & 妾 & 云 & 云 & 文 \\
\hline そ & $\stackrel{\bigcirc}{Z}$ & $\bar{\omega}$ & $\ddot{\omega}$ & $\bar{\omega}$ & क & $\bar{\omega}$ & $\stackrel{\circ}{z}$ & そ) & 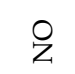 \\
\hline そ) & そ & 尺 & そ & ○ & $\stackrel{\circ}{Z}$ & そ) & $\stackrel{\circ}{Z}$ & $\stackrel{\circ}{Z}$ & 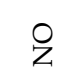 \\
\hline 足 & 怘 & 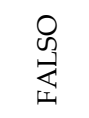 & 惫 & 怘 & 怘 & $\begin{array}{l}0 \\
\text { 足 } \\
\text { 至 }\end{array}$ & 足 & 足 & 怘 \\
\hline$\underset{ન}{\overleftrightarrow{\Delta}}$ & $\underset{\sim}{\overleftrightarrow{\Delta}}$ & 品 & オ这 & 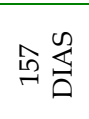 & 点量 & ৯ & $\underset{\text { D }}{\stackrel{\infty}{\Delta}}$ & 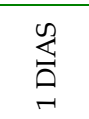 & $\sum_{\infty}^{\infty}$ \\
\hline 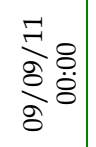 & 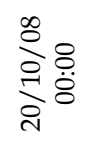 & 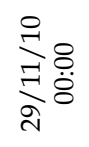 & 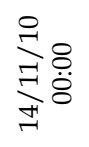 & 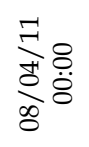 & 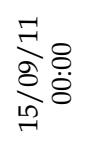 & 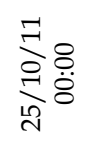 & 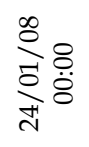 & 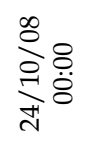 & 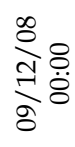 \\
\hline 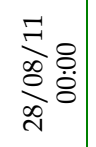 & 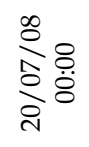 & 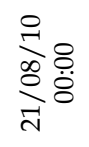 & 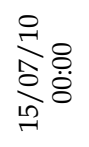 & 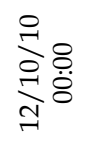 & 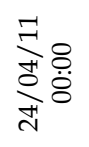 & 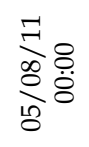 & $\begin{array}{l}\stackrel{D}{0}_{0}^{\infty} \stackrel{0}{0} \\
\overbrace{0}^{\circ}\end{array}$ & $\stackrel{\infty}{0}_{0}^{\infty} 8$ & 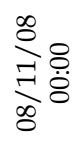 \\
\hline 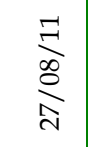 & $\underset{\substack{\infty \\
\stackrel{0}{0}}}{\stackrel{\infty}{0}}$ & $\frac{\stackrel{0}{7}}{\stackrel{\infty}{\ominus}}$ & $\underset{\stackrel{2}{2}}{\stackrel{0}{2}}$ & 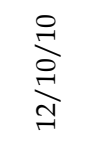 & $\underset{\vec{d}}{\stackrel{\vec{d}}{\vec{d}}}$ & $\frac{\overrightarrow{7}}{\stackrel{0}{2}}$ & $\frac{\infty}{\stackrel{\infty}{\rho}}$ & 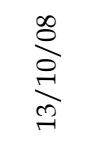 & $\underset{\infty}{\stackrel{\infty}{\ominus}}$ \\
\hline 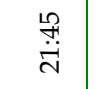 & 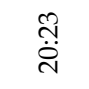 & 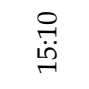 & 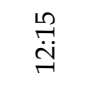 & 品 & 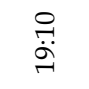 & 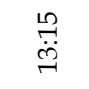 & $\begin{array}{l}\stackrel{\leftrightarrow}{.} \\
\ddot{\ddot{v}}\end{array}$ & $\begin{array}{l}\stackrel{0}{\leftrightarrow n} \\
\ddot{\tilde{n}}\end{array}$ & 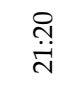 \\
\hline $\begin{array}{l}\stackrel{\leftrightarrow}{\stackrel{n}{n}} \\
\stackrel{n}{n}\end{array}$ & 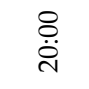 & 吊 & $\underset{\stackrel{\leftrightarrow}{\sim}}{\stackrel{8}{u}}$ & 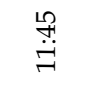 & $\begin{array}{l}\stackrel{0}{0} \\
\stackrel{0}{\sim} \\
\rightarrow\end{array}$ & $\begin{array}{l}\stackrel{\leftrightarrow}{.} \\
\stackrel{\leftrightarrow}{\longrightarrow}\end{array}$ & $\begin{array}{c}\stackrel{0}{\sharp} \\
\ddot{\sim}\end{array}$ & $\begin{array}{l}\infty \\
\dddot{\sim} \\
\ddot{n}\end{array}$ & 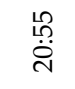 \\
\hline 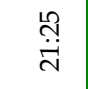 & 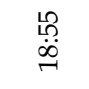 & $\begin{array}{l}\stackrel{2}{\dddot{H}} \\
\stackrel{\oplus}{n}\end{array}$ & 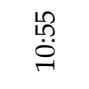 & 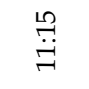 & 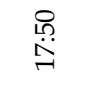 & $\begin{array}{l}\stackrel{\oplus}{\dddot{U}} \\
\text { }\end{array}$ & $\begin{array}{l}\text { तิ } \\
\text { ते }\end{array}$ & $\begin{array}{l}\stackrel{P}{\ddot{⿰}} \\
\text { ते }\end{array}$ & $\begin{array}{l}\text { ̣̊̊ } \\
\text { ते }\end{array}$ \\
\hline 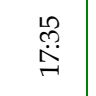 & 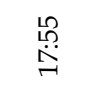 & 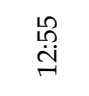 & $\begin{array}{l}\stackrel{\oplus}{0} \\
\stackrel{\leftrightarrow}{\leftrightarrow}\end{array}$ & 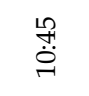 & $\stackrel{\infty}{\ddot{\ddot{\theta}}}$ & 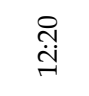 & $\begin{array}{l}\stackrel{8}{0} \\
\dot{\circ}\end{array}$ & $\begin{array}{l}\stackrel{8}{.} \\
\text { ت̇ }\end{array}$ & $\begin{array}{l}\text { ণิ } \\
\stackrel{\sim}{\sim}\end{array}$ \\
\hline 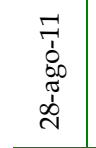 & $\begin{array}{l}\infty \\
0 \\
\frac{1}{3} \\
\frac{3}{1} \\
\text { in }\end{array}$ & 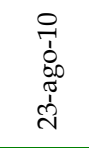 & 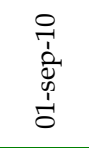 & 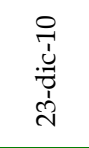 & 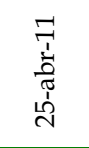 & 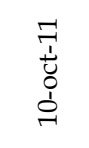 & 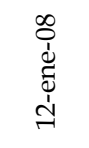 & 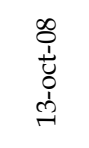 & $\begin{array}{l}\infty \\
0 \\
\grave{1} \\
0 \\
\vdots \\
0\end{array}$ \\
\hline 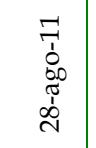 & $\begin{array}{l}\infty \\
0 \\
\frac{1}{2} \\
\frac{2}{1} \\
\text { d. }\end{array}$ & 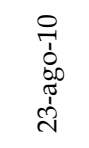 & 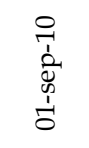 & 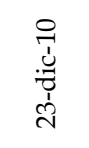 & 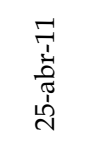 & $\begin{array}{l}\overrightarrow{7} \\
\stackrel{\overrightarrow{1}}{0} \\
0 \\
0\end{array}$ & 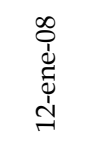 & 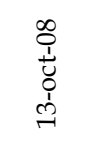 & $\begin{array}{l}\infty \\
0 \\
1 \\
0 \\
0 \\
0 \\
0\end{array}$ \\
\hline 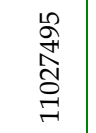 & 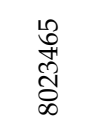 & 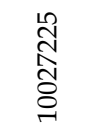 & 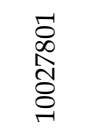 & 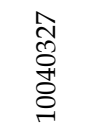 & 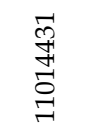 & 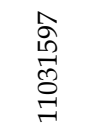 & 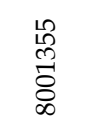 & $\begin{array}{l}\text { No } \\
\hat{0} \\
\stackrel{0}{\infty} \\
\infty\end{array}$ & 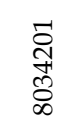 \\
\hline
\end{tabular}




\begin{tabular}{|c|c|c|c|c|c|c|c|c|c|c|}
\hline 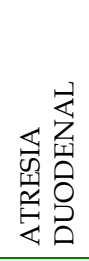 & 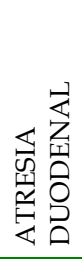 & 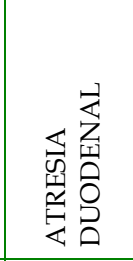 & 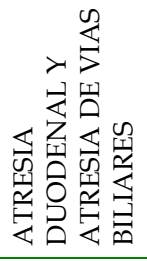 & 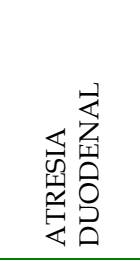 & 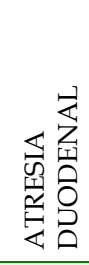 & 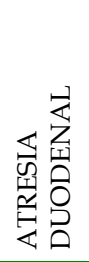 & 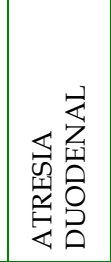 & 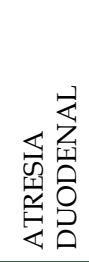 & 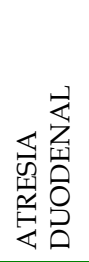 & 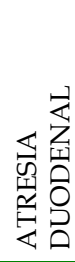 \\
\hline 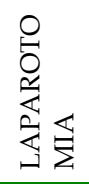 & 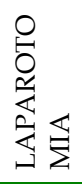 & 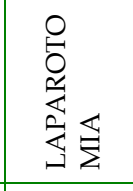 & 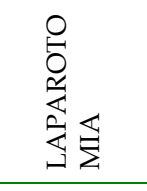 & 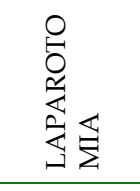 & 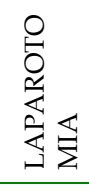 & 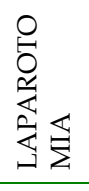 & 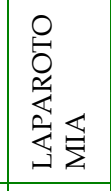 & 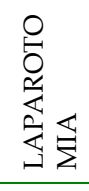 & 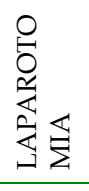 & 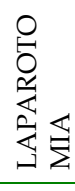 \\
\hline $\bar{z}$ & 文 & $\bar{z}$ & 文 & 芩 & 云 & 文 & 文 & 㓂 & 云 & 云 \\
\hline そ) & Z & $\varpi$ & $\stackrel{\circ}{Z}$ & $\bar{\omega}$ & そ) & そ) & $\bar{\omega}$ & そ) & そ) & そ \\
\hline$\stackrel{\bigcirc}{Z}$ & そ & 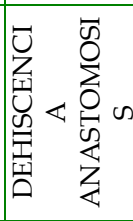 & そ) & 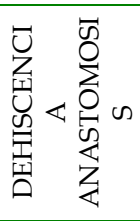 & そ) & 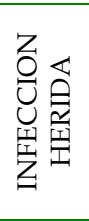 & 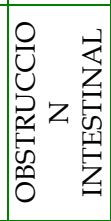 & $\stackrel{\circ}{z}$ & そ) & そ) \\
\hline 唃 & 蒫 & 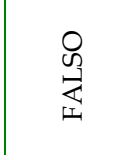 & 怘 & 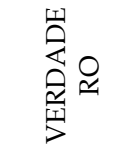 & 思 & 朶 & 怘 & 务 & 忌 & 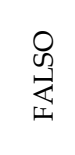 \\
\hline$\underset{\neg}{\overleftrightarrow{D}}$ & $\begin{array}{l}\text { 足 } \\
\text { 竞 }\end{array}$ & হ是 & 离 & 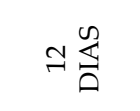 & 胥 & 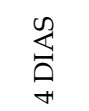 & న结 & 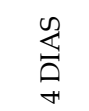 & 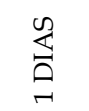 & 离 \\
\hline 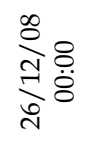 & $\frac{0}{2} 8$ & 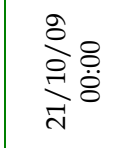 & 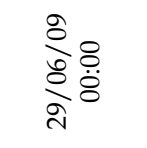 & 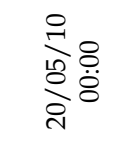 & 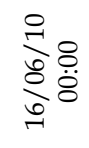 & 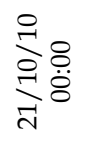 & 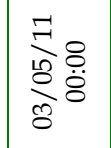 & $\begin{array}{l}\vec{F} 8 \\
\text { ¿े } \\
\text { के }\end{array}$ & 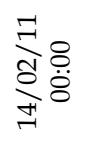 & 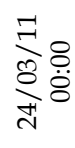 \\
\hline 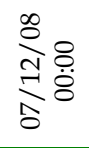 & 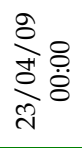 & 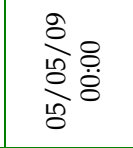 & 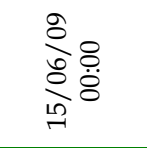 & 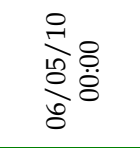 & 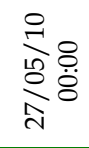 & $\underset{\text { 싱 }}{\sum_{0}^{2}} \stackrel{0}{0}$ & 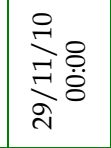 & 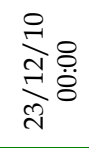 & 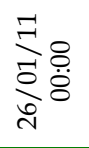 & 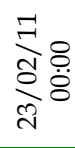 \\
\hline$\frac{\substack{0 \\
\frac{1}{\delta}}}{\Delta}$ & $\underset{\text { dे }}{\stackrel{2}{े}}$ & $\frac{\substack{2 \\
\frac{10}{2}}}{10}$ & $\frac{8}{8}$ & $\frac{0}{\frac{0}{20}}$ & 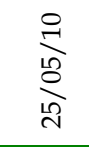 & $\underset{\text { 잉 }}{\stackrel{0}{8}}$ & $\underset{\text { নे }}{\stackrel{8}{7}}$ & 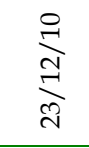 & 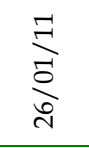 & 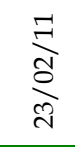 \\
\hline 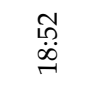 & $\stackrel{\stackrel{\leftrightarrow}{\pi}}{\ddot{n}}$ & 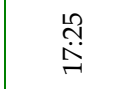 & 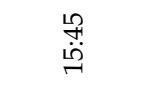 & 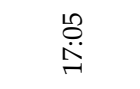 & 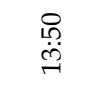 & 号 & $\begin{array}{l}\stackrel{\text { P }}{\sharp} \\
\underset{\sim}{*}\end{array}$ & 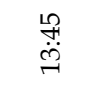 & 员 & 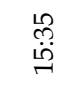 \\
\hline $\begin{array}{l}\stackrel{\Upsilon ્}{\infty} \\
\stackrel{\oplus}{\sim}\end{array}$ & 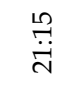 & 華 & $\begin{array}{l}\text { مे } \\
\text { ị. }\end{array}$ & 葛 & $\begin{array}{l}\stackrel{0}{m} \\
\ddot{n}\end{array}$ & 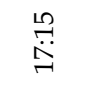 & 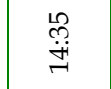 & $\begin{array}{l}\stackrel{0}{m} \\
\text { ले }\end{array}$ & 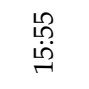 & 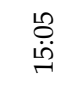 \\
\hline 足 & 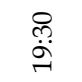 & $\begin{array}{l}\stackrel{2}{\ddot{ت}} \\
\ddot{ت}\end{array}$ & 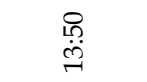 & 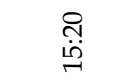 & 号 & $\stackrel{\stackrel{\llcorner}{\ddot{*}}}{\underset{\sim}{*}}$ & 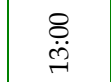 & 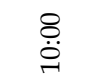 & $\underset{\stackrel{\leftrightarrow}{+}}{\stackrel{+}{*}}$ & 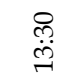 \\
\hline 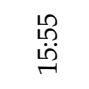 & 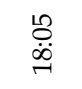 & 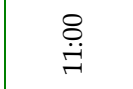 & $\stackrel{\stackrel{0}{\ddot{I}}}{ }$ & 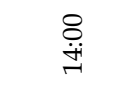 & 号 & $\begin{array}{l}\stackrel{9}{\ddot{H}} \\
\stackrel{7}{*}\end{array}$ & 总 & $\begin{array}{l}\stackrel{8}{0} \\
\dot{0}\end{array}$ & 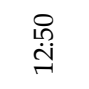 & 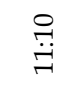 \\
\hline 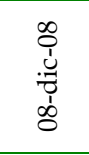 & 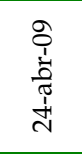 & 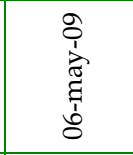 & 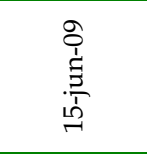 & 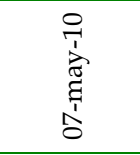 & 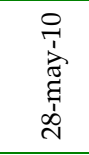 & $\begin{array}{l}0 \\
\stackrel{1}{1} \\
0 \\
\dot{0} \\
\dot{0}\end{array}$ & 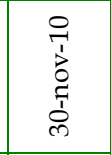 & 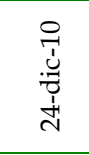 & 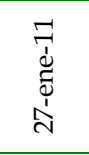 & 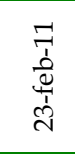 \\
\hline 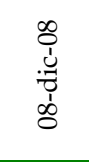 & 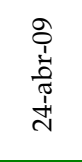 & 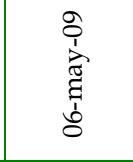 & 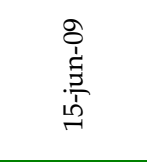 & 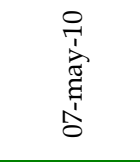 & 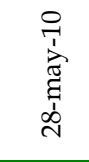 & $\begin{array}{l}0 \\
\stackrel{1}{1} \\
\dot{0} \\
\dot{0} \\
0\end{array}$ & 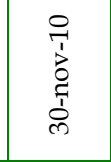 & 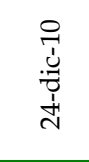 & 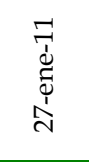 & 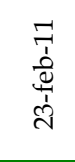 \\
\hline 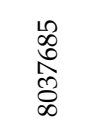 & 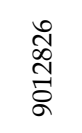 & 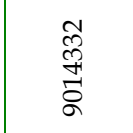 & $\begin{array}{l}\circ \\
\infty \\
\infty \\
\infty \\
5 \\
\vdots\end{array}$ & 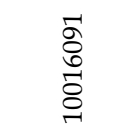 & 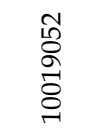 & 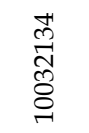 & 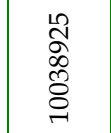 & 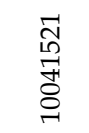 & 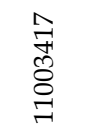 & 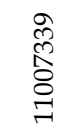 \\
\hline
\end{tabular}




\begin{tabular}{|c|c|c|c|c|c|c|c|c|c|}
\hline 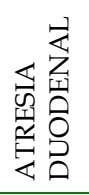 & 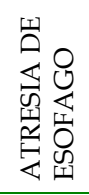 & 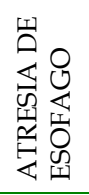 & 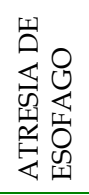 & 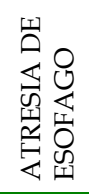 & 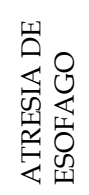 & 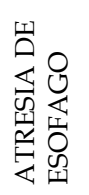 & 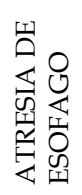 & 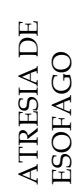 & 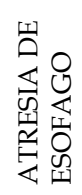 \\
\hline 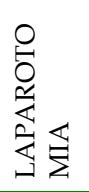 & 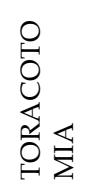 & 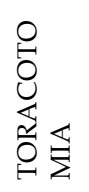 & 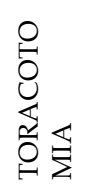 & 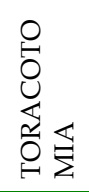 & 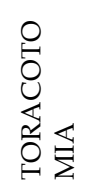 & 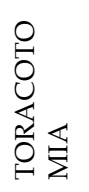 & 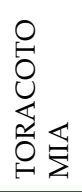 & 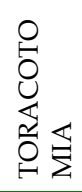 & 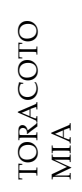 \\
\hline 云 & 云 & 艺 & 文 & 文 & 文 & 文 & 文 & 云 & 文 \\
\hline$\stackrel{\bigcirc}{Z}$ & そ) & $\bar{w}$ & z & ๘ & क & Z & そ) & そ) & ज \\
\hline$\stackrel{\circ}{ }$ & そ) & 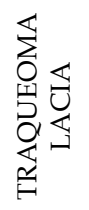 & $\stackrel{\circ}{ }$ & 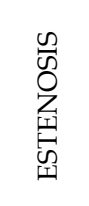 & 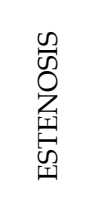 & ○ & Z & 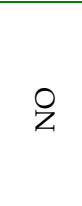 & 尺 \\
\hline 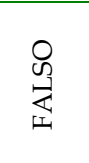 & 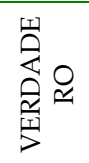 & 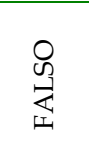 & 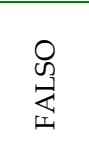 & 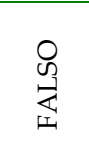 & 怘 & 盈 & 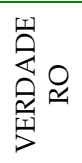 & 怘 & 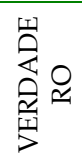 \\
\hline 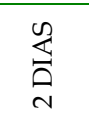 & 㑒就 & $\Rightarrow \stackrel{0}{\Delta}$ & $\circ \sum_{0}^{0}$ & Hi & $\underset{\infty}{\infty}$ & $\underset{\infty}{\infty}$ & in & $\forall \sum^{0}$ & 월 \\
\hline $\begin{array}{l}\frac{F}{8} 8 \\
\frac{8}{8} \dot{8}\end{array}$ & 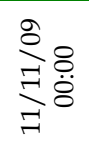 & 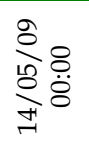 & $\begin{array}{l}\frac{\partial}{2} 8 \\
\text { do } \\
\frac{8}{110}\end{array}$ & 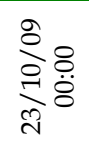 & 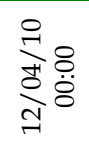 & 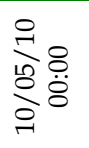 & 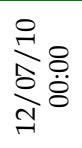 & 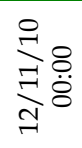 & 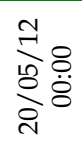 \\
\hline 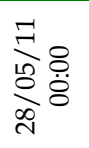 & 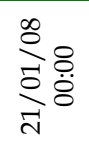 & 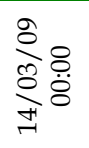 & 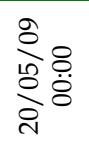 & 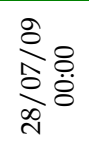 & 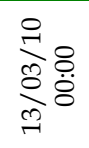 & 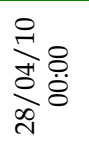 & 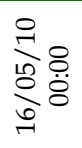 & 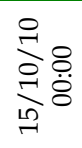 & 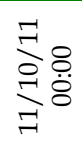 \\
\hline 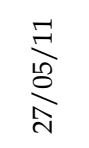 & $\frac{\stackrel{\infty}{\vec{\partial}}}{\vec{\lambda}}$ & 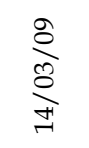 & $\underset{\substack{2 \\
\stackrel{0}{2}}}{\stackrel{0}{a}}$ & $\frac{}{\frac{2}{5}}$ & 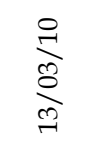 & $\underset{\text { ते }}{\stackrel{2}{\vec{d}}}$ & 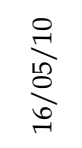 & $\underset{ }{\stackrel{9}{9}}$ & $\underset{\Xi}{\stackrel{\Xi}{g}}$ \\
\hline 号 & 莽 & $\underset{\stackrel{\mathscr{I}}{*}}{ }$ & $\begin{array}{l}\stackrel{0}{0} \\
\ddot{\sim}\end{array}$ & 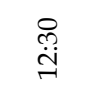 & 㝵 & $\begin{array}{l}\infty \\
\stackrel{\infty}{\hat{n}} \\
\stackrel{i}{n}\end{array}$ & 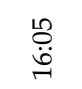 & 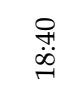 & $\begin{array}{l}\text { 웅 } \\
\text { व }\end{array}$ \\
\hline $\begin{array}{l}\text { 吕 } \\
\text { ف․ }\end{array}$ & 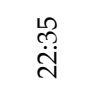 & 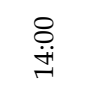 & 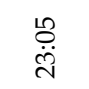 & $\begin{array}{l}\stackrel{\leftrightarrow}{\mathrm{d}} \\
\text { }\end{array}$ & & 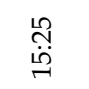 & 兽 & 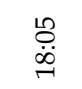 & 吕 \\
\hline 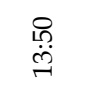 & 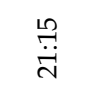 & 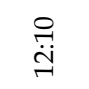 & ஜ̊. & 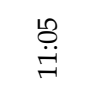 & 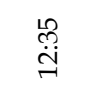 & 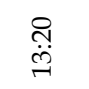 & 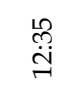 & $\begin{array}{l}\stackrel{\leftrightarrow}{\oplus} \\
\stackrel{\leftrightarrow}{\oplus}\end{array}$ & 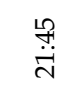 \\
\hline $\begin{array}{l}\text { 年 } \\
\text { త్ }\end{array}$ & 吕 & $\begin{array}{l}\text { \% } \\
\text { ğ }\end{array}$ & 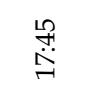 & $\begin{array}{l}\text { 웅 } \\
\text { g! }\end{array}$ & 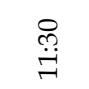 & 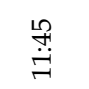 & 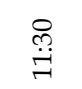 & 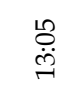 & $\begin{array}{l}\text { ஜn } \\
\stackrel{\leftrightarrow}{\text { N }}\end{array}$ \\
\hline 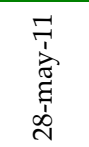 & 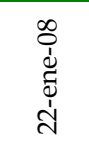 & 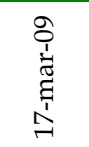 & 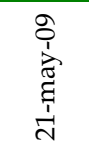 & $\begin{array}{l}0 \\
0 \\
0 \\
0 \\
i \\
i \\
\dot{\lambda}\end{array}$ & 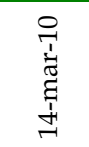 & 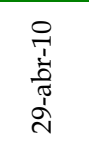 & 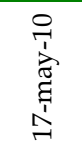 & 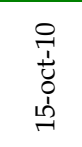 & 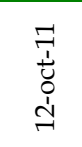 \\
\hline 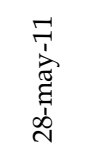 & 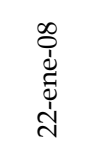 & 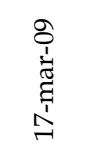 & $\begin{array}{l}\stackrel{0}{0} \\
\stackrel{1}{\leftrightarrows} \\
\stackrel{9}{1} \\
\dot{\lambda}\end{array}$ & $\begin{array}{l}0 \\
0 \\
1 \\
0 \\
⿱ 亠 \\
i \\
\dot{\lambda}\end{array}$ & 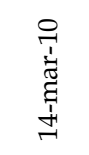 & 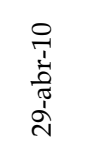 & 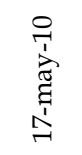 & 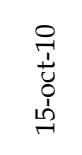 & 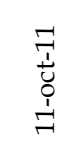 \\
\hline 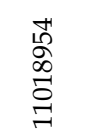 & 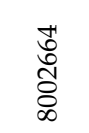 & 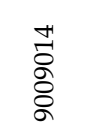 & 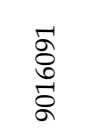 & 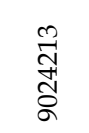 & 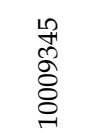 & $\begin{array}{l}\vec{F} \\
10 \\
\stackrel{0}{0} \\
0\end{array}$ & 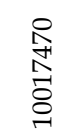 & 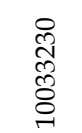 & 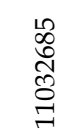 \\
\hline
\end{tabular}




\begin{tabular}{|c|c|c|c|c|c|c|c|}
\hline 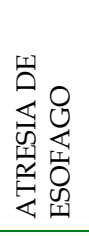 & 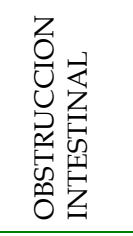 & 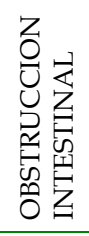 & 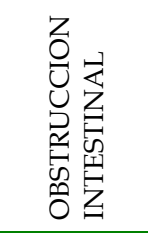 & 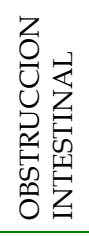 & 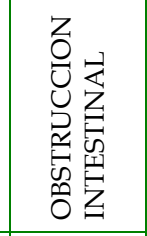 & 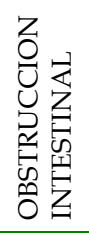 & 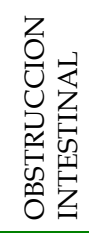 \\
\hline 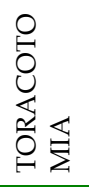 & 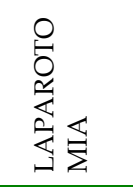 & 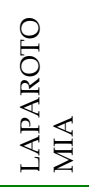 & 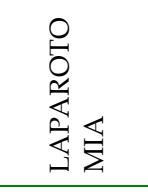 & 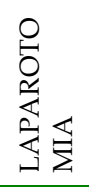 & 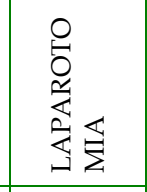 & 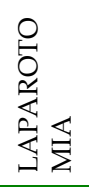 & 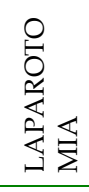 \\
\hline 文 & $\bar{z}$ & Z & 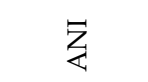 & 妾 & 交 & 艺 & 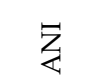 \\
\hline そ) & $\bar{\omega}$ & $\stackrel{\circ}{Z}$ & $\bar{\omega}$ & そ) & $\bar{\omega}$ & $\stackrel{\cap}{Z}$ & そ) \\
\hline$\stackrel{O}{z}$ & 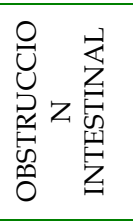 & そ & 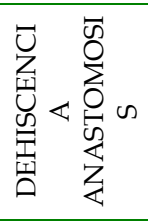 & Z & 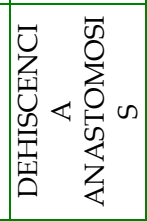 & そ & そ \\
\hline 怘 & 足 & 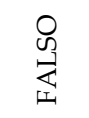 & 怘 & 足 & 怘 & 怘 & 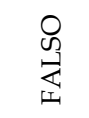 \\
\hline$\sum_{0}^{0}$ & 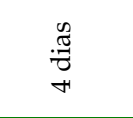 & $\begin{array}{l}\stackrel{n}{\pi} \\
: \pi \\
\sigma\end{array}$ & $\begin{array}{l}\stackrel{n}{\tilde{\Xi}} \\
\stackrel{0}{0} \\
0\end{array}$ & $\begin{array}{l}\stackrel{\mathscr{n}}{\tilde{\Xi}} \\
\stackrel{0}{0}\end{array}$ & 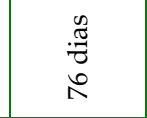 & $\begin{array}{l}\stackrel{0}{\tilde{J}} \\
\text { : }\end{array}$ & $\begin{array}{l}\stackrel{n}{\tilde{J}} \\
\underset{\sim}{\pi}\end{array}$ \\
\hline 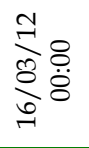 & 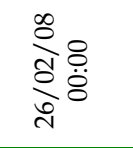 & $\underset{\substack{\infty \\
\hdashline}}{\stackrel{\infty}{0}}$ & 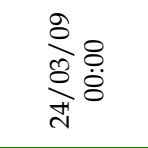 & 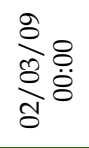 & $\frac{0}{\frac{2}{2}} \frac{8}{0}$ & $\begin{array}{l}\frac{0}{2} 8 \\
\frac{8}{8} \\
\text { वे }\end{array}$ & 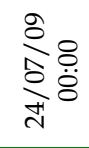 \\
\hline 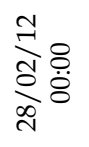 & $\begin{array}{l}\frac{\infty}{2} 8 \\
\frac{0}{0} \\
\frac{0}{0} \\
0 \\
0\end{array}$ & 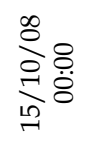 & 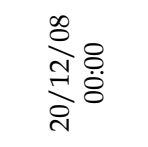 & $\begin{array}{l}\text { 2ेo } \\
\text { के } \\
\text { ते } \\
\text { ते }\end{array}$ & 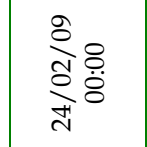 & 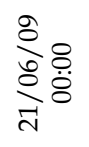 & 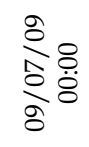 \\
\hline 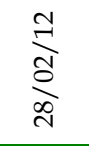 & $\frac{\infty}{\stackrel{\infty}{\infty}}$ & $\underset{\stackrel{\infty}{a}}{\stackrel{\infty}{\rho}}$ & 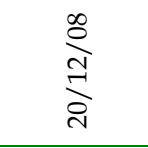 & $\frac{\stackrel{े}{े}}{\vec{े}}$ & $\begin{array}{l}\stackrel{2}{d} \\
\stackrel{d}{d} \\
\text { d }\end{array}$ & $\frac{\frac{o}{o}}{\frac{d}{2}}$ & $\frac{\frac{8}{2}}{\frac{1}{2}}$ \\
\hline 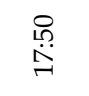 & 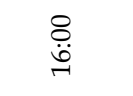 & $\begin{array}{l}\text { @़ } \\
\text { तु }\end{array}$ & 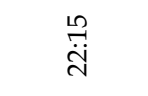 & 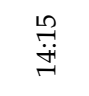 & $\stackrel{\stackrel{\llcorner}{?}}{\stackrel{\sim}{\sim}}$ & 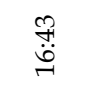 & 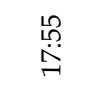 \\
\hline $\begin{array}{l}\stackrel{8}{0} \\
\stackrel{-}{-}\end{array}$ & 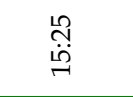 & $\begin{array}{l}\stackrel{\leftrightarrow}{0} \\
\text { ते }\end{array}$ & 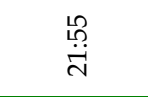 & $\begin{array}{l}\stackrel{0}{0} \\
\stackrel{\leftrightarrow}{\sim}\end{array}$ & $\begin{array}{l}\stackrel{ }{ت} \\
\stackrel{\vec{I}}{-}\end{array}$ & 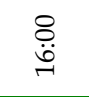 & $\begin{array}{l}\text { तิ } \\
\text { }\end{array}$ \\
\hline 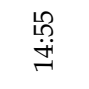 & $\begin{array}{l}\stackrel{0}{0} \\
\stackrel{m}{\emptyset}\end{array}$ & $\begin{array}{l}\stackrel{10}{+} \\
\stackrel{\text { ते }}{0}\end{array}$ & 足 & $\begin{array}{l}\stackrel{\llcorner}{\leftrightarrow} \\
\stackrel{\sim}{ت}\end{array}$ & $\stackrel{\stackrel{P}{\forall}}{\stackrel{H}{*}}$ & 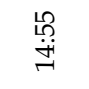 & 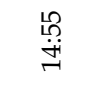 \\
\hline $\begin{array}{l}\stackrel{\leftrightarrow}{\dot{d}} \\
\text { فे }\end{array}$ & 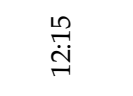 & $\begin{array}{l}\stackrel{\leftrightarrow}{m} \\
\stackrel{\leftrightarrow}{\sigma}\end{array}$ & 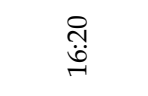 & $\begin{array}{l}\text { ำ } \\
\text { O̊ }\end{array}$ & 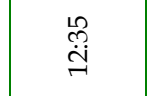 & $\begin{array}{l}\stackrel{\ln }{\ddot{山}} \\
\text { }\end{array}$ & $\begin{array}{l}\stackrel{\leftrightarrow}{0} \\
\ddot{n}\end{array}$ \\
\hline 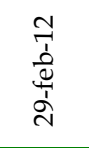 & 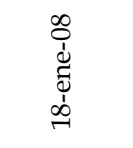 & 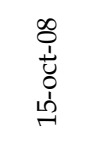 & 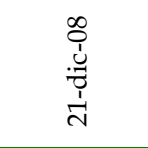 & 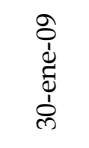 & 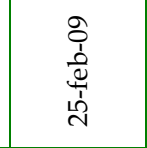 & 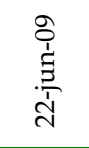 & $\begin{array}{l}0 \\
0 \\
\frac{1}{3} \\
\frac{1}{1} \\
0 \\
\end{array}$ \\
\hline 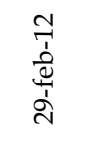 & $\begin{array}{l}\infty \\
\grave{d} \\
\grave{\varpi} \\
\infty \\
\infty\end{array}$ & 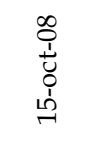 & 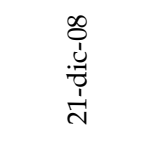 & 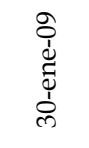 & 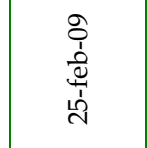 & 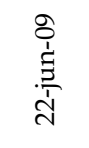 & $\begin{array}{l}0 \\
0 \\
\frac{1}{2} \\
\frac{2}{1} \\
0 \\
0\end{array}$ \\
\hline 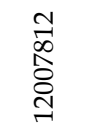 & 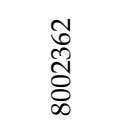 & 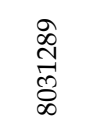 & $\begin{array}{l}D^{\prime} \\
\infty \\
\infty \\
\infty \\
\infty \\
\infty\end{array}$ & 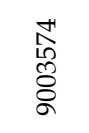 & 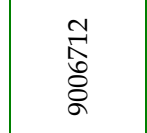 & 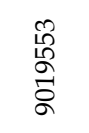 & $\begin{array}{l}\text { ले } \\
\text { तू } \\
\text { ડू }\end{array}$ \\
\hline
\end{tabular}




\begin{tabular}{|c|c|c|c|c|c|c|c|}
\hline 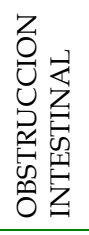 & 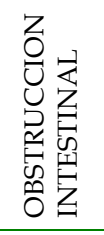 & 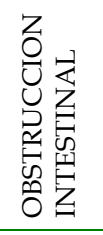 & 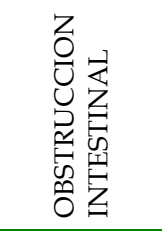 & 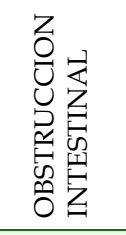 & 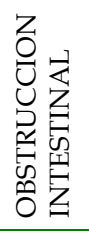 & 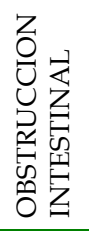 & 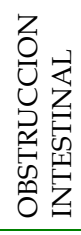 \\
\hline $\begin{array}{l}0 \\
0 \\
0 \\
0 \\
\vdots \\
\vdots \\
\vdots\end{array}$ & 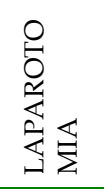 & 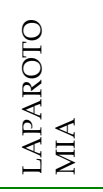 & 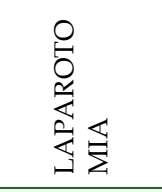 & 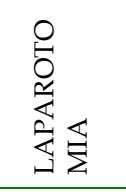 & 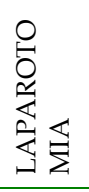 & 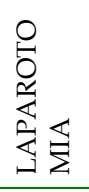 & 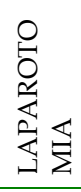 \\
\hline 交 & 文 & 文 & $\bar{z}$ & 云 & 云 & $\begin{array}{l}z \\
\text { Z } \\
\text { D }\end{array}$ & 妾 \\
\hline そ & $\bar{\omega}$ & Z & $\bar{\omega}$ & $\vec{\omega}$ & そ & $\bar{\omega}$ & そ \\
\hline$\stackrel{\circ}{z}$ & $\stackrel{\wp}{Z}$ & ○ & 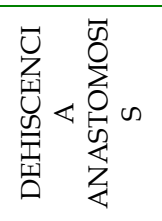 & 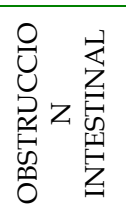 & そ & そ & 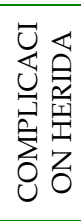 \\
\hline 惫 & 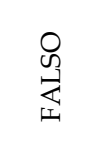 & 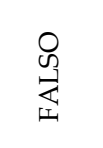 & $\begin{array}{l}\text { O } \\
\text { 崖 }\end{array}$ & 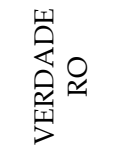 & 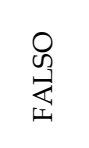 & 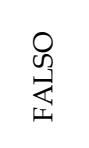 & 怘 \\
\hline 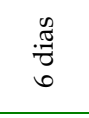 & \begin{tabular}{l}
$\stackrel{g}{0}$ \\
$: \mathbb{7}$ \\
0 \\
\hdashline
\end{tabular} & $\begin{array}{l}\stackrel{n}{\tilde{\Xi}} \\
\text { N }\end{array}$ & 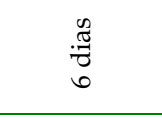 & $\begin{array}{l}\text { g } \\
-\tilde{7} \\
0 \\
0 \\
\end{array}$ & 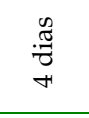 & 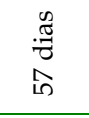 & 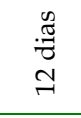 \\
\hline 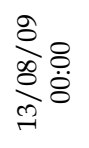 & 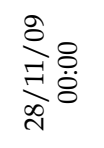 & $\begin{array}{l}\frac{0}{1} 8 \\
\frac{0}{8} \\
\frac{8}{8}\end{array}$ & 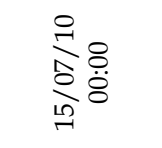 & 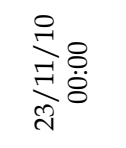 & 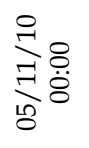 & 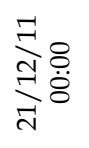 & 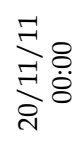 \\
\hline 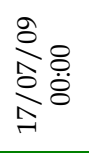 & $\begin{array}{l}\frac{0}{2} 8 \\
\frac{0}{0} \\
\stackrel{0}{0}\end{array}$ & 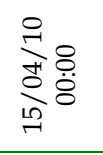 & $\begin{array}{l}\stackrel{0}{8} 8 \\
\stackrel{d}{8}\end{array}$ & 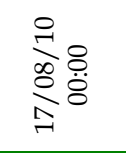 & 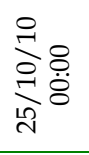 & $\frac{\vec{J}}{\partial} 8$ & 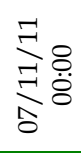 \\
\hline 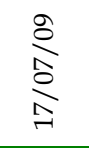 & 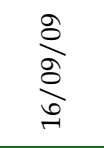 & 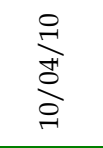 & 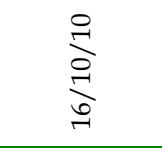 & 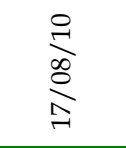 & 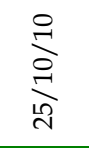 & 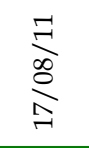 & $\frac{\vec{F}}{8}$ \\
\hline $\begin{array}{l}\stackrel{\mathscr{L}}{\ddot{\infty}} \\
\stackrel{\infty}{\sim}\end{array}$ & $\begin{array}{l}\stackrel{8}{.} \\
\text { in }\end{array}$ & $\begin{array}{l}\text { क़ } \\
\text { ஜ் }\end{array}$ & $\begin{array}{l}\stackrel{\leftrightarrow}{\pi} \\
\stackrel{\text { }}{2}\end{array}$ & $\begin{array}{l}\stackrel{\oplus}{\sigma} \\
\stackrel{\sigma}{0}\end{array}$ & 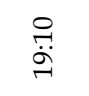 & $\begin{array}{l}\text { ִָ } \\
\text { }\end{array}$ & $\begin{array}{l}\stackrel{\leftrightarrow}{\hat{n}} \\
\text { }\end{array}$ \\
\hline 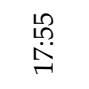 & $\begin{array}{l}\stackrel{\leftrightarrow}{\dddot{m}} \\
\dot{\forall}\end{array}$ & $\begin{array}{l}\stackrel{0}{ } \\
\ddot{0}\end{array}$ & $\begin{array}{l}\text { P } \\
\text { ثें }\end{array}$ & $\begin{array}{l}\stackrel{\leftrightarrow}{ت} \\
\stackrel{\sigma}{0}\end{array}$ & $\begin{array}{l}\stackrel{L}{L} \\
\dot{\infty} \\
\sim\end{array}$ & 邑 & $\begin{array}{l}\infty \\
\stackrel{\tilde{U}}{\text { j}}\end{array}$ \\
\hline & $\begin{array}{l}\stackrel{0}{\dddot{U}} \\
\stackrel{ت}{ }\end{array}$ & $\begin{array}{l}\stackrel{n}{\ddot{H}} \\
\stackrel{d}{0}\end{array}$ & $\begin{array}{l}\text { ஓ̆ } \\
\text { ळे }\end{array}$ & 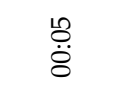 & 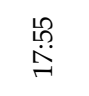 & $\begin{array}{l}\stackrel{\oplus}{\oplus} \\
\stackrel{+}{+}\end{array}$ & $\begin{array}{l}\stackrel{\leftrightarrow}{\sim} \\
\stackrel{\infty}{\sim}\end{array}$ \\
\hline $\begin{array}{l}\stackrel{\ell}{\ell} \\
\stackrel{\leftrightarrow}{+}\end{array}$ & 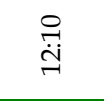 & 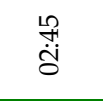 & $\begin{array}{l}\stackrel{\leftrightarrow}{0} \\
\dot{\phi}\end{array}$ & $\begin{array}{l}\text { तิ } \\
\text { ̇̀ }\end{array}$ & $\begin{array}{l}\stackrel{1}{\infty} \\
\stackrel{\leftrightarrow}{\oplus}\end{array}$ & 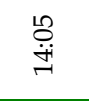 & 㚏 \\
\hline $\begin{array}{l}0 \\
0 \\
\frac{1}{3} \\
0 \\
0 \\
0 \\
0\end{array}$ & 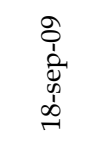 & 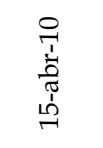 & 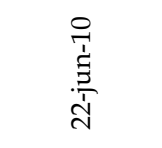 & 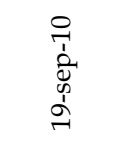 & 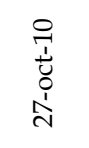 & 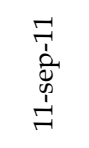 & $\begin{array}{l}7 \\
7 \\
0 \\
0 \\
0 \\
0 \\
0\end{array}$ \\
\hline 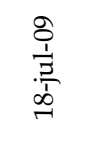 & 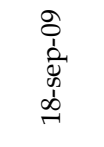 & 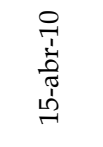 & $\begin{array}{l}\frac{0}{\vec{I}} \\
\frac{\overrightarrow{3}}{\vec{i}}\end{array}$ & 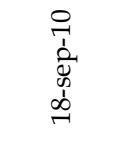 & 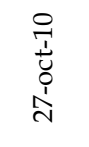 & $\begin{array}{l}\vec{F} \\
\dot{0} \\
\dot{0} \\
. \\
\vec{H}\end{array}$ & 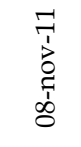 \\
\hline 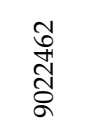 & $\begin{array}{l}\text { N } \\
\text { న̂ } \\
\text { ડે }\end{array}$ & 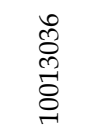 & $\begin{array}{l}\text { ڤ్ } \\
\text { तิ } \\
\text { ઠे }\end{array}$ & 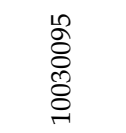 & 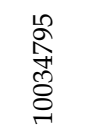 & 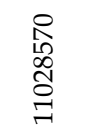 & 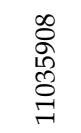 \\
\hline
\end{tabular}




\begin{tabular}{|c|c|c|c|c|c|c|c|c|}
\hline 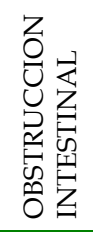 & 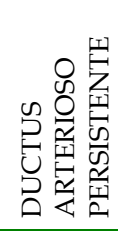 & 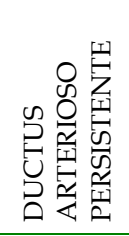 & 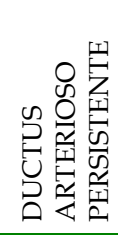 & 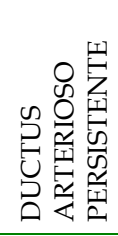 & 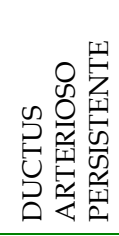 & 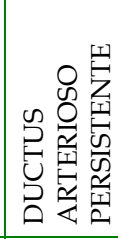 & 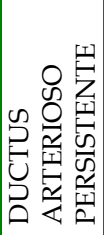 & 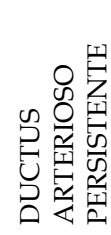 \\
\hline 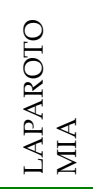 & 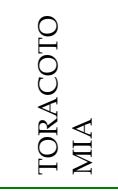 & 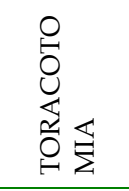 & 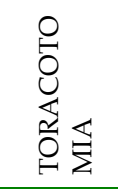 & 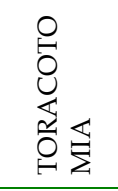 & 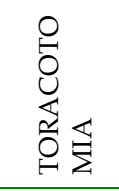 & 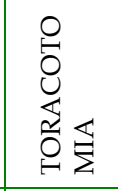 & 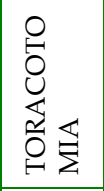 & 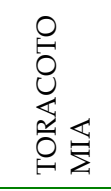 \\
\hline 案 & Z & z & $\begin{array}{l}z \\
\text { Z } \\
0\end{array}$ & Z⿱ 己. & Zu & ż & 妾 & 交 \\
\hline ○ & Z & Z & Z & $\stackrel{\circ}{Z}$ & $\stackrel{\circ}{Z}$ & $\stackrel{\circ}{Z}$ & $\stackrel{\circ}{z}$ & $\stackrel{\circ}{z}$ \\
\hline 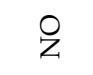 & Z & Z & Z & Z & Z & Z & Z & Z \\
\hline 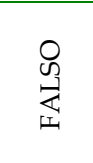 & 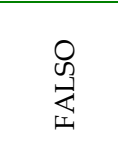 & 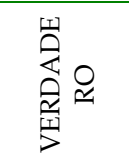 & 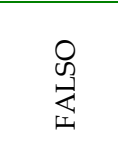 & 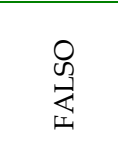 & 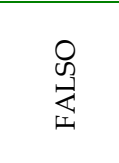 & 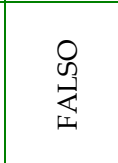 & 足 & 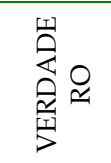 \\
\hline 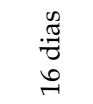 & $\because \frac{\text { SO }}{0}$ & ๓ & 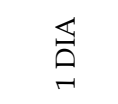 & $8 \stackrel{0}{\Delta}$ & $\approx \stackrel{0}{\Xi}$ & क्ष & 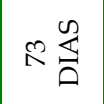 & m这 \\
\hline 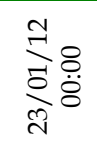 & 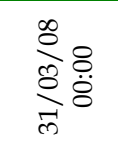 & 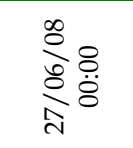 & 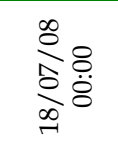 & 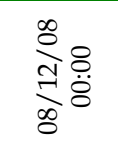 & 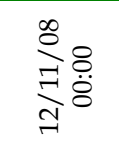 & 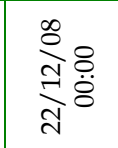 & 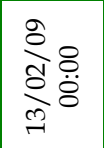 & 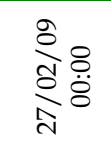 \\
\hline 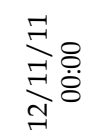 & $\begin{array}{l}\frac{\infty}{2} 8 \\
\frac{0}{2} \\
\stackrel{\sigma}{2}\end{array}$ & 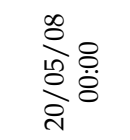 & ${ }_{\substack{0 \\
0}}^{\infty}$ & $\frac{\sum_{0}^{\infty} 8}{0} \frac{0}{0}$ & 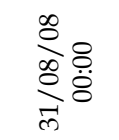 & 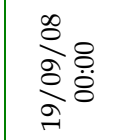 & 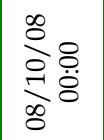 & 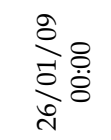 \\
\hline$\underset{ন}{\stackrel{F}{I}}$ & $\frac{\stackrel{\infty}{2}}{\frac{0}{2}}$ & & $\frac{\infty}{\stackrel{\infty}{\circ}}$ & $\frac{\infty}{\stackrel{0}{0}}$ & $\frac{\stackrel{\infty}{\infty}_{\infty}^{\infty}}{\stackrel{\infty}{े}}$ & $\underset{\substack{\infty \\
\stackrel{\infty}{\infty}}}{\stackrel{\infty}{\sim}}$ & $\underset{\frac{1}{2}}{\stackrel{\infty}{\circ}}$ & 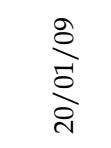 \\
\hline 号 & 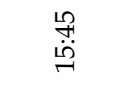 & $\begin{array}{l}0 \\
\stackrel{\infty}{0} \\
\stackrel{\infty}{\sim}\end{array}$ & $\begin{array}{l}\stackrel{\mathscr{L}}{\dddot{m}} \\
\stackrel{+}{\forall}\end{array}$ & $\begin{array}{l}\text { तิ } \\
\text { î }\end{array}$ & 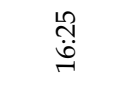 & 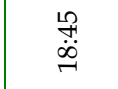 & $\begin{array}{l}\stackrel{\leftrightarrow}{\pi} \\
\stackrel{\infty}{\sim}\end{array}$ & \\
\hline $\begin{array}{l}\stackrel{P}{+} \\
\underset{\forall}{*}\end{array}$ & $\begin{array}{l}\text { 苞 } \\
\text { 苟 }\end{array}$ & 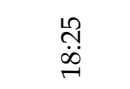 & $\stackrel{\stackrel{\leftrightarrow}{\dddot{\Psi}}}{\underset{\sim}{*}}$ & 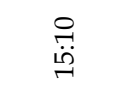 & 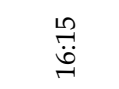 & $\begin{array}{l}\stackrel{\nu}{0} \\
\stackrel{\infty}{\infty}\end{array}$ & $\begin{array}{l}\stackrel{n}{\ddot{\phi}} \\
\stackrel{\vec{\phi}}{\sim}\end{array}$ & $\begin{array}{l}\stackrel{\circ}{\ddot{\sigma}} \\
\text {. }\end{array}$ \\
\hline 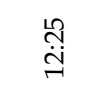 & 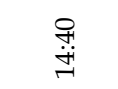 & 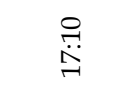 & 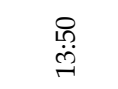 & 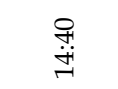 & 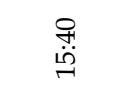 & $\underset{\stackrel{\vartheta}{\ddot{\theta}}}{\stackrel{\rho}{\sim}}$ & 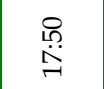 & $\begin{array}{l}\stackrel{0}{0} \\
\dot{\phi} \\
\stackrel{\infty}{\infty}\end{array}$ \\
\hline 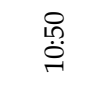 & $\stackrel{\stackrel{2}{\sharp}}{\underset{\sim}{*}}$ & 呙 & ָึં & 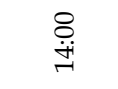 & $\stackrel{\stackrel{P}{\sharp}}{\stackrel{+}{+}}$ & 号 & 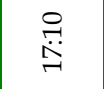 & $\underset{\stackrel{n}{ت}}{\stackrel{n}{ت}}$ \\
\hline 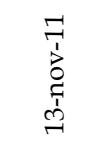 & 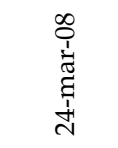 & $\begin{array}{l}\infty \\
0 \\
1 \\
.5 \\
.3 \\
i\end{array}$ & $\begin{array}{l}\infty \\
0 \\
\frac{1}{3} \\
\frac{1}{1} \\
-1\end{array}$ & 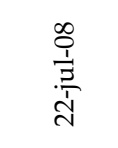 & 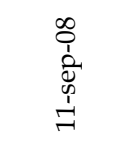 & $\begin{array}{l}\infty \\
0 \\
0 \\
0 \\
0 \\
1 \\
0\end{array}$ & 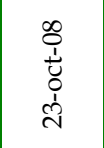 & 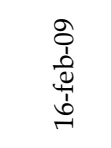 \\
\hline $\begin{array}{l}\overrightarrow{7} \\
\overrightarrow{5} \\
\overline{5} \\
\stackrel{5}{0}\end{array}$ & 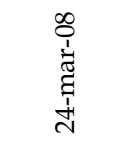 & 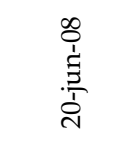 & 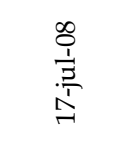 & 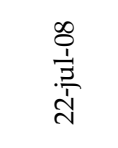 & $\begin{array}{l}\infty \\
0 \\
\dot{0} \\
\dot{0} \\
\dot{d} \\
\vec{f}\end{array}$ & $\begin{array}{l}\infty \\
0 \\
\text { d } \\
0 \\
0 \\
0\end{array}$ & 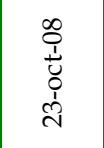 & 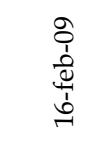 \\
\hline 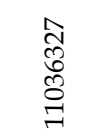 & $\begin{array}{l}\text { के } \\
\text { ळे } \\
\text { ठे }\end{array}$ & 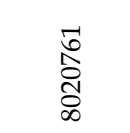 & $\begin{array}{l}\infty \\
\infty \\
0 \\
\widetilde{్} \\
\infty \\
\infty\end{array}$ & 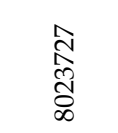 & 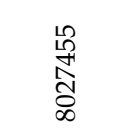 & 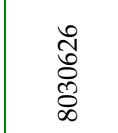 & $\begin{array}{l}\text { శ్ర } \\
\text { స్ } \\
\widetilde{\infty}\end{array}$ & 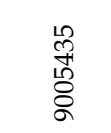 \\
\hline
\end{tabular}




\begin{tabular}{|c|c|c|c|c|c|c|c|c|}
\hline 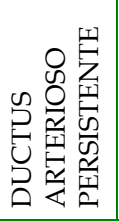 & 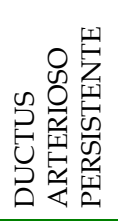 & 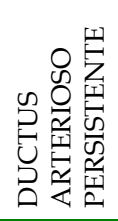 & 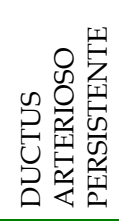 & 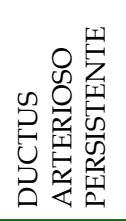 & 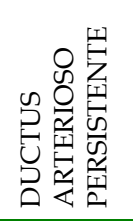 & 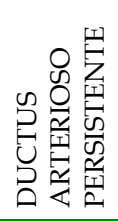 & 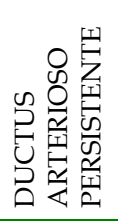 & 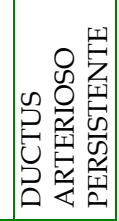 \\
\hline 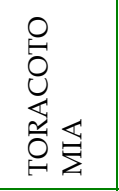 & 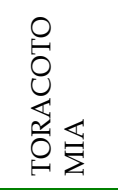 & 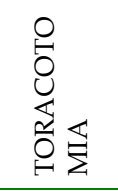 & 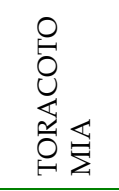 & 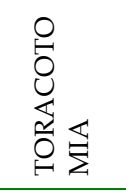 & 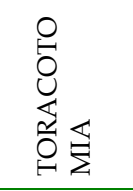 & 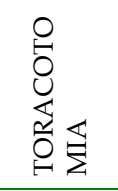 & 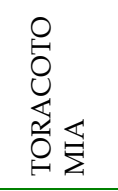 & 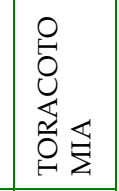 \\
\hline Z⿱ Z & 文 & ż & 云 & $\begin{array}{l}z \\
\text { Z } \\
\vdots\end{array}$ & $\begin{array}{l}z \\
\text { z } \\
\text { D }\end{array}$ & ż & Zu & 妾 \\
\hline 尺े & そ) & そ) & そ) & $\bar{\omega}$ & $\stackrel{\circ}{z}$ & $\stackrel{\circ}{z}$ & 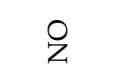 & $\stackrel{\circ}{z}$ \\
\hline ○ & 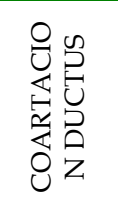 & そ & 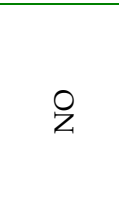 & 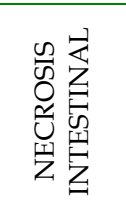 & $\stackrel{\circ}{Z}$ & $\stackrel{O}{Z}$ & 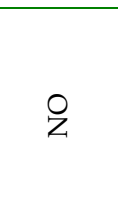 & 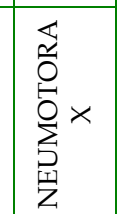 \\
\hline 怘 & 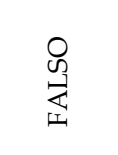 & 怘 & 足 & 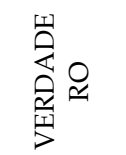 & 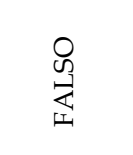 & $\begin{array}{l}0 \\
\text { 足 } \\
\text { 至 }\end{array}$ & 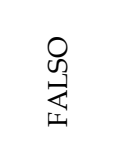 & 怘 \\
\hline กิ & F空 & হ发 & त & $\underset{\infty}{\infty}$ & กิ & $\stackrel{\infty}{\sim} \sum_{0}^{\infty}$ & 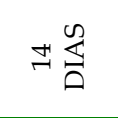 & $\overleftrightarrow{N} \bigsqcup_{0}^{0}$ \\
\hline $\begin{array}{l}\stackrel{0}{2} 8 \\
\frac{\hat{\sigma}}{0} \dot{8} \\
\end{array}$ & 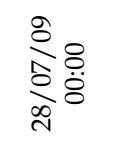 & 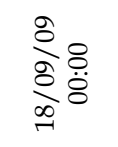 & \begin{tabular}{l}
$\stackrel{0}{0} 8$ \\
†े \\
\multirow{8}{0}{}
\end{tabular} & 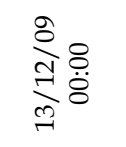 & 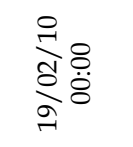 & 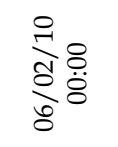 & 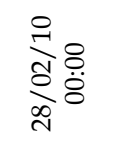 & 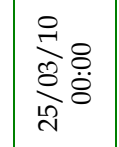 \\
\hline 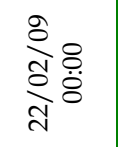 & $\underset{\text { 용 }}{\stackrel{0}{2}}$ & 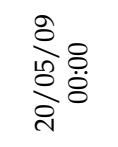 & 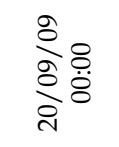 & 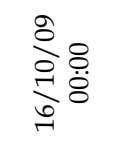 & 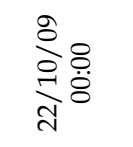 & 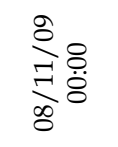 & 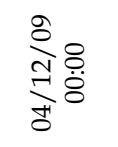 & 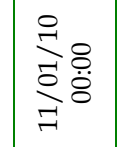 \\
\hline 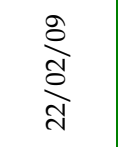 & $\frac{\partial}{\frac{\partial}{2}}$ & 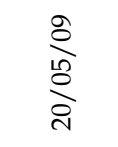 & 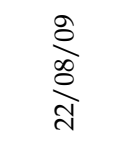 & $\begin{array}{l}\stackrel{o}{\partial} \\
\stackrel{1}{0} \\
\stackrel{-}{-}\end{array}$ & 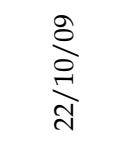 & $\underset{\partial}{\stackrel{\partial}{g}}$ & 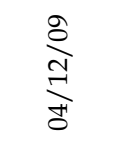 & $\underset{\vec{\partial}}{\stackrel{\ominus}{\ominus}}$ \\
\hline 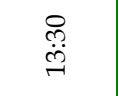 & $\stackrel{\stackrel{n}{n}}{\ddot{\tilde{N}}}$ & $\stackrel{\stackrel{\leftrightarrow}{ت}}{ت}$ & $\begin{array}{l}\stackrel{n}{\ddot{I}} \\
\stackrel{u}{u}\end{array}$ & 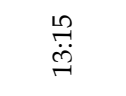 & 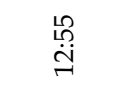 & 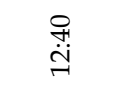 & $\begin{array}{l}\stackrel{n}{\ddot{m}} \\
\stackrel{\ddot{n}}{ }\end{array}$ & 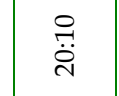 \\
\hline 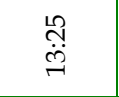 & 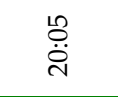 & $\stackrel{\text { ิㅡㅁ }}{\ddot{ت}}$ & 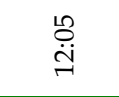 & $\stackrel{\text { }}{\ddot{\ddot{\theta}}}$ & 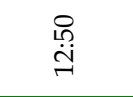 & 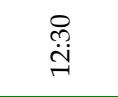 & $\begin{array}{l}\stackrel{\oplus}{\ddot{H}} \\
\stackrel{\ddot{n}}{ }\end{array}$ & 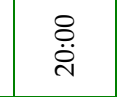 \\
\hline $\begin{array}{l}\stackrel{\leftrightarrow}{.} \\
\ddot{\leftrightarrow}\end{array}$ & $\begin{array}{l}\stackrel{n}{0} \\
\stackrel{a}{g}\end{array}$ & 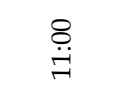 & $\stackrel{\stackrel{\circ}{\sharp}}{\stackrel{H}{ت}}$ & 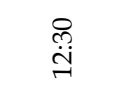 & $\begin{array}{l}\stackrel{\text { }}{\mathrm{j}} \\
\text { }\end{array}$ & 占 & $\begin{array}{l}\stackrel{\leftrightarrow}{\dot{U}} \\
\stackrel{\text { In }}{ }\end{array}$ & $\begin{array}{l}\stackrel{9}{+} \\
\stackrel{9}{2}\end{array}$ \\
\hline 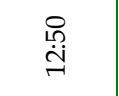 & $\begin{array}{l}\stackrel{1}{0} \\
\stackrel{\infty}{\sim}\end{array}$ & $\frac{10}{\ddot{g}}$ & 号 & 邑 & $\stackrel{\stackrel{\stackrel{P}{7}}{ت}}{ت}$ & 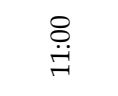 & $\stackrel{\stackrel{0}{0}}{\stackrel{\theta}{\theta}}$ & $\begin{array}{l}\stackrel{9}{4} \\
\stackrel{\infty}{\oplus}\end{array}$ \\
\hline 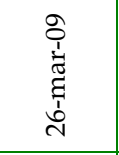 & 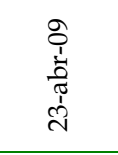 & 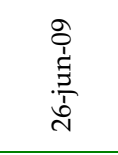 & 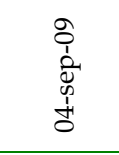 & $\begin{array}{l}8 \\
0 \\
1 \\
0 \\
0 \\
0 \\
8\end{array}$ & 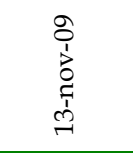 & 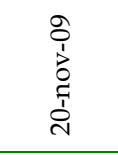 & 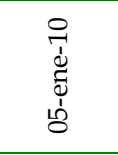 & $\begin{array}{l}\stackrel{0}{1} \\
\dot{d} \\
\grave{d} \\
\dot{N}\end{array}$ \\
\hline 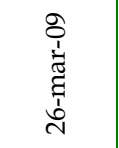 & 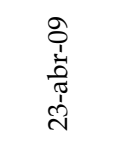 & 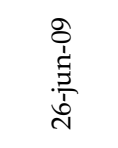 & $\begin{array}{l}\text { oे } \\
\dot{d} \\
\stackrel{0}{0} \\
\text { tे }\end{array}$ & 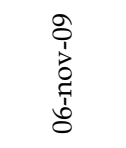 & $\begin{array}{l}0 \\
0 \\
1 \\
0 \\
0 \\
0 \\
\stackrel{1}{\rightleftharpoons}\end{array}$ & $\begin{array}{l}\text { oे } \\
\text { 1े } \\
\text { के } \\
\text { ஸे }\end{array}$ & 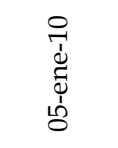 & 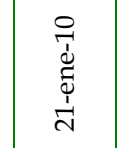 \\
\hline $\begin{array}{l}\text { से } \\
\text { ळे } \\
\text { ळ̆ }\end{array}$ & 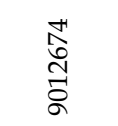 & 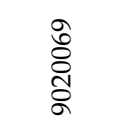 & 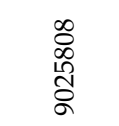 & 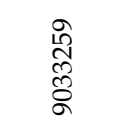 & $\begin{array}{l}\text { पे } \\
\text { ळे } \\
\text { ळू }\end{array}$ & 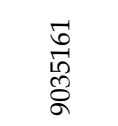 & 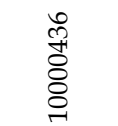 & 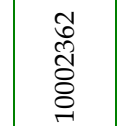 \\
\hline
\end{tabular}




\begin{tabular}{|c|c|c|c|c|c|c|c|c|}
\hline 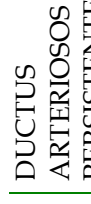 & 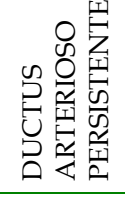 & 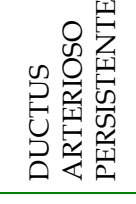 & 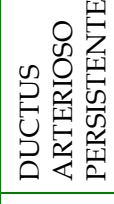 & 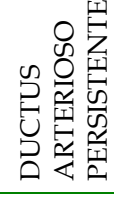 & 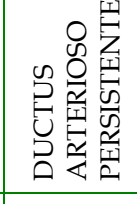 & 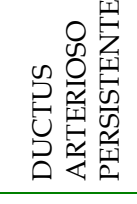 & 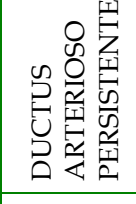 & 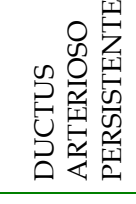 \\
\hline 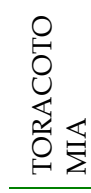 & 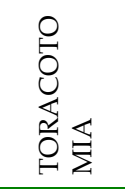 & 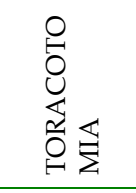 & 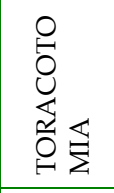 & 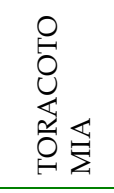 & 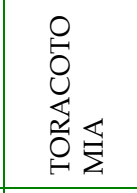 & 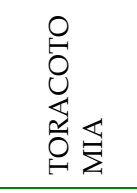 & 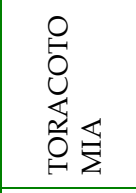 & 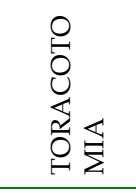 \\
\hline $\bar{z}$ & $\stackrel{z}{\mathrm{z}}$ & 交 & z & $\stackrel{z}{\mathrm{z}}$ & 交 & 交 & 交 & z \\
\hline$\stackrel{8}{z}$ & $\stackrel{\circ}{z}$ & $\stackrel{\circ}{z}$ & \& & $\stackrel{\circ}{z}$ & $\stackrel{\circ}{2}$ & $\stackrel{\circ}{2}$ & $\stackrel{\circ}{z}$ & $\stackrel{8}{2}$ \\
\hline$\stackrel{8}{2}$ & $\stackrel{\gtrless}{z}$ & z & $\stackrel{2}{z}$ & そ & $\stackrel{8}{z}$ & $\stackrel{q}{z}$ & $\stackrel{̊}{z}$ & $\stackrel{̊}{z}$ \\
\hline 褐 & 品 & 兽 & 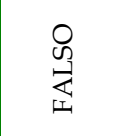 & 孚 & $\begin{array}{l}0 \\
\text { 离 } \\
\text { 离 }\end{array}$ & 孚 & 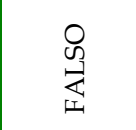 & 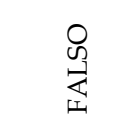 \\
\hline ma & 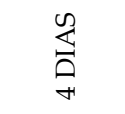 & 嶽 & 和僐 & 웜 & $\vec{m}$ & 路 & $n$ & 路 \\
\hline 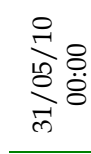 & 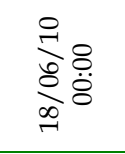 & 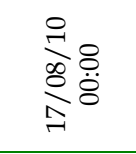 & 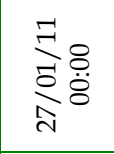 & 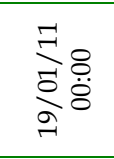 & 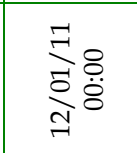 & $\begin{array}{l}\text { Fo } \\
\text { Fo } \\
\text { o. }\end{array}$ & 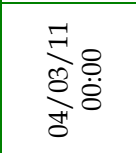 & 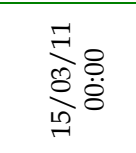 \\
\hline 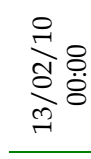 & 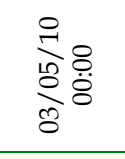 & 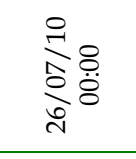 & 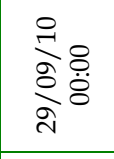 & 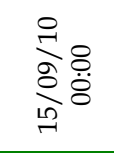 & 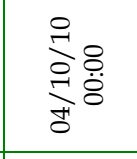 & 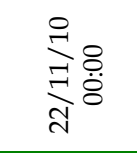 & 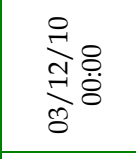 & $\begin{array}{l}\text { 궁여 } \\
\text { 충 }\end{array}$ \\
\hline స్ํํ & 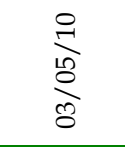 & 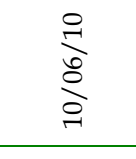 & $\begin{array}{l}\text { के } \\
\text { के } \\
\text { के }\end{array}$ & $\begin{array}{l}9 \\
\frac{9}{9} \\
\end{array}$ & $\begin{array}{l}0 \\
\frac{1}{2} \\
\frac{1}{8}\end{array}$ & 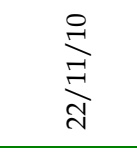 & $\frac{\sum_{0}^{2}}{\frac{\pi}{8}}$ & 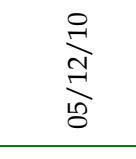 \\
\hline 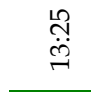 & 夺 & 量 & 营 & $\begin{array}{l}\text { 总 } \\
\text { di }\end{array}$ & : & 곡 & 㿣 & 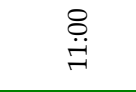 \\
\hline 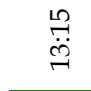 & 虽 & 卤 & 兽 & 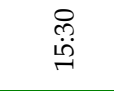 & 岁 & 冚 & 昜 & 昜 \\
\hline 沀 & $\begin{array}{l}\text { 籴 } \\
\text { d. }\end{array}$ & $\begin{array}{l}\text { 是 } \\
\text { 晋 }\end{array}$ & 虽 & 䧽 & 品 & $\stackrel{0}{\stackrel{D}{\#}}$ & 爭 & 兽 \\
\hline 迅 & 量 & 釋 & $\begin{array}{l}\frac{2}{2} \\
\dot{D}\end{array}$ & Fิ & 㟔 & $\begin{array}{l}\frac{l}{9} \\
\text { of }\end{array}$ & $\frac{10}{P}$ & $\begin{array}{l}9 \\
\frac{9}{8}\end{array}$ \\
\hline 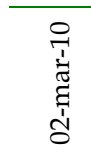 & 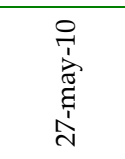 & $\begin{array}{l}\text { वे. } \\
\text { d. } \\
\text { dै } \\
\text { dै }\end{array}$ & 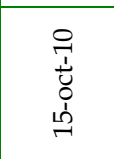 & 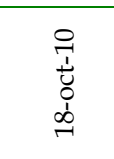 & 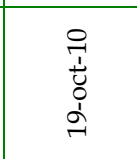 & 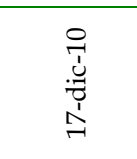 & 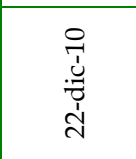 & 买 \\
\hline 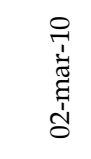 & 蒿 & 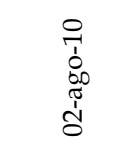 & 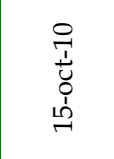 & 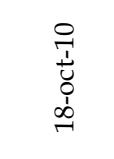 & 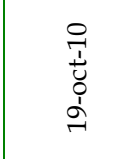 & $\begin{array}{l}\text { 营 } \\
\text { 竞 }\end{array}$ & 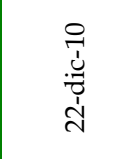 & 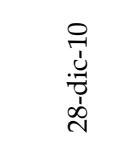 \\
\hline 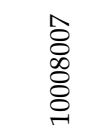 & 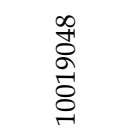 & 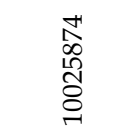 & 䍞 & 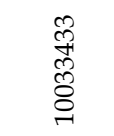 & 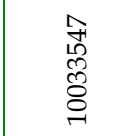 & 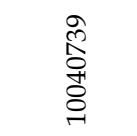 & 胥 & 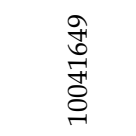 \\
\hline
\end{tabular}




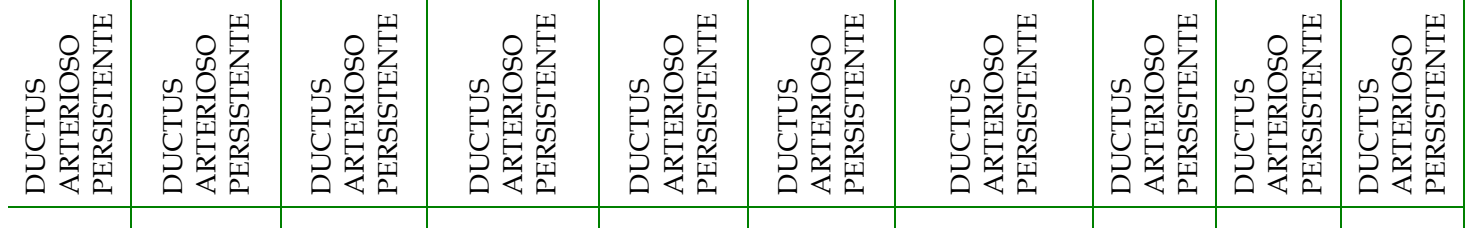

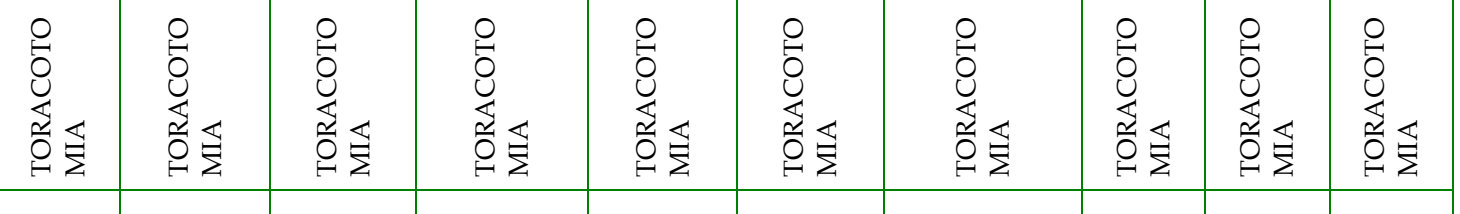

\begin{tabular}{|c|c|c|c|c|c|c|c|c|}
\hline 孞 & 㭉 & 文 & 文 & 妾 & 文 & $\underset{4}{\mathrm{Z}}$ & Z & $\underset{4}{Z}$ \\
\hline ฉ & そ & z & そ & $\stackrel{\gtrless}{z}$ & z & $\stackrel{\gtrless}{z}$ & \& & そ \\
\hline
\end{tabular}

\begin{tabular}{|c|c|c|c|c|c|c|c|c|}
\hline ○ & 8 & 8 & ৪ & প & ৪ & ○ & 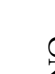 & 8 \\
\hline
\end{tabular}

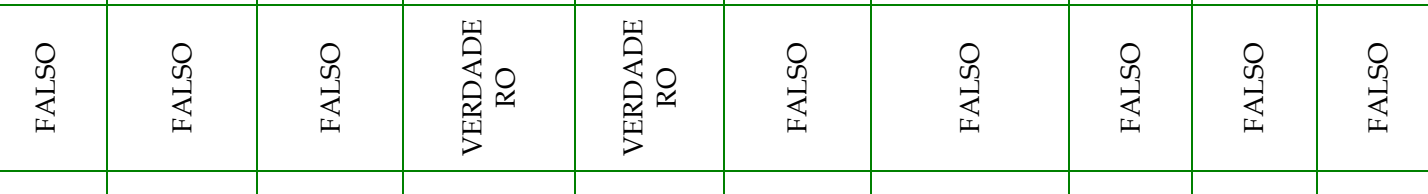

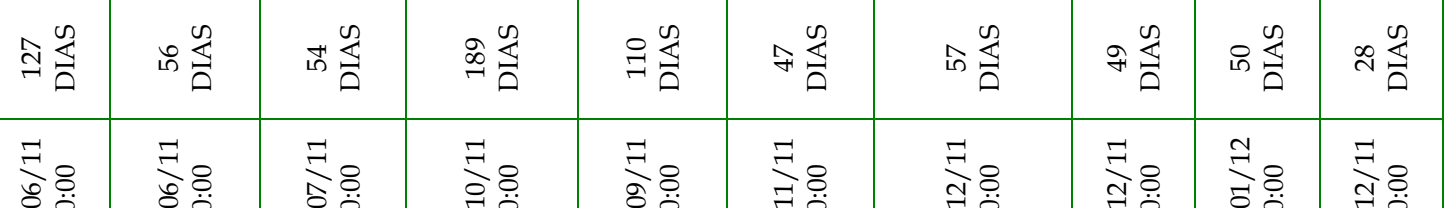

\begin{tabular}{|c|c|c|c|c|c|c|c|c|c|}
\hline $\begin{array}{l}\text { Fे } \\
\text { \&̊ } \\
\text { ¿े }\end{array}$ & 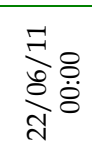 & 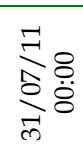 & 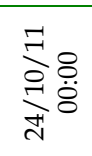 & $\begin{array}{l}\text { Fo } \\
\text { o. } \\
\text { o. } \\
\text { d. }\end{array}$ & $\underset{\text { ㅇㅎㅇ }}{\vec{J}}$ & 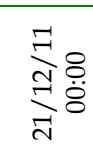 & 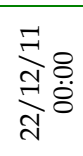 & 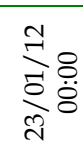 & 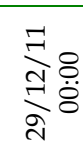 \\
\hline 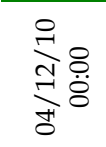 & 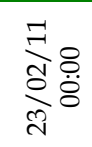 & 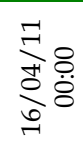 & 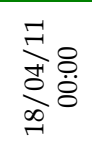 & 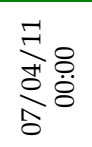 & 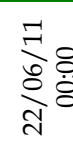 & $\frac{7}{\frac{7}{0} \%}$ & 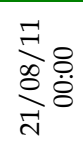 & $\begin{array}{l}\text { Fo } \\
\text { 용 } \\
\text { ते }\end{array}$ & 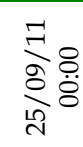 \\
\hline
\end{tabular}

\begin{tabular}{|c|c|c|c|c|c|c|c|c|c|}
\hline$\frac{\underset{d}{g}}{\frac{g}{d}}$ & 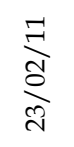 & 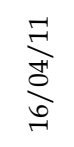 & 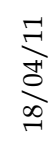 & $\underset{\frac{D}{8}}{\overrightarrow{8}}$ & \begin{tabular}{l}
$\overrightarrow{7}$ \\
$\delta$ \\
\multirow{2}{*}{}
\end{tabular} & 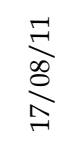 & $\begin{array}{l}\vec{\nabla} \\
\stackrel{0}{2} \\
\vec{\lambda}\end{array}$ & $\frac{\vec{d}}{\Delta} \frac{d}{d}$ & $\frac{\vec{g}}{\Delta}$ \\
\hline స్ & $\begin{array}{l}\text { I্ } \\
\stackrel{\sim}{\sim}\end{array}$ & 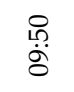 & 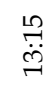 & $\underset{\forall}{\stackrel{n}{*}}$ & 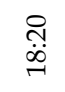 & $\underset{\substack{\infty \\
\sim}}{ }$ & 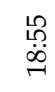 & 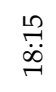 & 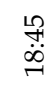 \\
\hline 兄 & 亲 & $\begin{array}{l}\frac{2}{0} \\
\stackrel{5}{0}\end{array}$ & 号 & 猋 & 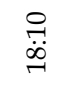 & 总 & 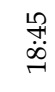 & $\stackrel{P}{\stackrel{P}{\oplus}}$ & 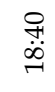 \\
\hline 导 & & 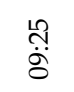 & 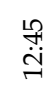 & 卢 & 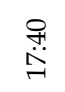 & 哭 & $\begin{array}{l}\mathscr{D} \\
\stackrel{D}{\infty}\end{array}$ & 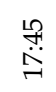 & $\begin{array}{l}\mathscr{D} \\
\stackrel{\infty}{\oplus}\end{array}$ \\
\hline 吕 & $\begin{array}{l}\text { 㝵 } \\
\text { d. }\end{array}$ & 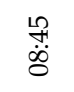 & 号 & 总 & 号 & 吕 & 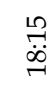 & న్ని & 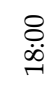 \\
\hline 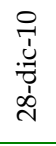 & $\begin{array}{l}7 \overrightarrow{7} \\
\frac{1}{5} \\
\frac{1}{0}\end{array}$ & 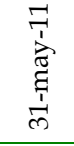 & 竞 & $\begin{array}{l}\overrightarrow{7} \\
\frac{7}{3} \\
\frac{1}{d}\end{array}$ & $\begin{array}{l}\overrightarrow{7} \\
\frac{7}{3} \\
\frac{3}{3}\end{array}$ & 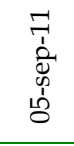 & 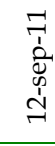 & $\begin{array}{l}\overrightarrow{7} \\
\mathbf{0} \\
0 \\
0\end{array}$ & $\begin{array}{l}\vec{F} \\
\dot{0} \\
\dot{I}\end{array}$ \\
\hline 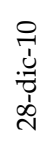 & 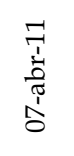 & 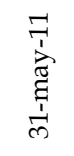 & $\begin{array}{l}\overrightarrow{7} \\
\frac{\overrightarrow{1}}{5} \\
\frac{7}{5}\end{array}$ & 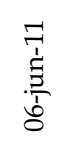 & 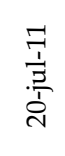 & 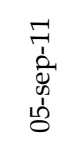 & 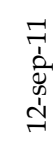 & $\begin{array}{l}\overrightarrow{7} \\
\mathbf{0} \\
0 \\
0\end{array}$ & $\begin{array}{l}\overrightarrow{7} \\
\mathbf{t} \\
\dot{+} \\
+\end{array}$ \\
\hline 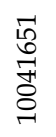 & 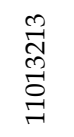 & 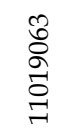 & $\begin{array}{l}\text { वे } \\
\text { Dे } \\
\vec{\exists}\end{array}$ & 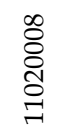 & $\begin{array}{l}\text { प艹 } \\
\text { dै } \\
\text { dै }\end{array}$ & $\begin{array}{l}\text { ते } \\
\text { స్ }\end{array}$ & 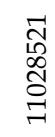 & 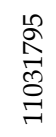 & 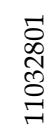 \\
\hline
\end{tabular}




\begin{tabular}{|c|c|c|c|c|c|c|c|c|}
\hline 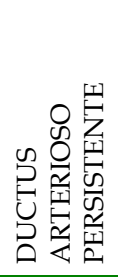 & 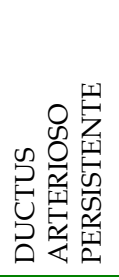 & 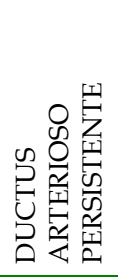 & 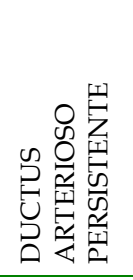 & 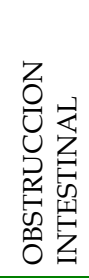 & 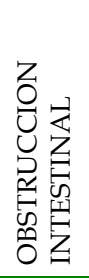 & 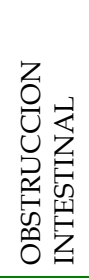 & 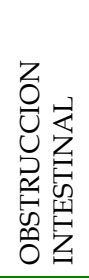 & 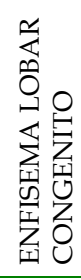 \\
\hline 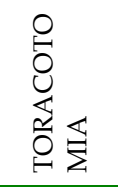 & 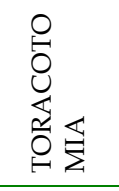 & 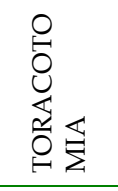 & 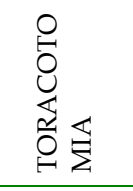 & 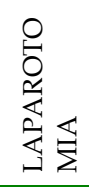 & 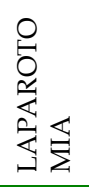 & 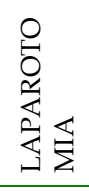 & 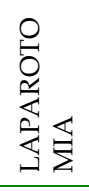 & 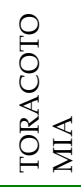 \\
\hline ż & 文 & 文 & 玄 & 交 & 云 & 云 & 云 & 云 \\
\hline$\stackrel{\circ}{Z}$ & そ) & そ) & $\stackrel{\circ}{z}$ & $\bar{\omega}$ & そ & 尺े & 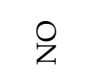 & 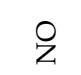 \\
\hline$\stackrel{̊}{Z}$ & そ) & そ) & $\stackrel{\circ}{z}$ & 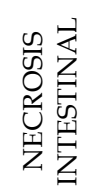 & 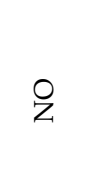 & 尺 & 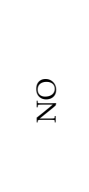 & そ) \\
\hline 怘 & 足 & 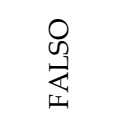 & 怘 & 足 & 足 & 足 & 怘 & 怘 \\
\hline 的起 & 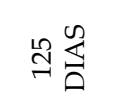 & 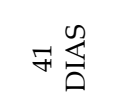 & Hت & ৯ & $\underset{\neg}{\overleftrightarrow{\Xi}}$ & 盛 & $\begin{array}{l}\stackrel{0}{\Delta} \\
\text { Nats }\end{array}$ & ${ }_{\substack{\Delta \\
\vec{A}}}^{0}$ \\
\hline 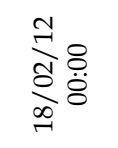 & 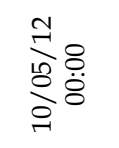 & 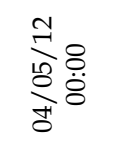 & $\begin{array}{l}\text { Jैo } \\
\text { o. } \\
\underset{7}{7}\end{array}$ & 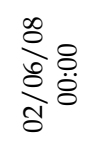 & 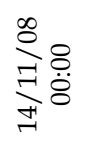 & 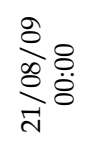 & 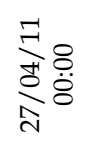 & $\begin{array}{l}\text { के } \\
\text { ते } \\
\text { ते } \\
\text { के }\end{array}$ \\
\hline 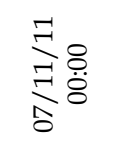 & 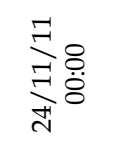 & 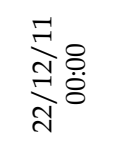 & $\begin{array}{l}\text { Fo } \\
\text { సิ } \\
\text { নે }\end{array}$ & 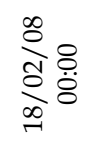 & 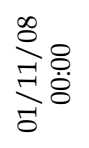 & $\frac{o}{\frac{0}{0}} \frac{0}{8}$ & 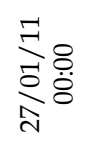 & 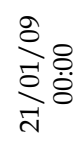 \\
\hline$\underset{\text { 공 }}{\vec{B}}$ & $\underset{⿱ 乛}{\stackrel{\Xi}{\Xi}}$ & 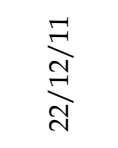 & 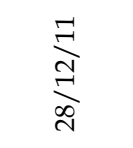 & $\frac{\stackrel{\infty}{\curvearrowright}}{\stackrel{0}{ٍ}}$ & 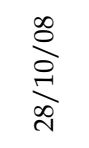 & $\frac{\frac{8}{2}}{\frac{1}{8}}$ & $\frac{\vec{\Xi}}{\stackrel{\Xi}{a}}$ & $\frac{\infty}{\stackrel{\infty}{I}}$ \\
\hline 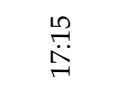 & 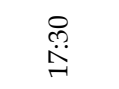 & & $\begin{array}{l}\stackrel{\leftrightarrow}{\sigma} \\
\stackrel{\sigma}{\sigma}\end{array}$ & $\begin{array}{l}\text { \&̊․ } \\
\text { }\end{array}$ & 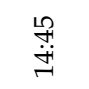 & $\begin{array}{l}\text { Oे } \\
\stackrel{+}{+}\end{array}$ & $\underset{\stackrel{f}{+}}{\stackrel{f}{*}}$ & 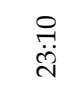 \\
\hline 苛 & 突 & 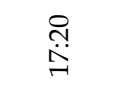 & $\begin{array}{l}\stackrel{2}{\circ} \\
\ddot{\sigma}\end{array}$ & $\begin{array}{l}\text { مٌ } \\
\stackrel{\leftrightarrow}{\sigma}\end{array}$ & $\stackrel{\mathscr{L}}{\stackrel{\sim}{*}}$ & 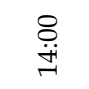 & 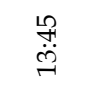 & 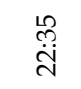 \\
\hline $\begin{array}{l}\text { ف̊ } \\
\text { }\end{array}$ & 号 & 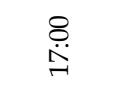 & 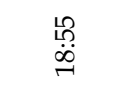 & $\stackrel{\stackrel{\bullet}{0}}{\dot{\infty}}$ & 苂 & 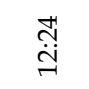 & 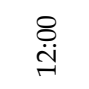 & ণิָ \\
\hline 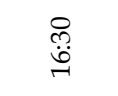 & 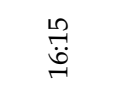 & 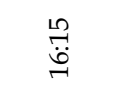 & $\begin{array}{l}\stackrel{T}{1} \\
\dot{\infty} \\
\rightarrow\end{array}$ & ڤึి & $\begin{array}{l}\text { ণ़े } \\
\ddot{8}\end{array}$ & 吊 & $\stackrel{\text { ڤٌ }}{\ddot{ت}}$ & 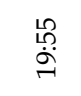 \\
\hline $\begin{array}{l}\vec{i} \\
: \vec{i} \\
\dot{i} \\
\vec{\lambda}\end{array}$ & 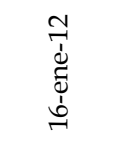 & 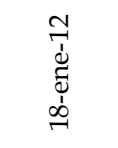 & 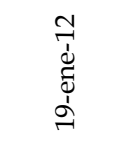 & 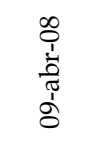 & $\begin{array}{l}\infty \\
\vdots \\
\vdots \\
0 \\
\vdots \\
0 \\
0\end{array}$ & 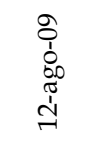 & $\begin{array}{l}\overrightarrow{7} \\
\frac{7}{0} \\
\frac{\pi}{10} \\
\frac{1}{0}\end{array}$ & 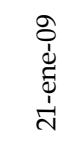 \\
\hline $\begin{array}{l}\vec{i} \\
: \vec{i} \\
\dot{i} \\
\dot{\lambda}\end{array}$ & 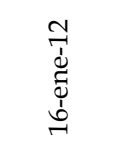 & 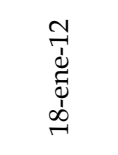 & 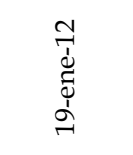 & 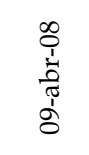 & $\begin{array}{l}\infty \\
0 \\
1 \\
0 \\
0 \\
1 \\
0\end{array}$ & 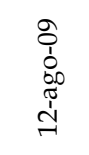 & $\begin{array}{l}\overrightarrow{7} \\
\frac{7}{5} \\
\stackrel{5}{0} \\
0\end{array}$ & $\begin{array}{l}\stackrel{9}{0} \\
\dot{d} \\
\grave{d} \\
\grave{N}\end{array}$ \\
\hline $\begin{array}{l}\infty \\
\stackrel{2}{\circ} \\
\stackrel{+}{0} \\
\stackrel{్}{\sigma}\end{array}$ & 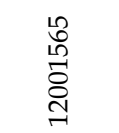 & 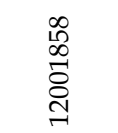 & 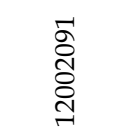 & $\begin{array}{l}\text { ڤి } \\
\text { הี } \\
\text { 亏े }\end{array}$ & 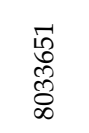 & 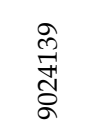 & 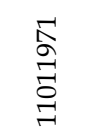 & 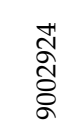 \\
\hline
\end{tabular}




\begin{tabular}{|c|c|c|c|c|c|c|c|c|}
\hline 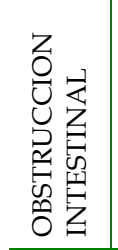 & 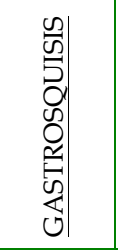 & 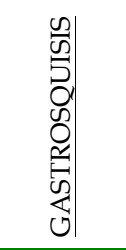 & $\begin{array}{l}0 \\
0 \\
5 \\
0 \\
0 \\
0 \\
0 \\
0 \\
0\end{array}$ & $\begin{array}{l}0 \\
0 \\
5 \\
0 \\
0 \\
0 \\
0 \\
0 \\
0\end{array}$ & 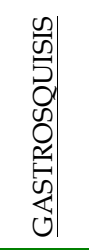 & 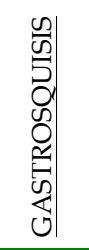 & 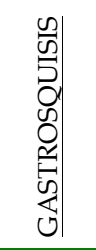 & 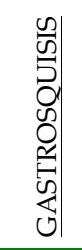 \\
\hline 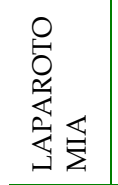 & 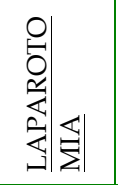 & 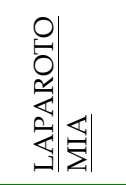 & 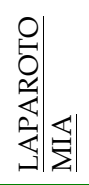 & 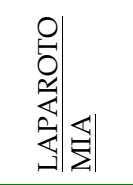 & 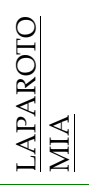 & 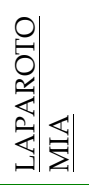 & 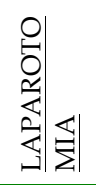 & 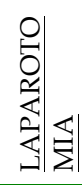 \\
\hline 文 & $\begin{array}{l}\mathrm{Z} \\
\mathrm{b} \\
\mathrm{b}\end{array}$ & $\bar{z}$ & 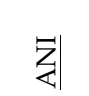 & 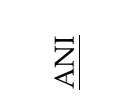 & $\underset{\gtrless}{ }$ & 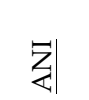 & 艺 & 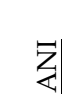 \\
\hline क & जा & ๘) & $\begin{array}{l}O \\
Z\end{array}$ & ๘) & O & ๘) & O & O \\
\hline 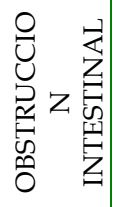 & 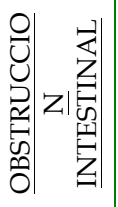 & 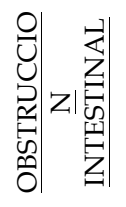 & O) & 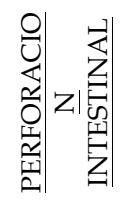 & : & 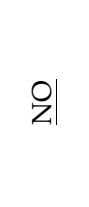 & $\begin{array}{l}\mathbf{Z} \\
\mathbf{Z}\end{array}$ & O \\
\hline 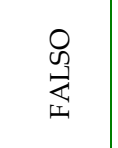 & 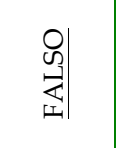 & 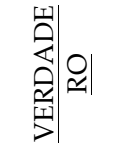 & 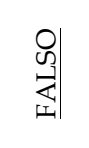 & 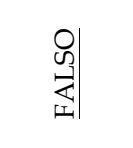 & 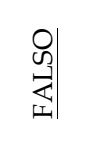 & 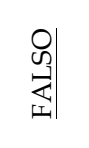 & $\underset{0}{0}$ & 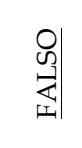 \\
\hline ल & $\vec{\nabla}|\mathfrak{a}|$ & $\stackrel{\infty}{\sim}|\stackrel{\infty}{\overrightarrow{0}}|$ & 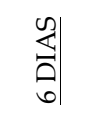 & $\vec{m}|\stackrel{\Xi}{\vec{D}}|$ & $\begin{array}{l}\stackrel{0}{\vdots} \\
\text { in }\end{array} \mid$ & 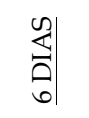 & $\underset{\mid \mathbb{S}}{\stackrel{0}{\mid}}$ & 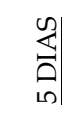 \\
\hline 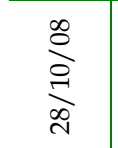 & 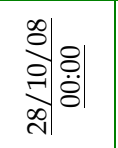 & 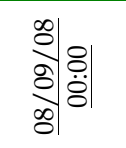 & 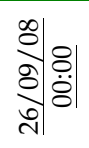 & 突於 & 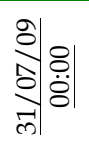 & 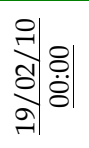 & 彥| & 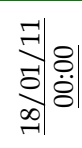 \\
\hline$\stackrel{\infty}{\stackrel{\infty}{2}}$ & 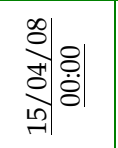 & 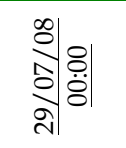 & 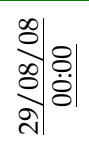 & 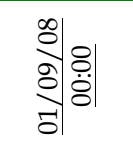 & 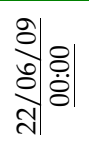 & 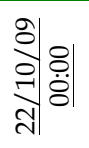 & 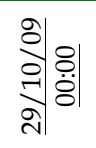 & 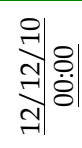 \\
\hline$\stackrel{\infty}{\stackrel{\infty}{2}}$ & 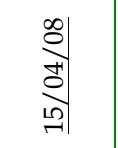 & 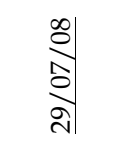 & 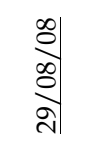 & $\begin{array}{l}\text { के } \\
\text { के } \\
\text { के }\end{array}$ & $\begin{array}{l}\text { Dे } \\
\text { oे } \\
\text { तो } \\
\text { तो }\end{array}$ & 究 & خे & 워 \\
\hline 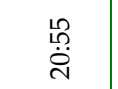 & 它 & 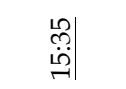 & 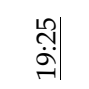 & 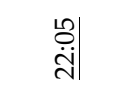 & 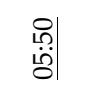 & 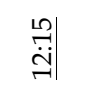 & 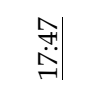 & 吞 \\
\hline 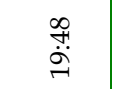 & $\stackrel{\text { 孚 }}{\underset{*}{*}}$ & 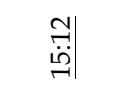 & 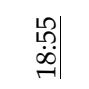 & $\stackrel{\stackrel{\llcorner}{:}}{\stackrel{\leftrightarrow}{N}}$ & 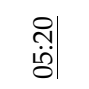 & 哭 & 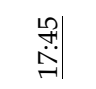 & 옴 \\
\hline $\begin{array}{l}\text { 華 } \\
\text { 슨 }\end{array}$ & 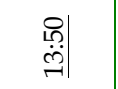 & 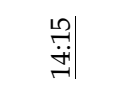 & 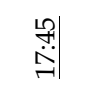 & $\begin{array}{l}\text { @े } \\
\stackrel{\leftrightarrow}{0}\end{array}$ & 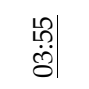 & $\stackrel{\text { : }}{\stackrel{\vec{ت}}{\mid}}$ & 号 & 胃| \\
\hline 苗 & 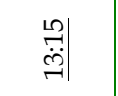 & 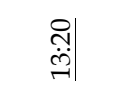 & $\begin{array}{c}\text { กี๊ } \\
\dot{\hat{n}}\end{array}$ & $\begin{array}{c}\stackrel{0}{0} \\
\stackrel{\sim}{\natural}\end{array}$ & $\begin{array}{c}\widetilde{\omega} \\
\ddot{\delta} \mid\end{array}$ & $\begin{array}{l}\stackrel{\circ}{\llcorner} \\
\dot{g} \\
\dot{g}\end{array}$ & 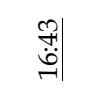 & 号| \\
\hline 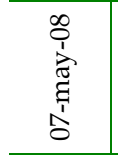 & 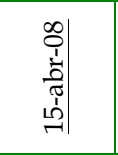 & $\begin{array}{l}0 \\
0 \\
\vdots \\
\vdots \\
\vdots \\
\text { הे }\end{array}$ & 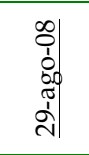 & $\begin{array}{l}0 \\
0 \\
\vdots \\
\vdots \\
0 \\
\vdots \\
\vdots\end{array}$ & 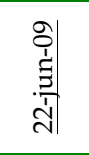 & 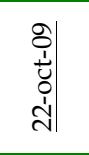 & 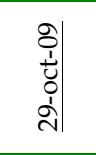 & 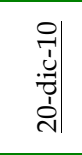 \\
\hline 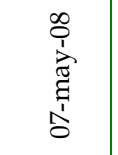 & 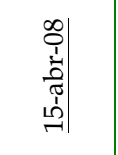 & 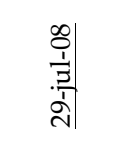 & \begin{tabular}{c|c} 
& 0 \\
$\vdots$ \\
$\vdots$ \\
$\vdots$ \\
$\vdots$ \\
$\vdots$
\end{tabular} & $\begin{array}{l}0 \\
0 \\
\vdots \\
0 \\
0 \\
0 \\
\vdots \\
0\end{array}$ & 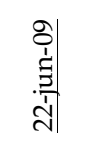 & 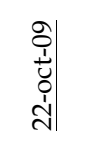 & 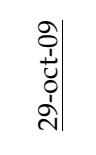 & 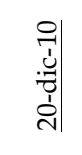 \\
\hline $\begin{array}{l}\stackrel{0}{0} \\
\stackrel{0}{0} \\
\stackrel{1}{0} \\
\stackrel{0}{0}\end{array}$ & 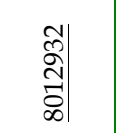 & 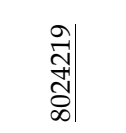 & 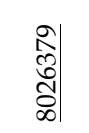 & 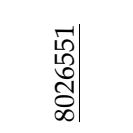 & 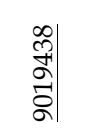 & 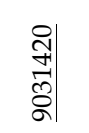 & 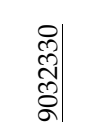 & 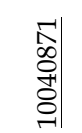 \\
\hline
\end{tabular}




\begin{tabular}{|c|c|c|c|c|c|c|c|c|c|c|}
\hline 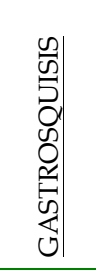 & 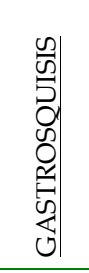 & 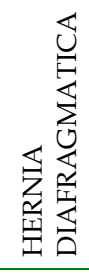 & 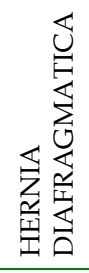 & 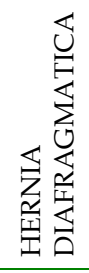 & 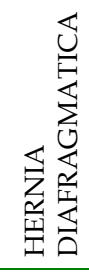 & 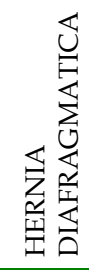 & 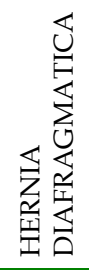 & 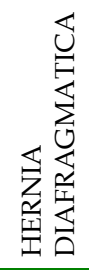 & 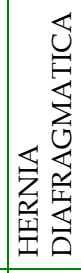 & 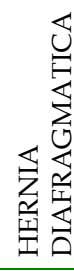 \\
\hline 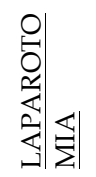 & 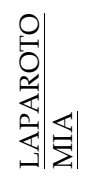 & 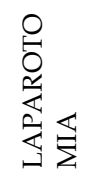 & 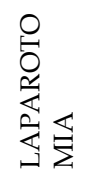 & 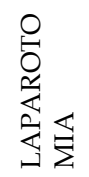 & 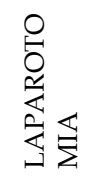 & 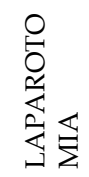 & 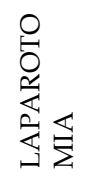 & 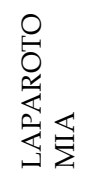 & 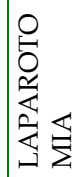 & 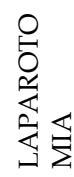 \\
\hline 艺 & $\bar{\gtrless}$ & 云 & 云 & 云 & 妾 & 文 & 云 & 妾 & ž & Zu \\
\hline ฉి & ๘) & そ) & 只 & そ & そ & 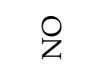 & 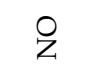 & そ & \& & ¿ \\
\hline ฉి & Oे & そ) & そ) & そ) & 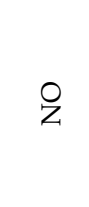 & 尺े & $\stackrel{\circ}{Z}$ & そ & \& & 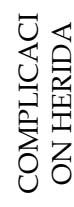 \\
\hline 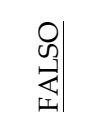 & 各 & 怘 & 怘 & 足 & 唨 & 足 & $\begin{array}{l}0 \\
\text { 思 } \\
\text { 厌 }\end{array}$ & 足 & $\begin{array}{l}\text { 蓆 } \\
\stackrel{2}{2} \approx \\
\frac{2}{>}=\end{array}$ & $\begin{array}{l}0 \\
\text { 足 } \\
\text { 压 }\end{array}$ \\
\hline$\underset{\infty}{\stackrel{0}{\mid}} \mid$ & 네 $\mid$ & 苞 & ৯ & $\begin{array}{l}\text { 足 } \\
\text { n }\end{array}$ & in & 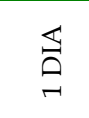 & $\underset{\neg}{\overleftrightarrow{\Delta}}$ & ৯े & F足 & $\approx \mathbb{Z}$ \\
\hline 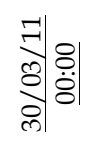 & 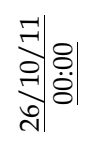 & 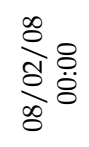 & 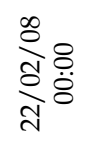 & $\begin{array}{l}\frac{\infty}{2} 8 \\
\frac{0}{2} \\
\frac{8}{0}\end{array}$ & $\begin{array}{l}\frac{\infty}{2} 8 \\
\stackrel{0}{0} 8 \\
\stackrel{0}{0}\end{array}$ & 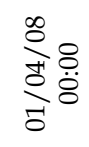 & $\begin{array}{l}\frac{\infty}{2} 8 \\
\frac{0}{0} \\
\frac{0}{0} \\
\dot{0}\end{array}$ & 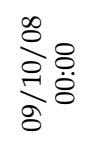 & 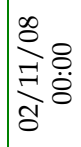 & 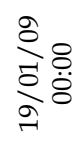 \\
\hline 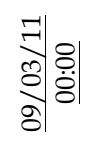 & 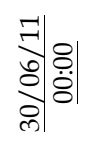 & $\begin{array}{l}\stackrel{\infty}{0}_{0}^{\infty} 8 \\
\text { مे } \\
0\end{array}$ & 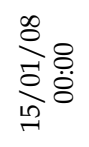 & 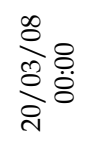 & 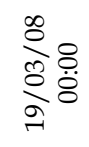 & 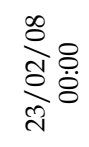 & $\begin{array}{l}\frac{\infty}{20} 8 \\
\stackrel{8}{2} \\
\frac{1}{2}\end{array}$ & 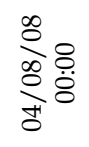 & 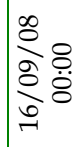 & 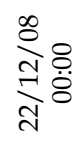 \\
\hline 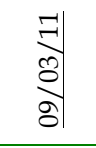 & 걀 & $\frac{\infty}{\stackrel{\infty}{0}}$ & $\frac{\infty}{\stackrel{\infty}{े}}$ & $\begin{array}{l}\stackrel{\infty}{\circ} \\
\stackrel{\text { वे }}{े}\end{array}$ & $\begin{array}{l}\stackrel{\infty}{2} \\
\stackrel{0}{\rho} \\
\text { }\end{array}$ & 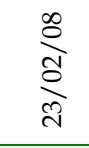 & 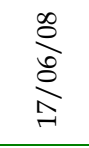 & $\begin{array}{l}\stackrel{\infty}{2} \\
\stackrel{\infty}{\infty} \\
\text { tे }\end{array}$ & $\begin{array}{l}\stackrel{\infty}{0} \\
\frac{0}{0} \\
\stackrel{\rho}{0}\end{array}$ & 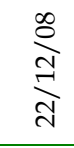 \\
\hline 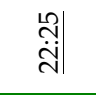 & 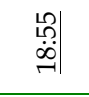 & $\stackrel{\stackrel{\vartheta}{\circ}}{\circ}$ & 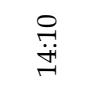 & $\begin{array}{l}\text { के } \\
\ddot{\vec{j}}\end{array}$ & مृ & $\stackrel{\stackrel{n}{\ddot{n}}}{\stackrel{\vec{\sim}}{n}}$ & 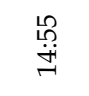 & 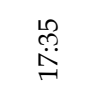 & 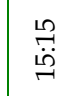 & $\begin{array}{l}\stackrel{9}{+} \\
\dot{\forall}\end{array}$ \\
\hline$\stackrel{\text { ते }}{\text { ते }}$ & $\begin{array}{c}\stackrel{0}{0} \\
\dot{0} \\
\stackrel{0}{0} \mid\end{array}$ & $\begin{array}{l}\infty \\
\stackrel{\infty}{0} \\
\dot{\infty} \\
\sim\end{array}$ & $\begin{array}{l}\stackrel{0}{h} \\
\stackrel{\leftrightarrow}{\vec{n}}\end{array}$ & 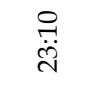 & $\begin{array}{l}\text { लै } \\
\text { हैं }\end{array}$ & 卢 & 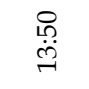 & 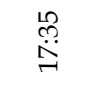 & 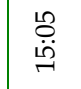 & 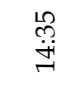 \\
\hline 党 & 유. & 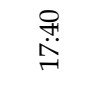 & 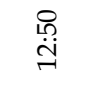 & $\stackrel{\stackrel{n}{\ddot{v}}}{ }$ & $\begin{array}{l}\text { n̊. } \\
\text { فn }\end{array}$ & 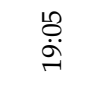 & 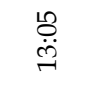 & $\stackrel{P}{\vec{\forall}}$ & $\begin{array}{l}\stackrel{P}{\ddot{H}} \\
\text { (n) }\end{array}$ & $\begin{array}{l}\stackrel{\text { In }}{\text { తn }}\end{array}$ \\
\hline | & $\begin{array}{l}\text { 号| } \\
\stackrel{\vec{n}}{\mid}\end{array}$ & 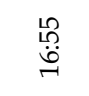 & $\stackrel{\stackrel{\llcorner}{m}}{\stackrel{\oplus}{ت}}$ & $\begin{array}{l}\stackrel{9}{4} \\
\dot{\pi}\end{array}$ & $\underset{\ddot{\ddot{\theta}}}{\stackrel{0}{\vec{r}}}$ & 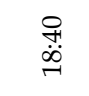 & 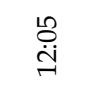 & 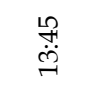 & $\stackrel{\stackrel{\vartheta}{ت}}{ت}$ & $\underset{\stackrel{\leftrightarrow}{\grave{d}}}{ }$ \\
\hline $\begin{array}{l}7= \\
\bar{a} \\
\vdots \\
8 \\
8\end{array}$ & 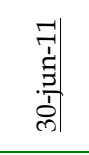 & 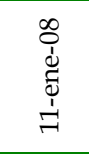 & 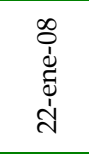 & 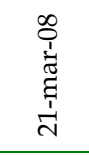 & 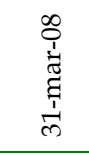 & 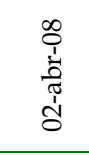 & 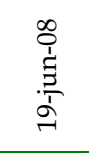 & 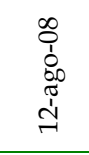 & 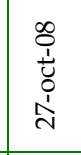 & 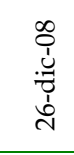 \\
\hline 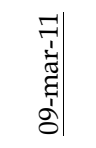 & 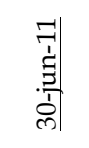 & 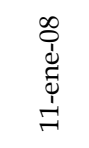 & 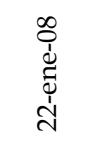 & 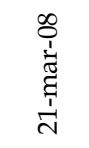 & 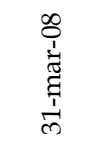 & 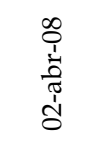 & 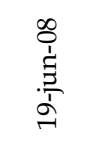 & 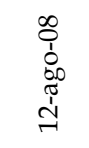 & 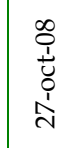 & 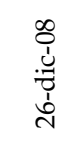 \\
\hline 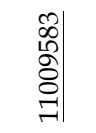 & 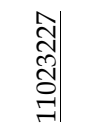 & 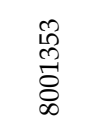 & 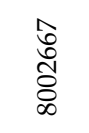 & $\begin{array}{l}\text { ठ } \\
\text { ळे } \\
\text { ठे }\end{array}$ & 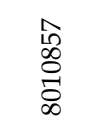 & $\begin{array}{l}8 \\
\stackrel{0}{0} \\
\vec{\Xi} \\
\stackrel{0}{0}\end{array}$ & 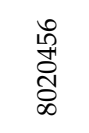 & $\begin{array}{l}\text { तิ } \\
\text { तิ } \\
\text { ర్ }\end{array}$ & $\begin{array}{l}\text { F } \\
\stackrel{1}{0} \\
\widetilde{\delta} \\
\infty\end{array}$ & 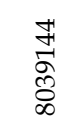 \\
\hline
\end{tabular}




\begin{tabular}{|c|c|c|c|c|c|c|c|c|c|c|}
\hline 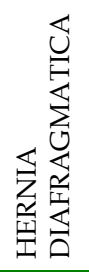 & 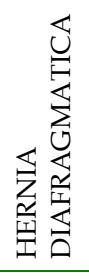 & 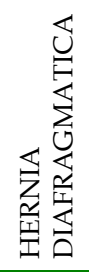 & 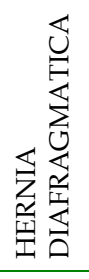 & 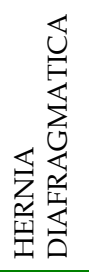 & 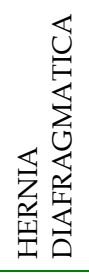 & 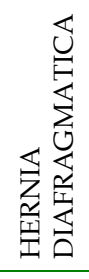 & 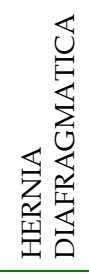 & 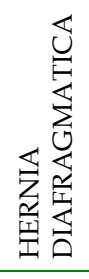 & 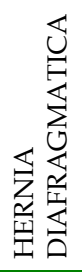 & 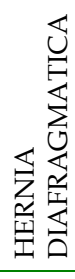 \\
\hline 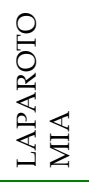 & 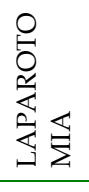 & 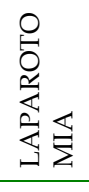 & 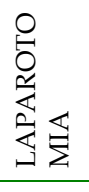 & 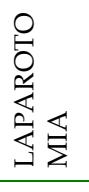 & 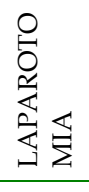 & 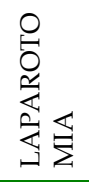 & 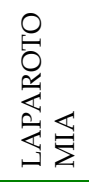 & 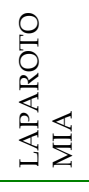 & 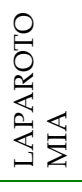 & 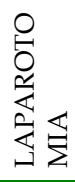 \\
\hline 文 & 文 & 云 & Z & 玄 & 文 & 交 & 文 & 交 & 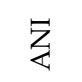 & 玄 \\
\hline そ & Z & $\stackrel{\circ}{Z}$ & $\stackrel{\circ}{Z}$ & ¿े & そ) & $\stackrel{\circ}{Z}$ & $\stackrel{\circ}{Z}$ & $\stackrel{\circ}{Z}$ & $\stackrel{\circ}{z}$ & そ \\
\hline Z & そ & $\stackrel{\circ}{ }$ & $\stackrel{\circ}{Z}$ & そे & 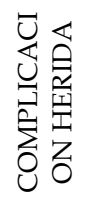 & ○े & $\stackrel{\circ}{Z}$ & そ) & そ) & Z \\
\hline 㤂 & $\begin{array}{l}\text { O } \\
\text { 崖 }\end{array}$ & 怘 & 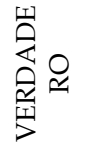 & 㤂 & 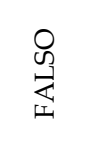 & 怘 & 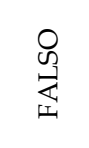 & $\begin{array}{l}\text { 呆 } \\
\text { 离 }\end{array}$ & $\begin{array}{l}\text { O } \\
\text { 崖 }\end{array}$ & 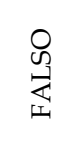 \\
\hline$\sum_{\infty}^{\infty}$ & 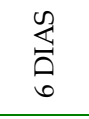 & $\sum_{0}^{\infty}$ & 员罢 & \begin{tabular}{l}
$\infty$ \\
\multirow{3}{\Delta}{} \\
$a$
\end{tabular} & 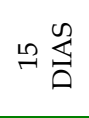 & 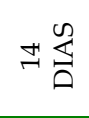 & 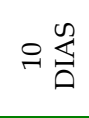 & 离 & 足 & 离 \\
\hline 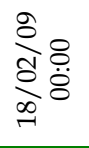 & $\frac{\frac{g}{2}}{\delta} \frac{8}{8}$ & 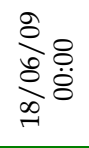 & 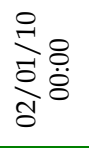 & 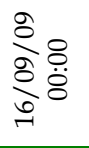 & 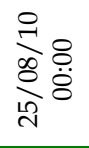 & 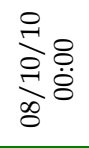 & $\sum_{\infty}^{\circ} 8$ & 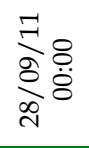 & 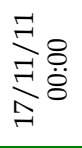 & $\underset{\nexists}{\vec{F}} 8$ \\
\hline 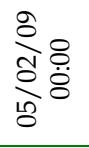 & 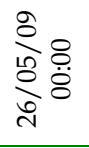 & 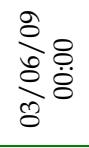 & 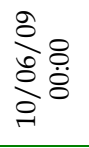 & 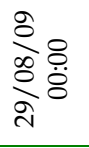 & 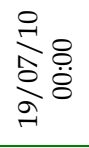 & 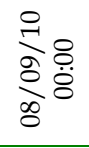 & 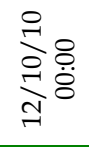 & 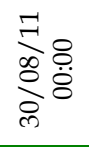 & 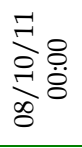 & 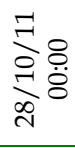 \\
\hline 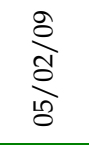 & 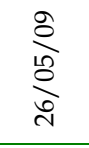 & 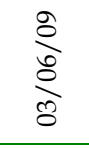 & 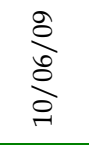 & $\begin{array}{l}\frac{\partial}{d} \\
\frac{\partial}{\partial े} \\
\text { ते }\end{array}$ & $\frac{0}{\frac{1}{5}}$ & $\frac{\stackrel{9}{0}}{\frac{0}{8}}$ & 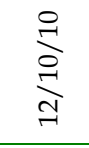 & $\begin{array}{l}\vec{F} \\
\stackrel{\infty}{\circ} \\
\stackrel{\infty}{\infty}\end{array}$ & $\underset{\infty}{\stackrel{F}{o}}$ & 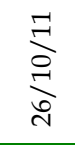 \\
\hline 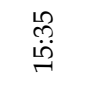 & $\begin{array}{l}\stackrel{\oplus}{m} \\
\text { ले }\end{array}$ & 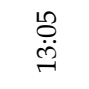 & 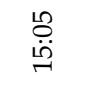 & $\begin{array}{l}\widetilde{N} \\
\stackrel{\text { ते }}{2}\end{array}$ & $\stackrel{\stackrel{\leftrightarrow}{\mathrm{U}}}{\stackrel{\mathrm{U}}{ }}$ & $\begin{array}{l}\stackrel{9}{\sharp} \\
\text { in }\end{array}$ & 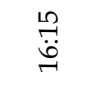 & $\stackrel{\stackrel{\varphi}{\leftrightarrow}}{\stackrel{\leftrightarrow}{\bullet}}$ & $\begin{array}{l}\stackrel{\infty}{0} \\
\stackrel{\leftrightarrow}{n}\end{array}$ & 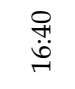 \\
\hline 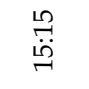 & 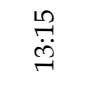 & $\underset{\stackrel{\leftrightarrow}{\leftrightarrow}}{\stackrel{8}{\sim}}$ & 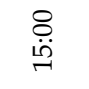 & 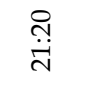 & $\stackrel{\stackrel{\leftrightarrow}{\sim}}{\underset{\sim}{ت}}$ & $\begin{array}{l}\text { तิ } \\
\text { ஸ் }\end{array}$ & 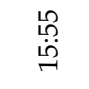 & 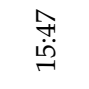 & 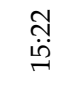 & \\
\hline 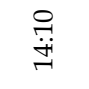 & $\stackrel{\stackrel{\Upsilon}{\ddot{ت}}}{ }$ & 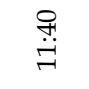 & 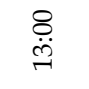 & तิ̀ & 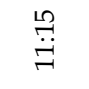 & $\begin{array}{l}\stackrel{2}{\not} \\
\ddot{n}\end{array}$ & 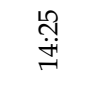 & $\begin{array}{l}\stackrel{P}{+} \\
\stackrel{H}{\sim}\end{array}$ & $\begin{array}{l}8 \\
\ddot{\theta}\end{array}$ & $\begin{array}{l}\text { గn } \\
\stackrel{\leftrightarrow}{*}\end{array}$ \\
\hline $\begin{array}{l}\stackrel{\leftrightarrow}{\pi} \\
\stackrel{\sim}{\sim}\end{array}$ & 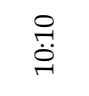 & 足 & $\begin{array}{l}\stackrel{9}{H} \\
\text { i }\end{array}$ & $\begin{array}{l}\stackrel{\infty}{\sigma} \\
\stackrel{\sigma}{\sigma}\end{array}$ & $\begin{array}{l}\stackrel{9}{+} \\
\stackrel{0}{0}\end{array}$ & 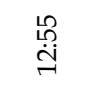 & 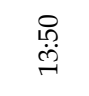 & 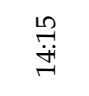 & 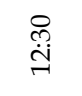 & $\begin{array}{l}\text { 표 } \\
\text { İ }\end{array}$ \\
\hline 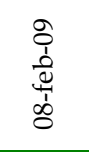 & 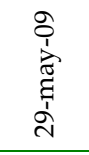 & 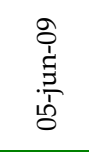 & $\begin{array}{l}0 \\
\frac{1}{1} \\
\frac{5}{1} \\
\frac{1}{0}\end{array}$ & 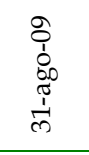 & $\begin{array}{l}0 \\
\frac{1}{3} \\
\frac{3}{10} \\
\text { i }\end{array}$ & 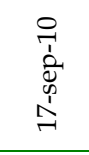 & $\begin{array}{l}0 \\
\stackrel{1}{1} \\
\stackrel{0}{0} \\
\dot{I}\end{array}$ & $\begin{array}{l}\overrightarrow{7} \\
\dot{0} \\
\stackrel{0}{0} \\
\dot{m} \\
\dot{m}\end{array}$ & 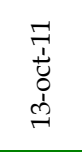 & 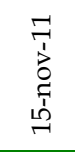 \\
\hline 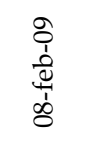 & 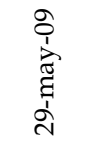 & 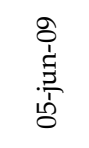 & $\begin{array}{l}0 \\
0 \\
\frac{1}{2} \\
\frac{7}{1} \\
\frac{1}{0}\end{array}$ & 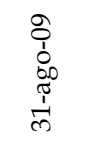 & 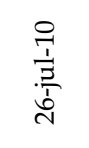 & 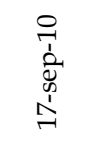 & 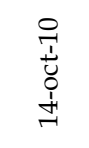 & $\begin{array}{l}\overrightarrow{7} \\
\dot{0} \\
\stackrel{0}{0} \\
\dot{1} \\
\dot{m}\end{array}$ & 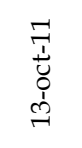 & $\begin{array}{l}\overrightarrow{7} \\
\overrightarrow{3} \\
\stackrel{5}{7} \\
\stackrel{1}{n}\end{array}$ \\
\hline $\begin{array}{l}\text { స్ } \\
\text { \&े } \\
\text { \&े }\end{array}$ & 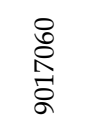 & 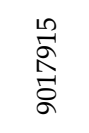 & 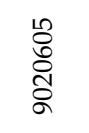 & 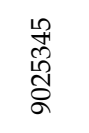 & 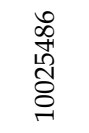 & $\begin{array}{l}\text { बे } \\
\text { oิे } \\
\text { ઠे }\end{array}$ & 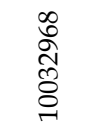 & 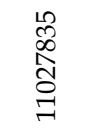 & 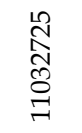 & 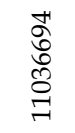 \\
\hline
\end{tabular}




\begin{tabular}{|c|c|c|c|c|c|c|c|c|}
\hline 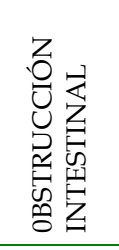 & 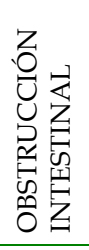 & 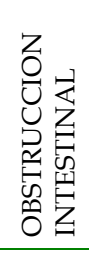 & 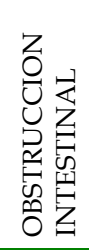 & 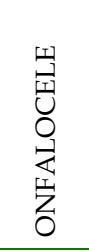 & 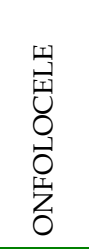 & 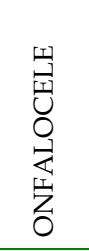 & 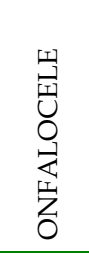 & 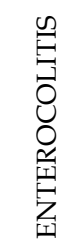 \\
\hline 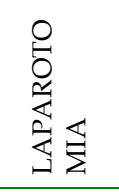 & 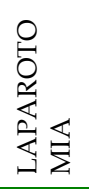 & $\begin{array}{l}0 \\
0 \\
0 \\
\frac{4}{4} \\
\text { 近 }\end{array}$ & 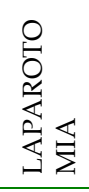 & 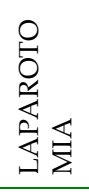 & 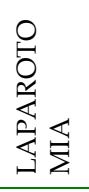 & 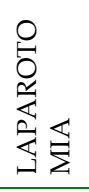 & 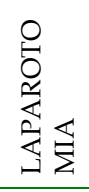 & 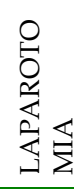 \\
\hline 玄 & 文 & 文 & 文 & 文 & 文 & 文 & 文 & 文 \\
\hline क & そ) & $\stackrel{\circ}{Z}$ & $\stackrel{\circ}{\text { Z }}$ & そ) & そ) & Z & Z & क \\
\hline 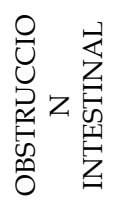 & そ) & 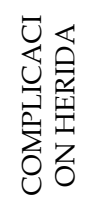 & そ) & $\stackrel{\circ}{Z}$ & $\stackrel{\circ}{Z}$ & そ) & そे & $\begin{array}{l}0 \\
\text { 崫 } \\
\text { 电 } \\
\text { \& }\end{array}$ \\
\hline 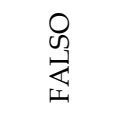 & 足 & 朶 & 盈 & 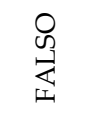 & 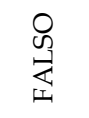 & 怘 & 务 & 蒀 \\
\hline 心总 & 놈 & $\sum_{\substack{1\\
}}^{0}$ & $\underset{\neg}{\overleftrightarrow{\Xi}}$ & $\underset{\neg}{\overleftrightarrow{\Delta}}$ & $\sum_{\substack{1\\
}}^{0}$ & $\sum_{\substack{1 \\
0}}^{0}$ & $\underset{\sim}{\mathbb{\Delta}}$ & $\underset{\sqsupset}{\overleftrightarrow{\Xi}}$ \\
\hline$\frac{\stackrel{\infty}{0}_{0}^{\infty}}{\sum_{0}^{\infty}}$ & 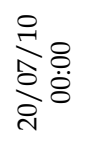 & 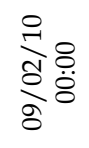 & 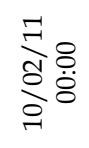 & 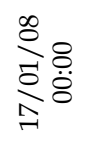 & 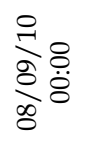 & 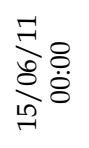 & 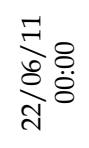 & $\frac{\sum_{0}^{\infty}}{\stackrel{\infty}{\circ}}$ \\
\hline 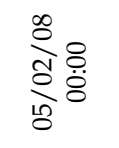 & 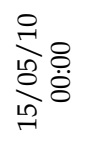 & $\begin{array}{l}\stackrel{0}{0} 8 \\
\text { 웡 }\end{array}$ & 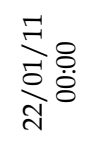 & 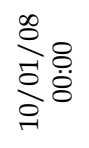 & 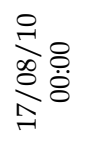 & $\begin{array}{l}\frac{F}{8} 8 \\
\frac{8}{8} \\
\frac{8}{8}\end{array}$ & 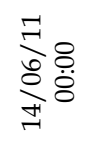 & 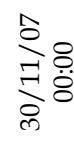 \\
\hline$\underset{\frac{1}{2}}{\stackrel{\infty}{\infty}}$ & 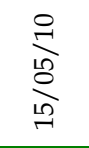 & $\begin{array}{l}\stackrel{0}{े} \\
\text { ेे }\end{array}$ & $\underset{ }{\vec{\partial}}$ & $\frac{\stackrel{\infty}{\rho}}{\stackrel{\rho}{\rho}}$ & 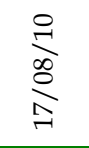 & $\frac{\overrightarrow{7}}{\frac{8}{2}}$ & 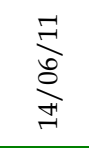 & 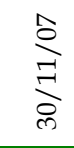 \\
\hline 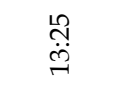 & $\begin{array}{l}\stackrel{\infty}{\ddot{H}} \\
\stackrel{\vec{H}}{\sim}\end{array}$ & $\begin{array}{l}\stackrel{P}{\ddot{H}} \\
\stackrel{n}{n}\end{array}$ & $\begin{array}{l}\stackrel{\text { }}{\mathrm{U}} \\
\text { }\end{array}$ & 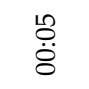 & 䒿 & $\begin{array}{l}\infty \\
\stackrel{\infty}{\sigma} \\
\stackrel{\sim}{\sigma}\end{array}$ & $\begin{array}{l}\text { 号 } \\
\text { în }\end{array}$ & 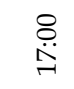 \\
\hline 菖 & $\begin{array}{l}8 \\
\text { ị } \\
\text { in }\end{array}$ & 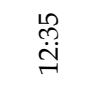 & 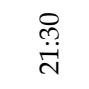 & 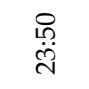 & $\begin{array}{l}\text { 另 } \\
\text { فُ }\end{array}$ & $\begin{array}{l}\stackrel{\infty}{\sigma} \\
\stackrel{\sigma}{\sigma}\end{array}$ & 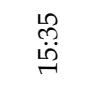 & 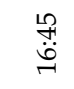 \\
\hline $\begin{array}{l}\stackrel{8}{ت} \\
\stackrel{ت}{ت}\end{array}$ & $\begin{array}{l}\stackrel{0}{0} \\
\stackrel{\leftrightarrow}{\rightarrow}\end{array}$ & $\stackrel{\stackrel{m}{\dddot{m}}}{\stackrel{m}{ت}}$ & $\begin{array}{l}\text { 요 } \\
\text { ثें }\end{array}$ & 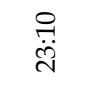 & 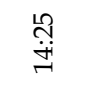 & 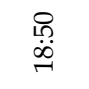 & $\begin{array}{l}\stackrel{\text { P }}{+} \\
\stackrel{+}{*}\end{array}$ & $\begin{array}{l}\stackrel{\text { ֻூ }}{\mathrm{J}} \\
\text { }\end{array}$ \\
\hline$\stackrel{\ddot{\theta}}{\ddot{\theta}}$ & 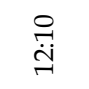 & 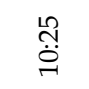 & 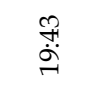 & $\begin{array}{l}\text { ते } \\
\text { }\end{array}$ & هั. & $\stackrel{\stackrel{P}{\ddot{\theta}}}{\sim}$ & 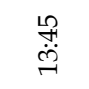 & $\stackrel{\substack{n \\
ت}}{\stackrel{ت}{ت}}$ \\
\hline 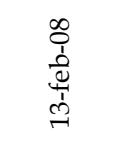 & 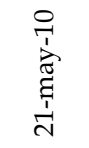 & 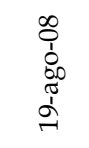 & 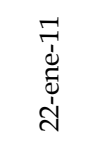 & 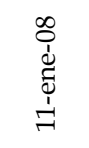 & 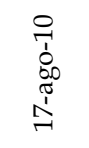 & $\begin{array}{l}\overrightarrow{7} \\
\frac{\overrightarrow{5}}{3} \\
\frac{1}{2}\end{array}$ & $\begin{array}{l}\overrightarrow{7} \\
\frac{\vec{g}}{d} \\
\frac{T}{d}\end{array}$ & $\begin{array}{l}\infty \\
\stackrel{d}{d} \\
\stackrel{\Phi}{0} \\
\stackrel{7}{7}\end{array}$ \\
\hline 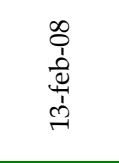 & 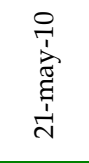 & 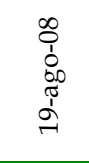 & 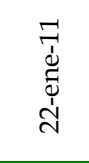 & 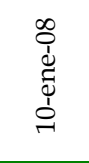 & 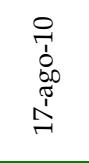 & $\begin{array}{l}\overrightarrow{7} \\
\frac{\overrightarrow{1}}{1} \\
\frac{1}{2}\end{array}$ & 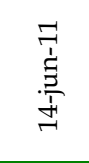 & 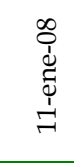 \\
\hline 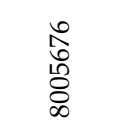 & 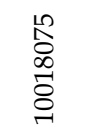 & 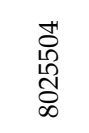 & 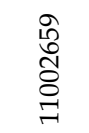 & 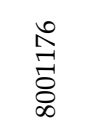 & 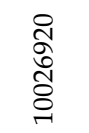 & 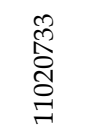 & 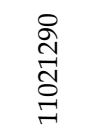 & 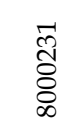 \\
\hline
\end{tabular}




\begin{tabular}{|c|c|c|c|c|c|c|c|c|c|}
\hline 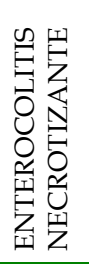 & 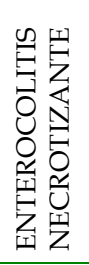 & 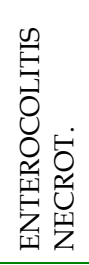 & 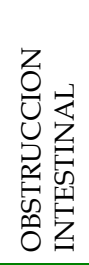 & 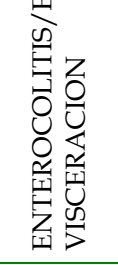 & 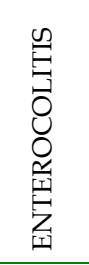 & 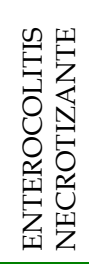 & 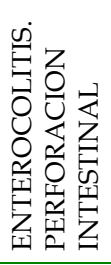 & 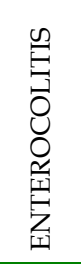 & 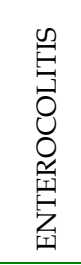 \\
\hline 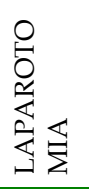 & 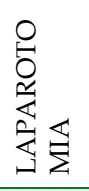 & 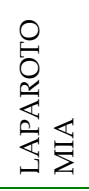 & 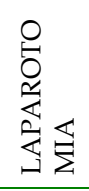 & 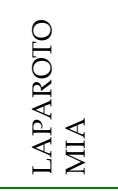 & 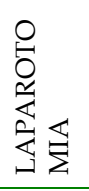 & 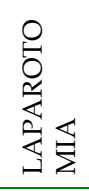 & 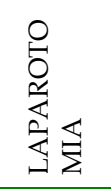 & 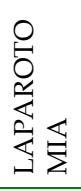 & 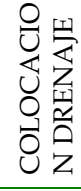 \\
\hline ż & 玄 & 菇 & 交 & ż & 文 & 文 & ż & $\begin{array}{l}z \\
\text { zy }\end{array}$ & $\begin{array}{l}z \\
\text { ż } \\
\text { o }\end{array}$ \\
\hline $\bar{\omega}$ & そ) & 只 & $\vec{\omega}$ & $\vec{w}$ & क & $\stackrel{\circ}{Z}$ & ○ & 尺 & ○ \\
\hline 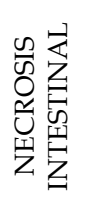 & $\stackrel{̊}{Z}$ & $\stackrel{̊}{Z}$ & 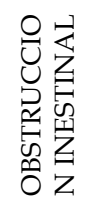 & 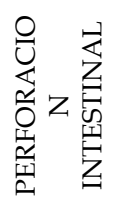 & 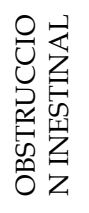 & 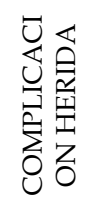 & $\stackrel{\wp}{Z}$ & 尺 & ○ \\
\hline 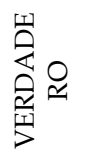 & 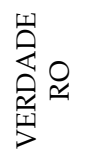 & 怘 & 怘 & 惫 & 怘 & 怘 & 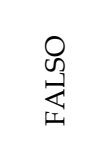 & 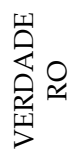 & 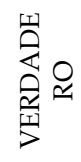 \\
\hline ¿ & 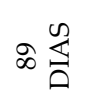 & $\Rightarrow$ & 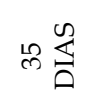 & 壮空 & 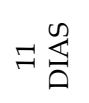 & 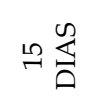 & g & $\stackrel{\infty}{\infty}$ & 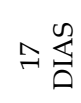 \\
\hline 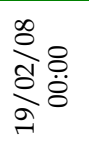 & 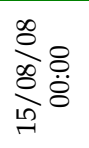 & 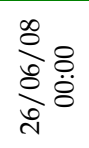 & 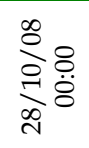 & 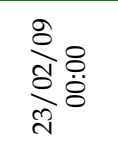 & 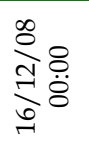 & $\begin{array}{l}\frac{\partial}{2} 8 \\
\frac{\partial}{\infty} \underset{0}{0}\end{array}$ & 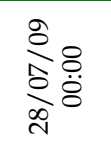 & 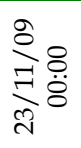 & 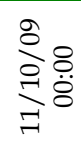 \\
\hline 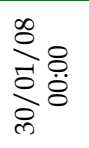 & 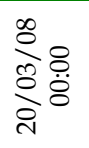 & 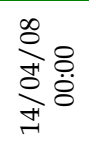 & 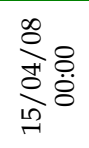 & 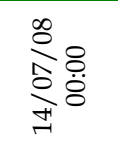 & 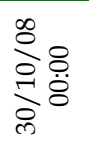 & 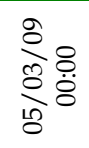 & 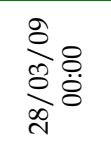 & 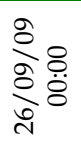 & 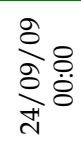 \\
\hline 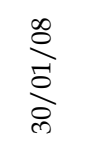 & 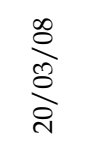 & $\frac{\stackrel{\infty}{\frac{D}{2}}}{\vec{f}}$ & $\underset{\stackrel{2}{2}}{\stackrel{\infty}{2}}$ & 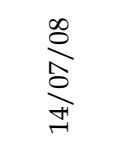 & 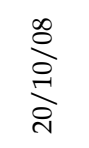 & 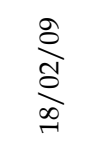 & 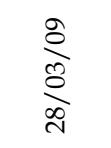 & & $\frac{\stackrel{\partial}{d}}{\partial}$ \\
\hline 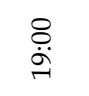 & $\stackrel{m}{\ddot{\theta}}$ & $\begin{array}{l}\stackrel{\sim}{\pi} \\
\ddot{\sim}\end{array}$ & 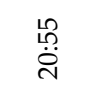 & $\stackrel{\text { ஜn }}{\stackrel{\leftrightarrow}{*}}$ & $\begin{array}{l}\ddot{\theta} \\
\ddot{8}\end{array}$ & $\begin{array}{l}\stackrel{\omega}{m} \\
\ddot{\tilde{j}}\end{array}$ & 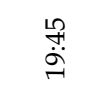 & 员 & $\begin{array}{l}\stackrel{9}{+!} \\
\stackrel{-1}{-}\end{array}$ \\
\hline $\begin{array}{l}\stackrel{0}{h} \\
\stackrel{0}{\oplus}\end{array}$ & 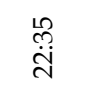 & 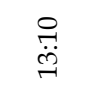 & 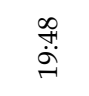 & $\begin{array}{l}\stackrel{8}{+} \\
\stackrel{+}{\sim}\end{array}$ & $\begin{array}{l}\stackrel{\leftrightarrow}{.} \\
\ddot{8}\end{array}$ & 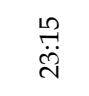 & $\begin{array}{l}\text { 욤 } \\
\stackrel{9}{\circ}\end{array}$ & 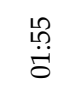 & $\begin{array}{l}\widetilde{\curvearrowright} \\
\stackrel{\sim}{-}\end{array}$ \\
\hline 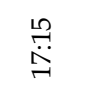 & $\underset{\ddot{\sharp}}{\stackrel{\text { }}{ }}$ & $\begin{array}{l}\text { ஸे } \\
\stackrel{\text { I}}{ }\end{array}$ & $\begin{array}{l}\stackrel{9}{+!} \\
\stackrel{-1}{-}\end{array}$ & 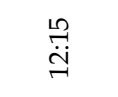 & 号 & $\begin{array}{l}\stackrel{\circ}{\ddot{~}} \\
\ddot{\text { an }}\end{array}$ & 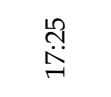 & $\begin{array}{l}\stackrel{\leftrightarrow}{\ddot{g}} \\
\stackrel{\sigma}{\sigma}\end{array}$ & $\begin{array}{l}\text { ָิ } \\
\stackrel{-}{n}\end{array}$ \\
\hline 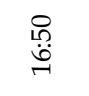 & $\begin{array}{l}\text { ले } \\
\stackrel{\tilde{n}}{2}\end{array}$ & 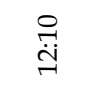 & 号 & 早 & 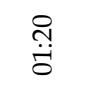 & 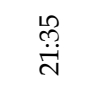 & $\begin{array}{l}\stackrel{8}{\circ} \\
\stackrel{0}{0}\end{array}$ & ণิ̊̄ & $\begin{array}{l}\text { 早 } \\
\text { : }\end{array}$ \\
\hline 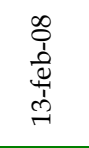 & \begin{tabular}{l}
$\infty$ \\
0 \\
1 \\
$\frac{1}{0}$ \\
\multicolumn{1}{l}{} \\
0
\end{tabular} & 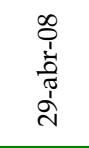 & 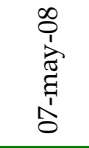 & $\begin{array}{l}\infty \\
0 \\
0 \\
0 \\
0 \\
0 \\
0 \\
0\end{array}$ & $\begin{array}{l}\infty \\
0 \\
1 \\
0 \\
0 \\
0 \\
0 \\
0\end{array}$ & 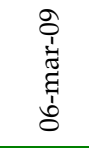 & 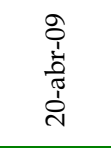 & 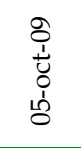 & 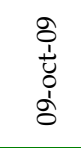 \\
\hline 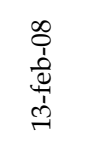 & 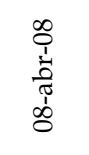 & \begin{tabular}{l}
$\infty$ \\
0 \\
$\frac{1}{0}$ \\
\multicolumn{1}{|c}{} \\
ते
\end{tabular} & 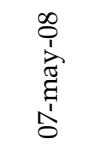 & $\begin{array}{l}\infty \\
0 \\
\vdots \\
0 \\
0 \\
0 \\
0\end{array}$ & $\begin{array}{l}\infty \\
0 \\
1 \\
0 \\
0 \\
0 \\
0\end{array}$ & 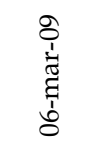 & 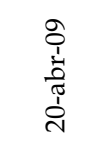 & 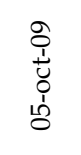 & 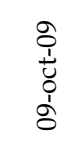 \\
\hline $\begin{array}{l}\infty \\
\infty \\
\stackrel{0}{0} \\
\stackrel{0}{\infty} \\
\infty\end{array}$ & $\begin{array}{l}\infty \\
\stackrel{ి}{0} \\
\stackrel{\Xi}{\infty}\end{array}$ & $\begin{array}{l}\stackrel{0}{0} \\
\text { J } \\
\stackrel{0}{0}\end{array}$ & $\begin{array}{l}\stackrel{0}{0} \\
\stackrel{0}{0} \\
\stackrel{1}{1} \\
\infty\end{array}$ & 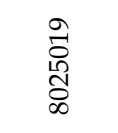 & 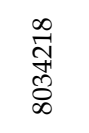 & 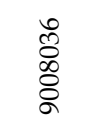 & 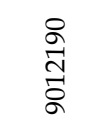 & $\begin{array}{l}\text { ठ } \\
\text { তิ } \\
\text { ઠे }\end{array}$ & $\begin{array}{l}\text { ते } \\
\text { ลे } \\
\text { ळे }\end{array}$ \\
\hline
\end{tabular}




\begin{tabular}{|c|c|c|c|c|c|c|c|c|c|}
\hline 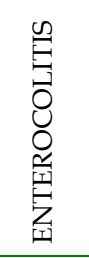 & 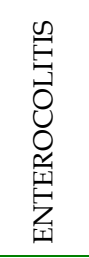 & 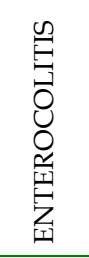 & 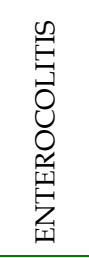 & 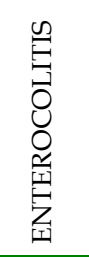 & 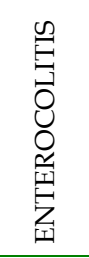 & 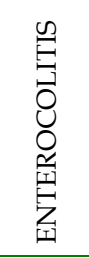 & 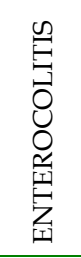 & 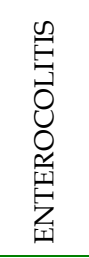 & 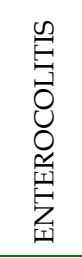 \\
\hline 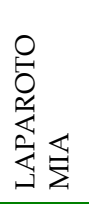 & 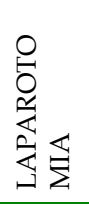 & 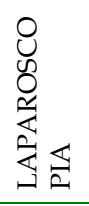 & 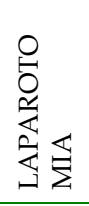 & 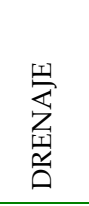 & 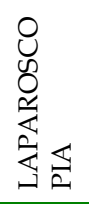 & 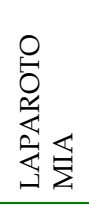 & 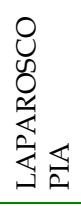 & 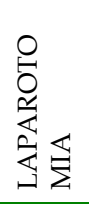 & 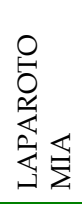 \\
\hline Z⿱ Z⿱㇒士 & 云 & 文 & 文 & Z Z & 文 & 文 & 文 & 文 & Zu \\
\hline $\bar{\omega}$ & $\bar{\omega}$ & Z & w & 尺 & w & w & $\stackrel{\circ}{Z}$ & そ) & क \\
\hline 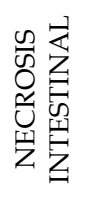 & 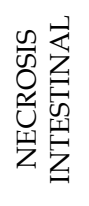 & $\stackrel{̊}{z}$ & 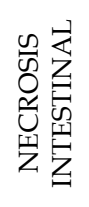 & 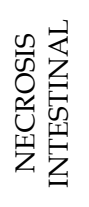 & 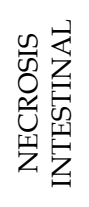 & 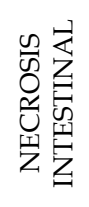 & 尺 & 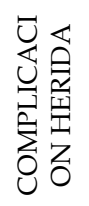 & 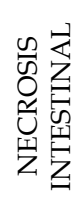 \\
\hline 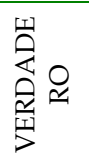 & 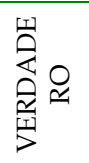 & 足 & 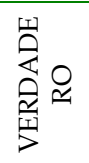 & 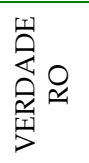 & 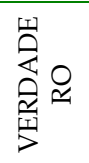 & 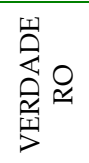 & 怘 & 足 & 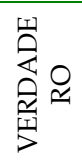 \\
\hline 萑号 & $\underset{\infty}{\infty}$ & i & $F \stackrel{?}{\Delta}$ & $\underset{\Lambda}{\mathbb{S}}$ & $\sigma \sum_{0}^{0}$ & 禹 & 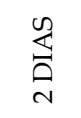 & के & $\infty \sum^{\infty}$ \\
\hline 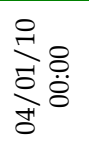 & 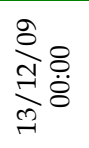 & 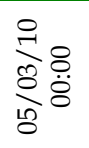 & 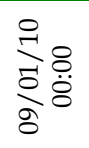 & 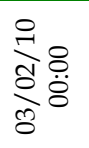 & 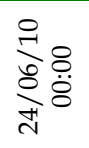 & 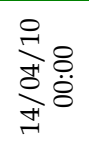 & 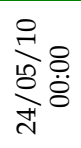 & 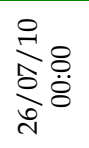 & 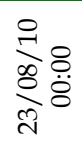 \\
\hline 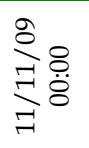 & $\begin{array}{l}\stackrel{2}{2} 8 \\
\text { 잉 } \\
\stackrel{0}{0}\end{array}$ & 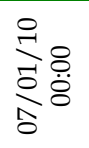 & 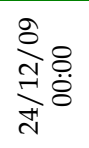 & 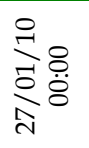 & 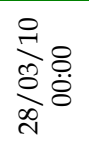 & 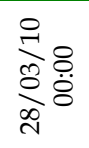 & 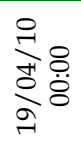 & 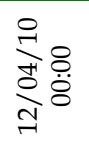 & 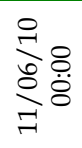 \\
\hline$\underset{\exists}{\stackrel{8}{F}}$ & 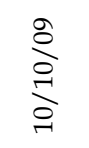 & 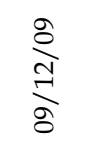 & 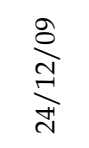 & 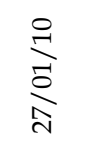 & $\underset{\substack{\infty \\
\stackrel{\infty}{\infty}}}{\stackrel{0}{\infty}}$ & $\underset{\substack{\infty \\
\stackrel{\infty}{\infty}}}{\stackrel{0}{\infty}}$ & $\frac{\stackrel{2}{\frac{1}{2}}}{\frac{\partial}{g}}$ & 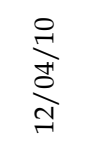 & $\frac{\stackrel{9}{8}}{\stackrel{8}{7}}$ \\
\hline $\begin{array}{l}\stackrel{0}{0} \\
\ddot{\tilde{\nu}}\end{array}$ & $\stackrel{\stackrel{ }{ت}}{\stackrel{ت}{ت}}$ & $\begin{array}{l}\stackrel{\leftrightarrow}{\leftrightarrow} \\
\stackrel{\leftrightarrow}{\delta}\end{array}$ & $\begin{array}{l}\text { ํ․ } \\
\text { ஸे }\end{array}$ & ڤ̊n. & 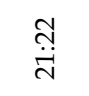 & 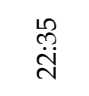 & 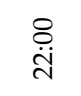 & 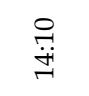 & $\begin{array}{l}\stackrel{\oplus}{\text { ते }} \\
\dot{\vec{n}}\end{array}$ \\
\hline $\begin{array}{l}\stackrel{\oplus}{.} \\
\text { ते }\end{array}$ & 号 & $\begin{array}{l}\stackrel{\leftrightarrow}{\leftrightarrow} \\
\stackrel{\delta}{\delta}\end{array}$ & ণิ ণิ & ֶֶֶ. & 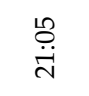 & $\stackrel{\stackrel{\leftrightarrow}{\sharp}}{\ddot{\sim}}$ & $\begin{array}{l}\stackrel{P}{H} \\
\dot{\vec{n}}\end{array}$ & $\begin{array}{l}\stackrel{8}{\leftrightarrow} \\
\stackrel{+}{+}\end{array}$ & $\begin{array}{l}\tilde{N} \\
\dot{\sim}\end{array}$ \\
\hline $\begin{array}{l}\text { 谓 } \\
\text { In }\end{array}$ & 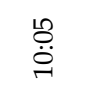 & $\begin{array}{l}\stackrel{\circ}{\dot{b}} \\
\text { }\end{array}$ & $\begin{array}{l}\stackrel{\circ}{\circ} \\
\stackrel{\alpha}{2}\end{array}$ & $\begin{array}{l}\text { ? } \\
\stackrel{4}{4}\end{array}$ & 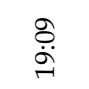 & 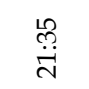 & $\begin{array}{l}\text { Фे. } \\
\text { ते }\end{array}$ & $\begin{array}{l}\text { ்ָ } \\
\text { }\end{array}$ & $\begin{array}{l}\stackrel{n}{\ddot{a}} \\
\stackrel{a}{a}\end{array}$ \\
\hline $\begin{array}{l}\stackrel{+}{4} \\
\dot{i}\end{array}$ & $\begin{array}{l}\stackrel{10}{\ddot{\theta}} \\
\ddot{\ddot{\theta}}\end{array}$ & $\begin{array}{l}\stackrel{0}{0} \\
\ddot{8}\end{array}$ & 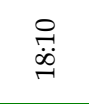 & $\begin{array}{l}\stackrel{\leftrightarrow}{0} \\
\stackrel{\tilde{N}}{ }\end{array}$ & $\begin{array}{l}\stackrel{\infty}{\infty} \\
\stackrel{\infty}{\sim}\end{array}$ & $\begin{array}{l}\stackrel{\infty}{\infty} \\
\stackrel{\infty}{\sim}\end{array}$ & $\begin{array}{l}\stackrel{0}{h} \\
\dot{\infty} \\
\stackrel{\infty}{\sim}\end{array}$ & $\underset{\stackrel{\leftrightarrow}{\ominus}}{\stackrel{\circ}{ت}}$ & $\begin{array}{c}\stackrel{0}{\infty} \\
\dot{\infty} \\
\stackrel{\infty}{\sim}\end{array}$ \\
\hline 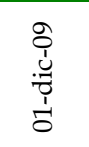 & 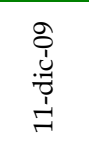 & 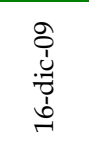 & 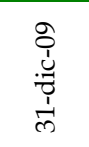 & 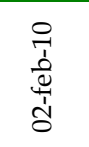 & 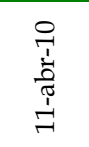 & 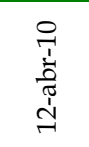 & $\begin{array}{l}0 \\
\stackrel{1}{1} \\
\frac{0}{0} \\
\stackrel{1}{1} \\
\text { N. }\end{array}$ & 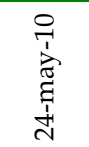 & 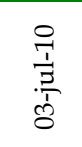 \\
\hline 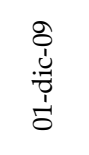 & 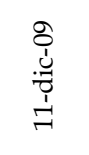 & 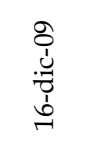 & 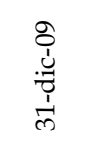 & 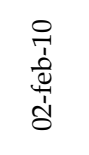 & 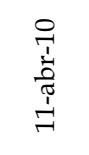 & 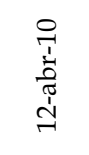 & 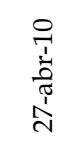 & 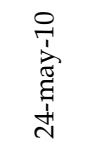 & $\begin{array}{l}0 \\
\frac{1}{3} \\
\frac{5}{3} \\
\frac{1}{8}\end{array}$ \\
\hline 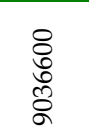 & 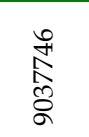 & $\begin{array}{l}\bar{్} \\
\infty \\
\infty \\
\delta ু\end{array}$ & 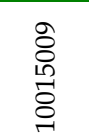 & 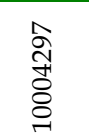 & 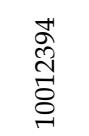 & 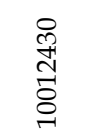 & 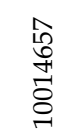 & 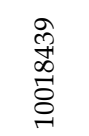 & 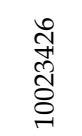 \\
\hline
\end{tabular}




\begin{tabular}{|c|c|c|c|c|c|c|c|c|c|c|c|}
\hline 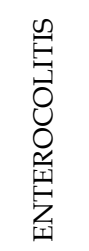 & 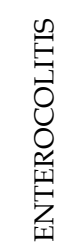 & 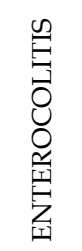 & 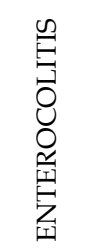 & 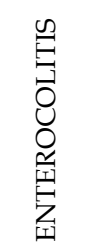 & 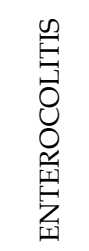 & 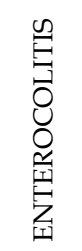 & 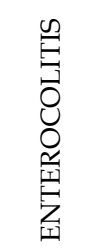 & 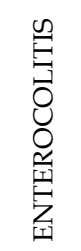 & 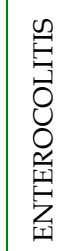 & 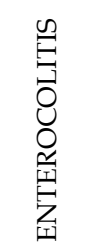 & 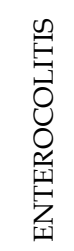 \\
\hline 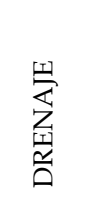 & 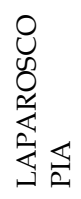 & 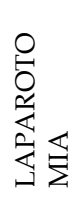 & 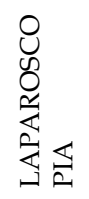 & 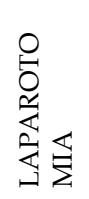 & 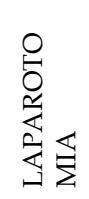 & 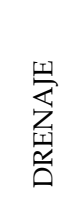 & 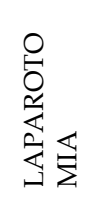 & 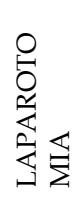 & 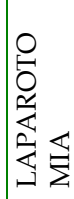 & 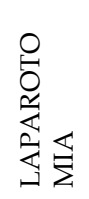 & 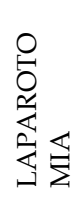 \\
\hline $\begin{array}{l}Z \\
\text { Z } \\
\text { D }\end{array}$ & 文 & 文 & 文 & 云 & $\underset{<}{Z}$ & Z & 云 & 云 & $\underset{4}{Z}$ & $\underset{4}{\mathrm{Z}}$ & 文 \\
\hline そ) & そ) & के & Z & क & क & $\stackrel{O}{Z}$ & ळ & そ & क & क & $\bar{\omega}$ \\
\hline 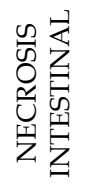 & そ) & 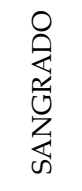 & $\stackrel{\vartheta}{Z}$ & Z & 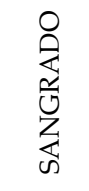 & 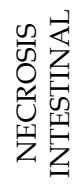 & 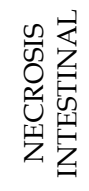 & 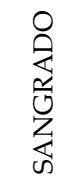 & $\stackrel{\rho}{Z}$ & $\stackrel{\circ}{Z}$ & 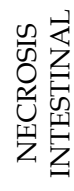 \\
\hline 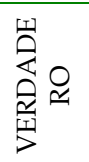 & 菖 & 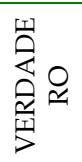 & 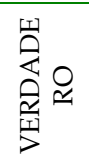 & 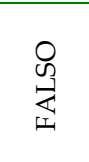 & 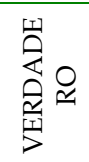 & 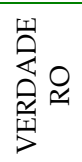 & 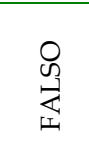 & 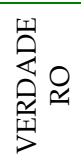 & 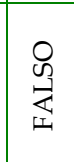 & 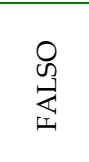 & 足 \\
\hline$\underset{\neg}{\overleftrightarrow{D}}$ & $\underset{\Omega}{\infty}$ & $\underset{\Xi}{\overparen{\Xi}}$ & 怘导 & m这 & 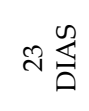 & $\rightarrow \sum_{0}^{\infty}$ & ڤ艹 & $\underset{\sim}{\overleftrightarrow{\theta}}$ & 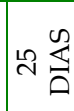 & ה & ণ \\
\hline$\frac{0}{\sum_{2}^{2}} \frac{8}{8}$ & 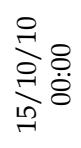 & 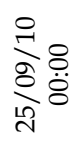 & 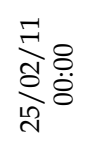 & 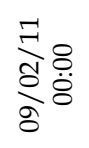 & 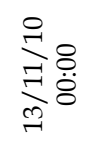 & 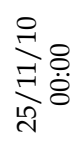 & 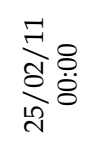 & 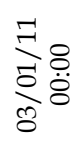 & 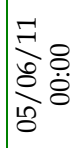 & 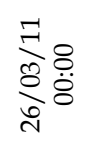 & $\begin{array}{l}\underset{8}{0} 8 \\
\underset{d}{\infty} \stackrel{0}{0}\end{array}$ \\
\hline 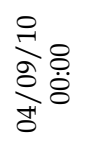 & 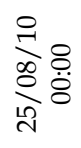 & 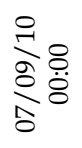 & 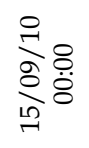 & 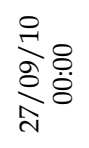 & 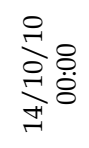 & 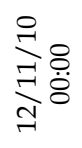 & 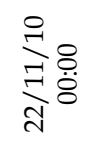 & 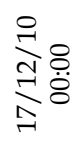 & 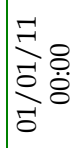 & 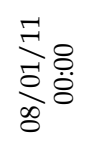 & $\begin{array}{l}\frac{F}{8} 8 \\
\frac{8}{8} \\
\frac{8}{8}\end{array}$ \\
\hline $\begin{array}{l}\stackrel{0}{0} \\
\stackrel{\infty}{0} \\
\stackrel{\rho}{\sigma}\end{array}$ & $\underset{\substack{0 \\
\stackrel{\infty}{2}}}{\stackrel{0}{\sim}}$ & $\frac{\stackrel{0}{2}}{\frac{2}{2}}$ & $\frac{0}{2}$ & 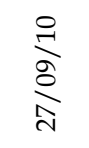 & 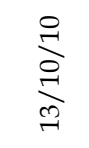 & 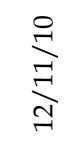 & 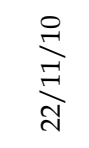 & $\underset{ }{\stackrel{0}{I}}$ & 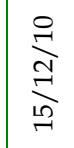 & $\underset{\infty}{\vec{\delta}}$ & $\frac{\vec{\delta}}{\delta}$ \\
\hline 品 & 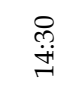 & 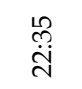 & त्ञ & $\begin{array}{l}\text { 유. } \\
\text { in }\end{array}$ & 员 & $\begin{array}{l}\stackrel{0}{0} \\
\stackrel{0}{8}\end{array}$ & 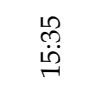 & 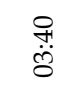 & $\stackrel{\stackrel{n}{\overparen{H}}}{\stackrel{\ddot{H}}{\sim}}$ & $\begin{array}{l}\infty \\
\stackrel{\infty}{\tilde{n}} \\
\stackrel{\tilde{N}}{2}\end{array}$ & $\begin{array}{l}\stackrel{\infty}{\sharp} \\
\text { đ̇ }\end{array}$ \\
\hline 哭 & $\stackrel{P}{\stackrel{\forall}{\forall}}$ & 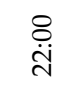 & $\stackrel{\ddot{\theta}}{\ddot{\theta}}$ & 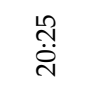 & 泀 & $\begin{array}{l}\stackrel{0}{0} \\
\stackrel{0}{8}\end{array}$ & ิㅠㅁ & $\begin{array}{l}\text { भ } \\
\text { ஸे }\end{array}$ & 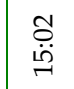 & $\begin{array}{l}\text { f̣ } \\
\text { ते }\end{array}$ & $\begin{array}{l}\stackrel{ }{\sharp} \\
\text { ה̀ }\end{array}$ \\
\hline 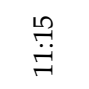 & 号 & 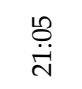 & $\stackrel{\text { ते }}{\text { in }}$ & $\begin{array}{l}0 \\
\substack{\dot{\phi} \\
\sim}\end{array}$ & 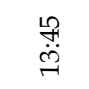 & $\begin{array}{l}\text { 요 } \\
\ddot{8}\end{array}$ & $\stackrel{8}{\stackrel{\leftrightarrow}{+}}$ & 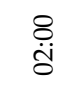 & 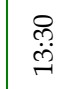 & $\begin{array}{l}\stackrel{0}{\infty} \\
\ddot{\infty}\end{array}$ & 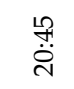 \\
\hline 号 & 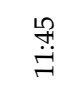 & 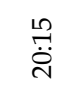 & 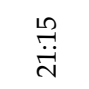 & 虽 & 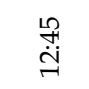 & $\begin{array}{l}\stackrel{\circ}{\circ} \\
\stackrel{8}{8}\end{array}$ & 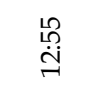 & 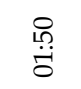 & 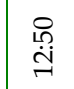 & 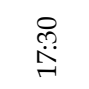 & 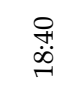 \\
\hline 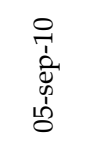 & 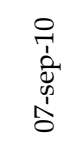 & 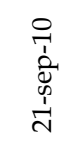 & 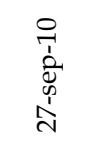 & 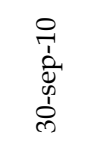 & 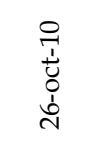 & 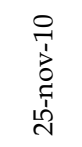 & 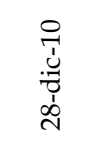 & 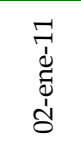 & 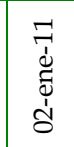 & 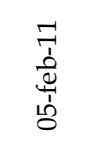 & 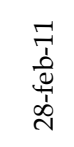 \\
\hline 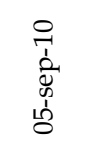 & 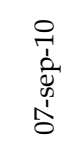 & 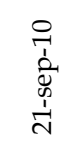 & 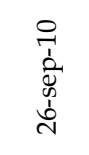 & 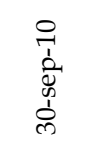 & 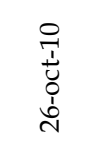 & 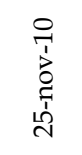 & 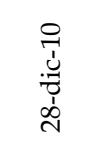 & 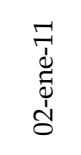 & 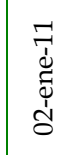 & 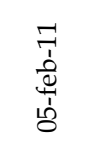 & 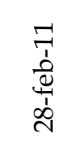 \\
\hline 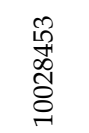 & 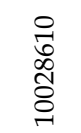 & 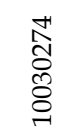 & 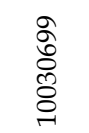 & 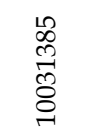 & 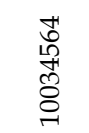 & 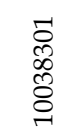 & 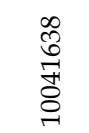 & 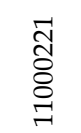 & 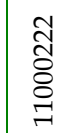 & $\begin{array}{l}0 \\
\stackrel{0}{0} \\
\text { †ै } \\
\stackrel{0}{7}\end{array}$ & $\begin{array}{l}\text { م } \\
\stackrel{1}{\hat{~}} \\
\stackrel{0}{\sigma}\end{array}$ \\
\hline
\end{tabular}




\begin{tabular}{|c|c|c|c|c|c|c|c|c|c|}
\hline 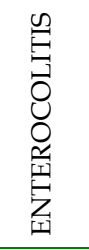 & 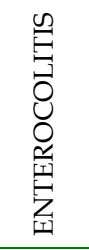 & 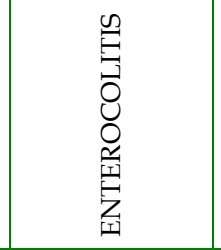 & 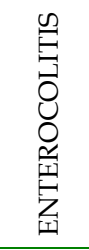 & 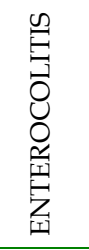 & 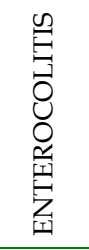 & 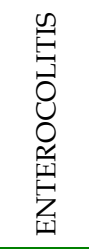 & 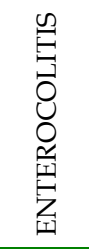 & 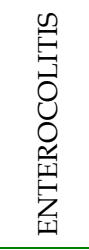 & 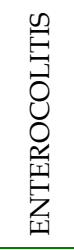 \\
\hline 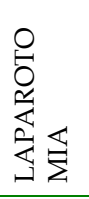 & 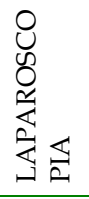 & 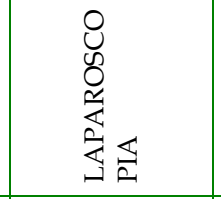 & 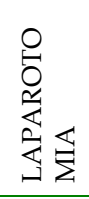 & 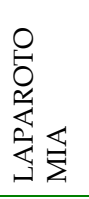 & 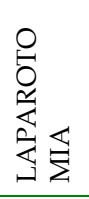 & 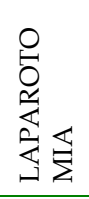 & 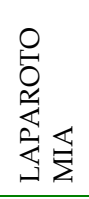 & 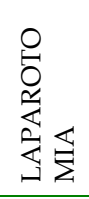 & 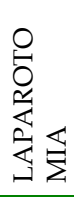 \\
\hline ż & Z & ż & Zট̆ & Z & ż & Z⿱ Z⿱㇒冋 & $\begin{array}{l}z \\
\text { z } \\
\text { D }\end{array}$ & Z & $\sum_{4}$ \\
\hline क & $\varpi$ & $\vec{\omega}$ & $\bar{\omega}$ & ๘ & $\bar{\omega}$ & $\stackrel{\circ}{Z}$ & $\stackrel{\circ}{Z}$ & そ) & 尺 \\
\hline 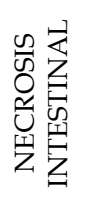 & $\stackrel{\circ}{Z}$ & 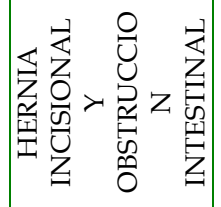 & 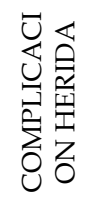 & 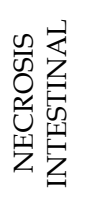 & 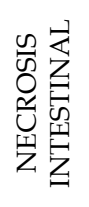 & ○ & $\stackrel{\circ}{Z}$ & そ) & 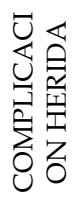 \\
\hline 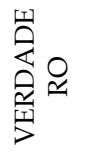 & 怘 & 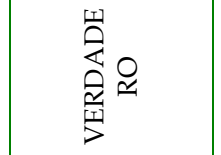 & 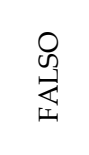 & 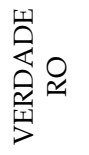 & 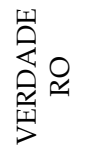 & 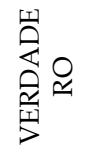 & 惫 & 艿 & 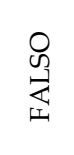 \\
\hline 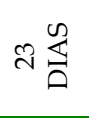 & m & 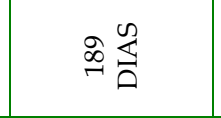 & 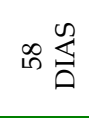 & $\because \underset{Z}{0}$ & $\sum_{\infty}^{\infty}$ & भु这 & ल & 的景 & 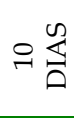 \\
\hline 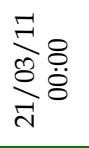 & $\begin{array}{l}\vec{F} 8 \\
\frac{\infty}{8} \stackrel{8}{0} \\
\dot{0}\end{array}$ & $\begin{array}{l}\vec{F} 8 \\
\stackrel{\overrightarrow{7}}{\stackrel{9}{8}}\end{array}$ & 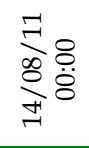 & $\begin{array}{l}\text { Fo } \\
\text { so } \\
\frac{0}{8}\end{array}$ & 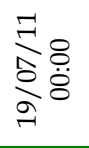 & $\begin{array}{l}71 \\
\frac{5}{20} \\
\frac{0}{8}\end{array}$ & 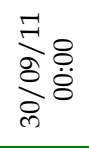 & 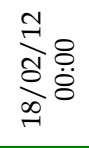 & 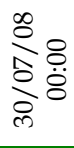 \\
\hline 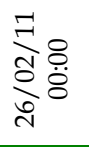 & 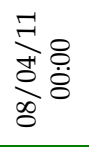 & 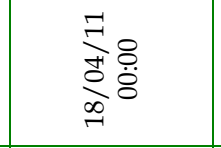 & 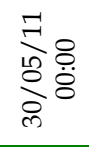 & 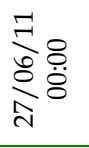 & 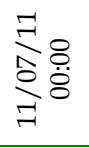 & 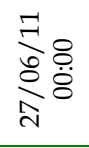 & $\begin{array}{l}\text { Fo } \\
\frac{0}{8} 8 \\
0\end{array}$ & $\sum_{i}^{\vec{F}} 8$ & 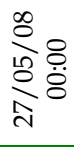 \\
\hline 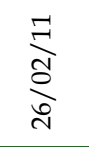 & $\underset{\infty}{\stackrel{F}{\infty}}$ & 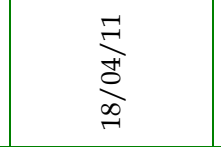 & 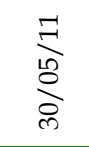 & 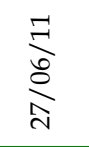 & $\underset{ }{\stackrel{F}{E}}$ & 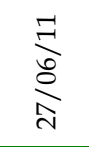 & $\frac{\sqrt{5}}{\frac{5}{8}}$ & $\underset{\text { I }}{\stackrel{\Xi}{E}}$ & 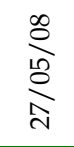 \\
\hline$\stackrel{\circ}{\ddot{g}}$ & $\begin{array}{l}\stackrel{10}{\ddot{B}} \\
\ddot{8}\end{array}$ & $\stackrel{\stackrel{\vartheta}{\ddot{\sigma}}}{ }$ & 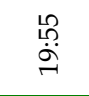 & $\underset{\stackrel{\leftrightarrow}{+}}{\stackrel{\leftrightarrow}{+}}$ & 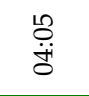 & 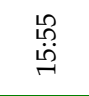 & $\begin{array}{l}\stackrel{\infty}{\circ} \\
\dot{\sigma}\end{array}$ & 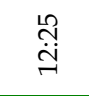 & 员 \\
\hline 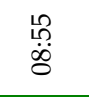 & $\begin{array}{l}\stackrel{L}{0} \\
\ddot{8}\end{array}$ & 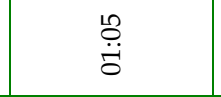 & $\begin{array}{l}\stackrel{0}{\leftrightarrow} \\
\stackrel{\leftrightarrow}{\sigma}\end{array}$ & $\begin{array}{l}\stackrel{0}{\leftrightarrow} \\
\stackrel{\leftrightarrow}{\leftrightarrow}\end{array}$ & 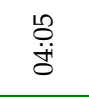 & $\begin{array}{l}\text { 尔 } \\
\text { in }\end{array}$ & $\begin{array}{l}\stackrel{\leftrightarrow}{\dot{\sigma}} \\
\stackrel{\sigma}{\sigma}\end{array}$ & $\stackrel{\stackrel{L}{ت}}{\stackrel{\Xi}{ت}}$ & 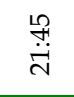 \\
\hline $\begin{array}{l}\stackrel{8}{0} \\
\dot{\phi}\end{array}$ & 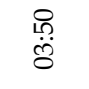 & $\stackrel{\circ}{\ddot{8}}$ & 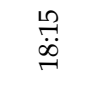 & 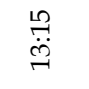 & $\begin{array}{l}\tilde{O} \\
\ddot{\delta}\end{array}$ & 胥 & तิ & 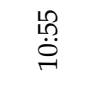 & $\begin{array}{l}\text { I্ } \\
\stackrel{n}{0}\end{array}$ \\
\hline $\begin{array}{l}\stackrel{8}{0} \\
\text { ஸे }\end{array}$ & $\begin{array}{l}\stackrel{10}{\ddot{g}} \\
\ddot{8}\end{array}$ & 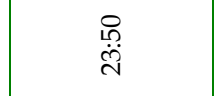 & 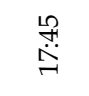 & 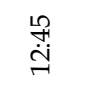 & $\begin{array}{l}\stackrel{\leftrightarrow}{0} \\
\ddot{\delta}\end{array}$ & 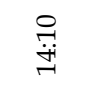 & $\begin{array}{l}\stackrel{\leftrightarrow}{\underline{n}} \\
\text { ते }\end{array}$ & $\stackrel{8}{\circ}$ & 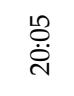 \\
\hline 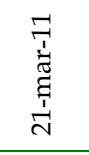 & 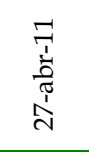 & 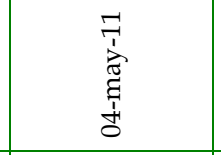 & 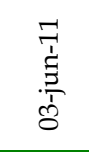 & $\begin{array}{l}7 \\
\frac{7}{3} \\
\frac{7}{8} \\
8\end{array}$ & 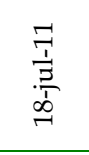 & $\begin{array}{l}\frac{7}{7} \\
\frac{3}{9} \\
\frac{4}{4}\end{array}$ & 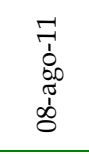 & 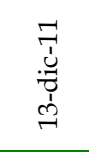 & 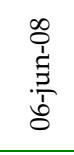 \\
\hline 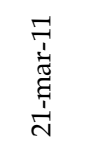 & 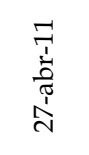 & 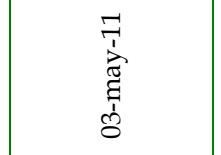 & $\begin{array}{l}\overrightarrow{7} \\
\frac{\overrightarrow{1}}{5} \\
\frac{\sqrt{6}}{\delta}\end{array}$ & $\begin{array}{l}\frac{7}{7} \\
\frac{7}{1} \\
8 \\
8\end{array}$ & 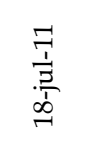 & $\begin{array}{l}\overrightarrow{7} \\
\frac{\overrightarrow{7}}{\overrightarrow{7}} \\
\frac{\lambda}{\lambda}\end{array}$ & 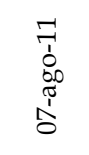 & 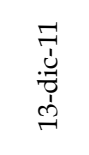 & $\begin{array}{l}\infty \\
0 \\
\vdots \\
\vdots \\
\frac{1}{1} \\
0 \\
0\end{array}$ \\
\hline 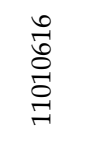 & $\begin{array}{l}\vec{\infty} \\
o \\
\stackrel{+}{0} \\
\overrightarrow{0} \\
\overrightarrow{7}\end{array}$ & $\begin{array}{l}\stackrel{0}{0} \\
10 \\
0 \\
0 \\
0 \\
7\end{array}$ & $\begin{array}{l}\vec{\sigma} \\
\text { बे } \\
\stackrel{5}{\sigma}\end{array}$ & 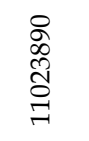 & 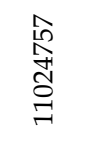 & 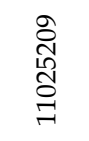 & 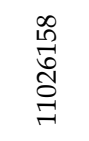 & 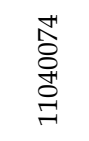 & 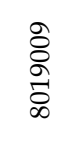 \\
\hline
\end{tabular}

